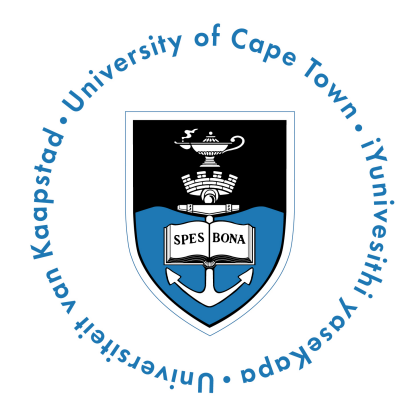

\title{
Development of a Kinetic Model for Low Temperature Fischer-Tropsch Synthesis
}

\author{
Imaad Davies \\ B. Sc. Chemical Engineering (Honours) \\ Department of Chemical Engineering \\ University of Cape Town
}

Dissertation submitted in fulfilment of the degree of Master of Science in Chemical Engineering October 2020 
The copyright of this thesis vests in the author. No quotation from it or information derived from it is to be published without full acknowledgement of the source. The thesis is to be used for private study or noncommercial research purposes only.

Published by the University of Cape Town (UCT) in terms of the non-exclusive license granted to UCT by the author. 



\section{Abstract}

Globally, there is a need to replace our dependence on fossil fuels as the main source of energy. This requires a shift towards renewable and sustainable alternatives. The well-established FischerTropsch (FT) synthesis is a potential process route to produce liquid fuels and speciality chemicals and address this challenge. FT synthesis is a polymerisation reaction in which syngas, a mixture of $\mathrm{CO}$ and $\mathrm{H}_{2}$, is converted to hydrocarbon products ranging from methane to wax when low temperature conditions are used. Subsequent product upgrading steps allow high quality liquid fuels to be obtained which are clean burning. This will help to mitigate the impact of human activity on the environment.

The versatility of this process route is attributed to the ability of syngas to be generated from any carbon-containing feed such as coal, natural gas or biomass. The latter is attractive to enable a shift to a more sustainable way of living. Particularly for biomass-to-liquid plants, the high cost of syngas generation means that FT synthesis should use syngas as efficiently as possible. This requires an effective description of the $\mathrm{FT}$ reaction kinetics. This study therefore focuses on the development of a kinetic model for low temperature FT (LTFT) synthesis to improve understanding of the reaction behaviour and aid in the development of a biomass-to-liquid process route.

Although the kinetics of the $\mathrm{FT}$ reactions under the low temperature conditions of $180-260^{\circ} \mathrm{C}$ and 20-30 bar(a) have been extensively studied, the challenge to kinetic model development is the large number of possible reaction products. A common simplification is to consider the formation of the main products only, which are linear n-paraffins and 1-olefins. The polymerisation character of FT synthesis means that its product distribution could ideally be described using models based on probability theory. Deviations from probability theory distribution, however, occur especially at the conditions of LTFT synthesis. These deviations are a high methane yield, low ethene yield and the change from mainly 1-olefins at low carbon number to mainly $n$-paraffins at high carbon number. Comprehensive kinetic models in literature focus on finding a kinetic explanation for these deviations. These kinetic models, however, cannot easily be used with few being extended to include the formation of products of higher carbon number.

An aspect ignored in current kinetic model development is that FT synthesis shares many aspects of an equilibrium-controlled process. This is since $\mathrm{CO}$ hydrogenation which leads to monomer formation is the rate-determining step for the FT reactions. Consequently, the rate of chain growth is rapid in comparison. This leads to the distribution of $n$-paraffins and 1-olefins being controlled by equilibrium. By modelling $\mathrm{FT}$ synthesis as an equilibrium-controlled process, the kinetic model formulation could be simplified, consist of fewer rate expressions and contain the minimum number of model parameters without compromising on prediction quality. At the conditions of LTFT 
synthesis, both the vapour and liquid phases exist during reaction. The formation of liquid and its effect on the kinetics of FT synthesis has, however, often been neglected with most kinetic models considering the vapour phase only. This is despite of the effect that liquid formation has on product selectivity. The kinetic model developed in this thesis therefore aimed to combine the interaction between chemical equilibrium, kinetics and liquid formation and account for the formation of products of high carbon number. This will assist in providing a comprehensive description of the observed $\mathrm{FT}$ reaction behaviour in a simple and tractable manner.

A pre-requisite to kinetic model development is the creation of a physical property database for $\mathrm{n}$ paraffins and 1-olefins which is extendable to high carbon numbers. This is since only the physical properties of low carbon number n-paraffins and 1-olefins are known because they are common in most industrial processes. For chemical and phase equilibrium calculations, the critical and ideal gas properties needed to be estimated. The Constantinou Gani group contribution method, with modification to the group contributions, proved to be an effective strategy to predict the physical property data for low carbon number n-paraffins and 1-olefins. The correlations developed should therefore provide an adequate approximation of the properties of their higher carbon number relatives. To model the phase behaviour of FT synthesis, the Peng-Robinson equation of state is used. Modifications were made to the alpha function of this equation of state to ensure it remained valid when the describing the behaviour of heavy hydrocarbons.

The kinetic model development which relies on the equilibrium aspects of the reactions involved to describe the formation of $\mathrm{n}$-paraffins and 1-olefins. The reaction pathway implemented is based on the alkyl mechanism and assumes that FT synthesis can be viewed as a methylene $\left(\mathrm{CH}_{2}\right)$ polymerisation. In addition, the water gas shift (WGS) reaction is also considered. Methylene is taken as the monomer and enabled the reactions in $\mathrm{FT}$ synthesis to be represented using an equilibrium approach. Each rate expression is formulated as an equilibrium-controlled process, using species activity as the kinetic driving force. This proved to be an effective strategy to account for the observed reaction behaviour, namely a high methane yield, low ethene yield and the change from mainly 1 -olefins at low carbon number to mainly n-paraffins at higher carbon number. This approach also allowed the model to effectively capture changes in product selectivity and the product distribution as a function of process conditions ( $\mathrm{CO}$ conversion, temperature, pressure and $\mathrm{H}_{2} / \mathrm{CO}$ feed ratio). These changes could be explained by considering the equilibrium aspects of the reactions involved. The model only requires six adjustable parameters i.e. rate constants.

An important part of model development is knowing how the model rate constants determine the model output. This provides insight regarding which rate constants can be determined from the regression of data. For this purpose, a sensitivity analysis was performed on the selectivity 
to $\mathrm{C}_{1}, \mathrm{C}_{2}, \mathrm{C}_{3}, \mathrm{C}_{4}, \mathrm{C}_{5+}$ and $\mathrm{CO}_{2}$ as a function of $\mathrm{CO}$ conversion. This analysis revealed that $\mathrm{CO}$ hydrogenation is rate-determining which agrees with findings in literature. This analysis also revealed that model rate constants are cross-correlated when product selectivity as a function of $\mathrm{CO}$ conversion data is studied. This means that meaningful estimation of all rate constants using data of this form is not possible. However, it was found that when product distribution data at constant CO conversion is used instead, then meaningful estimates of the rate constants could be determined. This is if CO conversion is below 60\%. Over this CO conversion range, the product distribution is independent of the WGS reaction. The WGS rate constant should thus be approximated using data in literature. As such, five rate constants need to be determined from the regression of product distribution data.

Model validation occurred by regression of product distribution data at constant CO conversion. Emphasis was placed on the ability of the model to predict changes in the product distribution with temperature. A quantitative measure of the model fit is the precision with which the rate constants were estimated. A good fit to experimental product distributions in both fixed-bed and slurry reactors is obtained. The kinetic model has therefore been shown to be independent of reactor type. The good fit to data is quantified by the small error in the estimated rate constants, particularly for CO conversions up to approximately $30 \%$. Higher variability in the estimated rate constants was obtained for higher $\mathrm{CO}$ conversions. This emphasised the importance of estimating rate constants at conditions where the product distribution is most sensitive. The temperaturedependence of the rate constant could be described by an Arrhenius expression.

The effect of liquid formation on the kinetic behaviour of FT synthesis was modelled by assuming that the vapour and liquid phases are in equilibrium. The choice of species activity as the kinetic driving force allowed the kinetic model to be applied in both the vapour and liquid phases. Although the system was mainly in the vapour phase, liquid formation alters selectivity. Single-phase simulations are valid up to a CO conversion of $20 \%$ and predict a higher selectivity to products of carbon number in the diesel product grade $\left(C_{10}-C_{20}\right)$. Between a $C O$ conversion of $20-90 \%$, it becomes essential to account for liquid formation to ensure that the favourable selectivity to wax products $\left(\mathrm{C}_{21+}\right)$ in $\mathrm{FT}$ synthesis is adequately captured. The predictions of the single- and two-phase simulations were assessed by comparison to the wax product from LTFT reactors in Sasol processes. Both simulations were found to be useful in describing these wax product distributions.

The kinetic model developed in this thesis therefore effectively describes the behaviour of FT synthesis. The ability of the model to predict changes in product selectivity and the product distribution as a function of process conditions will make it a powerful tool involved in the design of FT processes. It is recommended that the approach taken to develop the model be used to study the kinetics of other gas-to-liquid processes, for reactor design and flowsheet development. 


\section{Declaration}

I know the meaning of plagiarism and declare that all the work in this thesis, save for that which is properly acknowledged, is my own. This thesis/dissertation has been submitted to the Turnitin module (or equivalent similarity and originality checking software) and I confirm that my supervisor has seen my report and any concerns revealed by such have been resolved with my supervisor.

I hereby grant the University of Cape Town free license to reproduce, for the purpose of research, either the whole or any portion of the contents in any manner whatsoever of the thesis.

I declare that the work presented in this thesis is my own unaided work, both in conception and execution and apart from the guidance of my supervisor, I have received no assistance. Neither the substance or any part of this thesis has been submitted in the past for a degree at the University of Cape Town or any other university.

I am now presenting this thesis for examination for the degree of Master of Science in Chemical Engineering.

\section{Signed by candidate}

Muhammad Imaad Davies 


\section{Acknowledgements}

In the name of Allah, Most Gracious, Most Merciful.

"And those who strive In Our (Cause), - We will Certainly guide them to our Paths:

For verily Allah is with those who do right." (The Holy Qur'an, Surah 29:69)

All praise is due to Allah for providing me with the opportunity to study further and gain knowledge as an act of worship. This thesis has shown that this will not always be easy but that going through difficulty is an important part of becoming a better Muslim. Knowledge is one of the paths open to the man to attain closeness to Allah. It has helped to strengthen my Imaan (faith) and tawakkul (trust in God). I am grateful for this thesis being a means to continue in the Way of God (sirāt-ul-Mustaqeem).

To my supervisor, Prof. Klaus Möller, who initiated the project and then allowed me to develop my own ideas and approach to the problem. Your guidance was an essential ingredient in enabling this project to come together. I thank you for all the opportunities you have given me since the final year of my undergraduate studies in 2018. It has been a pleasure to have been taught by and learn from you.

To Chelsea Tucker, who taught me so much about Fischer-Tropsch synthesis, assisted me in any way and provided the inspiration and enthusiasm to develop the model in this thesis, all while she was finalising her own Ph.D thesis. I hope this work may be of use to you in your future process development. To Dave Wright, thank you for being my mentor and for all your guidance.

Thank you to my colleagues in the loft of the chemical engineering building for being comic relief, entertainment and support, especially when we were meant to be working but were hardly working. To Bongani and my fellow process modellers, Evren and Vidushi, I am grateful for all your support, encouragement and positivity. To my friends and family, I thank you for your interest, enthusiasm and belief. I am especially grateful to my siblings for their unique way to make life interesting.

To my parents, Salama and Rafiq, you have given me every opportunity in life, seen me through every setback and provided me with the foundation to pursue anything. Without you both it would not have been possible to make this thesis a success, especially during the last few months. I am grateful and pray that Allah blesses you both abundantly. To my partner in crime, Farhana, thank you for living this thesis with me. Your support, encouragement and love made working on this thesis each day worth it. I am blessed to have found someone who makes everything in life an adventure.

Financial assistance from the National Research Foundation (NRF) is appreciated. 


\section{Contents}

1 Introduction 1

1.1 Background . . . . . . . . . . . . . . . . . . . . . . . . . . . . . . . 1

1.2 Problem Solving Methodology $\ldots \ldots \ldots \ldots$

$\begin{array}{lll}2 & \text { Literature Review } & \mathbf{7}\end{array}$

2.1 Fischer-Tropsch Chemistry and Catalysis . . . . . . . . . . . . . . . . . . 7

2.1 .1 Fischer-Tropsch Reactions . . . . . . . . . . . . . . . . . 7

2.1 .2 Fischer-Tropsch Catalysts . . . . . . . . . . . . . . . . . . . . . 8

2.1 .3 Fischer-Tropsch Reaction Mechanism . . . . . . . . . . . . . . . . . 10

2.1 .4 Product Selectivity . . . . . . . . . . . . . . . . . . . . . . . . . . 12

2.1 .5 Possible Causes for Deviations from the ASF Distribution . . . . . . . . . 15

$2.1 .5 .1 \quad$ High Methane Yield . . . . . . . . . . . . . . . . . . . . . . . 15

2.1 .5 .2 Low Ethene Yield and Change in $\alpha$. . . . . . . . . . . . . 15

2.2 Kinetic Modelling . . . . . . . . . . . . . . . . . . . . . . . . . . . . . . . . . . . . . 17

2.2 .1 Overall Fischer-Tropsch Reaction Rate and Selectivity Models . . . . . . . 17

2.2 .2 Fischer-Tropsch Mechanistic Kinetic Models . . . . . . . . . . . . . . 18

2.2.3 Modelling Carbon Number Dependent Processes affecting Secondary Reactions . . . . . . . . . . . . . . . . . . . . . . 20

2.2.3.1 1 1-Olefin Carbon Number Dependent Diffusion . . . . . . . . . . 21

2.2 .3 .2 1-Olefin Carbon Number Dependent Physisorption . . . . . . . . 22

2.2.3.3 1-Olefin Carbon Number Dependent Solubility/Vapour-Liquid Equilibrium . . . . . . . . . . . . . . . . . . . 23

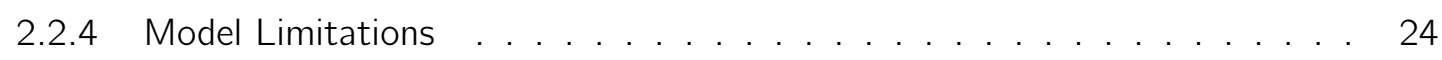

2.2 .5 Modelling Vapour-Liquid Equilibrium (VLE) . . . . . . . . . . . . . . 26

2.2 .6 Chemical Equilibrium Considerations . . . . . . . . . . . . . . . . . . . 28

2.2 .7 Influence of Process Conditions on Selectivity . . . . . . . . . . . . 30

2.3 Summary and Insights Gained $\ldots \ldots \ldots \ldots$ 
\begin{tabular}{lll}
\hline & Thermo-physical Considerations & 35
\end{tabular}

3.1 Physical Property Estimation . . . . . . . . . . . . . . . . . . . . . . . . . . . . 35

3.1 .1 Theory . . . . . . . . . . . . . . . . . . . . . . . . . . . . 35

3.1 .2 Methodology and Assumptions . . . . . . . . . . . . . . . . 36

3.1 .3 Results and Discussion . . . . . . . . . . . . . . . . . . . . . . 38

3.1.3.1 Critical Pressure Estimation . . . . . . . . . . . . . . . . 38

3.1 .3 .2 Critical Temperature Estimation . . . . . . . . . . . . . . . . 39

3.1.3.3 Normal Boiling Point Estimation . . . . . . . . . . . . . . . . 40

3.1.3.4 Critical Volume Estimation . . . . . . . . . . . . . . . . . . . . 41

3.1.3.5 Acentric Factor Estimation . . . . . . . . . . . . . . . . . . . . 42

3.1.3.6 Heat and Gibbs Free Energy of Formation Estimation . . . . . . 44

3.1.3.7 Ideal Gas Heat Capacity Estimation . . . . . . . . . . . . . . . . 45

3.2 Thermodynamic Modelling . . . . . . . . . . . . . . . . . . . . . . . . . . . . . . . . . 48

3.2 .1 Choice of Mixing Rule . . . . . . . . . . . . . . . . . . . . . . . . . . . . 49

3.2 .2 Choice of Alpha Function . . . . . . . . . . . . . . . . . . 50

4 Model Development 53

4.1 Partial Equilibrium Approach to Kinetic Modelling . . . . . . . . . . . . . . . . . 54

4.2 Model Formulation . . . . . . . . . . . . . . . . . . . . . . . . . . . 55

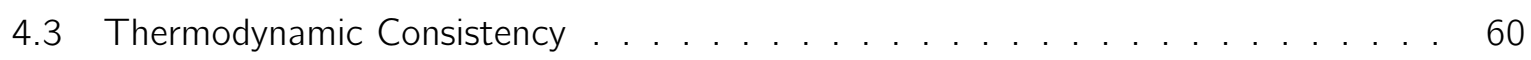

4.4 Model Demonstration . . . . . . . . . . . . . . . . . . . . . . . . . . . . . . . . . . . . . . . . 62

4.5 Effect of Operating Conditions . . . . . . . . . . . . . . . . . . . 66

4.5 .1 Effect of CO Conversion . . . . . . . . . . . . . . . . 67

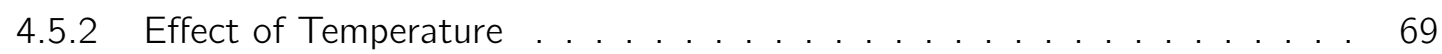

4.5 .3 Effect of Pressure . . . . . . . . . . . . . . . . . . . . . . . . . . . . . . . . . 71

4.5 .4 Effect of $\mathrm{H}_{2} / \mathrm{CO}$ Feed Ratio . . . . . . . . . . . . . . . . . 72

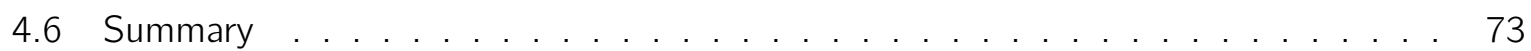

$\begin{array}{lll}5 & \text { Sensitivity analysis } & \mathbf{7 5}\end{array}$

5.1 Sensitivity Analysis Approaches . . . . . . . . . . . . . . . . . . . . . . . . 76

5.1 .1 One-at-a-time Approach . . . . . . . . . . . . . . . . . . 76

5.1 .2 Derivative-Based Local Approach . . . . . . . . . . . . . . . . 77

5.2 One-at-a-time Analysis $\ldots \ldots \ldots \ldots$. . . . . . . . . . . . . . . . . 78

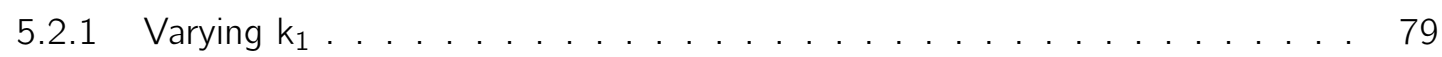

5.2 .2 Varying $k_{2} \ldots \ldots \ldots \ldots \ldots$

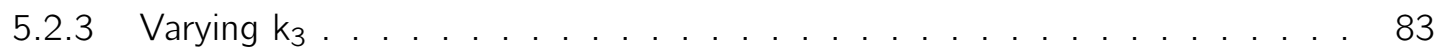

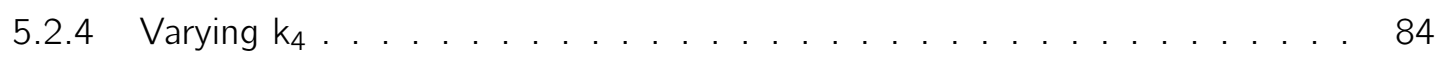


5.2 .5 Varying $k_{5} \ldots \ldots \ldots \ldots \ldots \ldots \ldots$

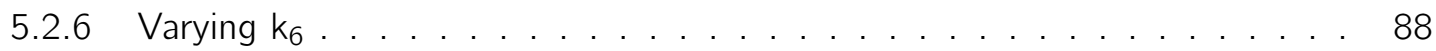

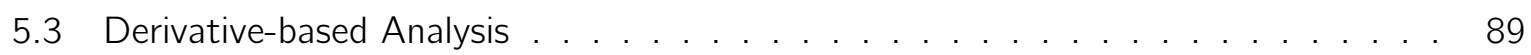

5.3 .1 Sensitivity function of $C_{1}$ with respect to $k_{j} \ldots \ldots . \ldots 90$

5.3 .2 Sensitivity function of $C_{2}$ with respect to $k_{j} \ldots \ldots . \ldots . \ldots 92$

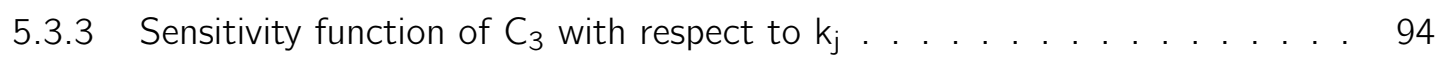

5.3 .4 Sensitivity function of $C_{4}$ with respect to $k_{j} \ldots \ldots \ldots . \ldots . \ldots 95$

5.3 .5 Sensitivity function of $C_{5+}$ with respect to $k_{j} \ldots \ldots . \ldots 97$

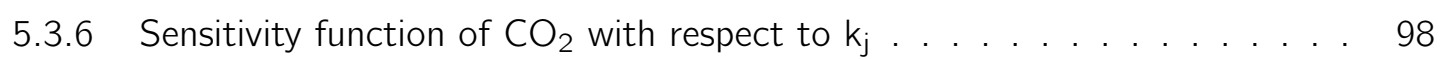

5.3 .7 Key Features . . . . . . . . . . . . . . . . . . . . . . . . . . . . . . . . . . . . . . 99

5.3.8 Product Distribution Sensitivity with respect to each $\mathrm{k}_{\mathrm{j}}$. . . . . . . . . . 102

5.4 Summary $\ldots \ldots \ldots \ldots \ldots \ldots$

$\begin{array}{lll}6 & \text { Model Validation } & 107\end{array}$

6.1 Introduction . . . . . . . . . . . . . . . . . . . . . . 107

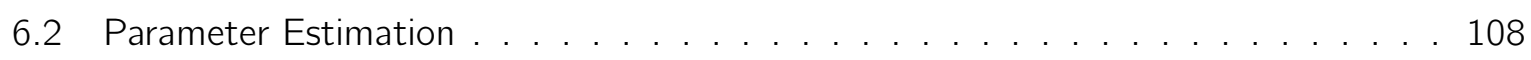

6.3 Solving Procedure $\ldots \ldots \ldots \ldots$. . . . . . . . . . . . . . . . . . . . . . . . . . . . . . . 110

6.3 .1 Method of Solution . . . . . . . . . . . . . . . . . . . . 111

6.3 .2 Model Assumptions . . . . . . . . . . . . . . . . . . . . . . . . . 114

6.4 Results and Discussion: Fixed-Bed Reactor Data . . . . . . . . . . . . . . . . . 115

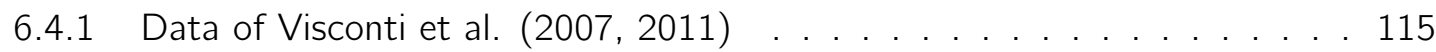

6.4 .2 Data of Visconti et al. (2009) . . . . . . . . . . . . . . . . . . . 118

6.5 Results and Discussion: Slurry Reactor Data . . . . . . . . . . . . . . . 120

6.5 .1 Data of Visconti et al. (2016) . . . . . . . . . . . . . . . . 120

6.5 .2 Data of Tucker \& van Steen (2019) . . . . . . . . . . . . . . 122

6.6 Rate Constant Temperature Dependence . . . . . . . . . . . . . . . . . . . 123

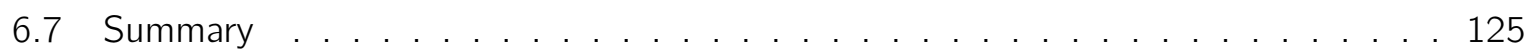

$\begin{array}{lll}7 & \text { Two-Phase Considerations } & 127\end{array}$

7.1 Liquid Phase Formation in Fischer-Tropsch Synthesis . . . . . . . . . . . . . . 127

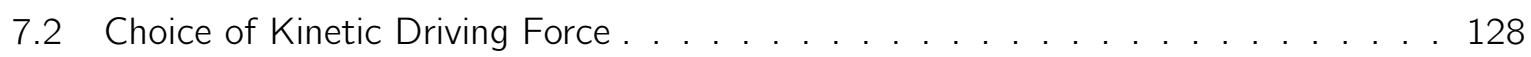

7.3 Methodology . . . . . . . . . . . . . . . . . . . . . . . . . . . . . . . . . . . . . . . . . . 129

7.4 Results and Discussion $\ldots \ldots \ldots \ldots \ldots$

7.4 .1 Influence of Vapour-Liquid Equilibrium . . . . . . . . . . . . . . 133

7.4.2 Effect of CO Conversion on the Single- and Two-phase Simulations . . . . 136

7.5 Comparison to Experimental Data . . . . . . . . . . . . . . . . . . . . 142

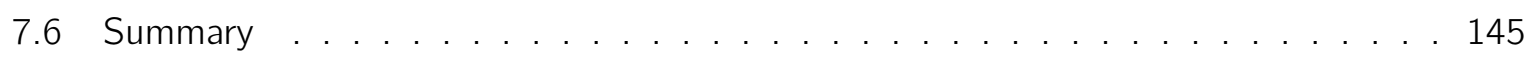


8 Conclusions and Recommendations 147

8.1 Conclusions . . . . . . . . . . . . . . . . . . . . . . . . . . . . . . . . 147

8.2 Recommendations. . . . . . . . . . . . . . . . . . . . . . 150

8.2 .1 Reaction Kinetics . . . . . . . . . . . . . . . . . . . . . 150

8.2 .2 Reactor Design . . . . . . . . . . . . . . . . . . . . . . 151

8.2 .3 Process Flowsheet Development . . . . . . . . . . . . . . . . . . . 151

\begin{tabular}{ll}
\hline Bibliography & 153
\end{tabular}

\begin{tabular}{|ll}
\hline A Validation of Model Development Assumptions & 163
\end{tabular}

A.1 Validation of the Pseudo-Steady State Assumption . . . . . . . . . . 163

A.2 Validation of the Choice of Maximum Carbon Number . . . . . . . . . . . . 165

A.3 Equilibrium Constants as a Function of Temperature . . . . . . . . . . . . . 166

\begin{tabular}{ll}
\hline B Sensitivity Calculations & 169
\end{tabular}

\begin{tabular}{ll}
\hline$C$ The Need for an Equation of State & 171
\end{tabular} 


\section{List of Figures}

1.1 Configuration of the fixed-bed reactor $(A)$ and slurry reactor $(B)$ used for LTFT synthesis adapted from Espinoza et al. (1999)]. . . . . . . . . . . . . 2

2.1 Productivity comparison between a commercial Sasol precipitated Fe catalyst $\left(240^{\circ} \mathrm{C}\right)$ and a commercial Sasol supported Co catalyst $\left(220^{\circ} \mathrm{C}\right)$ adapted from Espinoza

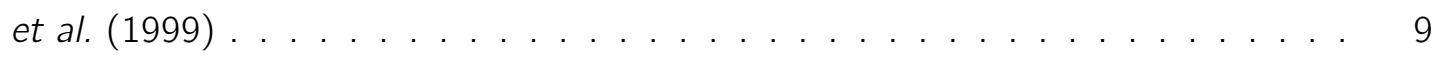

2.2 Initiation, chain growth and termination steps in the alkyl mechanism adapted from Claeys \& van Steen[(2004)] . . . . . . . . . . . . . . . . . . . . . . 11

2.3 Weight fraction of the total hydrocarbon distribution as a function of $\alpha$ using

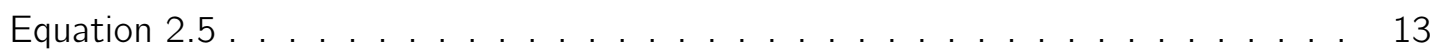

2.4 Molar product distribution and olefin-to-paraffin ratio as a function of carbon number in LTFT synthesis. Data from Iglesia et al. (1993) at $203^{\circ} \mathrm{C}, 6$ bar(a) and $\mathrm{H}_{2} / \mathrm{CO}$ feed ratio of 2.1 for a $\mathrm{Ru} / \mathrm{TiO}_{2}$ catalyst. . . . . . . . . . . . . . . . 14

2.5 Chain growth probability as a function of carbon number for different catalysts

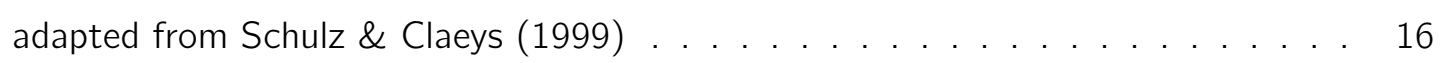

2.6 Physisorption of the n-paraffins as a function of carbon number at $233^{\circ} \mathrm{C}$ and 80 bar(a), using n-heptane as a reference. Data from Laxmi Narasimhan et al.[(2006). 23

\begin{tabular}{|ll}
\hline 2.7 Sum of the product weight fractions as a function of $\alpha$ predicted by Equation 2.5 & \\
\hline
\end{tabular} adapted from Khazali (2018) . . . . . . . . . . . . . . . . . . 25

2.8 Molar ASF distribution derived from thermodynamics at $327^{\circ} \mathrm{C}$ and 10 bar(a) adapted from Norval \& Phillips[(1990)] . . . . . . . . . . . . . . . . . . 29

3.1 Critical pressure prediction by the CG method . . . . . . . . . . . . . . . . . . 39

3.2 Critical temperature prediction by the CG method . . . . . . . . . . . . . . . 40

3.3 Normal boiling point prediction by the CG method . . . . . . . . . . . . . . . 41

3.4 Critical volume prediction by the CG method . . . . . . . . . . . . . . . . . 42

3.5 Acentric factor prediction by the CG method . . . . . . . . . . . . . . . . 43

3.6 Heat of formation at $298.15 \mathrm{~K}$ prediction by the CG method . . . . . . . . . . . 44 
3.7 Gibbs free energy of formation at $298.15 \mathrm{~K}$ prediction by the CG method . . . . . 45

3.8 Ideal gas heat capacity prediction by the CG method. The black arrow indicates the direction of increasing temperature. . . . . . . . . . . . . . . . . . . 46

3.9 Syngas components, methane and ethene ideal gas heat capacity prediction. . . . 47

3.10 Comparison of the different alpha functions used in the PR-EOS applied to the

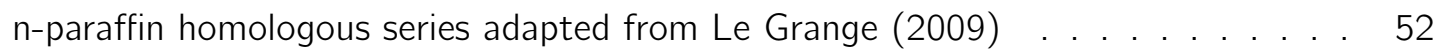

4.1 Proposed FT reaction pathway adapted from Fernandes $[(2005)$ and Schulz et al. $\square$ (1994) . . . . . . . . . . . . . . . . . . . . . . 56

$4.2 \quad \mathrm{~N}$-paraffin and 1-olefin product distribution and olefin-to-paraffin ratio as a function of carbon number at a CO conversion of $10 \%, 220^{\circ} \mathrm{C}, 20$ bar(a) and $\mathrm{H}_{2} / \mathrm{CO}$ feed

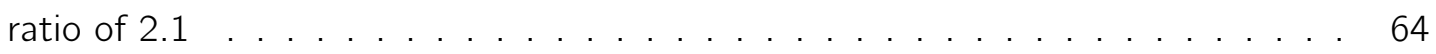

4.3 Semi-logarithmic plot of the hydrocarbon distribution and chain growth probability, both as functions of carbon number at a CO conversion of $10 \%, 220^{\circ} \mathrm{C}, 20$ bar(a) and $\mathrm{H}_{2} / \mathrm{CO}$ feed ratio of $2.1 \ldots \ldots \ldots \ldots . \ldots . \ldots . \ldots 65$

4.4 The effect of CO conversion on the total hydrocarbon, $\mathrm{n}$-paraffin and 1-olefin product distributions and carbon-based selectivity at $220^{\circ} \mathrm{C}, 20$ bar(a) and an $\mathrm{H}_{2} / \mathrm{CO}$

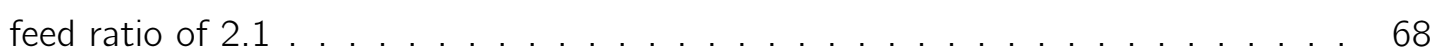

4.5 The effect of temperature on the total hydrocarbon, $n$-paraffin and 1-olefin product distributions and carbon-based selectivity at a CO conversion of 40\%, 20 bar(a) and an $\mathrm{H}_{2} / \mathrm{CO}$ feed ratio of $2.1 \ldots \ldots \ldots 70 \ldots$

4.6 The effect of pressure on the total hydrocarbon, n-paraffin and 1-olefin product distributions and carbon-based selectivity at a CO conversion of $40 \%, 220^{\circ} \mathrm{C}$ and an $\mathrm{H}_{2} / \mathrm{CO}$ feed ratio of $2.1 \ldots \ldots \ldots 71$

4.7 The effect of feed ratio on the total hydrocarbon, n-paraffin and 1-olefin product distributions and carbon-based selectivity at a CO conversion of $40 \%, 220^{\circ} \mathrm{C}$ and

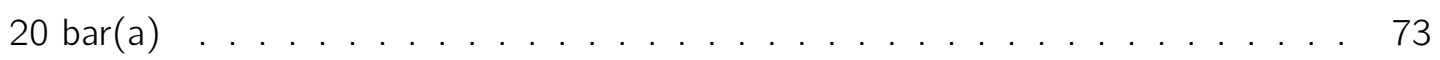

5.1 The effect of variations of $k_{1}$ on the carbon-based selectivity at $220^{\circ} \mathrm{C}, 20$ bar(a) and $\mathrm{H}_{2} / \mathrm{CO}$ feed ratio of $2.1 \ldots \ldots \ldots \ldots$. . . . . . . . . . . . . . . . . . . .

5.2 The effect of variations of $k_{1}$ on the carbon-based selectivity at $220^{\circ} \mathrm{C}, 20$ bar(a) and $\mathrm{H}_{2} / \mathrm{CO}$ feed ratio of 2.1 continued $\ldots \ldots . \ldots . \ldots . . \ldots 8$

5.3 The effect of variations of $k_{2}$ on the carbon-based selectivity at $220^{\circ} \mathrm{C}, 20 \mathrm{bar}(\mathrm{a})$

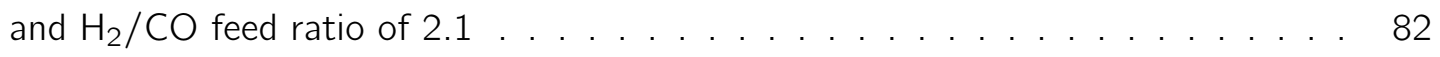

5.4 The effect of variations of $k_{3}$ on the carbon-based selectivity at $220^{\circ} \mathrm{C}, 20 \mathrm{bar}(\mathrm{a})$

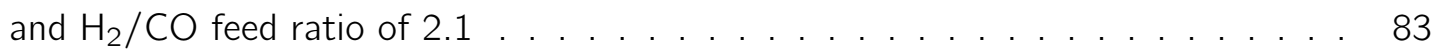


5.5 The effect of variations of $k_{3}$ on the carbon-based selectivity at $220^{\circ} \mathrm{C}, 20 \mathrm{bar}(\mathrm{a})$

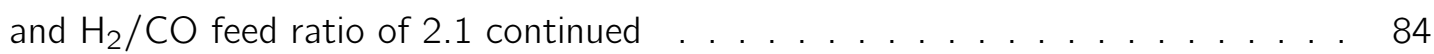

5.6 The effect of variations of $k_{4}$ on the carbon-based selectivity at $220^{\circ} \mathrm{C}, 20$ bar(a) and $\mathrm{H}_{2} / \mathrm{CO}$ feed ratio of $2.1 \ldots \ldots \ldots \ldots \ldots$

5.7 The effect of variations of $k_{4}$ on the carbon-based selectivity at $220^{\circ} \mathrm{C}, 20$ bar(a) and $\mathrm{H}_{2} / \mathrm{CO}$ feed ratio of 2.1 continued . . . . . . . . . . . . . 86

5.8 The effect of variations of $k_{5}$ on the carbon-based selectivity at $220^{\circ} \mathrm{C}, 20$ bar(a)

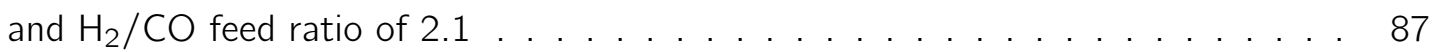

5.9 The effect of variations of $k_{6}$ on the carbon-based selectivity at $220^{\circ} \mathrm{C}, 20$ bar(a)

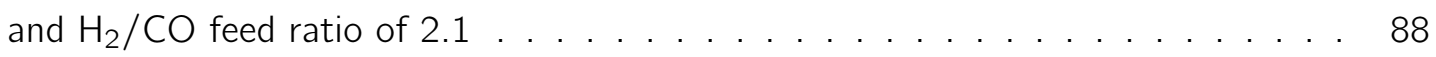

5.10 The effect of variations of $k_{6}$ on the carbon-based selectivity at $220^{\circ} \mathrm{C}, 20 \mathrm{bar}(\mathrm{a})$ and $\mathrm{H}_{2} / \mathrm{CO}$ feed ratio of 2.1 continued . . . . . . . . . . . . . . . . . . 89

5.11 Sensitivity of $C_{1}$ with respect to each $\mathrm{k}_{\mathrm{j}} \ldots \ldots \ldots \ldots$

5.12 Sensitivity of $C_{2}$ with respect to each $k_{j} \ldots \ldots \ldots . \ldots . \ldots . \ldots 9$

5.13 Sensitivity of $C_{3}$ with respect to each $\mathrm{k}_{\mathrm{j}} \ldots \ldots \ldots \ldots \ldots$

5.14 Sensitivity of $\mathrm{C}_{4}$ with respect to each $\mathrm{k}_{\mathrm{j}} \ldots \ldots \ldots \ldots$. . . . . . . . 96

5.15 Sensitivity of $C_{5+}$ with respect to each $\mathrm{k}_{\mathrm{j}} \ldots \ldots \ldots \ldots$

5.16 Sensitivity of $\mathrm{CO}_{2}$ with respect to each $\mathrm{k}_{\mathrm{j}} \ldots \ldots \ldots \ldots$. . . . . . . . . 99

5.17 Linear dependence between the ratios of the sensitivity functions with respect to the rate constants . . . . . . . . . . . . . . . . . . . . . . . . . . 101

5.18 Sensitivity of the product distribution with respect to $\mathrm{k}_{\mathrm{j}}$ as a function of $\mathrm{CO}$ conversion at $220^{\circ} \mathrm{C}, 20 \mathrm{bar}(\mathrm{a})$ and $\mathrm{H}_{2} / \mathrm{CO}$ feed ratio of 2.1. . . . . . . . . . . . . 104

6.1 Solution process followed to regress the experimental data and determine values of

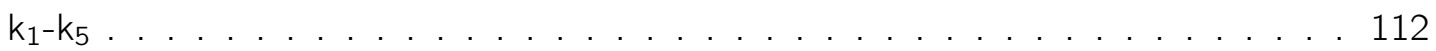

6.2 Model fit to the data of Visconti et al. [2007, 2011) at a GHSV of $5000 \mathrm{Nml} / \mathrm{g}_{\text {cat }} / \mathrm{h} 116$

6.3 Model fit to the data of Visconti et al. [2009) at a GHSV of $4800 \mathrm{Nml} / \mathrm{g}_{\text {cat }} / \mathrm{h}$. . 119

6.4 Model fit to the data of Visconti et al. [2016) at a GHSV of $2000 \mathrm{Nml} / \mathrm{g}_{\text {cat }} / \mathrm{h}$. . 120

6.5 Model fit to the data of Tucker \& van Steen[(2019) . . . . . . . . . . . . . . 122

6.6 Fit of the rate constants resulting from the data of Visconti et al.](2007,,2011) to the Arrhenius expression. Dashed lines represent the model prediction . . . . . . . 124

7.1 Outline of the solution process used in the two-phase FBR model . . . . . . . . 131

7.2 Comparison of the vapour phase and vapour-liquid product distributions at a $\mathrm{CO}$ conversion of $60 \%, 220^{\circ} \mathrm{C}, 20 \mathrm{bar}(\mathrm{a})$ and $\mathrm{H}_{2} / \mathrm{CO}$ feed ratio of $2.1 \ldots . . . .133$

7.3 Influence of VLE on the FT product distribution at a CO conversion of $60 \%, 220^{\circ} \mathrm{C}$,

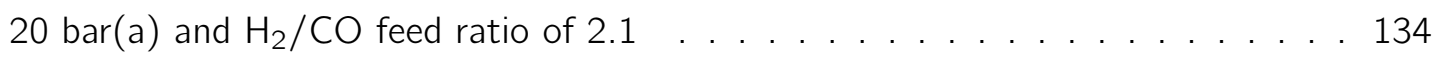


7.4 Influence of VLE on the product selectivity as a function of CO conversion at $220{ }^{\circ} \mathrm{C}, 20 \operatorname{bar}(\mathrm{a})$ and $\mathrm{H}_{2} / \mathrm{CO}$ feed ratio of $2.1 \ldots \ldots . . \ldots 137$

7.5 Vapour fraction as a function of $\mathrm{CO}$ conversion at $220^{\circ} \mathrm{C}, 20 \mathrm{bar}(\mathrm{a})$ and $\mathrm{H}_{2} / \mathrm{CO}$

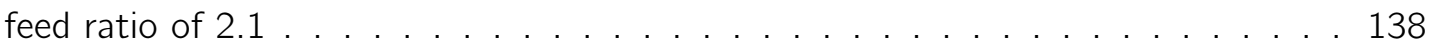

7.6 Comparison of the total hydrocarbon product distributions as a function of $\mathrm{CO}$ conversion at $220^{\circ} \mathrm{C}, 20 \mathrm{bar}(\mathrm{a})$ and $\mathrm{H}_{2} / \mathrm{CO}$ feed ratio of 2.1. . . . . . . . . . . 140

7.7 Comparison of the n-paraffin and 1-olefin product distributions as a function of $\mathrm{CO}$ conversion at $220^{\circ} \mathrm{C}, 20 \mathrm{bar}(\mathrm{a})$ and $\mathrm{H}_{2} / \mathrm{CO}$ feed ratio of $2.1 . \ldots . . . . . .141$

7.8 Model comparison to the wax product from Sasol LTFT reactors (Leckel, 2005)] . 142

7.9 Model comparison to Sasol LTFT M5 wax (Leckel \& Liwanga-Ehumbu, 2006)] . . 143

A.1 Validation of the pseudo-steady state approximation applied to $\mathrm{CH}_{2} \ldots \ldots$. . . 164

A.2 Simulation of the FT polymerisation behaviour using $N=81$ and $N=121 \ldots 165$

A.3 Equilibrium constants as function of temperature . . . . . . . . . . . . . 166

B.1 Sensitivity functions for the $\mathrm{C}_{1}, \mathrm{C}_{2}, \mathrm{C}_{3}, \mathrm{C}_{4}, \mathrm{C}_{5+}$ and $\mathrm{CO}_{2}$ calculated using the second order finite difference approximation. . . . . . . . . . . . . . . . 170

C.1 Selectivity comparison assuming ideal phases and using the PR-EOS at $220^{\circ} \mathrm{C}, 20$

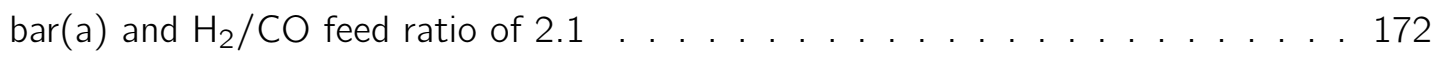

C.2 Vapour fraction comparison assuming ideal phases and using the PR-EOS at $220^{\circ} \mathrm{C}$, $20 \mathrm{bar}(\mathrm{a})$ and $\mathrm{H}_{2} / \mathrm{CO}$ feed ratio of $2.1 \ldots \ldots \ldots$ 


\section{List of Tables}

2.1 Proposed reactions in the alkyl mechanism adapted from van der Laan (1999), Visconti et al. $[(2007)$ and Visconti et al.[(2011) . . . . . . . . . . . . . . . . . . 19

2.2 Strategies used by literature kinetic models to incorporate carbon number (CN) dependent processes . . . . . . . . . . . . . . . . . . . . . . . . . 21

3.1 Critical pressure parameters in Equation $3.5 \ldots \ldots \ldots \ldots$

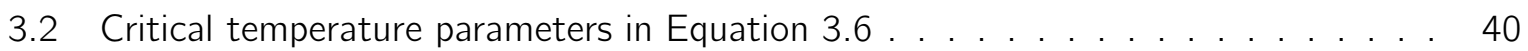

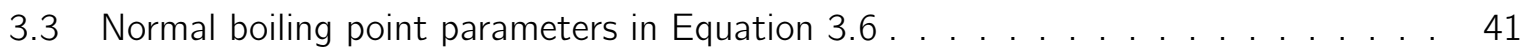

3.4 Critical volume parameters in Equation $3.7 \ldots \ldots \ldots$

3.5 Acentric factor parameters in Equation $3.10 \ldots \ldots$. . . . . . . . . . . 44

3.6 Ideal gas formation property parameters in Equation 3.11 and/3.12 $\ldots \ldots \ldots$

3.7 Ideal gas heat capacity parameters in Equation $3.13 \ldots \ldots \ldots$

\begin{tabular}{|lll|}
\hline 3.8 & Syngas components, methane and ethene heat capacity parameters in Equation 3.14 & 47
\end{tabular}

4.1 Chemical reactions in the proposed reaction pathway . . . . . . . . . . . . . 56

4.2 Rate expressions for the reaction steps in the proposed reaction pathway . . . . . 57

4.3 Net reactions at complete chemical equilibrium . . . . . . . . . . . . . . . . 62

4.4 Numerical values of the rate constants in reaction step 1-6 . . . . . . . . . . . 63

4.5 Element balance closure from model demonstration . . . . . . . . . . . . . . . . 66

5.1 Numerical values of the base case rate constants in reaction step 1-6 . . . . . . . 78

5.2 Regression constants in Equation 5.5 from the linear dependence of the ratio of sensitivity functions . . . . . . . . . . . . . . . . . . . . 100

6.1 Summary of experimental data used to estimate the model rate constants . . . . . 108

6.2 Pre-exponential factor and activation energy used to approximate $k_{6}$ (Rafiq et al.,

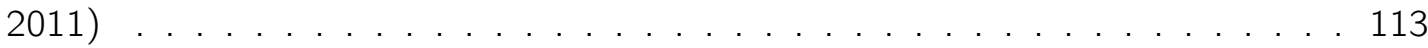


6.3 Comparison between the experimental and model predicted $\mathrm{CO}$ conversion and estimated vapour fraction for the data of Visconti et al.[(2007, 2011)] . . . . . . . 117

6.4 Summary of the estimated rate constants resulting from the data of (Visconti et al. 2007, 2011) . . . . . . . . . . . . . . . . . . . . . . . . . . . 118

6.5 Comparison between the experimental and model predicted CO conversion and estimated vapour fraction for the data of Visconti et al.[(2009)] . . . . . . . . . . . 119

6.6 Summary of the estimated rate constants resulting from the data of (Visconti et al.

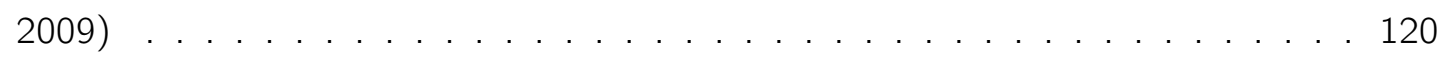

6.7 Comparison between the experimental and model predicted $\mathrm{CO}$ conversion and estimated vapour fraction for the data of Visconti et al.[(2016)] . . . . . . . . . . . 121

6.8 Summary of the estimated rate constants resulting from the data of Visconti et al. (2016) . . . . . . . . . . . . . . . . . . . . . . . . . 121

6.9 Summary of the rate constants used to estimate the selectivity profiles of Tucker

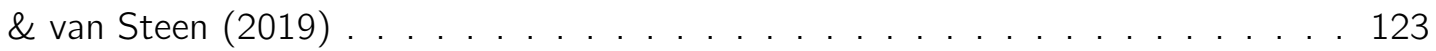

6.10 Estimated pre-exponential factors and activation energies for $k_{1}-k_{5} \ldots . . . . .125$

7.1 Rate constants for reaction step 1-6 in the single- and two-phase models . . . . 130

7.2 Definition of the different product grades obtained from crude oil refinery processes 136

7.3 Model rate constants when compared to the wax product from Sasol processes . . 144 


\section{Nomenclature}

\begin{tabular}{|c|c|}
\hline$n$ & Number of carbon atoms \\
\hline$m$ & $\begin{array}{l}\text { Number of hydrogen atoms in hydrocarbon product/number of } \\
\text { model parameters }\end{array}$ \\
\hline$\alpha$ & $\begin{array}{l}\text { Chain growth probability/function representing pure component } \\
\text { vapour pressure }\end{array}$ \\
\hline$w_{n}$ & Weight fraction of product with $n$ carbon atoms \\
\hline$y_{n}$ & Mole fraction of product with $n$ carbon atoms \\
\hline$S$ & Active site on the catalyst surface \\
\hline$E_{\text {desorption }}$ & Activation energy for 1-olefin desorption \\
\hline$k_{\text {desorption }}$ & Rate constant for 1-olefin desorption \\
\hline$x_{O_{n}}$ & Mole fraction of 1-olefins in the liquid phase \\
\hline$P_{O_{n}}$ & Partial pressure of 1-olefins in the vapour phase \\
\hline$\beta$ & Arbitrary constant \\
\hline$\gamma$ & Arbitrary constant \\
\hline$n_{\text {species }}$ & Number of species in the reaction mixture \\
\hline$K_{i}$ & Separation factor \\
\hline$z_{i}$ & Overall mole fraction of species $i$ \\
\hline$\psi$ & Total molar vapour fraction \\
\hline$a_{i}$ & Activity of species $i$ /arbitrary constant \\
\hline$f_{i} / f_{i}^{\circ}$ & Fugacity of species $i$, reference state fugacity of species $i$ \\
\hline$T$ & Temperature \\
\hline$P$ & Pressure \\
\hline$\lambda_{i}$ & Lagrange multiplier for element $i$ \\
\hline$n_{\text {groups }}$ & Number of UNIFAC groups present in a molecule \\
\hline$F_{i j}$ & Contribution of the group of order $i$ for property $j$ \\
\hline$W$ & Second order group weighting/Catalyst weight \\
\hline$n_{\text {data }}$ & Number of data points \\
\hline$Y_{\text {exp,i }}$ & Experimental data $i$ \\
\hline$Y_{\text {calc }, i}$ & Calculated value $i$ \\
\hline$P_{c}$ & Critical pressure \\
\hline$T_{c}$ & Critical temperature \\
\hline$T_{b}$ & Normal boiling point \\
\hline
\end{tabular}


$V_{c} \quad$ Critical volume

$\left[\mathrm{m}^{3} / \mathrm{kmol}\right]$

$\omega \quad$ Acentric factor

$P_{i}^{\text {sat }} \quad$ Saturated vapour pressure

$[\operatorname{bar}(a)]$

V Molar volume

VSTP Molar volume at standard temperature $(273.15 \mathrm{~K})$ and pressure (1 $\left[\mathrm{m}^{3} / \mathrm{mol}\right]$ $\operatorname{bar}(\mathrm{a}))$

$k_{i j} \quad$ Binary interaction parameter

$K_{a, n} \quad$ Activity-based equilibrium constant for reaction $n$

$\Delta G_{r \times n}^{\circ} \quad$ Ideal gas Gibbs free energy of reaction at 1 bar(a) and temperature $T$

$r_{i} \quad$ Rate of reaction for species $i /$ residual

$k_{j} \quad$ Rate constant for reaction step $j$

Wotal Total catalyst weight

$N \quad$ Highest carbon number present in the simulation

$[\mathrm{mol} / \mathrm{kg} / \mathrm{s}] /[-]$ $[\mathrm{mol} / \mathrm{kg} / \mathrm{s}]$

$F_{i} \quad$ Flow rate of species $i$

$F_{i, 0} \quad$ Feed flow rate of species $i$

$X_{C O} \quad$ CO conversion $[\mathrm{mol} / \mathrm{s}]$

$S_{i} \quad$ Carbon-based selectivity

$f \quad$ (Objective) function

$\Delta k_{j} \quad$ Incremental change in rate constant

$s_{i j} \quad$ Relative sensitivity of the selectivity $i$ with respect to rate constant j

$b_{i} \quad$ Arbitrary constant

$\hat{\sigma}^{2} \quad$ Residual variance

cov Covariance matrix

J Jacobian (matrix of first derivatives

se $\left(\hat{k}_{j}\right) \quad$ Standard error in rate constant estimate

$\epsilon_{\text {mach }}$ Machine precision

$A_{j} \quad$ Pre-exponential factor

$[\mathrm{mol} / \mathrm{kg} / \mathrm{s}]$

$E_{a, j} \quad$ Activation energy for reaction step $j$

$[\mathrm{kJ} / \mathrm{mol}]$

GHSV Gas hourly space velocity 


\section{Chapter 1}

\section{Introduction}

\subsection{Background}

Globally, there is a need to replace our dependence on fossil fuels as the main source of energy. This will aid in reducing the impact of human activity on the environment, address issues of energy supply and security and facilitate a shift towards a more sustainable way of living. To achieve these goals, it is necessary to replace fossil fuels with renewable and environmentally friendly alternatives. This has led to an increased interest in the well-established Fischer-Tropsch synthesis.

Fischer-Tropsch (FT) synthesis is a gas-to-liquid process route for the conversion of syngas, a mixture of $\mathrm{CO}$ and $\mathrm{H}_{2}$, to liquid fuels and speciality chemicals. It is a versatile technology because syngas can be produced from any carbon-containing feed such as coal, natural gas or biomass (Lozano-Blanco et al., 2006; van Steen et al., 2018; Hensen et al., 2020). FT synthesis is a polymerisation reaction which involves the hydrogenation of $\mathrm{CO}$ to produce hydrocarbon products ranging from methane to wax, in the presence of a metal catalyst. The reactions of $F T$ synthesis can be concisely represented by Reaction 1.1, where $n$ and $m$ are the average number of carbon and hydrogen atoms in the hydrocarbon product (van der Laan, 1999).

$$
\mathrm{CO}+\left(1+\frac{m}{2 n}\right) \mathrm{H}_{2} \rightarrow \frac{1}{n} \mathrm{C}_{n} \mathrm{H}_{m}+\mathrm{H}_{2} \mathrm{O}
$$

The main products of FT synthesis are linear n-paraffins and 1-olefins (Claeys \& van Steen, 2004). From Reaction 1.1, water is also a primary product of the FT reactions. Product selectivity in FT synthesis is temperature dependent due to the high exothermicity of the reactions occurring (Claeys \& van Steen, 2004). The low temperature FT (LTFT) synthesis conditions of $180-260^{\circ} \mathrm{C}$ and 20-30 $\operatorname{bar}(\mathrm{a})$, therefore enables syngas to be converted to long chain waxy hydrocarbons, 
with a high n-paraffin content (Espinoza et al., 1999; Leckel, 2005; Méndez et al., 2017). These waxy products can be subsequently upgraded allowing high quality, clean-burning liquid fuels to be obtained (Geerlings et al., 1999).

Commercial FT processes consist of three main sections, namely syngas production and purification, FT synthesis and product upgrading (van der Laan, 1999). The production of syngas is the most cost-intensive part of the process (Geerlings et al., 1999; van Steen et al., 2018). Consequently, the LTFT reactor should be designed to utilise syngas as efficiently as possible (Geerlings et al., 1999). In addition to the latter, to favour selectivity towards wax products, all LTFT reactors are designed to maximise heat removal (Saeidi et al., 2015). The two commercial reactor types suited to LTFT synthesis are the slurry and fixed-bed reactors. The configuration of these reactors is shown in Figure 1.1 .

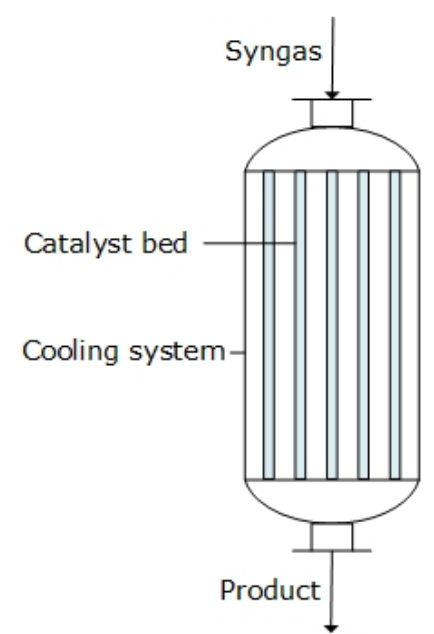

(A)

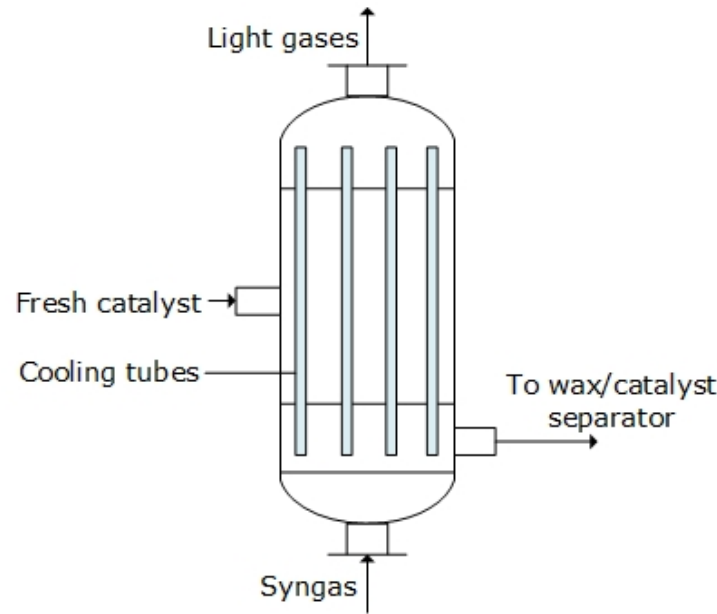

(B)

Figure 1.1: Configuration of the fixed-bed reactor $(A)$ and slurry reactor $(B)$ used for LTFT synthesis adapted from Espinoza et al. (1999)

Slurry reactors are regarded by academic and industrial researchers as the preferred reactor choice for LTFT synthesis. This opinion arises out of their ability to maintain near isothermal operation because of the presence of a significant liquid phase acting as a heat sink. Heat transfer coefficients up to $1000 \mathrm{~W} / \mathrm{m}^{2} / \mathrm{K}$ can be obtained, contributing to high liquid phase productivity and wax selectivity (Espinoza et al., 1999, Sie \& Krishna, 1999). Additional advantages include simple construction at low cost, low pressure-drop during operation and ease with which spent catalyst can be replaced (Saeidi et al., 2015). The design of a slurry reactor, however, remains in the intermediate phase because of the challenges posed by an incomplete knowledge of gas-liquid hydrodynamics, mass and heat transfer phenomena and liquid-solid suspension behaviour which are key to predicting performance (Saeidi et al., 2015). Furthermore, an important part of their operation is the separa- 
tion of wax from suspended catalyst. This requires a unique separation process. Collectively, these factors make scale-up from pilot-plant to large-scale operation challenging (Saeidi et al., 2015).

Fixed-bed reactors have a well-established design since it is similar to a tubular heat exchanger. The reaction occurs on the tube-side and a coolant, typically boiling water, passes over the tubes on the shell-side for temperature control. Despite their well-established design, fixed-bed reactors are more expensive to construct, have a higher pressure-drop during operation and their design poses challenges to catalyst loading and temperature control due to poor heat transfer. Heat transfer rates are approximately five times lower than in slurry reactors (Geerlings et al., 1999). It is, however, attractive since it operates with a concentration gradient of plug-flow, has a higher catalyst hold-up and no unique separation process is required to separate catalyst from wax (Geerlings et al., 1999; Méndez et al., 2017). This is advantageous in terms of time savings and lower operating costs. The prediction of the behaviour of a single-tube reactor is analogous to that of a large-scale multi-tubular reactor. This allows for ease of scale-up to industrial-sized operations.

A major challenge in the simulation and design of either reactor is the lack of reliable FT kinetic models (van der Laan, 1999, Claeys \& van Steen, 2004). While the kinetics of the FT reactions have been extensively studied leading to a large variety of FT rate expressions, a model developed from first principles to describe reactant consumption and product formation in a satisfactory manner has not yet been developed. The challenge to developing such a model is the complexity of the FT reaction mechanism and the large number of possible reaction products (Visconti et al., 2007).

The polymerisation character of FT synthesis means that its product distribution could ideally be described by models based on probability theory. However, significant deviations have been reported from these models, namely a high methane yield, low ethene yield and an exponential decrease in 1-olefin content favouring n-paraffin formation of the same carbon number (Claeys \& van Steen, 2004). Most comprehensive kinetic models focus on finding an effective description for these deviations. Commonly accepted explanations are the different activation energies for methane and ethene from other n-paraffins and 1-olefins and the re-adsorption of 1-olefins, favouring its secondary reaction towards n-paraffins of the same carbon number (Claeys \& van Steen, 2004; Marchese et al. 2019). The extent of these secondary reactions is often assumed to be influenced by carbon number dependent processes such as diffusion, physisorption or solubility (Claeys \& van Steen, 2004). Complex kinetic models are developed based on these assumptions, despite the reasons behind the deviations from ideal polymerisation behaviour being unknown and disputed (Puskas \& Hurlbut, 2003). Few models in literature are also extended to include high carbon numbers products (Marchese et al., 2019). 
Dictor \& Bell (1986) indicate that the reported deviations in FT synthesis from ideal polymerisation are the result of the chemistry of FT synthesis. The continued search for a kinetic explanation of these deviations ignores that FT synthesis has many aspects of an equilibrium-controlled process. The work of Norval \& Phillips (1990) and Norval (2008) has shown that the products of FT synthesis are distributed according to thermodynamics, with the yield of hydrocarbons controlled kinetically. This arises because the rates of chain growth are much faster than the rate of monomer formation from CO (Norval, 2008; Allie \& Nyathi, 2019; Hensen et al., 2020). If FT synthesis could be modelled as equilibrium-controlled process, the kinetic model could consist of simpler rate expressions. An example of a hydrocarbon system in which this strategy has successfully been applied is 1-olefin oligomerisation (Sealy, 1996; Norval, 2008).

At the operating conditions of LTFT synthesis, liquid formation is unavoidable (Mthombeni, 2009). Many authors, however, neglect the formation of liquid in their reactors and model FT synthesis as a reaction occurring in the vapour phase only (Masuku et al., 2011, 2012a). The formation of liquid plays an important role in determining the kinetics and selectivity of FT synthesis (Glasser et al., 2012). Furthermore, the successful design of slurry reactors depends on the presence of a liquid phase since its composition affects gas-liquid hydrodynamics and mass transfer, while in fixed-bed reactors the presence of a liquid phase affects heat and mass transfer and pressure-drop (Hildebrandt et al., 2010; Glasser et al., 2012; Visconti, 2014).

In heterogeneous systems, the reaction occurs close to the interphase between phases (Fogler, 1999). The effect of liquid formation has thus been effectively modelled by assuming vapour-liquid equilibrium exists (Schulz \& Claeys, 1999; van der Laan, 1999; Ahón et al., 2005; Mthombeni, 2009). However, the poor modelling of the multiphase nature of FT synthesis has meant that the effect of vapour-liquid equilibrium on the kinetic behaviour is not adequately accounted for. This is since thermodynamic and kinetic models have been treated independently. In n-paraffin hydrocracking, vapour-liquid equilibrium has been shown to play an important role in determining product selectivity (Accolla, 2006), Le Grange, 2009). These findings arise because thermodynamic and kinetic models are coupled and dependent on each other (Mthombeni, 2009). It is therefore essential to include the effect of vapour-liquid equilibrium on the kinetics of FT synthesis.

The effect of $\mathrm{CO}$ conversion on the product distribution and selectivity of FT synthesis is often not reported in literature to a great level of detail. This is since FT reactors operate at low per pass conversions with a syngas recycle (van Steen et al., 2018). With renewable feeds like biomass receiving more attention, small-scale FT plants are becoming more attractive (Tucker \& van Steen, 2019). Small-scale operation is, however, limited by economies of scale (Sie \& Krishna, 1999; Tucker \& van Steen, 2019). Thus, to be viable the FT reactor should operate at the highest possible CO conversion level. The design of a single-pass syngas conversion plant therefore 
requires a kinetic model which can predict the dependence of the selectivity of the FT process on CO conversion (van Steen et al., 2018).

The following work will focus on the development of a kinetic model for LTFT synthesis that combines the interaction between reaction equilibrium, kinetics and vapour-liquid equilibrium to explain the observed behaviour. A completely predictive model of this form will be an important tool for engineers involved in designing an FT process to maximise wax selectivity for fuel production.

\subsection{Problem Solving Methodology}

The complexity of the FT reaction system means that a systematic approach is necessary to aid in the development of a mathematical model for this reaction. This goal was subdivided into different building blocks, each of which lays the groundwork for the next phase of the model development.

It is necessary to gather as much information as possible about the FT reaction and the strategies used to model its phenomena. This begins with a literature on review on the FT reactions and catalysts, selectivity, kinetics, thermodynamics and phase behaviour in Chapter 2. The effective modelling of the $n$-paraffin and 1-olefin products of the $\mathrm{FT}$ reactions requires that the physical properties of these species are known. This is an essential prerequisite for chemical and phase equilibrium calculations and its effect on the kinetic behaviour of FT synthesis. The focus of Chapter 3 is the development of a physical property and thermodynamic database for FT synthesis.

Chapter 4 presents the development of a kinetic model for FT synthesis based on the equilibriumcontrol in the product distribution. The model aims to effectively accounts for the observed FT reaction behaviour. The work in this chapter is a novel aspect of the model. Further testing of the model as a function of process conditions ( $\mathrm{CO}$ conversion, temperature, pressure and $\mathrm{H}_{2} / \mathrm{CO}$ feed ratio) will also be carried out.

Following the model development, the relationship between the model input parameters and model output will be investigated in Chapter 5. This will provide insight regarding the reaction pathway and which model parameters can be regressed from experimental data. Thereafter, validation of the vapour phase model is performed through regression of the model rate constants from experimental data in Chapter 6.

Lastly, the effect of liquid formation on product selectivity and the product distribution of FT synthesis will be investigated in Chapter 7 . Vapour-liquid equilibrium will be included in the kinetic and reactor model with comparisons made between the prediction of the single- and two-phase simulations. The applicability of the single and two-phase simulations will be analysed through comparison to experimental data. 


\section{Chapter 2}

\section{Literature Review}

\subsection{Fischer-Tropsch Chemistry and Catalysis}

\subsubsection{Fischer-Tropsch Reactions}

Fischer-Tropsch (FT) synthesis is a polymerisation reaction which involves the catalytic hydrogenation of $\mathrm{CO}$ into a wide range of products of different carbon number and functionality (Claeys \& van Steen, 2004: Subiranas, 2009). All reaction pathways consist of monomer and initiator formation from $\mathrm{CO}$ and $\mathrm{H}_{2}$, followed by chain growth, chain termination and finally product desorption Claeys \& van Steen, 2004). Although the product distribution of FT synthesis is complex, it shows order with respect to the class and size of the products formed (Claeys and van Steen, 2004). Under the low temperature FT (LTFT) conditions of $180-260^{\circ} \mathrm{C}$ and $20-30$ bar(a), the main products are linear n-paraffins and 1-olefins, with their formation described by the overall Reactions 2.1 and 2.2 (Claeys \& van Steen, 2004; Méndez et al., 2017).

$$
\begin{gathered}
n \mathrm{CO}+(2 n+1) \mathrm{H}_{2} \rightarrow \mathrm{C}_{n} \mathrm{H}_{2 n+2}+n \mathrm{H}_{2} \mathrm{O} \\
n \mathrm{CO}+2 n \mathrm{H}_{2} \rightarrow \mathrm{C}_{n} \mathrm{H}_{2 n}+n \mathrm{H}_{2} \mathrm{O}
\end{gathered}
$$

At the conditions of LTFT synthesis, the product distribution changes from mainly 1-olefins of low carbon number to a purely n-paraffinic wax product at high carbon number (Claeys \& van Steen, 2004). From the overall Reactions, 2.1 and 2.2. water is a major product of the FT reactions. The water gas shift (WGS) Reaction 2.3 may also occur in some systems where there is significant catalyst activity or when low $\mathrm{H}_{2} / \mathrm{CO}$ ratios are present (Subiranas, 2009; Rafiq et al., 2011; Bukur et al., 2012).

$$
\mathrm{CO}+\mathrm{H}_{2} \mathrm{O} \rightleftharpoons \mathrm{H}_{2}+\mathrm{CO}_{2}
$$


Side products include oxygenated compounds, which are mainly n-alcohols, (see Reaction 2.4) and branched compounds (Claeys \& van Steen, 2004). Their formation is dependent on process conditions e.g. n-alcohol formation becomes more favourable with increasing pressure (van der Laan, 1999; Norval, 2008). This is shown by the 30-40 bar(a) pressure conditions of the Qian

et al. (2013) experiments. The degree of branching becomes more favourable with increasing temperature. As such, it is present in low amounts in LTFT synthesis, accounting for $<5 \mathrm{wt} . \%$ of the product (Claeys \& van Steen, 2004). Alcohols account for less than $10 \mathrm{wt}$ \% of the product and have been found to be less than 5 wt.\% in some studies (Qian et al., 2013).

$$
n \mathrm{CO}+2 n \mathrm{H}_{2} \rightarrow \mathrm{C}_{n} \mathrm{H}_{2 n+1} \mathrm{OH}+(n-1) \mathrm{H}_{2} \mathrm{O}
$$

Based on overall chemical equilibrium calculations of the reactions involved, methane, the least desired product, is favoured over other hydrocarbons i.e. its formation is unavoidable, the formation of $n$-paraffins is preferred relative to 1-olefins and $n$-alcohols, the least favoured 1-olefin is ethene and the least favoured $n$-alcohol is methanol under LTFT synthesis conditions (Subiranas, 2009). Product selectivity depends on process conditions, namely temperature, pressure and $\mathrm{H}_{2} / \mathrm{CO}$ feed ratio (Espinoza et al., 1999), the reactor system and the catalyst, its formulation and physical properties (Claeys \& van Steen, 2004).

\subsubsection{Fischer-Tropsch Catalysts}

A common property of the metals used as FT catalysts is that they are all active for hydrogenation reactions (Pichler \& Schulz, 1970). The metals ruthenium (Ru), cobalt (Co), iron (Fe) and nickel $(\mathrm{Ni})$ are the active components of FT catalysts. Ni has a high methane selectivity. This makes it a more effective methanation catalyst (Subiranas, 2009). Ru is the most active with the highest wax selectivity (van der Laan, 1999; Subiranas, 2009). Ru-based catalysts are, however, not used commercially because of the limited world reserves and high cost (Subiranas, 2009; Saeidi et al., 2015). Fe- and Co-based catalysts remain the only viable options used commercially (Saeidi et al., 2015).

The most active Fe-based catalysts are precipitated catalysts, while the most active Co-based catalysts are supported catalysts (Espinoza et al., 1999; Subiranas, 2009). This preparation method results in a higher site density and consequently a higher intrinsic activity for Fe-based catalysts (Espinoza et al., 1999). This advantage of precipitated Fe-based catalysts over supported Co-catalysts decreases and disappears as syngas conversion and hence water formation increases (Espinoza et al., 1999; Subiranas, 2009; Saeidi et al., 2015). Water acts as an inhibitor on the surface of an Febased catalyst. This means the catalyst is (re)oxidised at lower water partial pressures relative to 


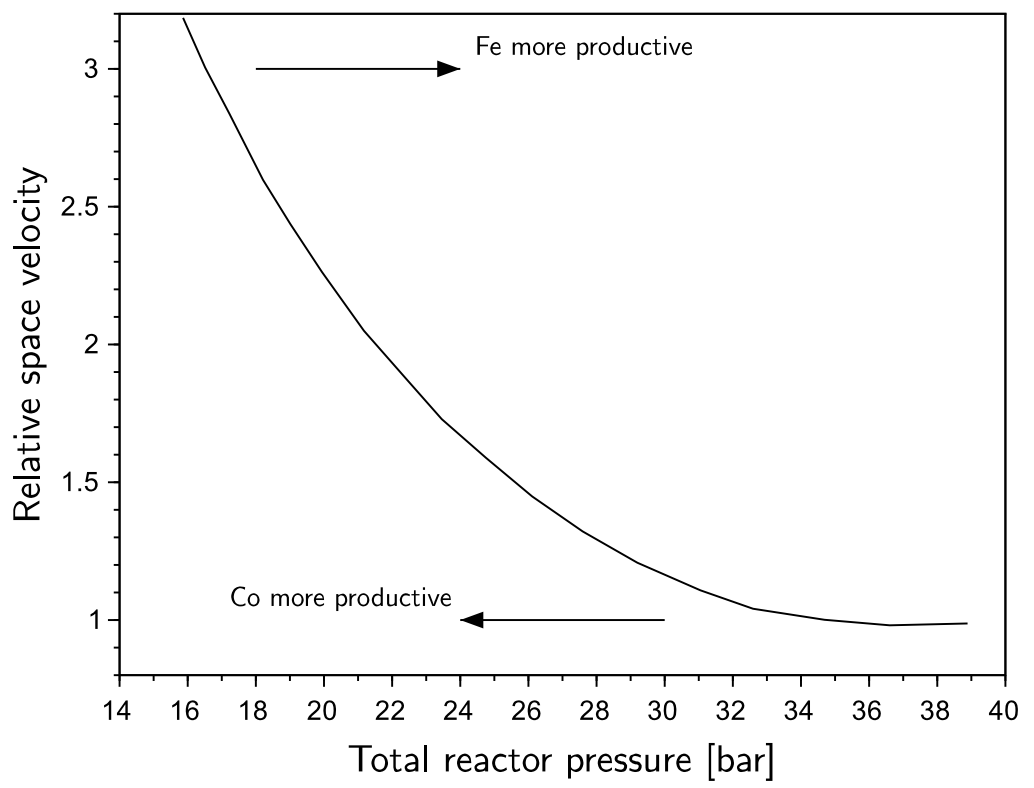

Figure 2.1: Productivity comparison between a commercial Sasol precipitated Fe catalyst $\left(240^{\circ} \mathrm{C}\right)$ and a commercial Sasol supported Co catalyst $\left(220^{\circ} \mathrm{C}\right)$ adapted from Espinoza et al. (1999)

Co-based catalysts. Consequently, there are fewer free active sites as reaction occurs (Saeidi et al., 2015). The result of this behaviour is that there are distinct operating regimes in which either Feor Co-based catalysts may be more productive towards wax. From Figure 2.1. Fe-based catalysts are more productive at higher space velocity (lower water partial pressure) and operating pressure and vice-versa for Co-based catalysts (Espinoza et al., 1999). This means Co-based catalysts are more productive under higher per pass syngas conversion regimes.

It is known that a Co active site has a higher turnover number relative to an Fe active site (Espinoza et al., 1999). This allows for not just operation at lower pressure but also lower temperatures using Co-based catalysts (Visconti, 2014). Product selectivity is a strong function of temperature. Typical operating temperatures in fixed-bed reactors are $200-230{ }^{\circ} \mathrm{C}$ for Co-based catalysts and $220-250^{\circ} \mathrm{C}$ for Fe-based catalysts (Jess \& Kern, 2009). This results in Fe-based catalysts having a higher 1-olefin selectivity (dehydrogenation more favourable) and due to its greater productivity at higher pressure from Figure 2.1, also a higher oxygenate selectivity (Teng et al., 2006). It is reported that oxygenated compounds account for $6-12 \mathrm{wt} . \%$ of the product in commercial Fe-based LTFT processes (Claeys \& van Steen, 2004).

The lower operating temperature when using Co-based catalysts results in the production of mainly linear n-paraffins (hydrogenation more favourable). While more methane is produced than on Fe- 
based catalysts, this is offset by the lower operating temperature leading to greater wax selectivity (van Steen et al., 2018). Co-based catalysts also have a lower WGS activity relative to Fe-based catalysts (Patzlaff et al., 1999; Visconti et al., 2007; Visconti, 2014). This ensures high thermal efficiency, more effective carbon utilisation as most oxygen atoms in $\mathrm{CO}$ react to form water and a high yield of FT products (Visconti, 2014; van Steen et al., 2018). $\mathrm{CO}_{2}$ selectivity is typically below $1 \mathrm{~mol} . \%$, provided the $\mathrm{H}_{2} / \mathrm{CO}$ ratio is approximately 2. Although Co metal is more expensive relative to Fe metal, the longer catalyst lifetime and ability to later recover the spent metal offsets the cost (van der Laan, 1999; Visconti, 2014). Together with the higher productivity at a higher per pass syngas conversion and greater wax selectivity, it is best suited to LTFT synthesis.

\subsubsection{Fischer-Tropsch Reaction Mechanism}

The mechanistic details of the FT reactions remains controversial due to the wide variety of products. The differences between proposed mechanisms arise from different assumptions regarding the form of the monomer and initiator and the steps leading to their formation (Mousavi et al., 2015).

There is, however, agreement that it is a chain polymerisation reaction ${ }^{1}$ (Sie et al., 1991; van der Laan, 1999; Lozano-Blanco et al., 2006). According to van der Laan (1999), the mechanism should consist of the following steps:

1. $\mathrm{CO}$ and $\mathrm{H}_{2}$ adsorption and dissociation.

2. Monomer and chain initiator formation.

3. Chain growth.

4. Chain termination and product desorption.

5. Re-adsorption and further reaction.

The mechanistic situation can be simplified by considering only a subset of the products. Under LTFT conditions, n-paraffins and 1-olefins are the main products. For their formation, the carbide mechanism is most accepted (Patzlaff et al., 1999, van der Laan, 1999; Mousavi et al., 2015). Here, carbide or $\mathrm{CH}_{2}$ is considered as the monomer. While only Fe-based catalysts form stable carbide phases, elements of this mechanism were also observed on Co- and Ru-based catalysts (Mousavi et al., 2015). $\mathrm{CH}_{2}$ cannot polymerise alone as the primary product would be ethene (Claeys \& van Steen, 2004). This emphasises the need for a chain initiator. This led to carbide mechanism modifications, with chain growth occurring through alkyl insertion i.e. the alkyl mechanism. Here,

\footnotetext{
${ }^{1}$ Growth of the hydrocarbon chain occurs through the addition of a single $C_{1}$ monomer unit at the end of the existing chain.
} 
$\mathrm{CH}_{2}$ and $\mathrm{CH}_{3}$ are the monomer and initiator respectively. This highlights the importance of $\mathrm{H}_{2}$ as in its presence, $\mathrm{CH}_{2}$ incorporates into long chain hydrocarbons (Mousavi et al., 2015).

\section{Initiation:}

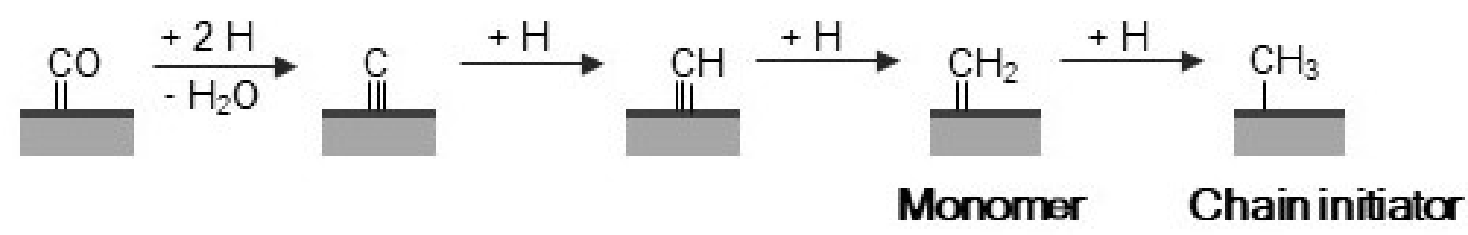

\section{Chaingrowth:}

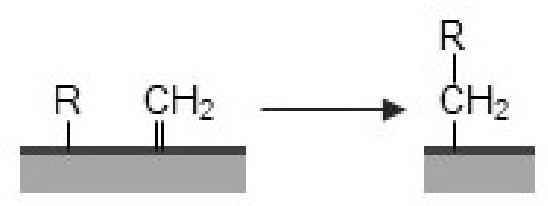

\section{Termination/desorption:}

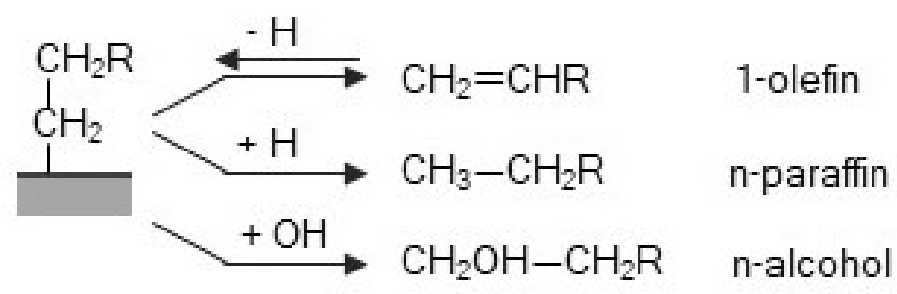

Figure 2.2: Initiation, chain growth and termination steps in the alkyl mechanism adapted from Claeys \& van Steen (2004)

The initiation, chain growth and termination steps in the alkyl mechanism are summarised in Figure 2.2. Chain growth occurs through the addition of $\mathrm{CH}_{2}$ monomers. Product termination occurs through either $\beta$-dehydrogenation or hydrogenation to a 1-olefin or $n$-paraffin respectively. This mechanism works well for $\mathrm{n}$-paraffin and 1-olefin formation but it cannot explain the formation of oxygenates (Claeys \& van Steen, 2004; Förtsch et al., 2015; Mousavi et al., 2015). From Figure 2.2. it has been proposed that the coupling of a surface hydroxyl $(\mathrm{OH})$ species and an alkyl group may lead to n-alcohol formation. There is, however, a lack of experimental proof for this proposal (Claeys \& van Steen, 2004). For systems with more oxygenated products, it might be necessary to use the CO insertion mechanism to explain the formation of all FT products (Qian et al., 2013; Todic et al., 2014). While this mechanism is based on analogy from homogeneous catalysis with a lack of experimental proof, it is regarded as the main route leading to the formation of oxygenates 
(Claeys \& van Steen, 2004; Mousavi et al., 2015).

The combination of different mechanisms could allow for all FT products to be accounted for e.g. a CO insertion-alkyl mechanism (Patzlaff et al., 1999; Mousavi et al., 2015). Generally, the formation of oxygenates is small in comparison to the main products (Wang et al., 2003b; Teng et al., 2006; Visconti et al., 2007). This suggests that reaction along the CO insertion pathway may be negligible in comparison to the alkyl mechanism pathway (Kwack et al., 2011). As such, a single mechanism can be considered, with most kinetic modelling studies using the alkyl mechanism. From Figure 2.2, it has been observed that the desorption of 1-olefins is reversible which leads to the occurrence of secondary reactions (van der Laan, 1999; Claeys \& van Steen, 2004). The importance of secondary reactions and their effect on the type and molecular weight of the hydrocarbon products as well as the selectivity character of FT synthesis will be discussed later.

\subsubsection{Product Selectivity}

The agreement about the polymerisation character of FT synthesis and that the chain growth steps occur through the addition of a $C_{1}$ monomer means that the nature of the product distribution can be estimated using probability theory. This was first developed and applied by Flory for free radical step growth polymerisation (Fogler, 1999; Förtsch et al., 2015). It was extended and applied to FT synthesis by Anderson and Schulz (Davis, 1992; van der Laan, 1999; Puskas \& Hurlbut, 2003). It was recognised that the distribution of n-paraffins could be described by Equation 2.5, the ASF distribution ${ }^{2}$

$$
w_{n}=n(1-\alpha)^{2} \alpha^{n-1}
$$

In Equation 2.5, $n$ refers to the number of carbon atoms in the product, $w_{n}$ is the weight fraction of the product containing $n$ carbon atoms and $\alpha$ is the chain growth probability. The latter is defined as the rate of propagation $r_{p}$ relative to the sum of the propagation and termination rates $r_{t}$ in Equation 2.6 .

$$
\alpha=\frac{r_{p}}{r_{p}+r_{t}}
$$

A key assumption of the ASF distribution is that $\alpha$ is independent of carbon number. This allows the entire product distribution to be characterised by a single parameter, $\alpha$, for values between zero and one (Subiranas, 2009, Förtsch et al., 2015). This is shown in Figure 2.3. FT product selectivity can therefore be described in a lightweight, tractable manner that is easy to understand because the value of $\alpha$ can easily be extracted from experimental/plant product distributions (Subiranas, 2009; Förtsch et al., 2015). While the ASF distribution does not distinguish between product types, it

\footnotetext{
${ }^{2}$ To describe the mole fraction of products containing $n$ carbon atoms, $y_{n}=(1-\alpha) \alpha^{n-1}$ is used.
} 
can be applied separately to different product homologous series $3^{3}$

From Figure 2.3, there is a shift from mainly low molecular weight products at low $\alpha$ to high molecular weight products at high $\alpha$. This is expected for an exothermic reaction i.e. lower temperatures favour a product of higher molecular weight (Claeys \& van Steen, 2004). This is consistent with the definition of $\alpha$ in Equation 2.6. For $\alpha \approx 0.62$, the FT product is a mixture of n-paraffins, 1-olefins and oxygenates, while for $\alpha \approx 0.82$, the only significant product corresponds to $n$-paraffins (Davis, 1992). Reports on Co-based catalysts indicate $\alpha$ values between $0.85-0.95$ (Sie, 1998). For their LTFT processes targeting wax production, Sasol report $\alpha \approx 0.94-0.95$ using Co- and Fe-based catalysts respectively (Espinoza et al., 1999). It can therefore be inferred that the wax product is mainly paraffinic in nature.

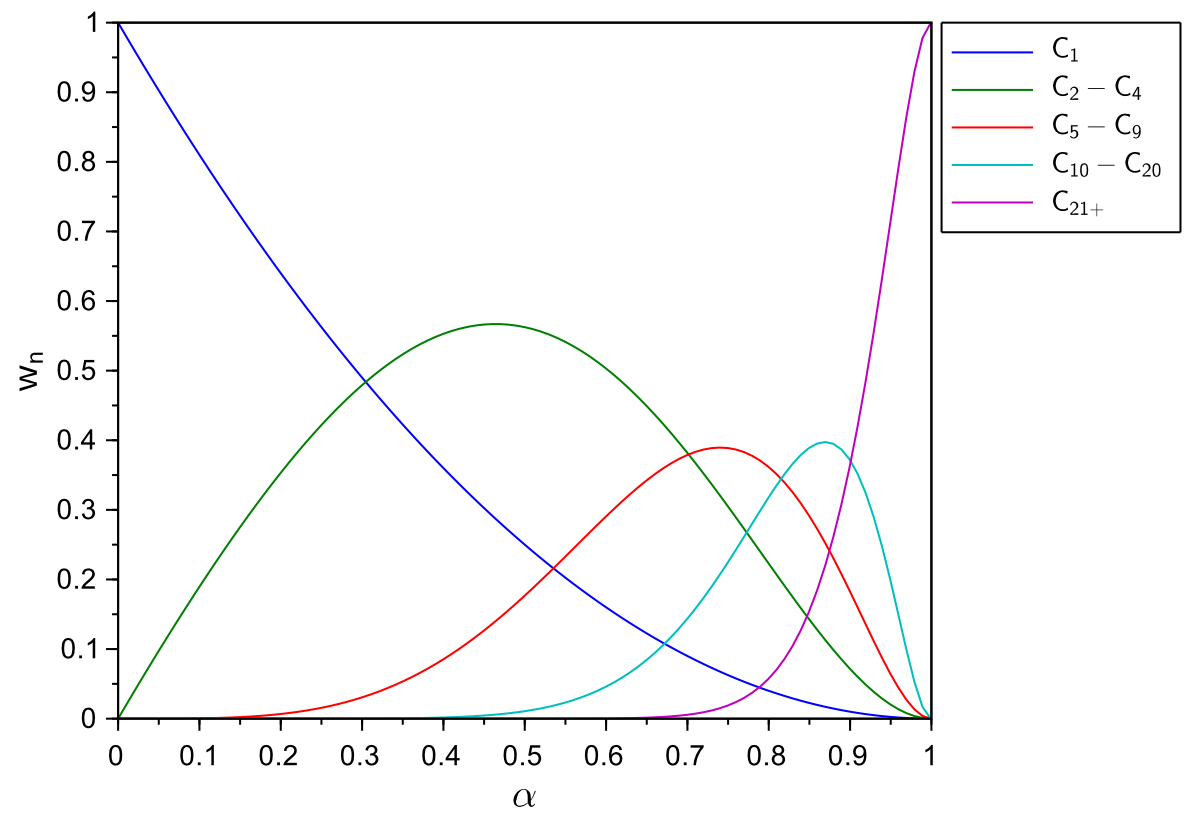

Figure 2.3: Weight fraction of the total hydrocarbon distribution as a function of $\alpha$ using Equation 2.5

In practise, the ideal ASF distribution shown in Figure 2.3, is not obtained, particularly in LTFT processes. The majority of reported ASF distributions are only applicable in the $\mathrm{C}_{4}-\mathrm{C}_{12}$ region. This made the experimental determination of $\alpha$ arbitrary (Puskas \& Hurlbut, 2003). The products of the FT reactions show the following characteristics based on carbon number (van der Laan, 1999: Claeys \& van Steen, 2004):

\footnotetext{
${ }^{3} \mathrm{~A}$ homologous series is a family of compounds which have the same functional group and similar chemical properties.
} 
1. The highest concentration in the carbon number distribution is observed at $C_{1}$. There is a monotonic decrease in concentration with carbon number. A local maximum is observed at $\mathrm{C}_{3} / \mathrm{C}_{4}$.

2. The concentration of and selectivity to ethene is low in comparison to other 1-olefins.

3. There is an asymptotic decrease in 1-olefin concentration to zero. This is observed as an exponential decrease in the olefin-paraffin ratio (OPR) for $n \geq 3$.

4. When fitting experimental product distribution data to the ASF distribution, a 'change' in $\alpha$ is observed for linear n-paraffins only and not 1-olefins.

5. The yield of $n$-alcohols is at a maximum at $C_{2}$ and decreases with carbon number. The low methanol yield is the result of thermodynamic limitations.

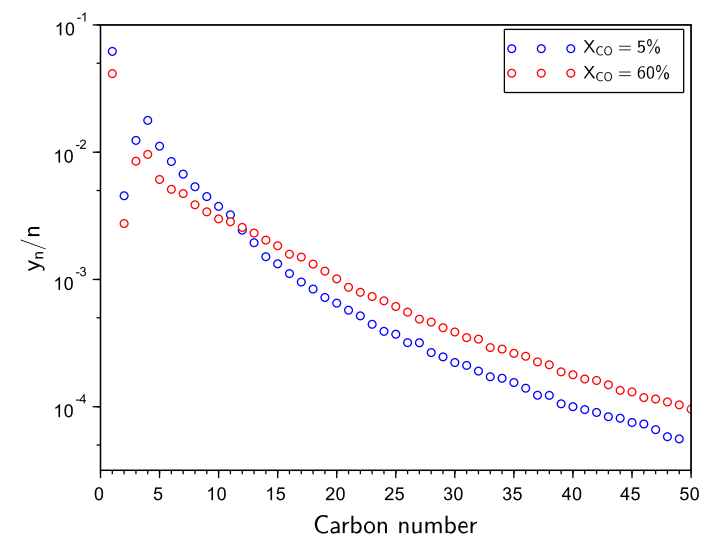

(a) Product distribution

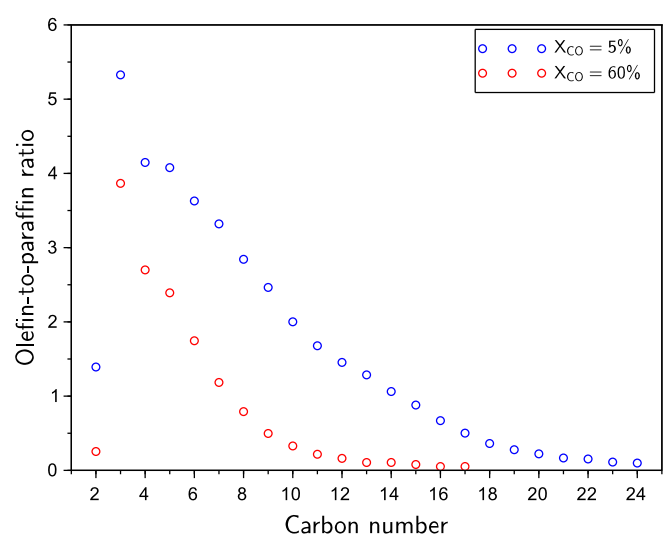

(b) Olefin-to-paraffin ratio (OPR)

Figure 2.4: Molar product distribution and olefin-to-paraffin ratio as a function of carbon number in LTFT synthesis. Data from Iglesia et al. (1993) at $203^{\circ} \mathrm{C}, 6 \mathrm{bar}(\mathrm{a})$ and $\mathrm{H}_{2} / \mathrm{CO}$ feed ratio of 2.1 for a $\mathrm{Ru} / \mathrm{TiO}_{2}$ catalyst.

The cause of these deviations from the ASF distribution is the result of the chemistry of FT synthesis (Dictor \& Bell, 1986). In LTFT synthesis, the main deviations are a high methane yield, low ethene yield and the 'change' in $\alpha$ observed for n-paraffins. These features are shown in Figure 2.4. 


\subsubsection{Possible Causes for Deviations from the ASF Distribution}

\subsubsection{High Methane Yield}

It was originally believed that mass and heat transfer limitations caused the high methane yield. However, its occurrence was still observed in experiments without these transport phenomena (van der Laan, 1999). Active sites on the surface of FT catalysts may result in a site that favours the methanation reaction relative to chain growth (Claeys \& van Steen, 2004). This indicates that the mechanism for methane formation potentially differs from that for other $\mathrm{n}$ paraffins, where the activation barrier may be lower. This is consistent with methane being the most thermodynamically favoured product. It is also possible that there are several possible routes leading to methane formation (Patzlaff et al., 1999). Possible routes of methane formation include direct hydrogenation of a methyl surface species $\left(\mathrm{CH}_{3}\right)$, direct hydrogenation of a $\mathrm{CH}_{2}$ monomer and possible hydrogenolysis ${ }^{4}$ to methane, although this is strongly inhibited by $\mathrm{CO}$. While a reduction in the rate of formation of methane is not possible, it can be kinetically controlled using temperature and pressure conditions. This enables the selectivity to wax to be maximised relative to that of methane (Claeys \& van Steen, 2004, van Steen et al., 2018).

\subsubsection{Low Ethene Yield and Change in $\alpha$}

At around $\mathrm{C}_{10}$, a change in $\alpha$ is observed in the ASF distribution for $n$-paraffins, but not for 1olefins. This is shown in Figure 2.4. This represents an exponential decrease in the olefin-to-paraffin ratio (OPR) for $n \geq 3$ i.e. the content of a 1-olefin of carbon number $n$ decreases asymptotically to zero, with the formation of the corresponding $n$-paraffin of the same carbon number being favoured. From Figure 2.5, this indicates that $\alpha$ is not independent of carbon number, with increases observed to a maximum value (van der Laan, 1999). This represents enhanced chain growth.

There is qualitative agreement that the occurrence of secondary reactions of 1-olefins causes these phenomena (Claeys \& van Steen, 2004). Secondary reactions occur due to interactions of the $\pi$-bond of 1-olefins with the catalyst surface, resulting in the reversibility of their desorption step in Figure 2.2. This leads to further reactions which are indistinguishable from the reactions of primary formed 1-olefins (Iglesia et al., 1991; Claeys \& van Steen, 2004). These reactions are consecutive in nature and are supported by varying residence time and 1-olefin co-feeding studies. The extent of secondary reactions increases in the order Fe, Ru and Co (van der Laan, 1999). This highlights the suitability of Fe for producing 1-olefins and that high n-paraffin yields are obtained using Co. In Equation 2.7, the major 1-olefin reactions are given in order of increasing extent (Claeys \& van

\footnotetext{
${ }^{4}$ Hydrogenolysis is a chemical reaction in which carbon-carbon or carbon-heteroatom bonds are broken by hydrogen.
} 
Steen, 2004).

$$
\text { reinsertion } \gg \text { isomerisation }>\text { hydrogenation }
$$

Equation 2.7 indicates that secondary hydrogenation is the least important secondary reaction. This was deduced based on observations that the selectivity of paraffins of low carbon number remained independent of residence time. Secondary hydrogenation is believed to be strongly inhibited by CO, the most abundant adsorbed species (Iglesia et al., 1993; Todic et al., 2014). Secondary hydrogenation may occur depending on the catalyst ( $\mathrm{Co}$ rather than $\mathrm{Fe}$ ) and process conditions (high $\mathrm{H}_{2}$ partial pressures) (van der Laan, 1999). Unlike secondary hydrogenation, reinsertion and isomerisation are not inhibited by $\mathrm{CO}$. At high $\mathrm{CO}$ and water partial pressures, reinsertion is believed to be the most important secondary reaction causing the observed deviations from the ASF distribution (Claeys \& van Steen, 2004).

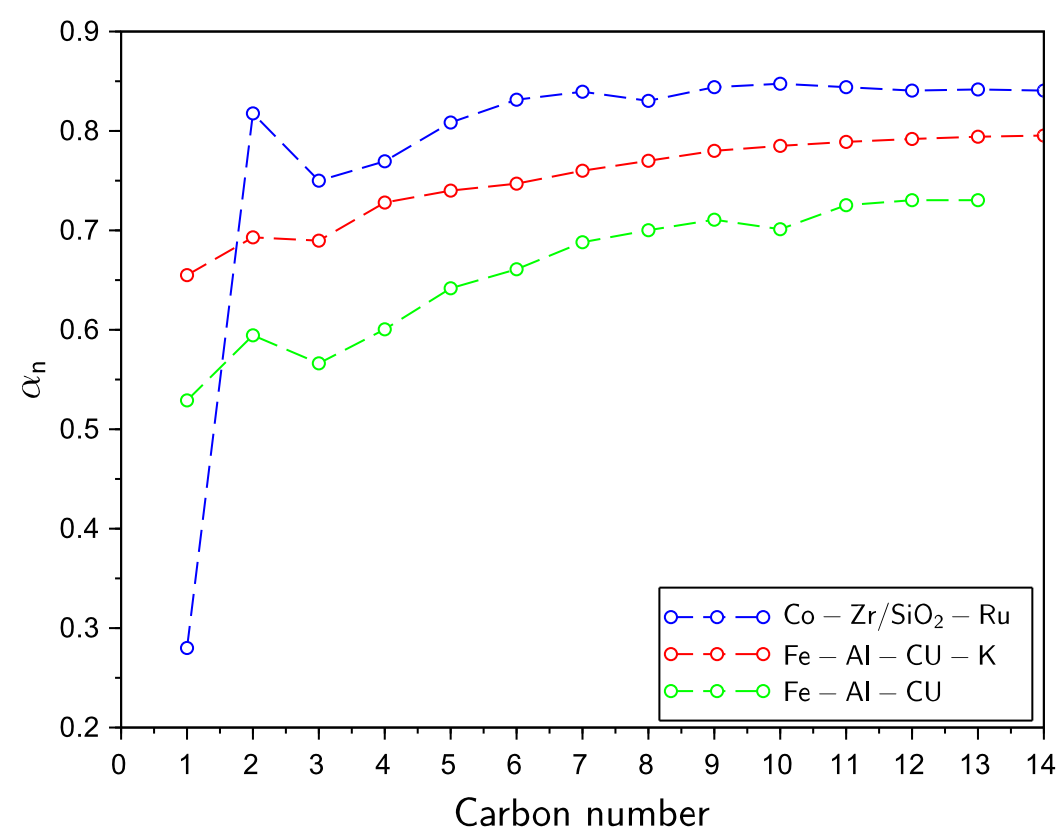

Figure 2.5: Chain growth probability as a function of carbon number for different catalysts adapted from Schulz \& Claeys (1999)

The low ethene yield in comparison to other 1-olefins is attributed to its $\approx 10-25$ times greater reactivity for reinsertion relative to other 1-olefins (van der Laan, 1999; Claeys \& van Steen, 2004). This favours its involvement in secondary reaction like reinsertion, where it may act as a chain starter. This is consistent with it being the least thermodynamically favoured 1-olefin. Mthombeni (2009) indicated that secondary reactions alone cannot cause the observed deviations from the ASF distribution. It is rather the combination of secondary reactions and carbon number 
dependent processes. This combined effect causes the increase in $\alpha$ at a given carbon number observed in Figure 2.5, favouring the formation of $n$-paraffins and the decrease in 1-olefin content (Claeys \& van Steen, 2004). The possible carbon number dependent processes for 1 -olefins that are involved will be discussed in the Kinetic Modelling section.

\subsection{Kinetic Modelling}

To perform FT reactor design, there is a need to describe both reactant consumption and product formation. Although there are different approaches that have been applied in FT kinetic modelling, two main approaches can be distinguished (van der Laan, 1999; Visconti et al., 2007; Förtsch et al., 2015: Hillestad et al., 2016):

1. Empirical or Langmuir-Hinshelwood (LH) based rate expressions to describe the FT reaction rate per mole of $\mathrm{CO}$. This is paired with a selectivity model like the ASF distribution to describe product formation.

2. Mechanistic-based models which describe reactant consumption and product formation simultaneously. This requires a reliable mechanism, with LH theory used to describe each reaction step of adsorption, surface reaction and desorption.

\subsubsection{Overall Fischer-Tropsch Reaction Rate and Selectivity Models}

The description of the overall FT reaction rate is a minimalistic approach to kinetic modelling. It provides a good compromise between chemical description and mathematical tractability and a quick indication of the system behaviour (Rafiq et al., 2011). The focus of this approach involves describing the rate of consumption of the reactants, $\mathrm{H}_{2}$ and $\mathrm{CO}$ (Förtsch et al., 2015). FT reaction rate expressions can be formulated empirically or derived using LH theory. The latter is more common, in which a simple mechanism is proposed leading to monomer formation (Claeys \& van Steen, 2004). It is often assumed that the rate of CO consumption is the rate-determining sten ${ }^{5}$ as this controls the average chain length (Claeys \& van Steen, 2004).

This only describes reactant consumption. To describe product formation and selectivity, models describing the overall FT reaction rate need to be paired with a product distribution model like the ASF distribution in Equation 2.5 (Wang et al., 2003b). Equation 2.5 results in hydrocarbons being lumped based on carbon number. The rate of formation of a hydrocarbon of a certain carbon number relies on $\alpha$, which is determined empirically.

\footnotetext{
${ }^{5}$ The slowest step that is involved in product formation in a reaction mechanism.
} 
Philippe et al. (2009) used carbon number lumping and the ASF product distribution to model the FT reaction kinetics. The overall FT reaction rate was determined and modified to yield separated rates of formation for $C_{1}$ and $C_{2}$. This accounted for their anomalies. For carbon numbers $n \geq 3$, the rate of formation of carbon number $n$ is related recursively to the rate of formation of carbon number $n-1$ through $\alpha$ in Equation 2.8. Their work included products of carbon number up to $C_{50}$. Rafiq et al. (2011) followed a similar approach to Philippe et al. (2009), but lumped products into $C_{1}, C_{2}, C_{3}, C_{4}$ and $C_{5+}$ groups and $C_{2}$. Like Philippe et al. (2009), Equation 2.8 was used to describe the rate of formation of carbon numbers $n \geq 3$.

$$
r_{C_{n}}=\alpha r_{C_{n-1}}
$$

To account for deviations from the ASF distribution, a modified ASF distribution could be used, which accounts for the observed occurrences in the real distribution (Förtsch et al., 2015). Further distinction between n-paraffins and 1-olefins can be achieved using a description of the OPR e.g. the model of van der Laan (1999). It should be emphasised that Equation 2.5 and its extended analogue models are applied because they allow simple and direct extraction of the relevant parameters from experimental data i.e. $\alpha$ (Förtsch et al., 2015). A major shortcoming of using Equation 2.5 or an extension thereof is that it results in an empirical, rather than a fundamental description of FT reaction behaviour. Their applicability is therefore constrained to the experimental data used to develop the model i.e. extrapolation is not valid (Visconti, 2014). While extended models have lead to an improved description of the kinetic occurrences in FT synthesis, there are still discrepancies between model predictions and experimental data (Schulz \& Claeys, 1999). Information regarding overall syngas conversion is oversimplified and product lumping is not detailed enough for selectivity control (Wang et al., 2003a; Kwack et al., 2011). An approach from first principles is therefore required to described reactant consumption and product formation.

\subsubsection{Fischer-Tropsch Mechanistic Kinetic Models}

The description of FT reaction kinetics from first principles should be preferred for process modelling and design. This will allow for a prediction of the product distribution to be made and provide a suitable basis from which the evaluation of FT reactors can be performed (Förtsch et al., 2015). The development of rate expressions from first principles should be based on a defined mechanism for hydrocarbon formation (van der Laan, 1999; Visconti et al., 2007). For n-paraffins and 1-olefins, the main products of FT synthesis, the alkyl mechanism is most plausible and the most commonly used formulation (Marchese et al. 2019). LH theory is used to describe each elementary reaction step of adsorption, surface reaction and desorption separately. These reactions are summarised in Table 2.1 where $S$ represents an active catalyst site. 
Table 2.1: Proposed reactions in the alkyl mechanism adapted from van der Laan (1999), Visconti et al. (2007) and Visconti et al. (2011)

\begin{tabular}{lc}
\hline Reaction type & Chemical reaction \\
\hline 1. $\mathrm{H}_{2}$ dissociative adsorption & $\mathrm{H}_{2}+2 \mathrm{~S} \rightleftharpoons 2 \mathrm{H} \cdot \mathrm{S}$ \\
2. CO adsorption & $\mathrm{CO}+\mathrm{S} \rightleftharpoons \mathrm{CO} \cdot \mathrm{S}$ \\
3. CO dissociation & $\mathrm{CO} \cdot \mathrm{S} \rightleftharpoons \mathrm{C} \cdot \mathrm{S}+\mathrm{O} \cdot \mathrm{S}$ \\
\hline 4a. Water formation & $\mathrm{O} \cdot \mathrm{S}+\mathrm{H} \cdot \mathrm{S} \rightarrow \mathrm{OH} \cdot \mathrm{S}+\mathrm{S}$ \\
4b. & $\mathrm{OH} \cdot \mathrm{S}+\mathrm{H} \cdot \mathrm{S} \rightarrow \mathrm{H}_{2} \mathrm{O}+2 \mathrm{~S}$ \\
\hline 5. $\mathrm{CO}_{2}$ formation & $\mathrm{CO} \cdot \mathrm{S}+\mathrm{O} \cdot \mathrm{S} \rightarrow \mathrm{CO}_{2}+2 \mathrm{~S}$ \\
\hline 6a. Chain initiation & $\mathrm{C} \cdot \mathrm{S}+\mathrm{H} \cdot \mathrm{S} \rightleftharpoons \mathrm{CH} \cdot \mathrm{S}+\mathrm{S}$ \\
6b. & $\mathrm{CH} \cdot \mathrm{S}+\mathrm{H} \cdot \mathrm{S} \rightleftharpoons \mathrm{CH} \cdot \mathrm{S}+\mathrm{S}$ \\
6c. & $\mathrm{CH}_{2} \cdot \mathrm{S}+\mathrm{H} \cdot \mathrm{S} \rightleftharpoons \mathrm{CH}_{3} \cdot \mathrm{S}+\mathrm{S}$ \\
\hline 7. Methanation & $\mathrm{CH}_{3} \cdot \mathrm{S}+\mathrm{H} \cdot \mathrm{S} \rightarrow \mathrm{CH}_{4}+2 \mathrm{~S}$ \\
8. Chain growth & $\mathrm{CH}_{2} \cdot \mathrm{S}+\mathrm{C}_{n} \mathrm{H}_{2 n+1} \cdot \mathrm{S} \rightarrow \mathrm{C}_{n+1} \mathrm{H}_{2 n+3} \cdot \mathrm{S}+\mathrm{S}$ \\
9. Hydrogenation to n-paraffin $(n \geq 2)$ & $\mathrm{C}_{n} \mathrm{H}_{2 n+1} \cdot \mathrm{S}+\mathrm{H} \cdot \mathrm{S} \rightarrow \mathrm{C}_{n} \mathrm{H}_{2 n+2}+2 \mathrm{~S}$ \\
10. $\beta$-dehydrogenation to 1-olefin $(n \geq 2)$ & $\mathrm{C}_{n} \mathrm{H}_{2 n+1} \cdot \mathrm{S} \rightleftharpoons \mathrm{C}_{n} \mathrm{H}_{2 n}+\mathrm{H} \cdot \mathrm{S}$ \\
\hline
\end{tabular}

From Table 2.1. many elementary reactions are involved in the consumption of $\mathrm{CO}$ and $\mathrm{H}_{2}$ to final products. It is assumed that the rate of each reaction can be represented by an elementary rate expression. Rate-determining steps are assumed in the mechanism while other steps are assumed to be fast and at pseudo-equilibrium. This allows all coverage dependent terms to be eliminated from the rate expressions (Fogler, 1999). These assumptions allow for the derivation of separate rate expressions for each n-paraffin and 1-olefin in the form of Equation 2.9. In most studies, partial pressure is used as the kinetic driving force.

$$
r_{C_{n}}=\frac{(\text { kinetic term })(\text { driving force })}{(\text { adsorption term })^{p}}
$$

Since FT synthesis is a polymerisation reaction, the rate-determining step should be based on polymerisation principles i.e. initiation, chain growth and termination (Claeys \& van Steen, 2004). The termination or product formation step is often considered to be rate-determining since chain growth is desired (see Equation 2.6). The high methane yield and low ethene yield are treated as separate rate-determining steps to account for their anomalies (Wang et al., 2003b; Qian et al., 2013). Common assumptions made in the derivation of these expressions are (Qian et al., 2013):

1. Adsorbed $\mathrm{CO}$ and $\mathrm{H}_{2}$ concentrations are in equilibrium with their fluid phase concentrations.

2. N-paraffins and 1-olefins form on the same active sites.

3. Reactive intermediates have a short lifetime and are in pseudo-steady state 6

\footnotetext{
${ }^{6}$ The assumption of pseudo-steady state implies that an intermediate is so reactive that its net rate of generation is approximately zero (Fogler, 1999).
} 
4. The rate constants for hydrocarbon formation are independent of carbon number i.e. they all follow the same mechanistic pathway. The exceptions to this are methane and ethene.

5. 1-Olefin termination/desorption is reversible to account for their involvement in secondary reactions.

This approach is limited in that it requires an assumption to be made regarding the rate-determining step and the polymerisation character of the FT reaction could be lost. This could lead to the relative contribution of each step not being captured in its entirety (Allie \& Nyathi, 2019). An alternative is to avoid proposing a rate-determining step, allowing the contribution of each step to be reflected in the overall rate. This was demonstrated in the work of Visconti et al. (2007) and Visconti et al. (2011) and applied in the work of Mthombeni (2009) and Khazali (2018). The form of the rate expression for reactant consumption and product formation is implicit. Retention of the polymerisation character of the reaction means that many reactions need to be considered. Either of these approaches represent viable options to model FT reaction behaviour. The difference between most mechanistic models relates to their treatment of the carbon number dependent processes enhancing the extent of 1-olefin secondary reactions.

\subsubsection{Modelling Carbon Number Dependent Processes affecting Secondary Reac- tions}

The main secondary reaction of 1-olefins from Equation 2.7 is reinsertion. It is included in mechanistic models by making the 1-olefin desorption step reversible. The re-adsorption of 1-olefins is believed to lead to the enhanced chain growth behaviour in Figure 2.5. It is clear that it indistinguishable from the primary forming 1-olefin reactions (Iglesia et al., 1991; Claeys \& van Steen, 2004). Mthombeni (2009) indicated that this alone is not sufficient to account for deviations from the ASF distribution. In fact, modelling studies including only re-adsorption were not able to account for the exponential decrease of the OPR with carbon number e.g. Wang et al. (2003b), Teng et al. (2006) and Qian et al. (2013). It is therefore necessary to include 1-olefin carbon number dependent processes which favour re-adsorption with increasing carbon number. This introduces a carbon number dependence into the rate expressions of the model. It is important to acknowledge that a liquid phase will exist in the reactor due to the condensation of high molecular weight products (Sie \& Krishna, 1999; Wang et al., 2003a; Glasser et al., 2012). This fills the pores of the catalyst, surrounds catalyst particles and leaves with the vapour product (Satterfield et al., 1985). The formation of a liquid phase influences 1-olefin re-adsorption. With this in mind, the following 1-olefin carbon number dependent processes are believed to be involved (van der Laan, 1999, Claeys \& van Steen, 2004): 
1. Carbon number dependent diffusion in the liquid-filled pores.

2. Carbon number dependent physisorption.

3. Carbon number dependent solubility in the liquid-filled pores.

An informative analysis of these processes is provided in van der Laan (1999) and Claeys \& van Steen (2004). The main points are reproduced here.

\subsubsection{1-Olefin Carbon Number Dependent Diffusion}

The slow removal of reactive 1-olefins is believed to be caused by liquid-filled catalyst pores (Iglesia, 1993). The decrease in diffusivity with increasing carbon number results in an increase in residence time of long chain 1-olefins (Iglesia et al., 1993). Consequently, this favours the secondary conversion of a 1-olefin to the corresponding n-paraffin of the same carbon number. Iglesia et al. (1993) modelled the enhanced effect caused by diffusion on 1-olefin re-adsorption. Liquid phase fugacity was used as the kinetic driving force. All deviations from the ideal ASF distribution were accounted for, despite of an underestimation of the OPR (van der Laan, 1999). However, Kuipers et al. (1995) measured the OPR on a Co-based catalyst foil in an experiment without diffusion limitations and still observed an exponential decrease in OPR with carbon number. This suggested that diffusion limitations alone cannot account for the observed carbon number dependence and that physisorption and solubility are also involved (van der Laan, 1999). Both vapour phase physisorption and solubility have a much stronger carbon number dependence than diffusion. As such, their effect on the extent of secondary reactions would dominate (Claeys \& van Steen, 2004). Most studies therefore focus on including enhanced 1-olefin re-adsorption through carbon number dependent physisorption and solubility as summarised in Table 2.2.

Table 2.2: Strategies used by literature kinetic models to incorporate carbon number (CN) dependent processes

\begin{tabular}{|c|c|c|c|}
\hline Author & CN dependence & CN range & Driving force \\
\hline Visconti et al. (2007) & Solubility & $1-49$ & Partial pressure \\
\hline Kwack et al. (2011) & Solubility & $1-40$ & Partial pressure \\
\hline Visconti et al. $(2011)$ & Solubility & $1-49$ & $\begin{array}{l}\text { Liquid phase mole } \\
\text { fraction }\end{array}$ \\
\hline Todic et al. (2014) & Physisorption & $1-15$ & Partial pressure \\
\hline Sage et al. (2014) & Physisorption & $1-15$ & Partial pressure \\
\hline $\begin{array}{l}\text { Marchese et al. } \\
(2019)\end{array}$ & Physisorption & $1-80$ & Partial pressure \\
\hline $\begin{array}{l}\text { Kamal K. Pant \& } \\
\text { Upadhyayula }(2019)\end{array}$ & Physisorption & $1-20$ & Partial pressure \\
\hline
\end{tabular}




\subsubsection{1-Olefin Carbon Number Dependent Physisorption}

The physisorbed state is a transition state between the chemisorbed state and fluid phase and is governed by van der Waal's forces (Jager, 1997). From Table 2.2, it has been applied in the work of Todic et al. (2014), Sage et al. (2014), Kamal K. Pant \& Upadhyayula (2019) and Marchese et al. (2019). It has also been used in the 1-olefin re-adsorption product distribution models of van der Laan (1999). The key assumption in these works is that 1-olefins desorb at slower rates with increasing carbon number due to an increase in van der Waal's forces, leading to stronger interactions with the catalyst surface. These forces arise because of 1 -olefin $\pi$-bond interactions with the catalyst surface (Todic et al., 2014; Sage et al., 2014). As such, there is an increase in the activation energy required for desorption of the 1-olefin with carbon number. This leads to an increase in pore residence time, favouring secondary reaction through reinsertion (Todic et al., 2014).

It is known that the heat of adsorption depends linearly on carbon number (van der Laan, 1999). From the Evans-Polanyi principles? this means that the activation energy of the 1-olefin desorption step can be given by Equation 2.10 (Todic et al., 2014; Sage et al., 2014, Kamal K. Pant \& Upadhyayula, 2019). With this assumption, the 1-olefin desorption rate depends exponentially on carbon number in Equation 2.11.

$$
\begin{gathered}
E_{\text {desorption }}=A+B n \\
k_{\text {desorption }}(n, T)=k_{\text {desorption, } 0}(T) \exp \left(\frac{-B}{R T} n\right)
\end{gathered}
$$

The work of Laxmi Narasimhan et al. (2006) on n-paraffin hydrocracking indicates that this desorption behaviour is only observed in the vapour phase. Figure 2.6 shows experimental values of the physisorption equilibrium constant of $n$-paraffins relative to that of $n$-heptane for the vapour and liquid phases. As expected, Figure 2.6 shows that in the vapour phase the relative phisorption equilibrium constant increases exponentially with carbon number. In contrast, for the liquid phase the relative physisorption equilibrium constant is $\approx 1$ and independent of carbon number (Laxmi Narasimhan et al., 2006). This means that there is no preferential physisorption from the liquid phase. In FT synthesis, there is a shift from the vapour to liquid phase with increasing carbon number and the formation of a liquid phase fills the catalyst pores with liquid (Visconti, 2014). This indicates that preferential physisorption is unlikely to cause the observed carbon number dependence since it has no effect on species reactivity. The shift from the vapour to liquid phase with carbon number indicates that solubility is more likely the cause of the observed carbon number dependence.

\footnotetext{
${ }^{7}$ The Evans-Polanyi principle is a method of relating the heat of reaction to the activation energy required for reaction (Fogler 1999).
} 


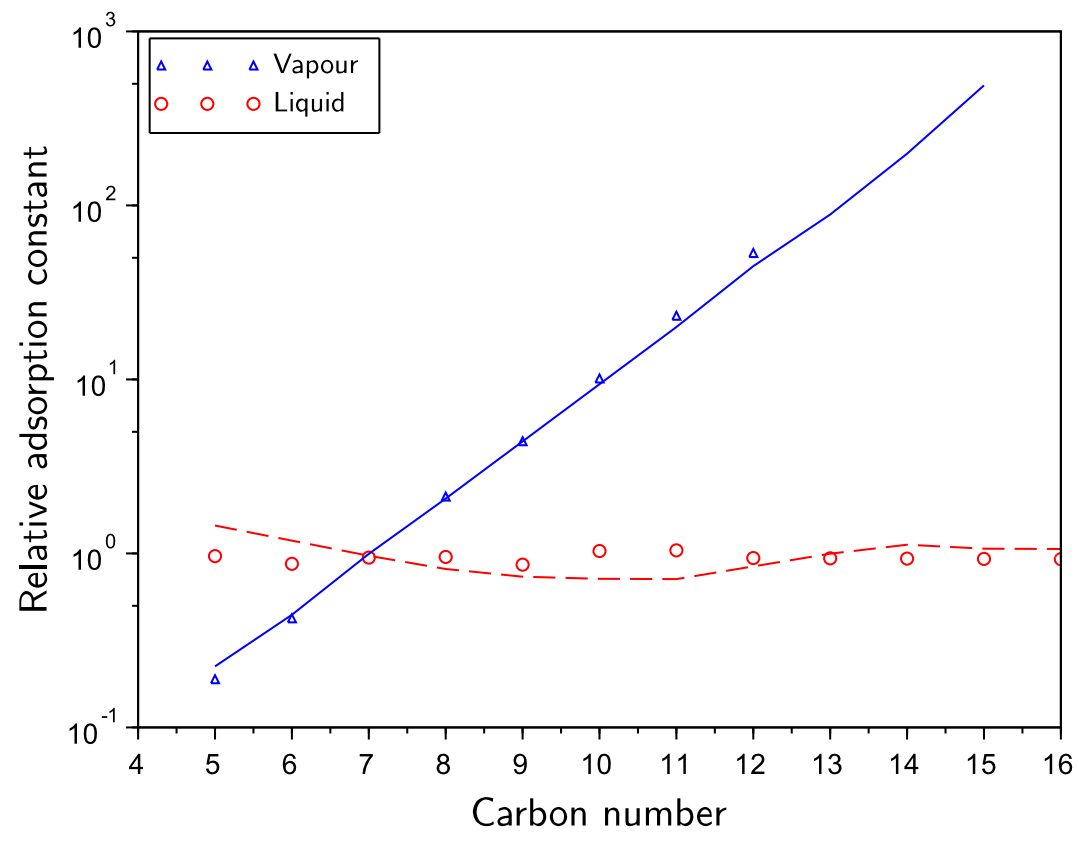

Figure 2.6: Physisorption of the n-paraffins as a function of carbon number at $233^{\circ} \mathrm{C}$ and 80 bar(a), using n-heptane as a reference. Data from Laxmi Narasimhan et al. (2006).

\subsubsection{1-Olefin Carbon Number Dependent Solubility/Vapour-Liquid Equilibrium}

From Table 2.2, the effect of solubility on the rate of desorption has been applied in the models of Visconti et al. (2007), Visconti et al. (2011) and Kwack et al. (2011). It has also been used in the 1-olefin re-adsorption product distribution models of van der Laan (1999) and Schulz \& Claeys (1999). The focus of this approach is the exponential dependence of 1-olefin solubility in the liquid-filled catalyst pores. The model of Kwack et al. (2011) incorporated this effect using Raoult's law to modify the adsorption equilibrium constant. Alternatively, the models of Visconti et al. (2007) and Visconti et al. (2011) developed an explicit correlation, Equation 2.12, based on prior vapour-liquid equilibrium calculations using the Soave-Redlich-Kwong equation of state (EOS). This was used to estimate the liquid phase mole fraction of the 1-Olefin $x_{O_{n}}$ based on its partial pressure $P_{O_{n}}$ in the vapour phase.

$$
x_{O_{n}}=P_{O_{n}} \exp \left(\beta n+\frac{\gamma}{T} n\right)
$$

This expression is analogous to the expression for the desorption rate in Equation 2.11. As the two types of equilibria have similar effects, it is possible for one to be mistaken for the other (Le Grange, 2009). The kinetic driving force used in the model of Visconti et al. (2011) is the liquid phase mole fraction. However, separate correlations are used to estimate the liquid phase mole fractions of 
$\mathrm{CO}, \mathrm{H}_{2}$ and 1-olefins, with no indication of their treatment of the n-paraffins. This indicates that their approach to modelling VLE is questionable i.e. are the liquid phase mole fractions normalised to sum to one? From this, it is can be concluded that while the carbon number dependence of 1olefin reinsertion most likely arises through solubility/VLE, a more consistent treatment is required so that its effect on the observed kinetic behaviour can be directly integrated.

\subsubsection{Model Limitations}

A major limitation of the models in Table 2.2 is their truncation of the carbon number distribution, including only products of low carbon number in their simulation e.g. the models of Todic et al. (2014), (Sage et al., 2014) and Kamal K. Pant \& Upadhyayula (2019). These authors indicated that only carbon numbers 15-20 were present in significant amount which allowed them to neglect heavier products. Only the models of Visconti et al. (2007), Visconti et al. (2011) and Marchese et al. (2019) account for heavy hydrocarbon formation. All of the models in Table 2.2 are complex and when only low carbon number products are accounted for, the model will not be able to correctly predict the product distribution. Truncation of the product distribution by including only products of low carbon number will lead to an over-estimation of the long chain species. This is since a barrier exists in the chain growth reactions (Khazali, 2018).

In the models of Todic et al. (2014), Sage et al. (2014) and Kamal K. Pant \& Upadhyayula (2019), this is not immediately obvious since a set of assumptions e.g. low surface coverage of long chains have been introduced to derive rate expressions in the form of Equation 2.9. Rate expressions of this form do not preserve the polymerisation character of the reaction and as such, it is uncertain whether their extrapolation to higher carbon numbers remains valid. These models cannot be used to extract kinetic parameters as they over-estimate or altogether neglect long chain species. They are only useful in the case of Marchese et al. (2019) where a detailed product analysis was carried out and as such the model formulation accounts for high carbon numbers.

The model formulation of Visconti et al. (2007) and Visconti et al. (2011) retains the polymerisation character of FT synthesis. While products of carbon number up to $\mathrm{C}_{49}$ were included in their simulations, it appears this limit was chosen based on their product analysis. A certain degree of feedback truncation still occurs. This indicates that a more thorough methodology is required regarding when to truncate the product distribution.

Khazali (2018) used Equation 2.5 to determine an appropriate cut-off point. From Figure 2.7, the sum of the product weight fractions is not always guaranteed to equal 1 . This would only be the case if the entire product carbon number range is accounted for since chain growth is indefinite. However, Figure 2.7 shows that careful choice of the maximum carbon number will result in the 
summation being treated as equal to one due to the limit of machine precision. From Figure 2.7. this choice should be based on the expected $\alpha$. For low $\alpha$, there is a greater distribution among the lighter carbon numbers. As such, a low maximum carbon number would be required to ensure a significant fraction of the product distribution is accounted for. However, in LTFT synthesis, high $\alpha$ is expected, with most of the product distributed amongst heavier hydrocarbons. A larger maximum carbon number is therefore required. Figure 2.7 indicates that the summation may always be pushed towards one by increasing the maximum carbon number, while still preserving the character of the product distribution.

It should be noted that if carbon number $n$ is the specified truncation point, it is necessary to extend this cut-off to carbon number $n+1$. This prevents carbon number $n$ from acting as a barrier in the chain growth reactions thus mitigating the error in its estimation (Khazali, 2018).

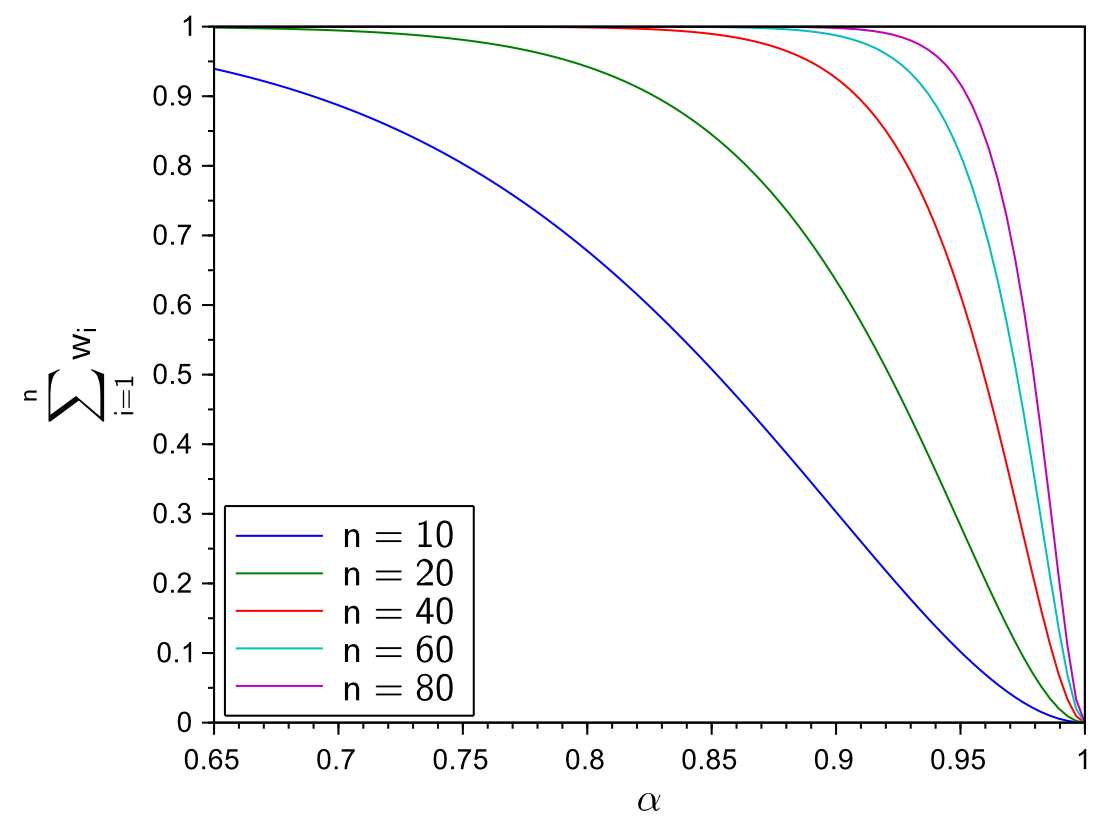

Figure 2.7: Sum of the product weight fractions as a function of $\alpha$ predicted by Equation 2.5 adapted from Khazali (2018)

It is important to note that all these models are assessed based on their ability to account for deviations from the ASF distribution. Many assumptions are made to account for these deviations despite the reasons behind them remaining speculative (Puskas \& Hurlbut, 2003). While this indicates doubt about which model can be used for reliable reactor design, it does indicate that FT reaction behaviour cannot be described by kinetics alone (Glasser et al., 2012), and that it is important to preserve the polymerisation character of the reaction. The models in Table 2.2 
also study the effect of a limited range of conditions on the product distribution to account for deviations from the ASF distribution. This emphasises the need to study the effect of temperature, pressure and $\mathrm{H}_{2} / \mathrm{CO}$ feed ratio on the product distribution as a function of conversion over the entire CO conversion range.

\subsubsection{Modelling Vapour-Liquid Equilibrium (VLE)}

Many FT reactor and kinetic models ignore the presence of VLE in FT synthesis. Caldwell \& van Vuuren (1986) developed criteria for predicting liquid phase formation and the fraction of the product that will condense. It was established that a liquid phase will form once the dewpoint of the reacting mixture is reached. This occurs when the ASF distribution and vapour pressure curves intersect (Caldwell \& van Vuuren, 1986). The dewpoint is reached if $\alpha \geq 0.653$. Since in commercial LTFT operation $\alpha$ is significantly greater (Espinoza et al., 1999; Claeys \& van Steen, 2004), pure vapour phase FT cannot be carried out. This highlights the importance of accounting for the liquid phase.

It is generally assumed that the amount of liquid (wax) formed is small, is in equilibrium with the vapour phase and that the reaction occurs close to the interface between the vapour and liquid phases (Fogler, 1999: Wang et al., 2003a). Therefore, it is essential to model the effect that VLE has on the rate of reaction. Generally, VLE is modelled by assuming Raoult's law applies or an EOS, such as the Peng-Robinson (PR)-EOS, is used.

The work of Masuku et al. (2011) and Masuku et al. (2012a) used Raoult's law to model the effect VLE has on the FT product distribution in a slurry reactor. Flash calculations were carried out using the mass-equilibrium-summation equation set. Their work showed that VLE is attained inside the reactor. Raoult's law was used because both the vapour and liquid phases show nearly ideal behaviour. However, significant deviations from Raoult's law were reported for hydrocarbons of carbon number greater than $C_{18}$. This was attributed to diffusion limitations (Masuku et al., 2011). The effect of diffusion is, however, not the dominant factor influencing the observed product distribution. It is more likely that Raoult's law is no longer valid upon extrapolation to predict the behaviour of heavy hydrocarbons and cannot account for the behaviour of mixtures consisting of molecules of varying size. This indicates that using an EOS together with an appropriate mixing rule may be required (Masuku et al., 2012a). The work of Ahón et al. (2005), Mthombeni (2009), Visconti (2014) and the n-paraffin hydrocracking work of Le Grange (2009) all use the PR-EOS to model VLE.

Visconti (2014) performed similar calculations to Masuku et al. (2011) in a fixed-bed reactor (FBR). Their work showed that $\mathrm{CO}, \mathrm{H}_{2}$ and water were entirely in the vapour phase together 
with the short chain hydrocarbons at $230^{\circ} \mathrm{C}$ and 20 bar(a). Species of carbon number less than $\mathrm{C}_{20}$ are almost entirely in the vapour phase, while $\mathrm{C}_{31+}$ species are almost entirely in liquid phase. Species of carbon number $\mathrm{C}_{20}-\mathrm{C}_{30}$ distribute between the vapour and liquid phases. The model also predicted that $99.97 \mathrm{~mol} . \%$ of the species are in the vapour phase. This is, however, at low CO conversion $(\approx 15 \%)$ but it indicates why most FBR models consider only the vapour phase (Satterfield et al., 1985). A major shortcoming of these flash calculations is that all $\mathrm{C}_{31+}$ species are lumped together due to their thermo-chemical properties not being available in open literature (Visconti, 2014). This is not justified when various estimation methods exist. This does emphasise the need to develop an extensive physical property database, suited to extrapolation to high carbon numbers. It may aid in providing refined estimates regarding the true liquid content of the reactor.

Unlike the models of Masuku et al. (2011), Masuku et al. (2012a) and Visconti (2014), the models of Ahón et al. (2005), Mthombeni (2009) and Le Grange (2009) carry out simultaneous VLE and kinetic calculations within their reactor models. The work of Ahón et al. (2005) and Mthombeni (2009) used the mass-equilibrium-summation equation set which is modified to account for reaction in a slurry reactor. These authors, however, both indicate long solution times for this method due to the large number of equations, one for each species, that needs to be solved. The model of Le Grange (2009), however, used the Rachford-Rice algorithm, Equation 2.13, for all flash calculations in an FBR. This reduces many non-linear equations down to a single equation, speeding up solution time by several orders of magnitude (Le Grange, 2009). This appears to be the most effective way to account for the effect of VLE on the reaction kinetics.

$$
\sum_{i=1}^{n_{\text {species }}} \frac{\left(K_{i}-1\right) z_{i}}{1+\left(K_{i}-1\right) \psi}=0
$$

VLE and kinetics should, however, not be treated separately (Mthombeni, 2009). From Table 2.2. the most commonly used kinetic driving force is species partial pressure. A different approach is required to ensure that kinetic and thermodynamic models are coupled and dependent on each other. The rate expressions should be written as a function of species fugacity/activity (Ahón et al., 2005; Laxmi Narasimhan et al., 2006; Mthombeni, 2009). Activity is defined as the ratio of a species fugacity in a mixture relative to the fugacity of it as a pure species at standard pressure in Equation 2.14. This directly incorporates the effect VLE has on the rate of reaction Mthombeni, 2009).

$$
a_{i}=\frac{f_{i}}{f_{i}^{\circ}}
$$




\subsubsection{Chemical Equilibrium Considerations}

Overall chemical equilibrium calculations indicate methane is the most stable hydrocarbon product. This means that the thermodynamic driving force to form methane is so high that the formation of long chain hydrocarbons must be kinetically controlled (Masuku et al., 2012b). With this in mind, it has resulted in FT kinetic work focusing on using mechanistic features of the reaction to explain the observed behaviour. This continued search for a kinetic explanation ignores that while FT reactions may not be controlled by overall chemical equilibrium, there are aspects of it that may be partially controlled by equilibrium (Norval \& Phillips, 1990).

Overall chemical equilibrium calculations consider all species present and all possible chemical reactions that may occur in a system (Norval \& Phillips, 1990). The system is stoichiometrically unrestricted and depends only on the Gibbs free energy of the chemical species present. Like overall chemical equilibrium, partial chemical equilibrium depends only on the latter. The difference, however, is that only a subset of the chemical species attains equilibrium (Norval et al., 1992). Both overall and partial chemical equilibrium may be stoichiometrically restricted in that not all reactions may be involved. When applied to the FT reactions for the main products, this means that $n$-paraffins and 1-olefins are at equilibrium within their respective homologous series, but not with each other (Norval \& Phillips, 1990).

By assuming partial chemical equilibrium exists in the n-paraffin and 1-olefin homologous series, ideal gas behaviour and applying the Gibbs free energy minimisation technique 8 of Smith \& Missen (1988), Equations 2.15 and 2.16 were derived for $n$-paraffins and 1-olefins respectively (Norval \& Phillips, 1990; Norval, 2008).

$$
\begin{gathered}
\ln \left(y_{n, P}\right)=n\left(\lambda_{C, P}+2 \lambda_{H, P}-\frac{B_{P}}{R T}\right)-\frac{A_{P}}{R T}-\ln (P)+2 \lambda_{H, P} \\
\ln \left(y_{n, O}\right)=n\left(\lambda_{C, O}+2 \lambda_{H, O}-\frac{B_{O}}{R T}\right)-\frac{A_{O}}{R T}-\ln (P)
\end{gathered}
$$

Equations 2.15 and 2.16 are based on the linear relationship between the ideal gas Gibbs free energy of formation and carbon number at constant temperature in Equation 2.17.

$$
\Delta G_{f, j}^{\circ}(n, T)=A_{j}(T)+B_{j}(T) n
$$

The Lagrange multiplier, $\lambda_{i, j}$, refers to the atomic species $i$ in the homologous series $j$. This indicates that homologous series are in equilibrium amongst themselves but not with one another

\footnotetext{
${ }^{8} \mathrm{~A}$ technique applied to solve chemical equilibrium problems by formulating it as a constrained optimisation problem. The objective function is the total Gibbs free energy of the system.
} 
i.e. at partial equilibrium. Overall chemical equilibrium therefore does not occur. The overall distribution considering the main products can be obtained by recognising that $y_{n}=y_{n, P}+y_{n, O}$, $B \equiv B_{P} \cong B_{0}{ }^{9}$ and assuming that $\left(\lambda_{C}+2 \lambda_{H}\right) \equiv\left(\lambda_{C, P}+2 \lambda_{H, P}\right) \cong\left(\lambda_{C, O}+2 \lambda_{H, O}\right)$ to give Equation 2.18 (Norval \& Phillips, 1990).

$$
\ln \left(y_{n}\right)=n\left(\lambda_{C}+2 \lambda_{H}-\frac{B}{R T}\right)-\ln (P)+\ln \left(\exp \left(\frac{-A_{O}}{R T}\right)+\exp \left(\frac{-A_{P}}{R T}+2 \lambda_{H, P}\right)\right)
$$

The form of Equation 2.18 is analogous to the linearised form of the ASF distribution. This indicates that the ASF distribution can be derived from thermodynamics as shown in Figure 2.8 . The implications of the thermodynamic basis for the ASF distribution indicates that the FT reaction system consists of a number of subsystems working towards achieving partial equilibrium.

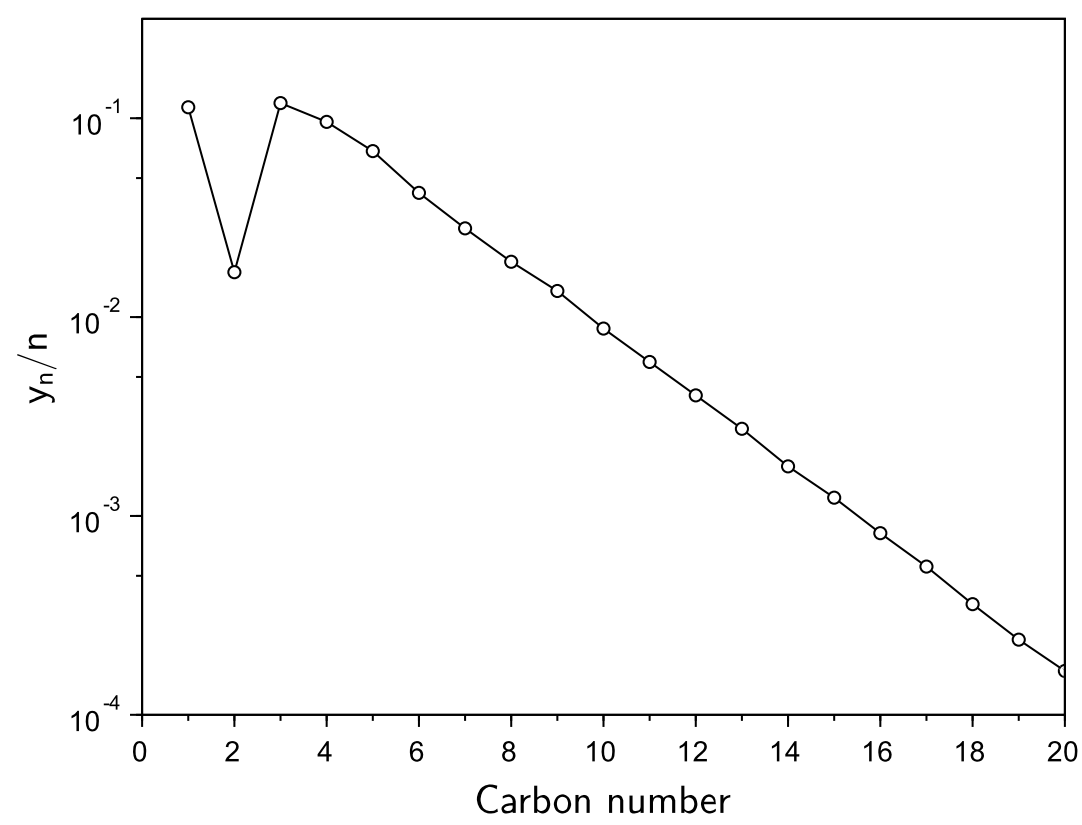

Figure 2.8: Molar ASF distribution derived from thermodynamics at $327^{\circ} \mathrm{C}$ and 10 bar(a) adapted from Norval \& Phillips (1990)

Figure 2.8 demonstrates that partial equilibrium would occur if thermodynamics controlled the distribution of hydrocarbons but kinetics controlled their yield (Norval \& Phillips, 1990). Identifying partial equilibrium is useful because it results in a reduction in the number of rate expressions that would be required during reactor and kinetic modelling (Norval et al., 1992). This means that if the $\mathrm{FT}$ reaction is considered as equilibrium-limited process, then the kinetic model would consist

\footnotetext{
${ }^{9}$ This is the case for $\mathrm{n}$-paraffin and 1-olefins because each compound in either homologous series differs only by the addition of another $\mathrm{CH}_{2}$ group (Norval \& Phillips, 1990).
} 
of simple rate expressions describing hydrocarbon formation with a few adjustable parameters, without compromising on prediction quality. This emphasises that equilibrium aspects also need to be considered before attributing observations to kinetic effects and building a complex mechanisticbased model (Norval, 2008). The interaction between VLE, partial chemical equilibrium and kinetics may help to model the observed behaviour in the FT reaction system (Glasser et al., 2012).

\subsubsection{Influence of Process Conditions on Selectivity}

Since FT synthesis is a polymerisation reaction, selectivity can only be 'controlled' in a limited manner. This is most often done through $\alpha$ by knowing its dependence on process conditions (Claeys \& van Steen, 2004). While the FT reactions will invariably give rise to a mixture of hydrocarbons of different carbon number, the appropriate choice of catalyst and process conditions enables $\alpha$ to be shifted such that the desired product range can be obtained (Sie et al., 1991). The desired $\alpha$ can be obtained by varying reaction temperature, pressure, $\mathrm{H}_{2} / \mathrm{CO}$ feed ratio and operating at an appropriate CO conversion level.

Low temperatures result in higher $\alpha$ values from the definition in Equation 2.6 and as such a product of higher average molecular weight. This can be mechanistically interpreted as an inhibition of the termination step relative to the chain growth step (Claeys \& van Steen, 2004). Increasing pressure favours selectivity towards heavier and more oxygenated products (Espinoza et al., 1999). The $\mathrm{H}_{2} / \mathrm{CO}$ feed ratio is often not seen as a process parameter since it cannot be varied over a wide range (Claeys \& van Steen, 2004). Generally, however, lowering the $\mathrm{H}_{2} / \mathrm{CO}$ feed ratio favours a heavier FT product. It follows that higher $\mathrm{H}_{2} / \mathrm{CO}$ feed ratios results in a lighter product and a lower olefin content due to high $\mathrm{H}_{2}$ contents (Iglesia et al., 1993).

An important consideration when operating the reactor is the choice of residence time. This meaning knowing the relationship between product selectivity and $\mathrm{CO}$ conversion. An important consideration in a syngas conversion plant is the wax selectivity which is often measured as $\mathrm{C}_{5+}$ (van der Laan, 1999; Claeys \& van Steen, 2004). Since it is not possible to reduce the rate of formation of methane, it is necessary to maximise the rate of formation of wax relative to that of methane. This ensures favourable $C_{5+}$ selectivity is obtained (Claeys \& van Steen, 2004; van Steen et al., 2018).

It is generally acknowledged that an increase in CO conversion results in an increase in the average molecular weight of the product (Iglesia et al., 1991). This is because secondary reactions are directly influenced by residence time i.e. the extent of reinsertion is greater with CO conversion. This corresponds to a decrease in the OPR with an increase in CO conversion. These conclusions are limited in that it lacks the necessary detail about what occurs over the full CO conversion 
range.

The work of Tucker \& van Steen (2019) investigated the effect of CO conversion, in the range $15-97 \%$, on the selectivity to methane, $\mathrm{C}_{5+}$ and $\mathrm{CO}_{2}$ in a slurry reactor over a Co- $\mathrm{Pt} / \mathrm{Al}_{2} \mathrm{O}_{3}$ catalyst. The use of a slurry reactor results in isothermal conditions which allows the effect of $\mathrm{CO}$ conversion solely to be studied. With increases in CO conversion in the range 20-75\%, a decrease in methane selectivity was observed. In contrast, $C_{5+}$ selectivity was observed to increase slightly, before levelling off until a $\mathrm{CO}$ conversion of $\approx 80 \%$. Further increases in $\mathrm{CO}$ conversion from $75-97 \%$ led to a strong increase in methane selectivity corresponding to a sharp decline in $\mathrm{C}_{5+}$ selectivity. A steady, followed by a more rapid rise in $\mathrm{CO}_{2}$ selectivity was observed over the $\mathrm{CO}$ conversion range studied.

These observations were attributed to catalyst deactivation due to re-oxidation through exposure to high water partial pressures at high CO conversion, with phase changes in the catalyst leading to WGS activity (Bukur et al., 2012; Tucker \& van Steen, 2019). Catalyst deactivation due to re-oxidation by water is consistent with Sasol studies on Co-based catalysts at high per pass CO conversion (Espinoza et al., 1999). This indicates that the higher $C_{5+}$ productivity advantage of Co-based catalysts at higher CO conversions can only be sustained up to the point where water inhibition becomes significant. With an increase in WGS activity, there was an increase in the $\mathrm{H}_{2} / \mathrm{CO}$ ratio. Consequently, lighter products became more favourable leading to the observed decrease in $\mathrm{C}_{5+}$ selectivity. Some cracking of hydrocarbons has also been observed at high $\mathrm{CO}$ conversion and could also have occurred (Iglesia, 1993). The increase in methane selectivity is expected due to the large thermodynamic driving force to form methane at high CO conversion (Masuku et al., 2012b).

This work therefore demonstrates that $\mathrm{C}_{5+}$ selectivity increases up to a point with increasing $\mathrm{CO}$ conversion before the thermodynamic driving force to form methane, the most stable hydrocarbon product, dominates at high $\mathrm{CO}$ conversion. This implies that an optimal conversion range exists which maximises selectivity to $\mathrm{C}_{5+}$ relative to that of methane and highlights the importance of conducting FT synthesis under kinetically controlled conditions. The complex interplay between process conditions reinforces the need for a kinetic and reactor model that can accurately model not just the observed kinetic occurrence but also the effect of process conditions on $\mathrm{C}_{5+}$ selectivity, since this is crucial to overall FT process economics (van der Laan, 1999).

\subsection{Summary and Insights Gained}

FT synthesis is a polymerisation reaction and under LTFT conditions, the main products are linear n-paraffins and 1-olefins. All the metals used as FT catalysts are active for hydrogenation 
reactions. Fe- and Co-based catalysts are used commercially. The higher wax productivity of Co-based catalysts at higher per pass conversions and the higher activity at lower temperature and pressure makes it more suitable for industrial LTFT processes. Additionally, high n-paraffin yields are obtained with a low WGS activity resulting in more effective carbon utilisation to form FT products with improved thermal efficiency. While the mechanistic details of the FT reactions remain controversial, there is agreement that it is a chain polymerisation reaction. Chain growth occurs through the addition of a single $C_{1}$ intermediate at the end of the hydrocarbon chain. The mechanistic situation can be simplified by considering only the main products. For n-paraffin and 1-olefin formation, the alkyl mechanism is most plausible.

The polymerisation character of FT synthesis means that the nature of the product spectrum can be estimated using the ASF distribution given by Equation 2.5. This allows the product distribution of FT synthesis to be characterised by a single parameter i.e. $\alpha$. High $\alpha$ values are desired in LTFT synthesis, which indicates that the product is highly paraffinic in nature. In practise, deviations from the ideal ASF distribution are observed, especially in LTFT synthesis. The main deviations are a high methane yield, low ethene yield and a change in $\alpha$ for $n$-paraffins, corresponding to an exponential decrease in the OPR with carbon number.

The high methane yield is a result of the large thermodynamic driving force to form methane. Methane formation can be controlled kinetically using temperature and pressure conditions. There is qualitative agreement that the occurrence of 1-olefin secondary reactions, specifically reinsertion, gives rise to the observed selectivity character of FT synthesis. However, reinsertion alone cannot lead to the observed deviations. It is rather a combination of reinsertion and carbon number dependent processes that influence its extent which lead to the observed deviations. Carbon number dependent diffusion, physisorption and solubility/VLE affects the extent of reinsertion. It is most likely that the dominant process involved is solubility/VLE.

The most effective way to model the effect that VLE has on the kinetic behaviour of FT synthesis is through an appropriate EOS. The PR-EOS is the most widely used formulation. There is a need, however, for an extensive physical property database suitable for extrapolation to high carbon numbers. Kinetic and thermodynamic models need to be integrated so that they are dependent on one another. This can be done by using the species activity as the kinetic driving force. Of the two approaches used to model FT kinetics, a mechanistic-based approach for n-paraffin and 1-olefin formation, based on the alkyl mechanism, is most viable to allow for simultaneous description of reactant consumption and product formation. As such, a prediction of product selectivity is made. It is, however, important that the model preserves the polymerisation character of FT synthesis.

A major limitation of most models in literature is that a sizeable fraction of the product distribution 
is not accounted for. It is important to include a detailed simulation of the product distribution to prevent feedback truncation leading to over-estimation of the high carbon number product quantities. This reinforces the need to preserve the polymerisation character of the reaction in the kinetic model formulation. This will allow for reliable estimates of the kinetic parameters to be extracted from experimental data. Most models also only study the effect of conditions in a limited range, with emphasis on accounting for deviations from the ASF distribution. There is a need to study the effect of temperature, pressure and $\mathrm{H}_{2} / \mathrm{CO}$ feed ratio on the product distribution as a function of $\mathrm{CO}$ conversion over the full $\mathrm{CO}$ conversion range.

It is also important to consider the equilibrium aspects which may be involved in the FT reaction system before assuming that the observed characteristics only have a kinetic explanation. The thermodynamic basis of the ASF distribution indicates that the $n$-paraffin and 1-olefin products are separate subsystems working to achieve partial chemical equilibrium. The implication of this is that the yield of these hydrocarbons could be controlled kinetically with thermodynamics controlling their distribution. The usefulness of identifying partial equilibrium in the FT reaction system means that the kinetic model could be simplified and consist of fewer rate expressions. The model would have a few adjustable parameters without compromising on prediction quality while maintaining thermodynamic consistency. The interaction between VLE, partial chemical equilibrium and kinetics may help to model the characteristics of the FT reaction system. 


\section{Chapter 3}

\section{Thermo-physical Considerations}

\subsection{Physical Property Estimation}

\subsubsection{Theory}

When modelling FT synthesis, the properties of n-paraffins and 1-olefins need to be known or accurately estimated (Marano \& Holder, 1997d). The physical properties of low carbon number hydrocarbons are known because experimental data can easily be obtained and they are common in commercial processes. It is challenging to obtain accurate experimental data for their higher carbon number relatives because they can degrade chemically at their very high critical temperatures (Poling et al., 2001). This is the case for $n$-paraffins while 1-olefins begin to polymerise at a certain point (Marano \& Holder, 1997b). The physical property database of n-paraffins and 1-olefins for high carbon numbers is therefore fragmentary, with some data not available at all (Derevich et al., 2008). The missing physical properties of n-paraffins and 1-olefins can, however, be estimated using either an appropriate estimation method or by correlating existing data (Marano \& Holder, 1997d; Poling et al., 2001).

The most common physical property estimation method involves using group contributions. This is since all macroscopic properties are related to molecular structure and the bonds between atoms. The latter determines the strength and type of intermolecular forces present (Poling et al., 2001). The principle behind any group contribution method (GCM) is that while the number of chemical species that exist is large, the subset of their elemental or molecular group building blocks is significantly smaller (Elliot \& Lira, 2012). These properties are calculated using an algebraic expression that sums the group contributions that make up a molecule (Poling et al., 2001). 
An advanced GCM, based on the UNIFAC 1 functional groups, is the method of Constantinou and Gani (CG) (Poling et al., 2001). The general form of the function $f$ used to estimate the physical property $F$ in the CG method is given by Equation 3.1 .

$$
F=f\left(\sum_{k=1}^{n_{\text {groups }}} N_{k}\left(F_{1, k}\right)+W \sum_{j=1}^{n_{\text {groups }}} M_{j}\left(F_{2, j}\right)\right)
$$

In Equation 3.1, $f$ can be a linear or non-linear function, $N_{k}$ is the number of first order groups of type $k$ in the molecule, $F_{1, k}$ is the contribution of the first order group $1, k$ specific to the property $F, M_{j}$ is the number of second order groups of type $j$ in the molecule and $F_{2, j}$ is the contribution of the second order group 2, $j$ (Poling et al., 2001). The value of $W$ is set to zero for first order calculations and one for second order calculations. For linear n-paraffins and 1-olefins, the second order contribution can be neglected.

The CG method is reliable when used to estimate critical and ideal gas properties. This method, however, produces poor estimates of the physical properties for the smallest and largest molecules (Poling et al., 2001). The former occurs because group contribution is not so accurate for small molecules, even if it is possible to form them from the available groups. The latter may be a consequence of a failure to predict the limiting behaviour of physical properties for very large molecules (Marano \& Holder, 1997d). The predictive ability of the CG method across the entire carbon number range can be improved by making small modifications. Failings of the method may occur as a result of using generic values of the functional group contributions. Physical property predictions can be improved by correlating available experimental data directly, where the adjustable parameters are the group contributions. This ensures that the value of the functional group contribution is specific to the n-paraffin and 1-olefin structures. The functional form $f$ of the property $F$ is retained and the minimum number of input parameters will be required. This will improve predictions by the CG method, especially when extrapolating to high carbon numbers.

\subsubsection{Methodology and Assumptions}

To determine values of the group contributions for use in the CG method, specific to the n-paraffin and 1-olefin structures, a reference physical property database is required. This is obtained from the pure component database of the open source version of ChemSep LITE v8.14 (www.chemsep.com). These data are assumed to be of reasonable accuracy to estimate the group contributions through regression. The physical property data of the $C_{1}-C_{29}$ n-paraffins and $C_{2}-C_{9}$ and $C_{11}$ 1-olefins are available in the database. The required properties are the critical pressure, critical temperature,

\footnotetext{
${ }^{1}$ The universal quasi-chemical (UNIQUAC) functional group activity coefficient (UNIFAC) model is a predictive model that uses the functional groups present in a non-ideal liquid mixture to calculate activity coefficients.
} 
normal boiling point, critical volume and acentric factor. In addition, the ideal gas heat of formation, Gibbs free energy of formation and heat capacity are also required. The ideal gas heat capacity is a function of temperature. The equation for heat capacity in the pure component database is assumed to correlate the data well and as such, serves in place of temperature-dependent experimental data. The ideal gas heat capacity was regressed over the temperature range 298.15-623.15 K. The lower bound of the temperature range is chosen because it is a common reference point for most thermodynamic calculations. The upper bound of the temperature range is chosen as conservative limit to ensure that the correlation remains valid over a wide range of LTFT reactor operation.

For all physical properties, the functional form $f$ given by the CG method for the property $F$ is retained. The only adjustable parameters are the group contributions and, in certain cases (critical pressure, critical temperature and normal boiling point), some of the numerical values included as part of the function $f$ in the CG method to improve the correlation to existing data. This ensures that the desired physical properties exhibit consistency when extrapolated. This means that the difference between the physical properties of n-paraffins and 1-olefins should diminish and approach the same limiting value at high carbon number as the long chain effects dominate relative to the functional group effects (Marano \& Holder, 1997d). Since linear n-paraffins and 1-olefins are the main products of FT synthesis, the second order contribution is neglected. The CG method can only be applied to n-paraffins of carbon number $n \geq 2$ and 1-olefins of carbon number $n \geq 3$ using the functional groups available in Poling et al. (2001). As such, only the physical properties of these species will be used in the regression procedure. The first order contribution for $n$-paraffins and 1 -olefins is given by Equation 3.2 and 3.3 respectively.

$$
\begin{gathered}
2 F_{1, C H_{3}}+(n-2) F_{1, C H_{2}} ; n \geq 2 \\
F_{1, C H_{2}=C H}+(n-3) F_{1, C H_{2}}+F_{1, C H_{3}} ; n \geq 3
\end{gathered}
$$

In Equation 3.2 and 3.3, the contribution by the functional groups $\mathrm{CH}_{3}$ and $\mathrm{CH}_{2}$ are assumed to be identical in both linear n-paraffin and 1-olefin structures. As such, only the $\mathrm{CH}_{2}=\mathrm{CH}$ group needs to be determined when regressing the physical properties of the 1-olefins.

The values of the group contribution which correlate the required physical properties of $n$-paraffins, and 1-olefins are obtained by minimising the objective function in Equation 3.4. Here, $n_{\text {data }}$ refers to the total number of data points, $Y_{\text {exp,i }}$ refers to the experimental physical property value and $Y_{c a l c, i}$ is the value predicted by the correlation. To minimise Equation 3.4, the Isqrsolve function in Scilab, based on the Levenberg-Marquardt algorithm, is used. All errors in regressed parameters are estimated by assuming that it follows a normal distribution. This assumes that the model fits 
the data well i.e. has a small residual.

$$
\text { minimise : } \quad f_{\text {objective }}=\sum_{i=1}^{n_{\text {data }}}\left(Y_{\text {exp }, i}-Y_{c a l c, i}\right)^{2}
$$

\subsubsection{Results and Discussion}

The vapour-liquid critical properties, namely critical pressure, critical temperature, normal boiling point, critical volume and acentric factor are the pure component properties that are used in many correlations to estimate volumetric, thermodynamic and transport properties of gases and liquids (Poling et al., 2001). These properties are also necessary for accurate VLE calculations. Ideal gas properties at $298.15 \mathrm{~K}$ and 1 bar(a) are necessary for establishing the reference state for the calculation of enthalpy at the temperature and pressure of operation and for formulating chemical equilibrium (Marano \& Holder, 1997c).

\subsubsection{Critical Pressure Estimation}

The form of the function used in the CG method to estimate the critical pressure is given by Equation 3.5. In addition to the group contributions, the value of the asymptote in the CG method is also chosen as an adjustable parameter. This is since critical pressure shows limiting behaviour at high carbon number (Marano \& Holder, 1997b). The value of the asymptote will be the same for both n-paraffins and 1-olefins to ensure consistency at the limit of infinite carbon number (Marano \& Holder, 1997d). The group contribution for $n$-paraffins and 1-olefins are given by Equation 3.2 and 3.3 respectively.

$$
P_{c}=\left(\sum_{k=1}^{n_{\text {groups }}} N_{k}\left(p c_{1, k}\right)\right)^{-2}+p c_{\text {asymptote }}
$$

From Figure 3.1, the prediction of the critical pressure using the CG method is excellent, with a very good qualitative fit to the data. The values of the group contributions and the asymptote are estimated with a high degree of confidence in Table 3.1, shown by the small parameter error. The Lattice-Fluid and Flory-Cell models predict zero and non-zero values of the critical pressure asymptote (Marano \& Holder, 1997b). While a non-zero value of the critical pressure asymptote is predicted for n-paraffins and 1-olefins in Table 3.1, this should not be seen as support for either theory but rather as a parameter arising as a result of the empirical best fit to data based on the formulation of Equation 3.5 . 


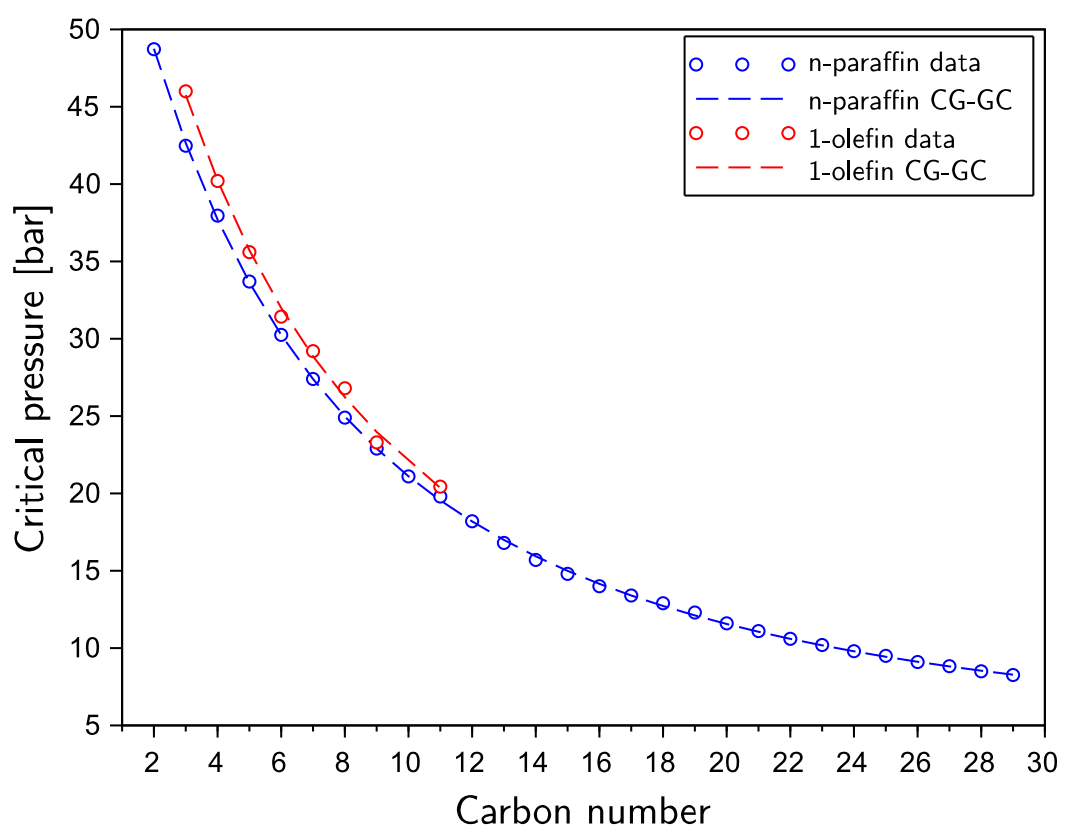

Figure 3.1: Critical pressure prediction by the CG method

Table 3.1: Critical pressure parameters in Equation 3.5

\begin{tabular}{lc}
\hline Parameter & Value \\
\hline$p c_{\mathrm{CH}_{3}}\left[\mathrm{bar}^{0.5}\right]$ & $0.02404 \pm 0.00008$ \\
$p c_{\mathrm{CH}_{2}}\left[\mathrm{bar}^{0.5}\right]$ & $0.01107 \pm 0.00009$ \\
$p c_{\mathrm{CH}_{2}=\mathrm{CH}}\left[\mathrm{bar}{ }^{0.5}\right]$ & $0.02404 \pm 0.00045$ \\
$p C_{\text {asymptote }}[\mathrm{bar}]$ & $3.278 \pm 0.094$ \\
\hline
\end{tabular}

\subsubsection{Critical Temperature Estimation}

The form of the function used in the CG method to estimate the critical temperature is given by Equation 3.6. From Figure 3.2, the prediction of the database critical temperature values is qualitatively good. The values of the group contributions have also been estimated with a high degree of confidence in Table 3.2 .

$$
T_{c}=t c_{\text {coefficient }} \ln \left(\sum_{k=1}^{n_{\text {groups }}} N_{k}\left(t c_{1, k}\right)\right)
$$

Marano \& Holder (1997b) report that the critical temperature of n-paraffins and 1-olefins should approach a limiting value. Unfortunately, a range of limiting critical temperature values exist in literature, varying between $960-1100 \mathrm{~K}$. This large temperature range indicates that the limiting 
value arises as a result of an empirical fit to the experimental dataset used during the regression procedure. There is also no consistent agreement between the Lattice-Fluid and Flory-Cell theories (Marano \& Holder, 1997b). The form of Equation 3.6 suggests that the limiting critical temperature value is infinite. Regardless, the correlation of the data by Equation 3.6 is good and the logarithmic form should result in safe extrapolation to higher carbon numbers.

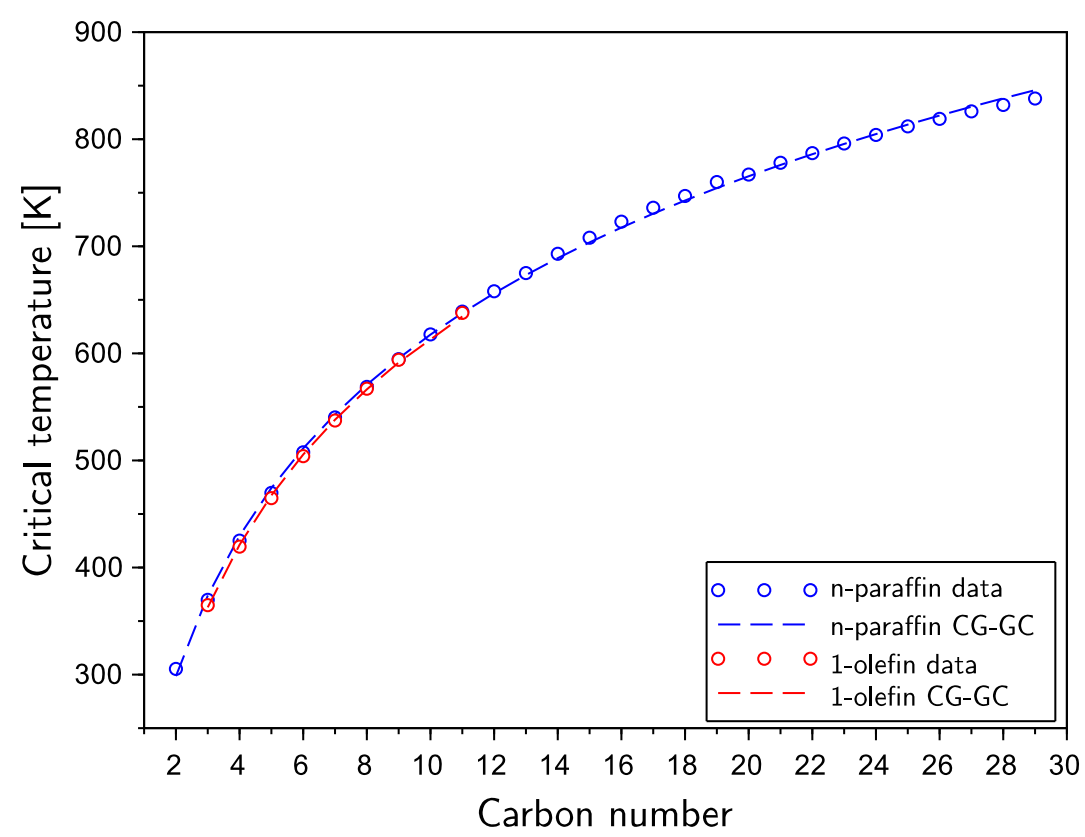

Figure 3.2: Critical temperature prediction by the CG method

Table 3.2: Critical temperature parameters in Equation 3.6

\begin{tabular}{lc}
\hline Parameter & Value \\
\hline$t c_{\mathrm{CH}_{3}}$ & $1.937 \pm 0.035$ \\
$t c_{C H_{2}}$ & $1.574 \pm 0.091$ \\
$t c_{\mathrm{CH}_{2}=\mathrm{CH}}$ & $3.248 \pm 0.031$ \\
$t c_{\text {coefficient }}[\mathrm{K}]$ & $220.5 \pm 3.4$ \\
\hline
\end{tabular}

\subsubsection{Normal Boiling Point Estimation}

Like the critical temperature, the normal boiling point functional form in the CG method is given by Equation 3.6. The same functional form ensures that both the critical temperature and normal boiling point will approach the same limiting value at high carbon numbers and that these two physical properties are consistent relative to one another (Marano \& Holder, 1997d,b). From Figure 3.3. the prediction of the data is excellent which provides confidence regarding the functional form 
given by Equation 3.6 and its predictions when extrapolated for both the critical temperature and normal boiling point. The parameters have also been estimated with a high degree of confidence in Table 3.3 .

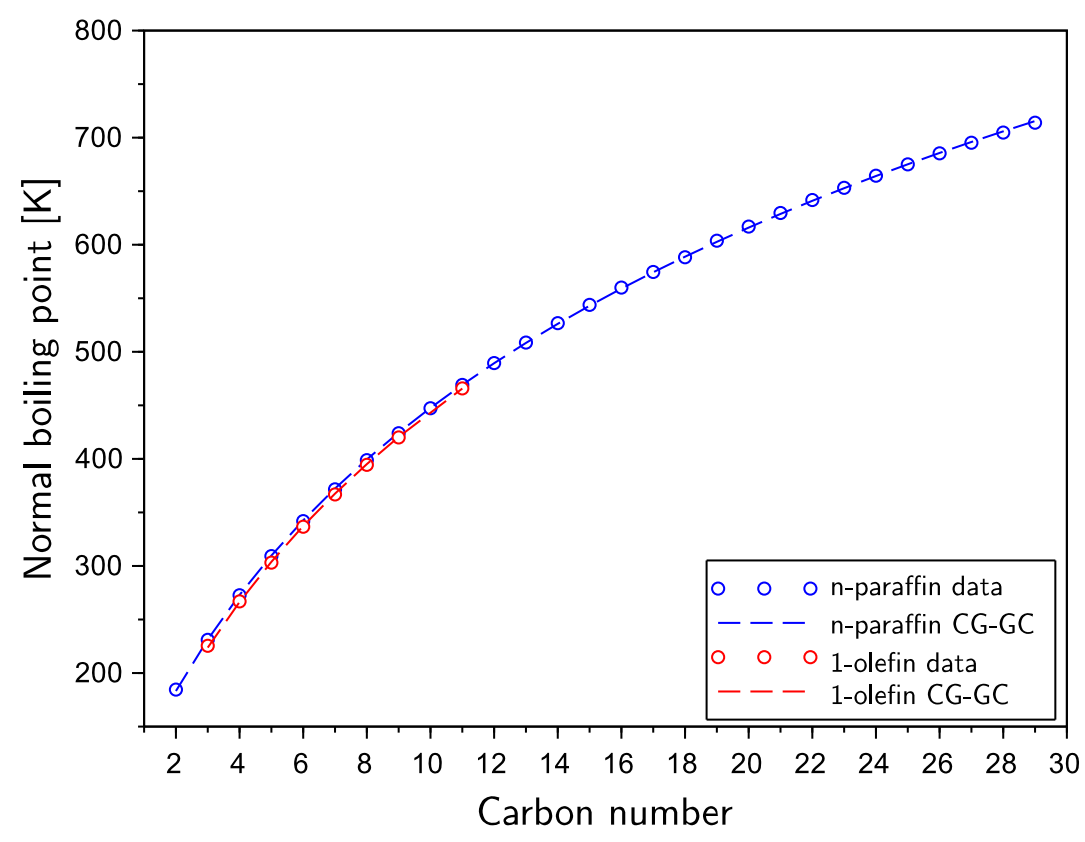

Figure 3.3: Normal boiling point prediction by the CG method

Table 3.3: Normal boiling point parameters in Equation 3.6

\begin{tabular}{lc}
\hline Parameter & Value \\
\hline$t b_{\mathrm{CH}_{3}}$ & $0.8985 \pm 0.0017$ \\
$t b_{\mathrm{CH}_{2}}$ & $0.2987 \pm 0.0031$ \\
$t b_{\mathrm{CH}}=\mathrm{CH}$ & $1.147 \pm 0.003$ \\
$t b_{\text {coefficient }}[\mathrm{K}]$ & $312.6 \pm 1.3$ \\
\hline
\end{tabular}

\subsubsection{Critical Volume Estimation}

The form of the function used in the CG method to estimate the critical volume is given in Equation 3.7. From Figure 3.4, the variation of the critical volume is almost linear with carbon number. This is most evident from carbon numbers $C_{2}-C_{12}$ and from $C_{20}$ onwards for the n-paraffins where considerable errors appear to be present in the critical volume database. This is in contrast to the data in the 1-olefins database, where Equation 3.7 gives better predictions of the data for all carbon numbers. Equation 3.7 should be expected to give more reliable predictions for the $n$-paraffins and should give accurate results when extrapolated for the 1-olefins. The estimated parameters are 
given in Table 3.4 .

$$
V_{c}=-0.00435+\left(\sum_{k=1}^{n_{\text {groups }}} N_{k}\left(v c_{1, k}\right)\right)
$$

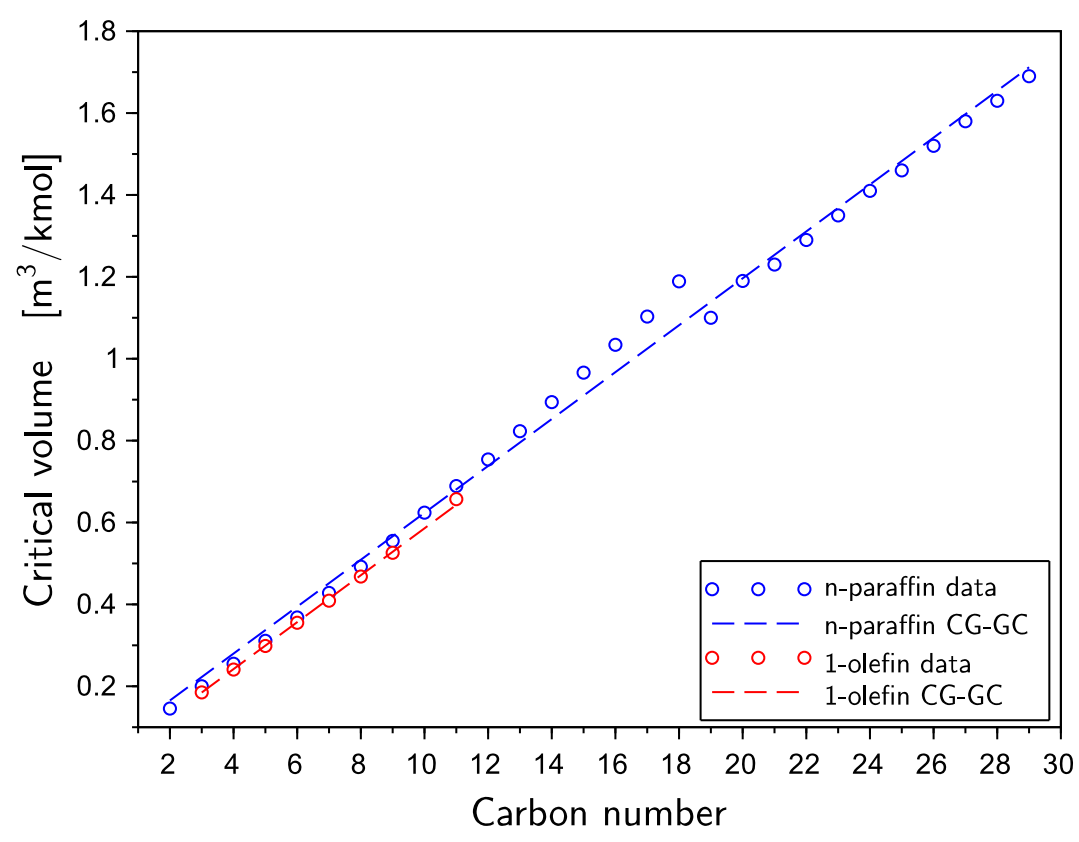

Figure 3.4: Critical volume prediction by the CG method

Table 3.4: Critical volume parameters in Equation 3.7

\begin{tabular}{lc}
\hline Parameter & Value \\
\hline$v c_{\mathrm{CH}_{3}}\left[\mathrm{~m}^{3} / \mathrm{kmol}\right]$ & $0.08482 \pm 0.00698$ \\
$v c_{\mathrm{CH}_{2}}\left[\mathrm{~m}^{3} / \mathrm{kmol}\right]$ & $0.05728 \pm 0.00089$ \\
$v c_{\mathrm{CH}}=\mathrm{CH}\left[\mathrm{m}^{3} / \mathrm{kmol}\right]$ & $0.1043 \pm 0.0021$ \\
\hline
\end{tabular}

\subsubsection{Acentric Factor Estimation}

The acentric factor is a derived property which provides an experimental vapour pressure point that improves the predictive ability of an equation of state and extends its applicability to non-spherical molecules and (Poling et al., 2001; Elliot \& Lira, 2012). Its definition is given in Equation 3.8 where $P^{s a t}$ is the vapour pressure at a reduced temperature of 0.7 . The use of this reference is arbitrary and other reference points have been proposed (see Twu et al. (1994) for example).

$$
\omega \equiv-\log _{10}\left(\frac{P^{s a t}\left(0.7 T_{c}\right)}{P_{c}}\right)-1
$$


The recommended procedure by Poling et al. (2001) for estimating the acentric factor is to use a very accurate correlation for the vapour pressure directly in the definintion given by Equation 3.8. These correlations are not readily available for all n-paraffins and 1-olefins. The next most reliable approach involves estimating the critical pressure, critical temperature and normal boiling point. The vapour pressure is then atmospheric pressure and the acentric factor can be estimated using Equation 3.9, where $T_{b r}$ is the reduced temperature at the normal boiling point (Twu et al., 1994).

$$
\omega=-\frac{\ln \left(\frac{P_{c}}{1.0325}\right)+f_{0}\left(T_{b r}\right)}{f_{1}\left(T_{b r}\right)}
$$

Both of these approaches require estimates of either the vapour pressure or critical properties to be made and as such substantial errors may occur in the estimation of the acentric factor due to the sensitivity of the logarithmic expressions to input information (Poling et al., 2001). The functional form of the acentric factor in CG method, Equation 3.10, can also be used with confidence. From Figure 3.5, although there is scatter in the data, the acentric factor does show an almost linear variation with carbon number. The prediction by Equation 3.10 is also qualitatively good and can be expected to give reasonable results when extrapolated. The parameters are also estimated with confidence in Table 3.5 .

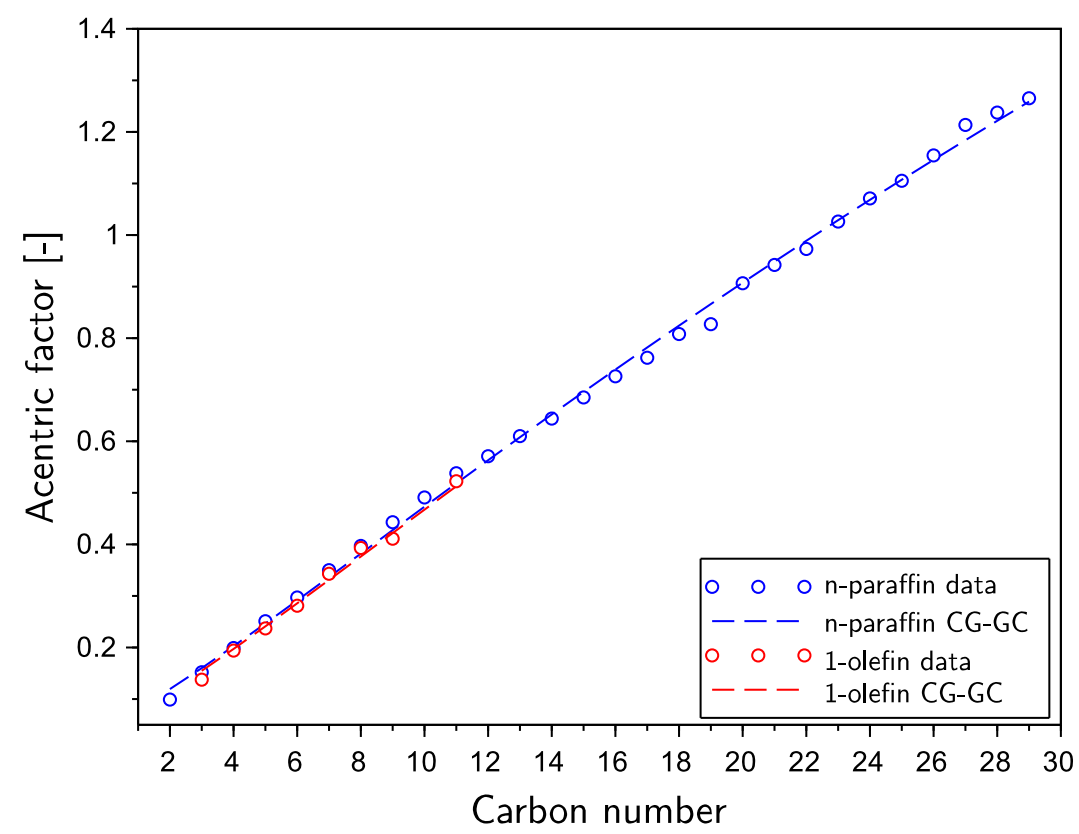

Figure 3.5: Acentric factor prediction by the CG method 


$$
\omega=0.4085\left(\ln \left(\sum_{k=1}^{n_{\text {groups }}} N_{k}\left(w_{1, k}\right)+1.1507\right)\right)^{\frac{1}{0.5050}}
$$

Table 3.5: Acentric factor parameters in Equation 3.10

\begin{tabular}{lc}
\hline Parameter & Value \\
\hline$W_{\mathrm{CH}_{3}}$ & $0.2803 \pm 0.0100$ \\
$W_{\mathrm{CH}_{2}}$ & $0.1530 \pm 0.0013$ \\
$W_{\mathrm{CH}}=\mathrm{CH}$ & $0.4143 \pm 0.0144$ \\
\hline
\end{tabular}

\subsubsection{Heat and Gibbs Free Energy of Formation Estimation}

The form of the function used by the CG method to estimate the ideal gas heat of formation and Gibbs free energy of formation is given by Equation 3.11 and 3.12 respectively. From Figures 3.6 and 3.7, the known linear dependence of the ideal gas formation properties on carbon number is accurately predicted by Equation 3.11 and 3.12. This means extrapolation can occur with a high degree of confidence, which is essential for the accurate estimation of equilibrium constants. The parameters required in Equation 3.11 and 3.12 are given in Table 3.6 .

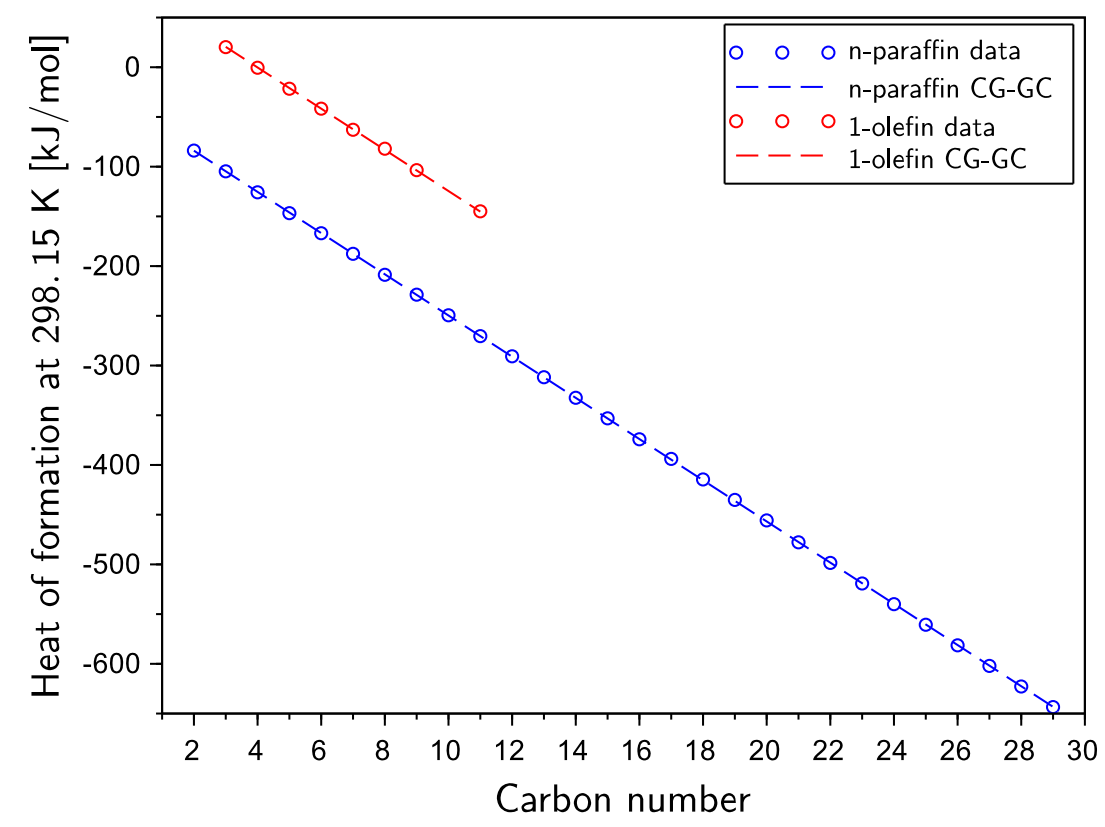

Figure 3.6: Heat of formation at $298.15 \mathrm{~K}$ prediction by the CG method 


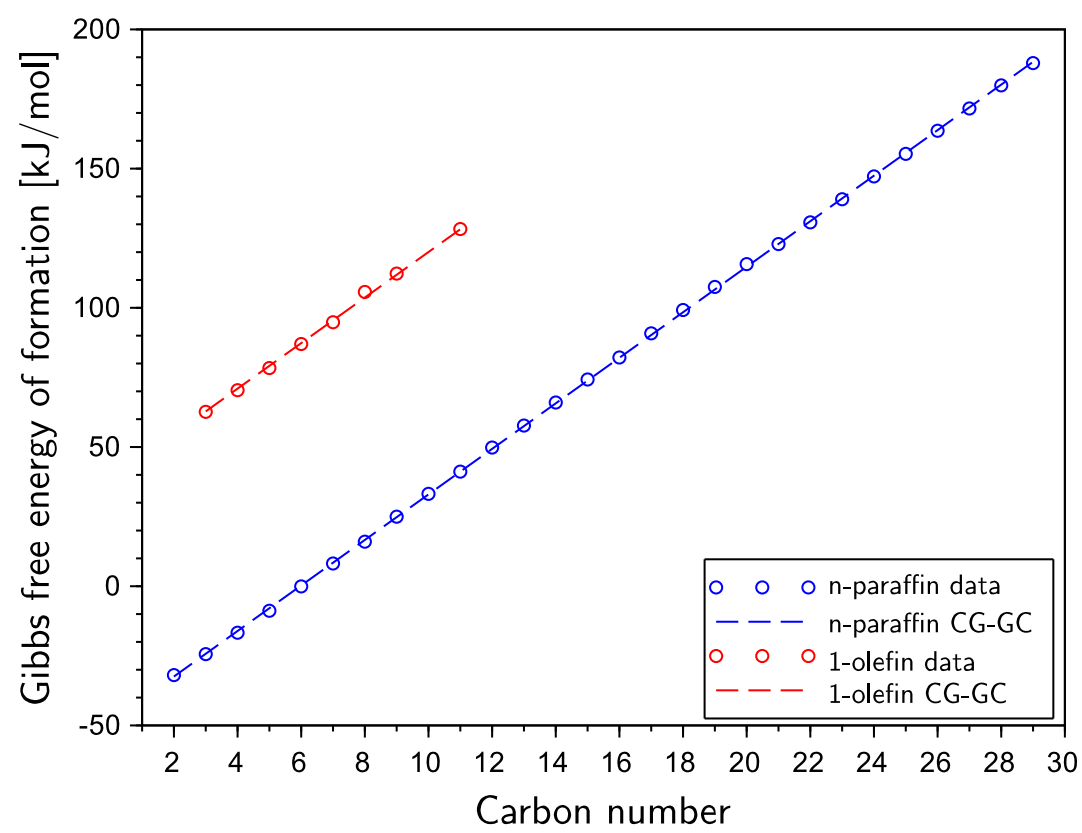

Figure 3.7: Gibbs free energy of formation at $298.15 \mathrm{~K}$ prediction by the CG method

$$
\begin{gathered}
\Delta H_{f}^{\circ}=10.835+\left(\sum_{k=1}^{n_{\text {groups }}} N_{k}\left(h f_{1, k}\right)\right) \\
\Delta G_{f}^{\circ}=-14.83+\left(\sum_{k=1}^{n_{\text {groups }}} N_{k}\left(g f_{1, k}\right)\right)
\end{gathered}
$$

Table 3.6: Ideal gas formation property parameters in Equation 3.11 and 3.12

\begin{tabular}{lc}
\hline Parameter & Value \\
\hline$h f_{\mathrm{CH}_{3}}[\mathrm{~kJ} / \mathrm{mol}]$ & $-47.39 \pm 0.08$ \\
$h f_{\mathrm{CH}_{2}}[\mathrm{~kJ} / \mathrm{mol}]$ & $-20.71 \pm 0.01$ \\
$h f_{\mathrm{CH}_{2}}=\mathrm{CH}[\mathrm{kJ} / \mathrm{mol}]$ & $57.05 \pm 0.21$ \\
$g f_{\mathrm{CH}_{3}}[\mathrm{~kJ} / \mathrm{mol}]$ & $-8.803 \pm 0.091$ \\
$g f_{\mathrm{CH}_{2}}[\mathrm{~kJ} / \mathrm{mol}]$ & $8.177 \pm 0.012$ \\
$g f_{\mathrm{CH}_{2}=\mathrm{CH}}[\mathrm{kJ} / \mathrm{mol}]$ & $86.43 \pm 0.32$ \\
\hline
\end{tabular}

\subsubsection{Ideal Gas Heat Capacity Estimation}

The form of the function, with a few modifications, used by the CG method to estimate ideal gas heat capacity is given in Equation 3.13. The reference temperature is chosen as $298.15 \mathrm{~K}$ instead of $700 \mathrm{~K}$ and the additional constants in the group contribution are dropped. The expression is 
quadratic in temperature because the temperature range under consideration is narrow (298.15$623.15 \mathrm{~K}$ ) and will provide a simpler formulation, that can be readily integrated to calculate enthalpy and Gibbs free energy changes.

$$
C_{p}=\left(\sum_{k=1}^{n_{\text {groups }}} N_{k}\left(C p a_{1, k}\right)\right)+\left(\sum_{k=1}^{n_{\text {groups }}} N_{k}\left(C p b_{1, k}\right)\right) \tau+\left(\sum_{k=1}^{n_{\text {groups }}} N_{k}\left(C p c_{1, k}\right)\right) \tau^{2}
$$

where

$$
\tau=\frac{T-298.15}{298.15}
$$

From Figure 3.8, Equation 3.13 is able to correlate the data well at all temperatures in the range considered. Furthermore, it also predicts the linear variation of the ideal gas heat capacity at constant temperature (Marano \& Holder, 1997c). The parameters which results from the regression are estimated with a high degree of confidence and are given in Table 3.7 .

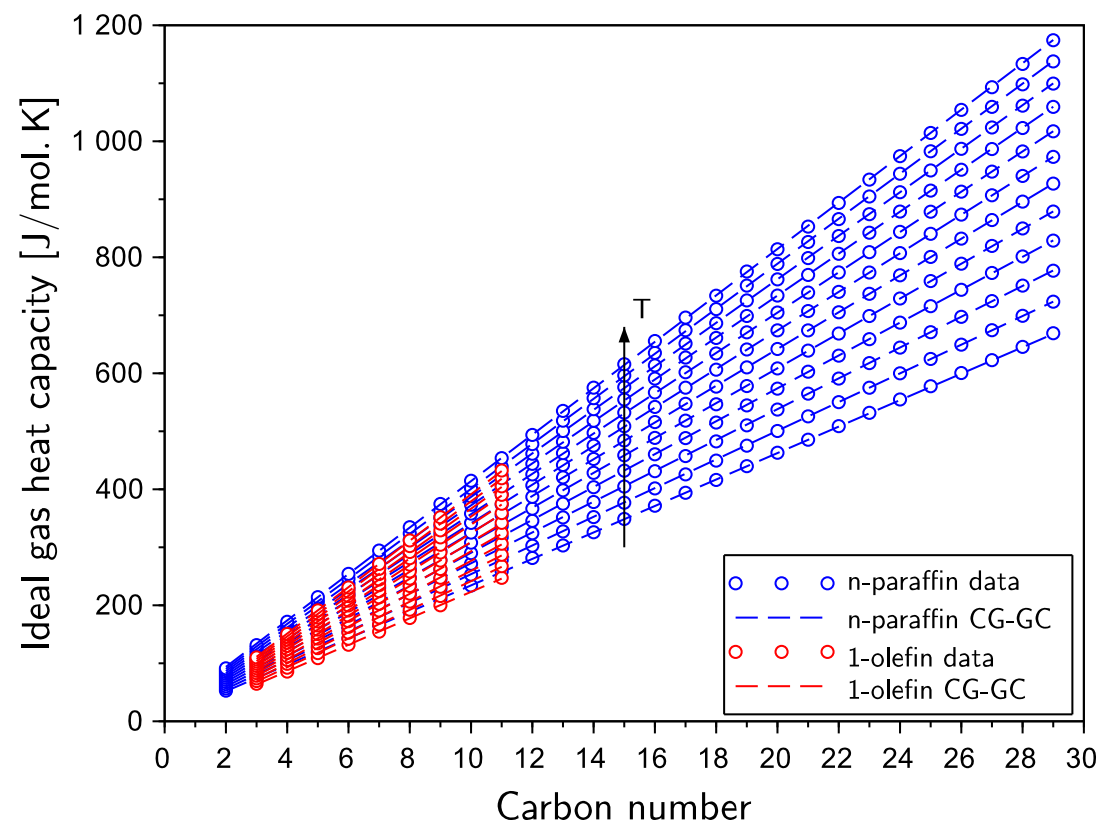

Figure 3.8: Ideal gas heat capacity prediction by the CG method. The black arrow indicates the direction of increasing temperature.

Table 3.7: Ideal gas heat capacity parameters in Equation 3.13

\begin{tabular}{lccc}
\hline Parameter & $\mathrm{a}$ & $\mathrm{b}$ & $\mathrm{c}$ \\
\hline $\mathrm{Cpi}_{\mathrm{CH}}[\mathrm{J} / \mathrm{mol} . \mathrm{K}]$ & $25.94 \pm 0.12$ & $20.52 \pm 0.53$ & $-1.147 \pm 0.466$ \\
$\mathrm{Cpi}_{\mathrm{CH}}[\mathrm{J} / \mathrm{mol} . \mathrm{K}]$ & $22.81 \pm 0.02$ & $19.66 \pm 0.07$ & $-3.546 \pm 0.059$ \\
$\mathrm{Cpi}_{\mathrm{CH}}=\mathrm{CH}[\mathrm{J} / \mathrm{mol} . \mathrm{K}]$ & $37.59 \pm 0.17$ & $30.86 \pm 0.72$ & $-5.830 \pm 0.638$ \\
\hline
\end{tabular}


The ideal gas heat capacity of the syngas components $\left(\mathrm{H}_{2}, \mathrm{CO}, \mathrm{CO}_{2}, \mathrm{H}_{2} \mathrm{O}\right.$ and $\left.\mathrm{N}_{2}\right)$ and methane and ethene can be calculated using Equation 3.14. The fit to data is shown in Figure 3.9, with the parameters that results from the regression given in Table 3.8 .

$$
C_{p}=a+b \tau+c \tau^{2}
$$

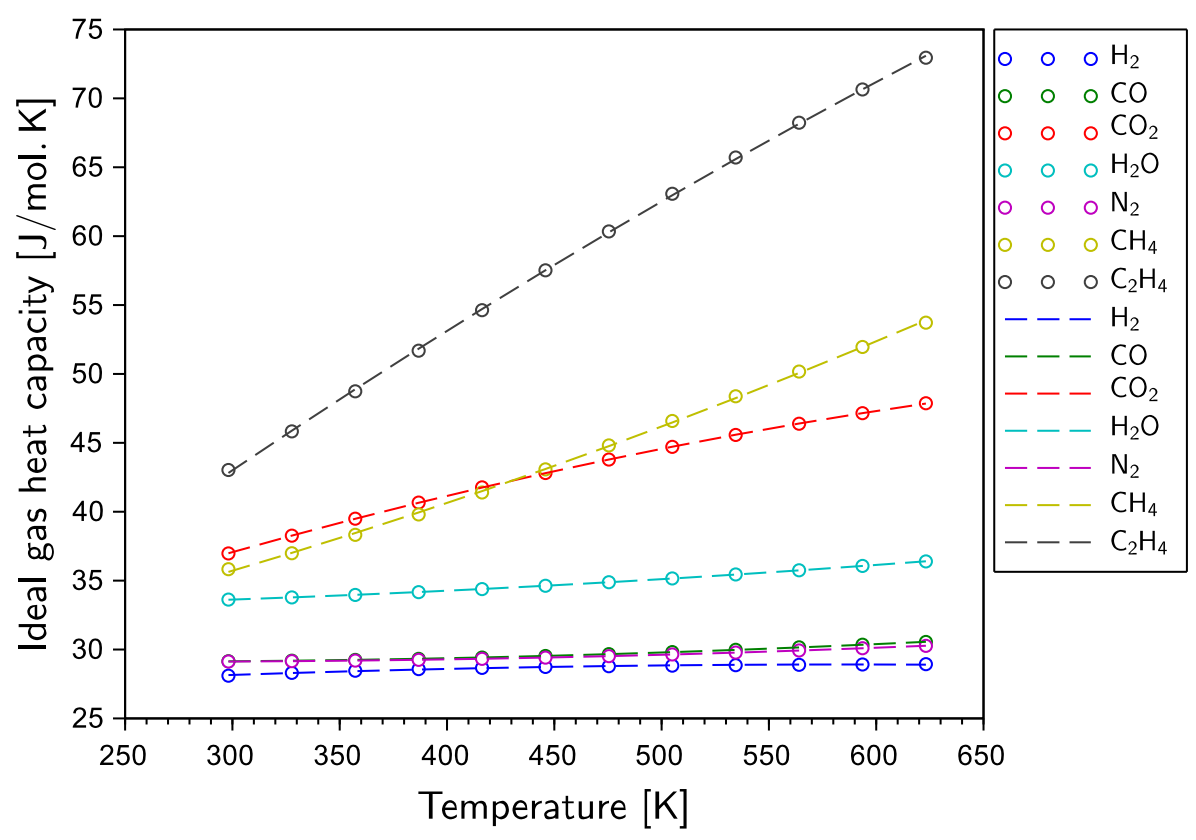

Figure 3.9: Syngas components, methane and ethene ideal gas heat capacity prediction.

Table 3.8: Syngas components, methane and ethene heat capacity parameters in Equation 3.14

\begin{tabular}{cccc}
\hline Chemical species & $\mathrm{a}[\mathrm{J} / \mathrm{mol} . \mathrm{K}]$ & $\mathrm{b}[\mathrm{J} / \mathrm{mol} . \mathrm{K}]$ & $\mathrm{c}[\mathrm{J} / \mathrm{mol} . \mathrm{K}]$ \\
\hline $\mathrm{H}_{2}$ & $28.15 \pm 0.00$ & $1.590 \pm 0.008$ & $-0.8226 \pm 0.0067$ \\
$\mathrm{CO}$ & $29.14 \pm 0.00$ & $0.3682 \pm 0.0004$ & $0.8545 \pm 0.0003$ \\
$\mathrm{CO}_{2}$ & $36.99 \pm 0.00$ & $13.21 \pm 0.00$ & $-2.972 \pm 0.001$ \\
$\mathrm{H}_{2} \mathrm{O}$ & $33.61 \pm 0.17$ & $1.620 \pm 0.001$ & $0.8622 \pm 0.0004$ \\
$\mathrm{~N} 2$ & $29.13 \pm 0.00$ & $0.1884 \pm 0.0001$ & $0.7910 \pm 0.0001$ \\
$\mathrm{CH} 4$ & $35.63 \pm 0.01$ & $13.68 \pm 0.18$ & $2.817 \pm 0.138$ \\
$\mathrm{C}_{2} \mathrm{H}_{4}$ & $42.81 \pm 0.01$ & $31.28 \pm 0.17$ & $-3.221 \pm 0.133$ \\
\hline
\end{tabular}




\subsection{Thermodynamic Modelling}

The most important decision when simulating a process is the selection of an appropriate thermodynamic model. This ensures that the behaviour of the real fluid is accurately approximated during energy balance and chemical and phase equilibrium calculations. The safe choice of a thermodynamic model requires knowledge of the system, available calculation options and the margin of error introduced in using the model (Turton et al., 2009).

Under the LTFT reaction conditions of $180-260^{\circ} \mathrm{C}$ and $20-30$ bar(a), the reaction mixture distributes between the vapour and liquid phases respectively (Marano \& Holder, 1997d; Visconti, 2014). The chemical species present are $\mathrm{H}_{2}, \mathrm{CO}, \mathrm{CO}_{2}, \mathrm{H}_{2} \mathrm{O}$ and mainly linear 1-olefins of low carbon number and $n$-paraffins of high carbon number. The reaction mixture in either phase therefore consists of both slightly polar and non-polar chemical species in both the subcritical and supercritical regions.

Either Henry's law or an equation of state (EOS) are suitable choices for calculating the VLE of this system (Turton et al., 2009). The use of Henry's law is a simple, empirical method. Henry's constant, which determines liquid solubility, is dependent on pressure, temperature and the solvent type (Elliot \& Lira, 2012). This restricts its predictive ability because it cannot be assumed to remain valid outside the range of experimental conditions under which it was measured. While it is acknowledged that both the vapour and liquid phases may exhibit ideal behaviour, the presence of supercritical components and many chemical species with molecules of varying size necessitates the need for an EOS.

The Peng-Robinson (PR)-EOS has most commonly been used for this system e.g. in the work of Marano \& Holder (1997a), Ahón et al. (2005), Derevich et al. (2008), Mthombeni (2009), Visconti (2014) and Khazali (2018). This is because it is well-suited to VLE calculations of systems with non-polar and slightly polar compounds and is superior in terms of predictive ability relative to other EOS of similar simplicity, specifically the van der Waals (vdW) and Soave-Redlich-Kwong (SRK) EOS (Twu et al., 1995).

The PR-EOS is an algebraic equation for pressure $P$ as a function of temperature $T$, molar volume $\underline{V}$ and composition $z_{i}$ (Turton et al., 2009). In Equation 3.15, the parameters $a$ and $b$ represent 'attraction' and 'repulsion' respectively, based on van der Waals formulation (Elliot \& Lira, 2012). The prediction of condensation and hence the liquid phase is determined by the alpha function contribution $\alpha(T, \omega)$ to the pure component attractive term in Equation 3.16 . The alpha function is a temperature and acentric factor dependent expression which is used to correlate the pure component vapour pressure (Twu et al., 1995). The inclusion of the acentric factor $\omega$ provides a 
useful experimental vapour pressure which improves the predictive capability of the EOS (Elliot \& Lira, 2012). For mixtures, the parameters $a$ and $b$ are evaluated using an appropriate mixing rule. The ability of the PR-EOS to describe the VLE of the FT synthesis reaction mixture therefore depends on the choice of mixing rule and the alpha function used to represent the pure component vapour pressure.

$$
\begin{gathered}
P=\frac{R T}{\underline{V}-b}-\frac{a\left(T, \omega, z_{i}\right)}{\underline{V}^{2}-2 b\left(z_{i}\right) \underline{V}-b^{2}\left(z_{i}\right)} \\
a_{i}=0.4572 \frac{\left(R T_{c}\right)^{2}}{P_{c}} ; \quad b_{i}=0.0778 \frac{R T_{c}}{P_{c}}
\end{gathered}
$$

\subsubsection{Choice of Mixing Rule}

The most convenient approach for extending the PR-EOS to mixtures is to make the parameters, $a$ and $b$, vary with composition. There is no theoretical basis for this, but it is useful for bringing together pairwise the interactions between chemical species (Poling et al., 2001). This results in the mixture properties and their variation with temperature and pressure, at fixed composition, being the same as for some pure component i.e. the concept of a 'one fluid' (Poling et al., 2001). Most process simulators make use of the van der Waals one fluid mixing rule, where $a$ and $b$ have a quadratic dependence on the mole fractions of each component in each phase, given by Equation 3.17. Here, $a_{i} / a_{j}$ and $b_{i} / b_{j}$ are the pure component attractive and repulsive parameters and $p_{i} / p_{j}$ refers to the mole fraction of species $i / j$ in either the vapour or liquid phase.

$$
a=\sum_{i=1}^{n} \sum_{i=1}^{n} p_{i} p_{j} \sqrt{a_{i j}}\left(1-k_{i j}\right) ; \quad b=\sum_{i=1}^{n} p_{i} b_{i}
$$

The inclusion of the binary interaction parameter (BIP), $k_{i j}$, improves the prediction of VLE by the PR-EOS (Turton et al., 2009). BIPs are estimated from experimental VLE data. This requires experimental data for each binary pair in a multi-component mixture, which is not readily available for the FT reaction system. To overcome this, various group contribution methods (GCM(s)) exist to estimate BIPs (Turton et al., 2009; Privat \& Jaubert, 2012). While these are useful, these GCMs only apply to specific mixtures and are dependent on the EOS and alpha function used to develop them. The empirical nature of these GCMs also makes them poorly suited to extrapolation (Privat \& Jaubert, 2012).

It should be noted that the availability of BIPs is not indicative of model accuracy (Turton et al., 2009). The BIPs themselves are empirical parameters representing the result of a best fit to experimental data. In the absence of these data, it is acceptable to set the value of the BIP to zero (Turton et al., 2009). While this is a decision to use a specific value of the BIPs, the 
margin of error introduced is more conservative than using a non-zero value from some estimation method which might lead to erroneous results. The FT reaction mixture also consists of mainly non-polar and slightly polar molecules and can be regarded as a normal fluid, whose behaviour could be approximated without needing BIPs (Poling et al., 2001). The work of Marano \& Holder (1997a) showed that the inclusion of BIPs had little to no effect on the calculation of fugacity coefficients, while the work of Le Grange (2009) showed that there is almost no difference between the calculated VLE as a function of carbon number with and without BIPs. Reasonably accurate results can be expected using the van der Waals mixing rule, without BIPs, because of the nature of the chemical species and intermolecular forces present (Privat \& Jaubert, 2012).

\subsubsection{Choice of Alpha Function}

The choice of alpha function in the PR-EOS also influences its prediction of the VLE of mixture since it represents the pure component vapour pressure (Twu et al., 1995). This is important since pure component properties determine most of the observed behaviour of mixtures (Poling et al., 2001). The most common form of the alpha function, generalised by the Law of Corresponding States 2 , is that of Soave in Equation 3.18 as a function of reduced temperature $T_{r}$.

$$
\alpha(T, \omega)=\left(1+m\left(1-\sqrt{T_{r}}\right)\right)^{2}
$$

The use of Equation 3.18 in the PR-EOS of 1976 allowed it to become one of the most commonly applied to the VLE of systems containing non-polar and slightly polar components (Twu et al., 1995). This is due to the generalisation of $m$ in terms of the acentric factor given by Equation 3.19.

$$
m=0.37464+1.54226 \omega-0.26992 \omega^{2}
$$

The alpha function is then a $4^{\text {th }}$ order polynomial in the acentric factor. Equation 3.19 performs well for light hydrocarbons at $0.7 \leq T_{r} \leq 1$, but it is only applicable up to $\omega=0.491$ (n-decane), with significant errors in the vapour pressure prediction of components with larger acentric factors (Twu et al., 1995). Errors increase rapidly for all compounds at low reduced temperatures. This means its behaviour cannot be valid when extrapolated to heavy hydrocarbons i.e. for $\omega>0.491$ (Twu et al., 1995). This was recognised and as such the PR-EOS of 1978 incorporated a second polynomial expression, Equation 3.20, for $\omega>0.491$. The use of a higher order polynomial improved vapour pressure predictions in the reduced temperature range $0.7 \leq T_{r} \leq 1$. However, this form of the alpha function still presents a problem for extrapolation because of the behaviour of

\footnotetext{
${ }^{2}$ The Law of Corresponding States asserts that all fluid properties are similar and represented by the same function if properly expressed in terms of reduced pressure, temperature and volume (Poling et al. 2001 Elliot \& Lira |2012).
} 
the polynomial expression outside of the range of acentric factors used to develop the correlation. Twu et al. (1995) indicates that outside this range of reduced temperatures, there are not just errors for heavy hydrocarbons, but all compounds this EOS could be applied to.

$$
m=0.379642+1.487503 \omega-0.164423 \omega^{2}+0.016666 \omega^{3}
$$

It is evident that the extrapolation of the alpha function to describe heavy hydrocarbons is limited it by being expressed as a polynomial function of the acentric factor. It is important to note that a temperature-dependent alpha function must satisfy the following (Twu et al., 1991):

1. It must be finite and positive for all temperatures.

2. It must approach a finite value as the temperature approaches infinity.

3. It must have a value of unity at the critical temperature.

4. All of the above must be preserved for all meaningful values of $\omega$.

Twu et al. (1995) took a different approach to the one presented by Soave by expanding the alpha function as a power series in the acentric factor as shown by Equation 3.21. Here, $\alpha_{0}$ and $\alpha_{1}$ correspond to $\omega=0$ and $\omega=1$ respectively and are functions of $T_{r}$. A clear advantage of Equation 3.21 is that it is a linear function in $\omega$, allowing it to be extrapolated to heavy hydrocarbons (Twu et al. 1995). The temperature dependence of the alpha function in reduced form is given by Equation 3.22

$$
\begin{gathered}
\alpha\left(T_{r}, \omega\right)=\alpha_{0}+\omega\left(\alpha_{1}-\alpha_{0}\right) \\
\alpha_{i}\left(T_{r}\right)=T_{r}^{N_{i}\left(M_{i}-1\right)} \exp \left(L_{i}\left(1-T_{r}^{N_{i} M_{i}}\right)\right)
\end{gathered}
$$

While Equation 3.22 solves the problem of extrapolation at reduced temperatures $T_{r} \geq 0.5$ and extrapolation to large $\omega$, it remains essential to address the alpha function predictions of chemical species above their critical temperature. For this purpose, hydrogen and methane, chemical species which are beyond their critical temperature for most applications, were used to develop separate alpha expressions (Twu et al., 1995). The result is two alpha functions, one for the subcritical region and another for the supercritical region. While developed based on a limited range of compounds, the generality based on the Law of Corresponding States and functional formulation proposed by Twu et al. (1995) can safely be assumed to hold for a wide range of $\omega$ and $T_{r}$.

It is more informative to compare the performance of the alpha functions used in the PR-EOS of 1976, 1978 and the alpha function developed by Twu et al. (1995) at a FT reactor temperature 
of $220^{\circ} \mathrm{C}$ for the n-paraffins in Figure 3.10. The different alpha function formulations produce the same behaviour up to $C_{20}$, possibly because this only results in a mild extrapolation of the polynomial expression at this stage. Thereafter, the alpha function of PR-EOS of 1976 deviates, approaching a maximum around $C_{70}$ before decreasing. This is qualitatively incorrect (Le Grange, 2009), as species of high carbon number are high boiling which implies high alpha values. The difference between the alpha functions used in the PR-EOS of 1978 and Twu et al. (1995) et al. (1995) becomes apparent around $C_{80}$, where the behaviour of the polynomial expression causes the alpha values to increase more rapidly indicating that extrapolation is questionable at very high carbon numbers. In contrast, the Twu et al. (1995) alpha function extrapolation to high carbon number is more well-behaved. This is shown by the steadier rise in alpha value which represents a more conservative prediction of the vapour pressure at high carbon numbers in Figure 3.10. In fact, it appears that it is approaching a limiting value which is consistent with the limiting behaviour of the vapour pressure within the n-paraffin homologous series (Marano \& Holder, 1997b). From this analysis, it can be concluded that the Twu et al. (1995) alpha function is more suitable to represent heavy chemical species and those in the supercritical region. It is therefore well-suited to represent VLE behaviour in FT synthesis.

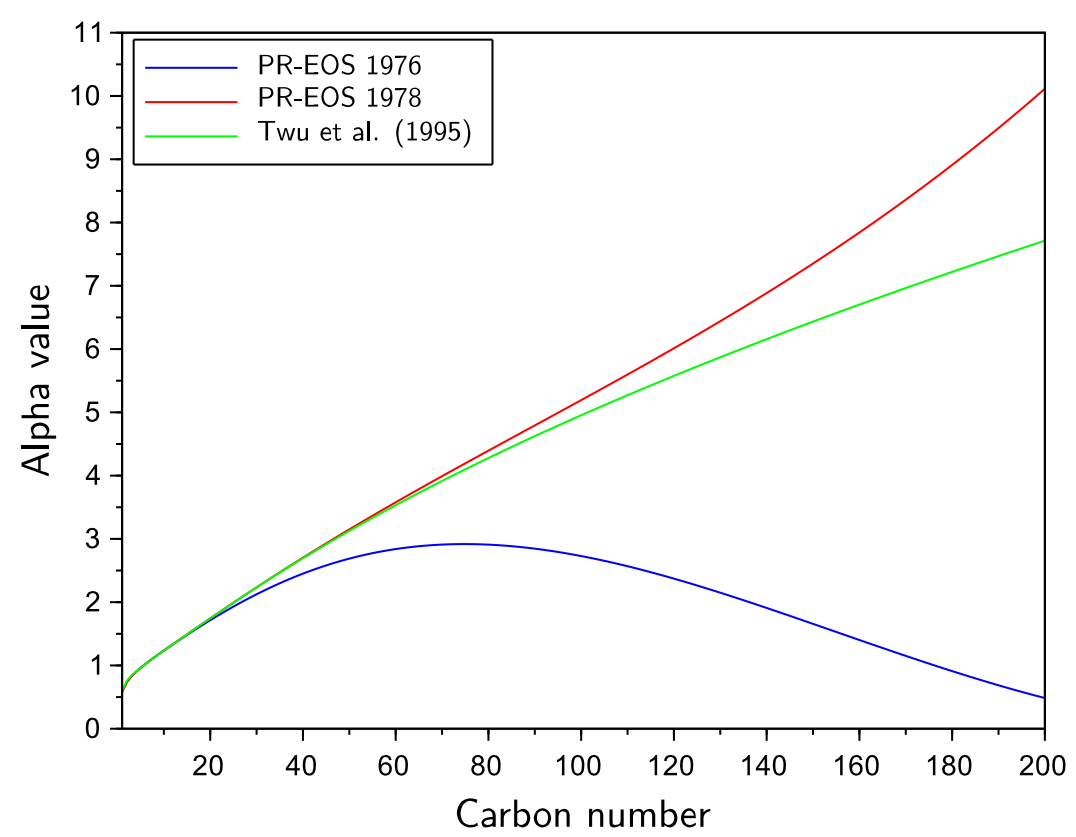

Figure 3.10: Comparison of the different alpha functions used in the PR-EOS applied to the n-paraffin homologous series adapted from Le Grange (2009) 


\section{Chapter 4}

\section{Model Development}

The description of FT reaction kinetics from first principles is necessary for reactor modelling and design. Most kinetic models in literature use Lanmguir-Hinshelwood (LH) theory, based on the alkyl mechanism, to develop rate expressions for n-paraffin and 1-olefin formation (van der Laan, 1999, Visconti et al., 2007). This requires the proposal of rate-determining steps (RDS), commonly taken as the termination or product formation steps. Complex rate expressions result which require many adjustable parameters to describe FT reaction behaviour (Norval, 2008). The allocation of RDS and the form of these rate expressions mean that the polymerisation character of the reaction is lost and the relative contribution of each reaction step is not captured in its entirety (Allie \& Nyathi, 2019). Examples are the models of Todic et al. (2014), Sage et al. (2014), Kamal K. Pant \& Upadhyayula (2019) and Marchese et al. (2019). Apart from the model of Marchese et al. (2019), the other models mentioned are only valid up to carbon number $C_{15}$ or $C_{20}$.

The failure of these models to account for a sizeable fraction of the product distribution makes estimation of the quantities of high carbon number products questionable. As such, it is unclear whether these models will remain reliable when extrapolated to high carbon numbers. It is known that the kinetic parameters in LH type models exhibit a high degree of correlation (Schwan, 2001; Sharrock \& Coetzer, 2007; Hillestad et al., 2016). This complicates the parameter estimation process, which coupled with the inability to accurately represent the product distribution reduces the confidence in the kinetic parameter estimate extracted from experimental data.

It appears that only the models of Visconti et al. (2007) and Visconti et al. (2011) preserve the polymerisation character of the FT reaction. These models avoid proposing RDS and simulate each elementary step of adsorption, surface reaction and desorption. While this ensures that the relative contribution of each reaction step to the overall reaction rate is captured, a highly implicit, 
complex model results which cannot easily be applied and used. Furthermore, all models in literature are reliant on various assumptions such as the carbon number dependence of 1-olefin secondary reactions to account for the observed characteristics of the FT product distribution, despite the reasons behind them remaining unknown (Puskas \& Hurlbut, 2003). While this reiterates that FT reaction behaviour cannot be described by kinetics alone (Glasser et al., 2012), it is clear that a new approach to FT kinetic modelling is required.

\subsection{Partial Equilibrium Approach to Kinetic Modelling}

The development of a robust, broadly applicable and simple kinetic model is the key to describing FT reaction behaviour. A useful approach which is commonly used to reduce detailed reaction mechanisms in fuel combustion is the assumption of partial equilibrium (Fry, 2010). The existence of partial equilibrium in the FT reaction system was first demonstrated in the work of Norval \& Phillips (1990) and Norval (2008), in which the ASF distribution was shown to have a thermodynamic basis. The n-paraffin and 1-olefin homologous series are therefore separate subsystems working to achieve partial equilibrium. This indicates that reaction kinetics controls the yield of these hydrocarbons and that thermodynamics controls their distribution. Thus, the chain growth reaction steps must be fast relative to the formation of the initial hydrocarbon species (Norval \& Phillips, 1990).

A micro-kinetic modelling study was conducted by Hensen et al. (2020) on a Co-based catalyst as an attempt to simulate $\mathrm{CO}$ hydrogenation towards methane. The initiation and chain growth steps were assumed to occur as given by the alkyl mechanism. It is known that the CO bond must be broken before carbon-carbon coupling during chain growth can occur. It was shown by Hensen et al. (2020) that CO bond breakage, followed by the hydrogenation of $C$ and $O$, is thus rate-determining. Allie \& Nyathi (2019) performed a micro-kinetic comparison of CO and $\mathrm{CO}_{2}$ hydrogenation towards methane and $\mathrm{C}_{2}$ hydrocarbons on an $\mathrm{Fe}(100)$ surface. A sensitivity analysis was performed to determine the degree of rate control and the results indicated that $\mathrm{CO}$ bond breakage is rate-determining in the formation of $C_{1}$ and $C_{2}$ hydrocarbons. This is since $C O$ bond breakage affects the availability of free surface carbon and hence the rate at which carbon is hydrogenated and coupled into hydrocarbons (Allie \& Nyathi, 2019). These findings were reported to agree with the work of van Helden et al. (2017) for CO hydrogenation on Co.

Mims et al. (1990) co-fed 1-hexene and 1-octene over Ru-based catalysts. They concluded that these 1-olefins polymerise and that the rate of chain growth is greater than the rate of initiation. This is often also the case in free radical polymerisation by the long-chain approximation ${ }^{1}$

\footnotetext{
${ }^{1}$ In free radical polymerisation, the long-chain approximation allows the rate of initiation to be neglected when
} 
(Fogler, 1999). A semi-logarithmic plot is obtained, consistent with equilibration within respective homologous series (Norval, 2008). This indicates that chain growth reaction rates are rapid enough to achieve partial equilibrium among a subset of species i.e. within a homologous series but not complete chemical equilibrium among all species (Norval, 2008). During kinetic measurements, it is observed that $\mathrm{CO}$ is the dominant adsorbed species, with a low coverage of growing chains (Yamasaki et al., 1981; Kobori et al., 1982; Todic et al., 2014). This is consistent with the slow hydrogenation of $\mathrm{CO}$ and partial chemical equilibrium being a reasonable explanation for the product distribution (Norval, 2008). It is therefore important to consider the implications of partial equilibrium before attributing observations to more traditional kinetic effects and building a complex kinetic model.

$\mathrm{NH}_{3}$ synthesis, $\mathrm{SO}_{2}$ oxidation and propene oligomerisation are examples of equilibrium-controlled systems that can be effectively modelled using homogeneous reactions (Sealy, 1996; Norval, 2008). The reaction rate in these systems can be effectively correlated using a rate expression in the form of Equation 4.1. where df represents the kinetic driving force and $\mathrm{K}_{\mathrm{df}}$ represents the equilibrium constant based on the kinetic driving force used.

$$
r=(\text { kinetic term })\left(d f_{\text {reactants }}-\frac{d f_{\text {products }}}{K_{d f}}\right)
$$

This means that the reaction rate can be effectively correlated without including the effect of reactant or product adsorption. By considering the reactions involved in FT synthesis as equilibriumcontrolled, the kinetic model formulation could be significantly simplified and consist of fewer rate expressions. The number of kinetic parameters that would be required could also be reduced without compromising on prediction quality or thermodynamic consistency.

\subsection{Model Formulation}

It is clear that a model describing FT kinetics must satisfy the following criteria:

1. It must preserve the polymerisation character of the FT reactions to ensure that the product distribution of LTFT synthesis is adequately represented. This will later allow reliable estimates of kinetic parameters to be extracted from experimental data.

2. It must consider the partial chemical equilibrium aspects that exist in the n-paraffin and 1olefin homologous series that give rise to the thermodynamic basis of the ASF distribution (Norval \& Phillips, 1990; Norval, 2008).

describing the net rate of monomer consumption. This is valid when the rate of chain growth is greater than the rate of initiation and the ratio of monomer to initiator concentration is high (Fogler. 1999). 
3. It must be thermodynamically consistent once complete chemical equilibrium is established.

There is general agreement that FT synthesis may be viewed as a methylene polymerisation reaction (Fernandes, 2006). Methylene is the monomer unit produced from the hydrogenation of CO. With this in mind and to satisfy the above criteria, the reaction pathway in Figure 4.1 is proposed.

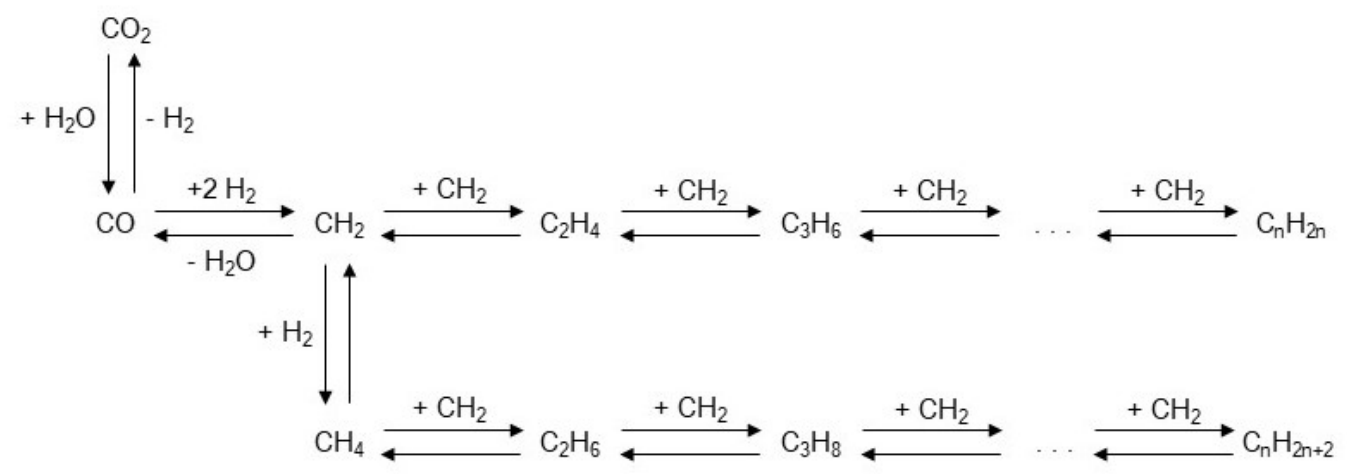

Figure 4.1: Proposed FT reaction pathway adapted from Fernandes (2005) and Schulz et al. (1994)

The first step in Figure 4.1 involves the hydrogenation of $\mathrm{CO}$ to form methylene $\left(\mathrm{CH}_{2}\right)$ and water. Here, $\mathrm{CH}_{2}$ is a pseudo-species introduced by Norval (2008) to describe the FT system using an equilibrium approach. Alternatively, CO may also react with water in the WGS reaction to form $\mathrm{CO}_{2}$ and $\mathrm{H}_{2}$. Methylene is then directly hydrogenated to form methane (Patzlaff et al., 1999. Subiranas, 2009). Two separate chain growth reactions are proposed, one for n-paraffins and another for 1-olefins using a series-parallel reaction network (Mthombeni, 2009). From Figure 4.1. carbon-carbon coupling, which facilitates chain growth, occurs through the addition of $\mathrm{CH}_{2}$. The reaction pathway proposed in this way ensures that the n-paraffin and 1-olefin homologous series are treated as separate subsystems working to achieve partial chemical equilibrium (Norval \& Phillips, 1990, Norval, 2008). The set of reactions involved in Figure 4.1 are summarised in Table 4.1 .

Table 4.1: Chemical reactions in the proposed reaction pathway

\begin{tabular}{lc}
\hline Reaction step & Chemical reaction \\
\hline 1. Monomer formation & $\mathrm{CO}+2 \mathrm{H}_{2} \rightleftharpoons \mathrm{CH}_{2}+\mathrm{H}_{2} \mathrm{O}$ \\
2. Methanation & $\mathrm{CH}_{2}+\mathrm{H}_{2} \rightleftharpoons \mathrm{CH}_{4}$ \\
3. Initiation $(n=1)$ & $\mathrm{CH}_{2}+\mathrm{CH}_{4} \rightleftharpoons \mathrm{C}_{2} \mathrm{H}_{6}$ \\
4. n-Paraffin chain growth $(n=2,3, \ldots, \mathrm{N}-1)$ & $\mathrm{CH}_{2}+\mathrm{C}_{n} \mathrm{H}_{2 n+2} \rightleftharpoons \mathrm{C}_{n+1} \mathrm{H}_{2(n+1)+2}$ \\
5. 1-Olefin chain growth $(n=1,2, \ldots, \mathrm{N}-1)$ & $\mathrm{CH}_{2}+\mathrm{C}_{n} \mathrm{H}_{2 n} \rightleftharpoons \mathrm{C}_{n+1} \mathrm{H}_{2(n+1)}$ \\
6. Water gas shift (WGS) & $\mathrm{CO}+\mathrm{H}_{2} \mathrm{O} \rightleftharpoons \mathrm{H}_{2}+\mathrm{CO}_{2}$ \\
\hline
\end{tabular}


In Table 4.1, $N$ represents the highest carbon number present in the FT reaction system and the specified truncation point that adequately represents a sizeable fraction of the product distribution. It is noted, based on the work of Khazali (2018), that the cut-off point should be extended to $N+1$. This prevents $N$ from acting as a barrier in the chain growth reactions. Thus, the error in the estimation of the products with carbon number $N$ is mitigated (Khazali, 2018). The rate expression of each reaction step $1-6$ is given in Table 4.2 .

Table 4.2: Rate expressions for the reaction steps in the proposed reaction pathway

\begin{tabular}{lc}
\hline Reaction step & Rate expression \\
\hline 1. Monomer formation & $r_{1}=k_{1}\left(a_{C O} a_{H_{2}}^{2}-\frac{a_{C H_{2}} a_{H_{2} O}}{K_{a, 1}}\right)$ \\
2. Methanation & $r_{2}=k_{2}\left(a_{\left.C H_{2} a_{H_{2}}-\frac{a_{C H_{4}}}{K_{a, 2}}\right)}\right.$ \\
3. Initiation $(n=1)$ & $r_{3}=k_{3}\left(a_{C H_{2}} a_{C H_{4}}-\frac{a_{C 2} H_{6}}{K_{a, 3}}\right)$ \\
4. n-Paraffin chain growth $(n=2,3, \ldots, N-1)$ & $r_{n+2}=k_{4}\left(a_{C H_{2}} a_{C_{n} H_{2 n+2}}-\frac{a_{C n+1} H_{2(n+1)+2}}{K_{a, n+2}}\right)$ \\
5. 1-Olefin chain growth $(n=1,2, \ldots, N-1)$ & $r_{N+n+1}=k_{5}\left(a_{C H_{2}} a_{C_{n} H_{2 n}}-\frac{a_{C_{n+1} H_{2(n+1)}}}{K_{a, N+n+1}}\right)$ \\
6. Water gas shift (WGS) & $r_{2 N+1}=k_{6}\left(a_{C O} a_{H_{2} O}-\frac{a_{H_{2} a} a_{C O}}{K_{a, 2 N+1}}\right)$ \\
\hline
\end{tabular}

By assuming that the FT reaction system consists of a number of subsystems working to achieve partial equilibrium, each reaction rate can be formulated as an equilibrium-controlled process in the form of Equation 4.1 (Norval, 2008). The kinetic driving force in each rate expression in Table 4.2 is the species activity in Equation 4.2. where $z_{i}^{\text {phase }}$ is the mole fraction of species $i$ in a particular phase, $\phi_{i}^{\text {phase }}$ is the phase fugacity coefficient and $P_{\text {total }}$ and $P_{\text {ref }}$ are the total reactor and reference pressures respectively. The fugacity coefficient in a particular phase is evaluated using the PR-EOS.

$$
a_{i}=\frac{z_{i}^{\text {phase }} \phi_{i}^{\text {phase }} P_{\text {total }}}{P_{\text {ref }}}
$$

Since the calculation of the equilibrium constant $K_{a, n}$ uses the ideal gas as the reference state, the reference fugacity simplifies to the reference pressure, which is commonly 1 bar(a). The choice of the species activity as the kinetic driving force ensures that $K_{a, n}$ is a function of temperature only and will later allow the effect of VLE on the kinetic behaviour to be easily incorporated (Laxmi Narasimhan et al., 2006; Mthombeni, 2009; Elliot \& Lira, 2012).

The rate expressions in Table 4.2 are all elementary apart from monomer formation, reaction step 1. The form of the rate expression in reaction step 1 allows the many initial steps that lead to $\mathrm{CH}_{2}$ (monomer) formation to be represented in a compact manner. These initial steps remain debated 
in literature (Mousavi et al., 2015), and as such the form of the rate expression used here avoids these controversies while still effectively correlating the rate of $\mathrm{CO}$ consumption and capturing the high exothermicity of the CO hydrogenation (Subiranas, 2009). Furthermore, the form of the rate expressions in Table 4.2 retains the polymerisation character of the FT reaction and ensures that chain growth occurs through the addition of $\mathrm{CH}_{2}$ as given by the alkyl mechanism (Sie et al., 1991; van der Laan, 1999; Lozano-Blanco et al., 2006). The model therefore satisfies Criterion 1.

The hydrogenation of $\mathrm{CO}$ to $\mathrm{CH}_{2}$ has been shown to be rate-determining and enables the introduction of the pseudo-species $\mathrm{CH}_{2}$ (van Helden et al., 2017; Allie \& Nyathi, 2019; Hensen et al., 2020). The major advantage of introducing the pseudo-species $\mathrm{CH}_{2}$ is that it allows the $\mathrm{FT}$ reactions to be represented by an equilibrium approach (Norval, 2008). The thermodynamic basis of the ASF distribution is based on the linear variation of the ideal gas Gibbs free energy of formation with carbon number in the n-paraffin and 1-olefin homologous series (Norval \& Phillips, 1990). This is confirmed by the results in Chapter 3. The $\mathrm{CH}_{2}$ functional group is responsible for the linear variation of the ideal gas Gibbs free energy of formation of n-paraffins and 1-olefins with carbon number. The Gibbs free energy of $\mathrm{CH}_{2}$ can therefore be easily estimated from the gradient of the linear relationship with carbon number at constant temperature. The assumptions made in Chapter 3 also ensures that the value of the Gibbs free energy of $\mathrm{CH}_{2}$ is the same for $\mathrm{n}$-paraffins and 1-olefins. All equilibrium constants $K_{a, n}$ can therefore be estimated using Equation 4.3. Together with the separate chain growth reactions for n-paraffin and 1-olefin formation in Table 4.1, this ensures that kinetic model satisfies Criterion 2.

$$
K_{a, n}=\exp \left(-\frac{\Delta G_{r \times n, n}^{\circ}}{R T}\right)
$$

All rate constants in the model are independent of carbon number. The exception is $k_{3}$ which functions as an initiation rate constant. This accounts for the low chain growth probability and reactivity of $\mathrm{CH}_{4}$ towards higher n-paraffins (see Schulz \& Claeys (1999)). The chain growth reaction steps 4 and 5 in Table 4.1 indicates that n-paraffins and 1-olefins of carbon number $C_{2}$ and greater proceed along the same mechanistic pathway. The number of unknown rate constant is then reduced, one for each family of reaction steps (Möller et al., 2009). This ensures that the observed carbon number dependence in reactivity arises from the equilibrium position of the reactions in reaction step 4 and 5 .

A key assumption made in the formulation of the kinetic model is that the net rate of formation of pseudo-species $\mathrm{CH}_{2}$ is equal to zero, which can easily be shown. The rate of consumption of $\mathrm{CO}$ by the $\mathrm{FT}$ reactions only, on a carbon basis, is equal to the rate of formation of organic compounds, 
namely n-paraffins and 1-olefins, given by Equation 4.4 (Claeys \& van Steen, 2004).

$$
-r_{C O}^{F T}=r_{C, o r g}
$$

In the proposed $\mathrm{FT}$ reaction pathway, the rate of $\mathrm{CO}$ consumption is equivalent to the rate of reaction step 1 which leads to $\mathrm{CH}_{2}$ formation. The rate of $\mathrm{CH}_{2}$ formation is then equivalent to the rate of formation of organic compounds on a carbon basis in Equation 4.5 .

$$
r_{1, \mathrm{CH}_{2}}=r_{\mathrm{C}, \mathrm{org}}
$$

The result of the equivalence in Equation 4.5 is that the net rate for the pseudo-species $\mathrm{CH}_{2}$ is zerd 2 . This means that the slow rate of $\mathrm{CH}_{2}$ formation in reaction step 1 is compensated by the fast rate of $\mathrm{CH}_{2}$ consumption in reaction steps 2-5. Consequently, this results in a high reactivity of the pseudo-species $\mathrm{CH}_{2}(\overline{\text { Fogler, }}$ 1999). The pseudo-steady state approximation can therefore be safely applied. This is validated in Appendix A.1.

On a carbon basis, the rate of organic compound formation is equivalent to the sum of the rate of $\mathrm{CH}_{2}$ consumption in the methanation, initiation and n-paraffin and 1-olefin chain growth reactions in Equation 4.6. This can be expanded and expressed in terms of species activity by substituting in each rate expression for reaction step 1-5 given in Table 4.2. The result is a quadratic expression, Equation 4.7, in the activity of $\mathrm{CH}_{2}$.

$$
\begin{array}{r}
r_{1, C H_{2}}-r_{C, \text { org }}=r_{1}-r_{2}-r_{3}-\sum_{n=2}^{N-1} r_{n+2}-2 r_{N+2}-\sum_{n=2}^{N-1} r_{N+n+1}=0 \\
k_{1}\left(a_{C O} a_{H_{2}}^{2}-\frac{a_{C H_{2}} a_{H_{2} O}}{K_{a, 1}}\right)-k_{2}\left(a_{C H_{2}} a_{H_{2}}-\frac{a_{C H_{4}}}{K_{a, 2}}\right)-k_{3}\left(a_{C H_{2}} a_{C H_{4}}-\frac{a_{C_{2} H_{6}}}{K_{a, 3}}\right) \\
-\sum_{n=2}^{N-1} k_{4}\left(a_{C H_{2}} a_{C_{n} H_{2 n+2}}-\frac{a_{C_{n+1} H_{2(n+1)+2}}}{K_{a, n+2}}\right)-2 k_{5}\left(a_{C H_{2}}^{2}-\frac{a_{C_{2} H_{4}}}{K_{a, N+2}}\right) \\
-\sum_{n=2}^{N-1} k_{5}\left(a_{C H_{2}} a_{C_{n} H_{2 n}}-\frac{a_{C_{n+1} H_{2(n+1)}}}{K_{a, N+n+1}}\right)=0
\end{array}
$$

Equation 4.7 can easily be solved using the quadratic formula. In this way, a closed form solution for the activity of $\mathrm{CH}_{2}$, Equation 4.8, can be obtained using the pseudo-steady state approximation.

$$
a_{C H_{2}}=\frac{-B+\sqrt{B^{2}-4 A C}}{2 A}
$$

\footnotetext{
${ }^{2}$ The same result occurs when the net rate for $\mathrm{CH}_{2}$ is written by considering the stoichiometry of reaction step 1-5. This expression is then equated to zero.
} 
where

$$
\begin{gathered}
A=2 k_{5} \\
B=\frac{k_{1}}{K_{a, 1}} a_{H_{2} O}+k_{2} a_{H_{2}}+k_{3} a_{C} H_{4}+k_{4} \sum_{n=2}^{N-1} a_{C_{n} H_{2 n+2}}+k_{5} \sum_{n=2}^{N-1} a_{C_{n} H_{2 n}} \\
C=-k_{1} a_{C O} a_{H_{2}}^{2}-\frac{k_{2}}{K_{a, 2}} a_{C H_{4}}-\frac{k_{3}}{K_{a, 3}} a_{C_{2} H_{6}}-\sum_{n=2}^{N-1} \frac{k_{4}}{K_{a, n+2}} a_{C_{n+1} H_{2(n+1)+2}}-2 \frac{k_{5}}{K_{a, N+2}} a_{C_{2} H_{4}} \\
-\sum_{n=2}^{N-1} \frac{k_{5}}{K_{a, N+n+1}} a_{C_{n+1} H_{2(n+1)}}
\end{gathered}
$$

Since the activity of any species $i$ is an intensive, positive property, the values of $A$ and $B$ are always positive while $C$ is always negative. The value of the discriminant, $B^{2}-4 A C$, will always be $>0$. The roots of Equation 4.7 are therefore always real, unequal and opposite in sign. Only the positive root given by Equation 4.8 is physically meaningful and is solved for. The activity of $\mathrm{CH}_{2}$ is then explicitly dependent on the activity of $\mathrm{CO}, \mathrm{H}_{2}$ and the n-paraffin and 1-olefin products and can be eliminated from the rate expressions in Table 4.2 .

\subsection{Thermodynamic Consistency}

It is important to assess the behaviour and thermodynamic consistency of the kinetic model at complete chemical equilibrium. This analysis involves setting the rate expression for each reaction in Table 4.2 to zero. To begin, consider reaction step 1 and 5 . The equilibrium constant for reaction step 1 is given by Equation 4.9 and for $n=1$ in reaction step 5, the equilibrium constant is given by Equation 4.10 .

$$
\begin{gathered}
K_{a, 1}=\frac{a_{C H_{2}} a_{H_{2} O}}{a_{C O} a_{H_{2}}^{2}} \\
K_{a, N+2}=\frac{a_{C_{2} H_{4}}}{a_{C H_{2}}^{2}}
\end{gathered}
$$

The product of $K_{a, 1}$ and $K_{a, N+2}^{\frac{1}{2}}$ allows the activity of $\mathrm{CH}_{2}$ to be eliminated in Equation 4.11. This yields the equilibrium constant for the formation of ethene from the hydrogenation of $\mathrm{CO}$, on a mole CO basis.

$$
K_{a, 1} K_{a, N+2}^{\frac{1}{2}}=\frac{a_{C_{2} H_{4}}^{\frac{1}{2}} a_{H_{2} \mathrm{O}}}{a_{\mathrm{CO}} a_{H_{2}}^{2}}
$$


By following a similar procedure, the equilibrium constant for the formation of propene from the hydrogenation of $\mathrm{CO}$, also on a mole $\mathrm{CO}$ basis, is given by Equation 4.12

$$
K_{a, 1}\left(K_{a, N+2} K_{a, N+3}\right)^{\frac{1}{3}}=\frac{a_{C_{3} H_{6}}^{\frac{1}{3}} a_{H_{2} O}}{a_{C O} a_{H_{2}}^{2}}
$$

In general, the equilibrium constant for the formation of any 1-olefin from the hydrogenation of $\mathrm{CO}$, on a mole $\mathrm{CO}$ basis, is given by Equation 4.13 .

$$
K_{a, 1}\left(\prod_{i=1}^{n} K_{a, N+i+1}\right)^{\frac{1}{n}}=\frac{a_{C_{n} H_{2 n}}^{\frac{1}{n}} a_{H_{2} O}}{a_{C O} a_{H_{2}}^{2}}
$$

It is easy to see that the product of $K_{a, 1}$ and $K_{a, 2}$ yields the equilibrium constant for methane formation directly from the hydrogenation of $\mathrm{CO}$ on a mole $\mathrm{CO}$ basis. This is shown in Equation 4.14.

$$
K_{a, 1} K_{a, 2}=\frac{a_{C H_{4}} a_{H_{2} O}}{a_{C O} a_{H_{2}}^{3}}
$$

An interesting result arises when reaction steps 2, 3 and 4 are considered. The product of the equilibrium constants $K_{a, 2}$ and $K_{a, 3}$ gives Equation 4.15. By substituting in the equilibrium constant $K_{a, N+2}$, the equilibrium constant for the hydrogenation of ethene results in Equation 4.16 .

$$
\begin{aligned}
& K_{a, 2} K_{a, 3}=\frac{a_{C_{2} H_{6}}}{a_{C H_{2}}^{2} a_{H_{2}}} \\
& \frac{K_{a, 2} K_{a, 3}}{K_{a, N+2}}=\frac{a_{C_{2} H_{6}}}{a_{C_{2} H_{4}} a_{H_{2}}}
\end{aligned}
$$

In general, by following a similar procedure, the hydrogenation equilibrium constant of any 1-olefin to the corresponding n-paraffin of the same carbon number is given by Equation 4.17. The net independent reactions that result from this analysis are summarised in Table 4.3. The kinetic model developed is thus thermodynamically consistent and satisfies Criterion 3.

$$
\frac{K_{a, 2} \prod_{i=1}^{n} K_{a, i+2}}{\prod_{i=1}^{n} K_{a, N+i+1}}=\frac{a_{C_{n} H_{2 n+2}}}{a_{C_{n} H_{2 n}} a_{H_{2}}}
$$

This analysis demonstrates that the FT reaction system consists of a number of subsystems working to achieve equilibrium (Norval, 2008). The large equilibrium constant of the methanation reaction in Table 4.3 results in methane being the dominant product at complete chemical equilibrium (Subiranas, 2009). The large thermodynamic driving force to form methane at higher CO conver- 
Table 4.3: Net reactions at complete chemical equilibrium

\begin{tabular}{lc}
\hline Reaction name & Net chemical reaction \\
\hline 1. Methanation & $\mathrm{CO}+3 \mathrm{H}_{2} \rightleftharpoons \mathrm{CH}_{4}+\mathrm{H}_{2} \mathrm{O}$ \\
2. 1-Olefin formation $(n \geq 2)$ & $\mathrm{CO}+2 \mathrm{H}_{2} \rightleftharpoons \frac{1}{n} \mathrm{C}_{n} \mathrm{H}_{2 n}+\mathrm{H}_{2} \mathrm{O}$ \\
3. 1-Olefin hydrogenation $(n \geq 2)$ & $\mathrm{C}_{n} \mathrm{H}_{2 n}+\mathrm{H}_{2} \rightleftharpoons \mathrm{C}_{n} \mathrm{H}_{2 n+2}$ \\
4. Water gas shift (WGS) & $\mathrm{CO}+\mathrm{H}_{2} \mathrm{O} \rightleftharpoons \mathrm{H}_{2}+\mathrm{CO}_{2}$ \\
\hline
\end{tabular}

sions, as the reaction system approaches complete chemical equilibrium, is a possible explanation for the increase in methane selectivity in the study of Tucker \& van Steen (2019). The interplay between reaction steps 2 and 3 in Table 4.3 accounts for the large yield of direct hydrogenation of 1-olefins observed in the study of Schulz \& Claeys (1999). It also reiterates the importance of secondary 1-olefin hydrogenation. Net reaction 2 in Table 4.3 is also in agreement with the large primary molar content of 1-olefins in hydrocarbon fractions (Claeys \& van Steen, 2004). Finally, net reaction 4 in Table 4.3 results from the favourable equilibrium position of the WGS reaction at the conditions of LTFT synthesis. From Table 4.3, 1-olefin isomerisation to cis/trans 2-olefins has been neglected. The isomerisation of 1-olefins to 2-olefins via double bond shift becomes more important at higher $\mathrm{CO}$ conversions, suggesting competitive adsorption between $\mathrm{CO}$ and 1-olefins (Bukur et al., 2012; Tucker \& van Steen, 2019). However, while thermodynamically favourable, 1-olefin isomerisation leads to a product that has no effect on the product distribution (Claeys \& van Steen, 2004). Net reaction 3 in Table 4.3 is also indicative of complete hydrogenation, favouring a predominantly saturated product. The isomerisation of 1-olefins can thus be neglected because of the eventual hydrogenation of cis/trans 1-olefin isomers to the corresponding n-paraffin of the same carbon number (Horiuti \& Polanyi, 1934).

\subsection{Model Demonstration}

The purpose of this section is to demonstrate the effectiveness of the proposed reaction pathway and kinetic model in simulating FT reaction behaviour. To illustrate this, a fixed-bed reactor (FBR) will be used to perform all calculations. The design equation with respect to the weight of catalyst is given by Equation 4.18, assuming ideal plug flow. In Equation $4.18, k_{j}$ refers to the rate constants in reaction steps $1-6$ in $\mathrm{mol} / \mathrm{kg} / \mathrm{s}$ and $a_{i}$ refers to the activity of species $i$.

$$
\frac{d F_{i}}{d W}=r_{i}\left(k_{j}, a_{i}\right)
$$


Equation 4.18 can be normalised with respect to the total weight of catalyst to give Equation 4.19. This enables Equation 4.19 to always be integrated between the bounds zero and one.

$$
\frac{d F_{i}}{d x}=r_{i}\left(k_{j}, a_{i}\right) W_{\text {total }} ; \quad W=x W_{\text {total }}
$$

In addition to ideal plug flow conditions, the following assumptions are made:

1. The reactor is isothermal and isobaric.

2. All chemical species remain in the vapour phase.

Both assumptions allow the behaviour of the reaction kinetics to be studied. This prevents any observations in the kinetic model behaviour from being disguised by or attributed to temperature rises, pressure-drop and particularly in the case of Assumption 2, liquid formation. The values of the rate constants $k_{1}-k_{6}$ used in this demonstration are summarised in Table 4.4 and are chosen such that typical FT reaction behaviour (high methane yield, low ethene yield, and the change from mainly 1-olefins at low carbon numbers to mainly n-paraffins at high carbon numbers) is observed. Furthermore, the choice of rate constants in Table 4.4 enables $\mathrm{CO}$ hydrogenation to be ratedetermining and ensures that the rate of chain growth is rapid in comparison. This is consistent with the equilibrium control in the product distribution (Norval, 2008). The highest carbon number $n$-paraffin and 1-olefin present in the simulation is $C_{81}(N=81)$, to ensure an accurate simulation of the product distribution up to $\mathrm{C}_{80}$. This choice is validated in Appendix A.2.

Table 4.4: Numerical values of the rate constants in reaction step 1-6

\begin{tabular}{cc}
\hline Rate constant & Value $[\mathrm{mol} / \mathrm{kg} / \mathrm{s}]$ \\
\hline$k_{1}$ & 0.0001 \\
$k_{2}$ & 0.001 \\
$k_{3}$ & 0.1 \\
$k_{4}$ & 10 \\
$k_{5}$ & 1 \\
$k_{6}$ & 0.0001 \\
\hline
\end{tabular}

The behaviour of the $\mathrm{FT}$ reaction system is simulated at a CO conversion of $10 \%$, total pressure of $20 \mathrm{bar}(\mathrm{a})$, temperature of $220^{\circ} \mathrm{C}$ and $\mathrm{H}_{2} / \mathrm{CO}$ feed ratio of 2.1 (Visconti et al., 2007, 2011). Low conversion runs are typically used to develop kinetic models and are used by literature kinetic models as evidence of the adequate prediction of the observations in the FT product distribution. In this way, the strengths of the equilibrium approach used in the model developed here is most evident i.e. how chemical equilibrium controls the product distribution.

In Figure 4.2a, the prediction of the production distribution of the n-paraffin and 1-olefin homol- 
ogous series as a function of carbon number is shown. The model can effectively account for the high methane yield, low ethene yield, and the change from mainly 1-olefins at low carbon numbers to mainly n-paraffins at high carbon numbers.

The hydrogenation of $\mathrm{CH}_{2}$ leading to the formation of methane is the most thermodynamically favourable reaction step because of the large value of $K_{a, 2} 2^{3}$. Since $k_{3}$ is two orders of magnitude smaller than $k_{4}$, methane is also consumed at a slower rate in comparison to other n-paraffins of higher carbon number leading to the high methane yield. In the kinetic model formulation, ethene forms from $\mathrm{CH}_{2}$ coupling, which is thermodynamically unfavourable (Claeys \& van Steen, 2004; Mousavi et al., 2015). This is supported by the small value of $K_{a, N+2}$. Ethene is also the first 1-olefin that forms. The series nature of the 1-olefin chain growth reactions means that ethene reacts to form 1-olefins of higher carbon number. The low thermodynamic driving force to form ethene together with its consumption to form higher 1-olefins leads to the low ethene yield.

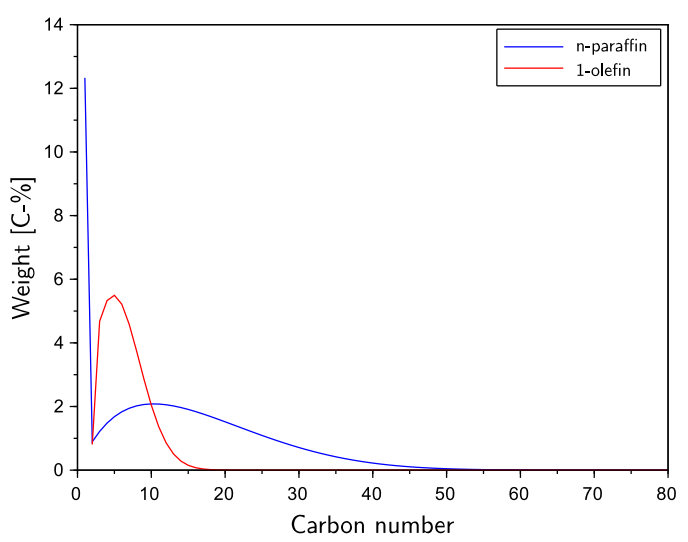

(a) Product distribution

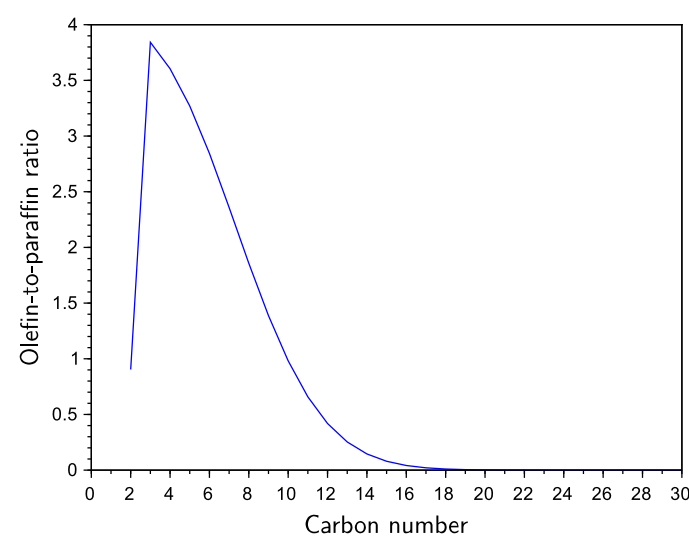

(b) Olefin-to-paraffin ratio

Figure 4.2: N-paraffin and 1-olefin product distribution and olefin-to-paraffin ratio as a function of carbon number at a $\mathrm{CO}$ conversion of $10 \%, 220^{\circ} \mathrm{C}, 20 \mathrm{bar}(\mathrm{a})$ and $\mathrm{H}_{2} / \mathrm{CO}$ feed ratio of 2.1

The small value of $K_{a, N+2}$ favours a distribution amongst the low carbon number 1-olefins. The equilibrium constant $K_{a, N+3}$ for the formation of propene in the kinetic model is four orders of magnitude larger than that of $K_{a, N+2}$. Thereafter the equilibrium constants $K_{a, N+4}-K_{a, 2 N}$ are all equal to unity, due to the linear variation of the ideal gas Gibbs free energy of formation with carbon number in the 1-olefin homologous series. The driving force to form propene from ethene is therefore greatest. An equal thermodynamic driving force thereafter exists to form 1-butene from propene, 1-pentene from 1-butene and so on, leading to the observed decrease in 1-olefin content.

\footnotetext{
${ }^{3}$ Further detail on the numerical values of the equilibrium constants as a function of temperature is given in Appendix A.3
} 
The addition of $\mathrm{H}_{2}$, however, favours the incorporation of $\mathrm{CH}_{2}$ into long chain n-paraffins to a greater extent (Mousavi et al., 2015). This is supported by the equilibrium constant $K_{a, 3}$ being two orders of magnitude larger than $K_{a, N+2}$. The equilibrium constants $K_{a, 4} K_{a, N+1}$ are thereafter all equal to unity, due to the linear variation of the Gibbs free energy of formation with carbon number in the $n$-paraffin homologous series. An equal thermodynamic driving force thereafter exists to form n-paraffins of carbon number $C_{3}$ and greater. The equilibrium constants $K_{a, 4}-K_{a, N+1}$ are however an order of magnitude larger than $K_{a, 3}$. This together with the higher reactivity due to the magnitude of $k_{4}$ relative to $k_{3}$ and series nature of the $n$-paraffin chain growth reaction favours a distribution amongst the high carbon number n-paraffins. The decrease in olefin-to-paraffin ratio in Figure $4.2 \mathrm{~b}$ from carbon number $\mathrm{C}_{3}$ onwards therefore arises because of the shift in the product distribution from 1-olefins of low carbon number to n-paraffins of high carbon number.

In Figure 4.3a, a semi-logarithmic plot of the total product distribution as a function of carbon number is shown. The global maximum observed at $C_{1}$ is caused by the large thermodynamic driving force to form methane through the hydrogenation of $\mathrm{CH}_{2}$. The low $\mathrm{C}_{2}$ selectivity arises because of the combination of the thermodynamically unfavourable $\mathrm{CH}_{2}$ coupling to ethene and the rapid reaction of ethane towards higher carbon number n-paraffins. The 1-olefin content is greatest between carbon numbers $C_{3}-C_{10}$. The local maximum observed at $C_{3}$ thus arises because propene is the most thermodynamically favoured 1-olefin. A change in concavity in Figure 4.3a arises at about $C_{10}$ because of the changeover from mostly 1-olefins at low carbon number to mostly n-paraffins of carbon number $\mathrm{C}_{10}$ and greater.

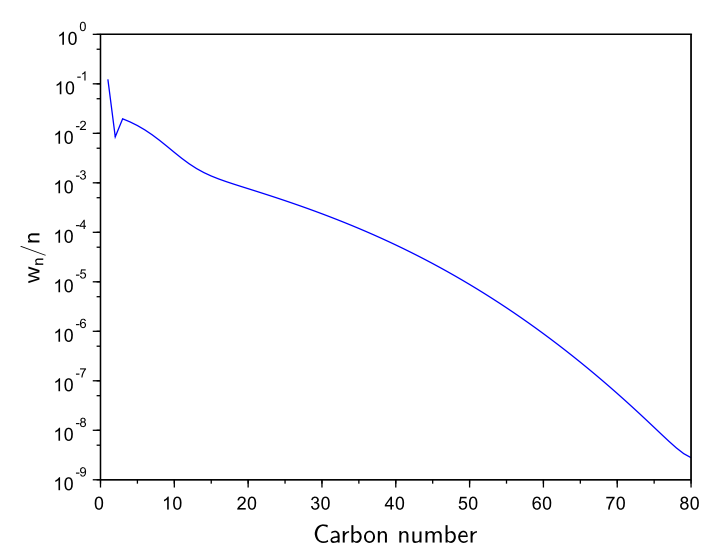

(a) ASF type distribution

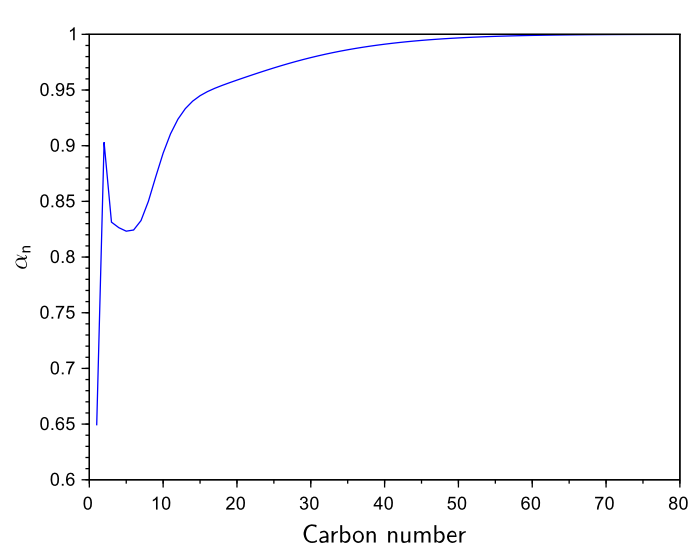

(b) $\alpha$ as a function of carbon number

Figure 4.3: Semi-logarithmic plot of the hydrocarbon distribution and chain growth probability, both as functions of carbon number at a $\mathrm{CO}$ conversion of $10 \%, 220^{\circ} \mathrm{C}, 20$ bar(a) and $\mathrm{H}_{2} / \mathrm{CO}$ feed ratio of 2.1 
This behaviour gives rise to carbon number specific chain growth probabilities in Figure $4.3 \mathrm{~b} f$. The value of $\alpha$ calculated corresponds to the trends observed in Figure 4.3a. The definition of $\alpha$ is the probability of chain growth. Low $\alpha$ at a specific carbon number thus indicates a low probability of chain growth and vice versa. This is clear from the low $\alpha$ at $C_{1}$, which is reflected by its lower reactivity for chain growth relative to other $n$-paraffins. In comparison, the increase in $\alpha$ and peak at $C_{2}$ indicates its high reactivity for chain growth since it acts as chain starter in the case of ethene. From $C_{10}$ onwards, the value of $\alpha$ increases, favouring chain growth towards higher carbon numbers, specifically n-paraffins. The value of $\alpha \rightarrow 1$ as the carbon number increases up to $\mathrm{C}_{80}$. This indicates that $N=81$ allows for an accurate simulation and description of the product distribution up to $\mathrm{C}_{80}$ (see Appendix A.2).

By considering the partial equilibrium aspects involved in the chain growth reactions of the FT reaction system, the observed occurrences in the FT product distribution can effectively be accounted for in a light weight and easily tractable manner. The element balance closure of this simulation is summarised in Table 4.5. All balances are satisfied within the limit of machine precision. The outlined approach is therefore both physically meaningful, thermodynamically consistent and represents a good foundation to carry out further testing.

Table 4.5: Element balance closure from model demonstration

\begin{tabular}{ccccc}
\hline Element & In $[\mathrm{mol} / \mathrm{s}]$ & Out $[\mathrm{mol} / \mathrm{s}]$ & Absolute error $[\mathrm{mol} / \mathrm{s}] \times 10^{14}$ & Relative error $[-] \times 10^{14}$ \\
\hline $\mathrm{C}$ & 1 & 1 & 0.0444 & 0.0444 \\
$\mathrm{H}$ & 4.2 & 4.2 & -0.711 & -0.169 \\
$\mathrm{O}$ & 1 & 1 & 0.0111 & 0.0111 \\
\hline
\end{tabular}

\subsection{Effect of Operating Conditions}

It is important to study the effect of $\mathrm{CO}$ conversion, temperature, pressure and $\mathrm{H}_{2} / \mathrm{CO}$ feed ratio on the FT product distribution and selectivity. This provides a fair test of the model to ensure that it produces the correct response over a wide range of operating conditions. The base case conditions correspond to a CO conversion of $40 \%$, temperature of $220^{\circ} \mathrm{C}$, total pressure of 20 bar(a) and $\mathrm{H}_{2} / \mathrm{CO}$ ratio of 2.1. Again, the highest carbon number n-paraffin and 1-olefin present in the simulation is $\mathrm{C}_{81}(N=81)$. The model response to changes in operating conditions will be examined using plots of the total hydrocarbon, n-paraffin and 1-olefin product distribution as a function of carbon number and the selectivity, on a carbon basis, to $C_{1}, C_{2}, C_{3}, C_{4}, C_{5+}$ and $C_{2}$ as a function of the condition investigated.

\footnotetext{
${ }^{4}$ The chain growth probabilities in Figure $4.3 b$ are calculated by solving $w_{n}=n\left(1-\alpha^{2}\right) \alpha^{n-1}$ for $\alpha$.
} 
The selectivity is lumped based on carbon number and calculated on a carbon basis using Equation 4.20, where $F_{i}$ is the molar flow rate of species $i, n_{i}$ is the number of carbon atoms in species $i$, $F_{C O, 0}$ is the feed flow rate of $\mathrm{CO}$ and $X_{C O}$ is the CO conversion.

$$
S_{i}=\frac{F_{i} n_{i}}{F_{C O, 0} X_{C O}} \times 100
$$

For clarity, $C_{1}$ refers to methane, $C_{2}$ refers to ethane and ethene, $C_{3}$ refers to propane and propene, $\mathrm{C}_{4}$ refers to $\mathrm{n}$-butane and 1 -butene and $\mathrm{C}_{5+}$ refers to all $\mathrm{n}$-paraffins and 1-olefins of carbon number $\mathrm{C}_{5}$ and greater. The selectivity to $\mathrm{C}_{1}, \mathrm{C}_{2}, \mathrm{C}_{3}, \mathrm{C}_{4}$ and $\mathrm{CO}_{2}$ are calculated using Equation 4.20 . The selectivity to $C_{5+}$ can then be calculated using Equation 4.21 (Rafiq et al., 2011; Bukur et al., 2012).

$$
S_{C_{5+}}=100-\left(S_{C_{1}}+S_{C_{2}}+S_{C_{3}}+S_{C_{4}}+S_{C O_{2}}\right)
$$

\subsubsection{Effect of CO Conversion}

The effect of $\mathrm{CO}$ conversion on the product distribution and carbon-based selectivity is simulated by keeping the feed temperature, total pressure and $\mathrm{H}_{2} / \mathrm{CO}$ feed ratio fixed at $220^{\circ} \mathrm{C}, 20 \operatorname{bar}(\mathrm{a})$ and 2.1 respectively. The total hydrocarbon, n-paraffin and 1-olefin product distribution product distributions are then compared at CO conversions of 20\%,40\%,60\%,80\% and 95\% in Figure $4.4 \mathrm{a}, 4.4 \mathrm{~b}$ and $4.4 \mathrm{C}$. The carbon-based selectivity curves as a function of conversion are studied up to a CO conversion of $95 \%$ in Figure $4.4 \mathrm{~d}$.

In Figure 4.4a, increasing the CO conversion from $20 \%$ to $60 \%$ results in a shift in the product distribution from low carbon numbers to high carbon numbers. This is shown by the depression in the curves in the carbon number range between $C_{2}-C_{10}$ leading to an observed rise in the curves in the carbon number range between $C_{10}-C_{25}$ for $C O$ conversions between $20 \%$ to $60 \%$. This is consistent with the findings of Iglesia et al. (1991) in which an increase in CO conversion increases the average molecular weight of the product. In Figure 4.4b, a rise in the n-paraffin curves is observed between $\mathrm{C}_{2}-\mathrm{C}_{20}$ for $\mathrm{CO}$ conversions between 20-60\%. In contrast, a depression is observed in the 1-olefins curves in Figure $4.4 \mathrm{C}$ between $\mathrm{C}_{2}-\mathrm{C}_{12}$, after which the curves rise for carbon numbers greater than $C_{12}$. Further increases in $C O$ conversion from $60 \%$ to $95 \%$ results in the reverse behaviour in Figure 4.4 a with a shift to low carbon number products. This is shown by the rise in methane and $C_{2}-C_{10}$ weight fractions in Figure $4.4 \mathrm{a}$ and Figure $4.4 \mathrm{~b}$. In Figure $4.4 \mathrm{~d}$, the selectivity to the lumped product groups remains almost unchanged, apart from a steady rise in $\mathrm{CO}_{2}$ selectivity up to a $\mathrm{CO}$ conversion of $60 \%$. However, the $\mathrm{C}_{1}$ and $\mathrm{CO}_{2}$ selectivity increases more rapidly with increases in CO conversion from $60 \%$ to $95 \%$ corresponding with a rapid decline in $C_{5+}$ selectivity. This is consistent with the findings of Tucker \& van Steen (2019). 
The results presented in Figure 4.4a and 4.4d reiterates that the selectivity to long chain hydrocarbons is kinetically controlled in FT synthesis. Increases in CO conversion increases the formation of the monomer $\mathrm{CH}_{2}$ since reaction step 1 is essentially irreversible. The preferential hydrogenation of $\mathrm{CH}_{2}$ to methane and its subsequent consumption in the $\mathrm{n}$-paraffin chain growth reactions leads to the observed rise in the n-paraffin product distribution for carbon numbers between $C_{2}-C_{20}$ in Figure $4.4 \mathrm{~b}$ up to a CO conversion of $60 \%$. The observations mentioned above arise because of the series nature of the n-paraffin and 1-olefin chain growth reactions. In Figure 4.4C, the depression in the 1-olefin curves for carbon numbers $C_{2}-C_{12}$ and rise in the curves from $C_{12}$ onwards for $C O$ conversions between $20-60 \%$ is the result of the consumption of 1-olefins of low carbon number to form 1-olefins of high carbon number. This behaviour of the 1-olefins continues until a $\mathrm{CO}$ conversion of $80 \%$.

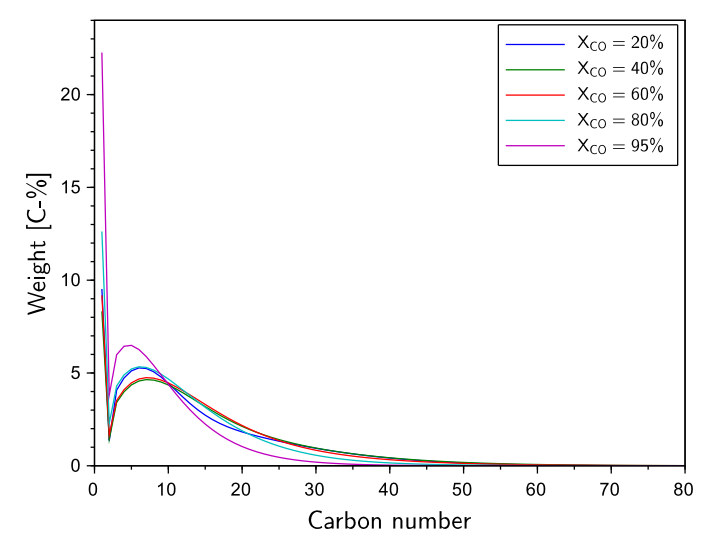

(a) Total hydrocarbon product distribution

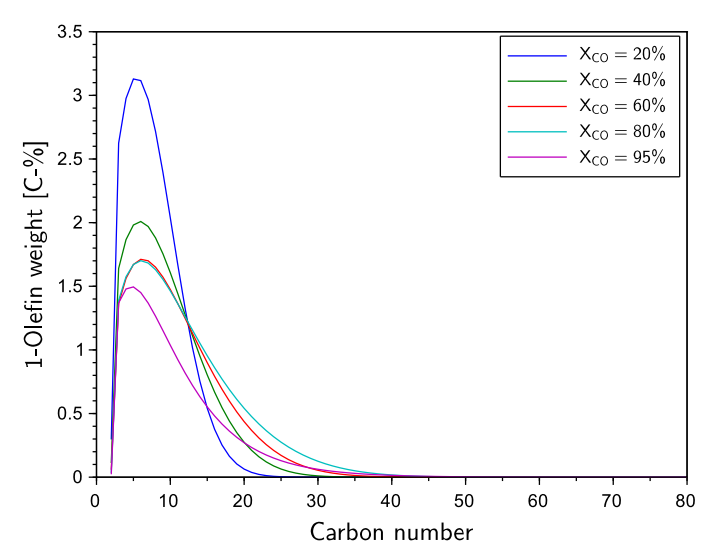

(c) 1-Olefin product distribution

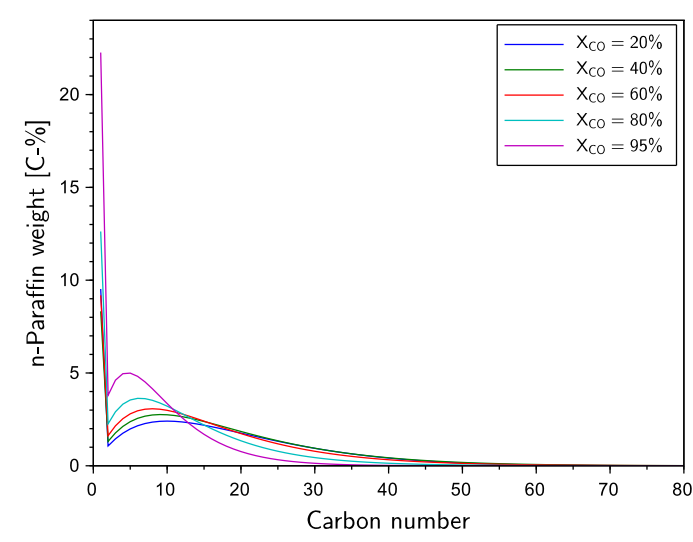

(b) n-Paraffin product distribution

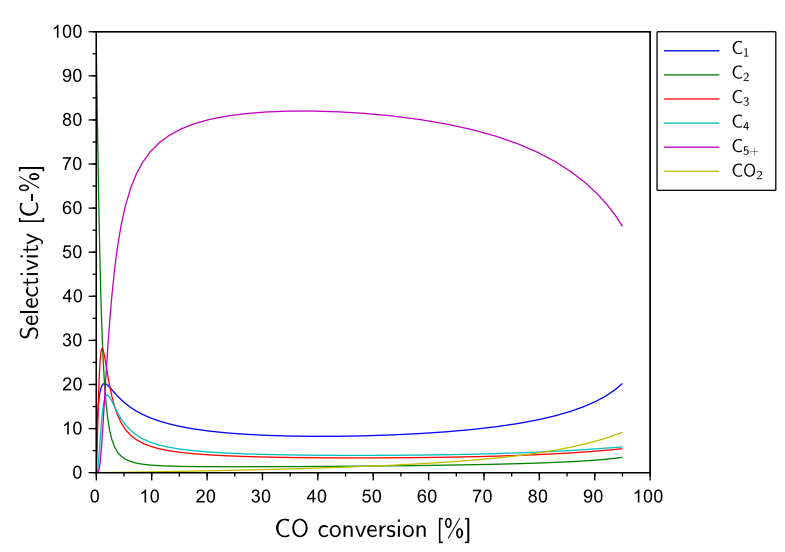

(d) Selectivity as a function of CO conversion

Figure 4.4: The effect of $\mathrm{CO}$ conversion on the total hydrocarbon, n-paraffin and 1-olefin product distributions and carbon-based selectivity at $220^{\circ} \mathrm{C}, 20 \mathrm{bar}(\mathrm{a})$ and an $\mathrm{H}_{2} / \mathrm{CO}$ feed ratio of 2.1 
In Figure 4.4d, from a CO conversion of $60 \%$, an increase in methane selectivity is observed. This is a consequence of the reverse $n$-paraffin growth reaction being favoured. As such, a chemical feedback occurs in which the rate of the reverse n-paraffin chain growth reaction increases. This leads to a shift from high carbon number n-paraffins to low carbon number n-paraffins. The rate of methane consumption in the $n$-paraffin chain growth reaction reduces, while the increased formation of $\mathrm{CH}_{2}$ results in its preferential hydrogenation towards methane. The rapid consumption of $\mathrm{CH}_{2}$ limits the formation of 1-olefins as the system tends to a thermodynamically favoured saturated product. This is observed by the gradual decrease in 1-olefin content across all carbon numbers in Figure 4.4c. The increase in methane selectivity in Figure $4.4 \mathrm{~d}$ is also a consequence of the increased $\mathrm{CO}_{2}$ selectivity (Tucker \& van Steen, 2019). The formation of water in reaction step 1 drives the forward WGS reaction. This favours the formation of $\mathrm{H}_{2}$ and the preferential hydrogenation of $\mathrm{CH}_{2}$ to methane. This is observed in Figure $4.4 \mathrm{~b}$ as the rise in the methane weight fraction and $\mathrm{C}_{1}$ selectivity, corresponding to a decrease in $\mathrm{C}_{5+}$ selectivity in Figure $4.4 \mathrm{~d}$ from $\mathrm{CO}$ conversions of $60-95 \%$. Evidently, a large thermodynamic driving force exists to form methane as complete CO conversion is approached, which is expected (Subiranas, 2009).

\subsubsection{Effect of Temperature}

The effect of temperature on the product distribution and carbon-based selectivity is simulated by keeping the $\mathrm{CO}$ conversion, total pressure and $\mathrm{H}_{2} / \mathrm{CO}$ feed ratio fixed at 40\%, 20 bar(a) and 2.1 respectively. The values of the rate constants used are given in Table 4.4. As such, the observed changes in the product distribution and selectivity arise due to changes in the equilibrium constants with temperature (see Appendix A.3). The total hydrocarbon, n-paraffin and 1-olefin product distribution product distribution are then compared at $180^{\circ} \mathrm{C}, 200^{\circ} \mathrm{C}, 220^{\circ} \mathrm{C}, 240^{\circ} \mathrm{C}$ and $260^{\circ} \mathrm{C}$ in Figure 4.5a, 4.5b and 4.5c, the operating temperature range of LTFT (Méndez et al., 2017). The carbon-based selectivity curves as a function of temperature are studied up to a temperature of $260^{\circ} \mathrm{C}$ in Figure $4.5 \mathrm{~d}$.

With increases in temperature in Figure 4.5a, there is an increased shift from high carbon number to low carbon number products, shown by the rise in the curves for carbon numbers between $\mathrm{C}_{2}$ $\mathrm{C}_{10}$. In Figure 4.5b, there is a gradual decrease in n-paraffin content, with a shift in the n-paraffin distribution from high to low carbon number products. In Figure $4.5 \mathrm{C}$, there is a consistent rise in 1-olefin content particularly for carbon numbers between $C_{2}-C_{10}$. As a result of the shift towards low carbon number products, there is an increase in the selectivity to $C_{3}$ and $C_{4}$ corresponding to a decrease in $\mathrm{C}_{5+}$ selectivity with increasing temperature in Figure $4.5 \mathrm{~d}$.

The observed shift towards low carbon number products with increasing temperature is consistent with the expected thermodynamic behaviour in an exothermic reaction (Claeys \& van Steen, 2004). 
The decrease in the n-paraffin content and shift to low carbon numbers can be attributed to the increased thermodynamic driving force to form 1-olefins with increasing temperature. This is consistent with net reaction 3 in Table 4.3, where dehydrogenation becomes more favourable with increasing temperature. $\mathrm{CH}_{2}$ coupling, which leads to 1-olefin formation, is then favoured. The preferential consumption of $\mathrm{CH}_{2}$ in the 1-olefin chain growth reactions thus limits the amount of $\mathrm{CH}_{2}$ available for reaction in the $\mathrm{n}$-paraffin chain growth reactions leading to the observed decrease in $\mathrm{n}$-paraffin content. The reactivity of both chain growth reactions is thus driven to form products of low carbon number.

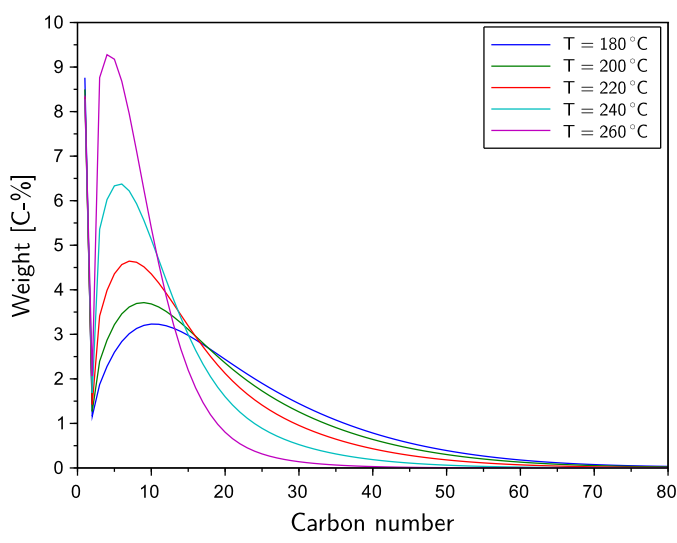

(a) Total hydrocarbon product distribution

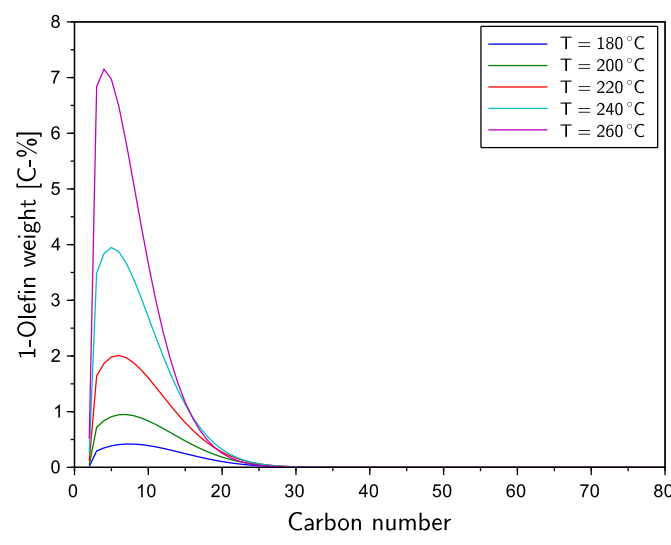

(c) 1-Olefin product distribution

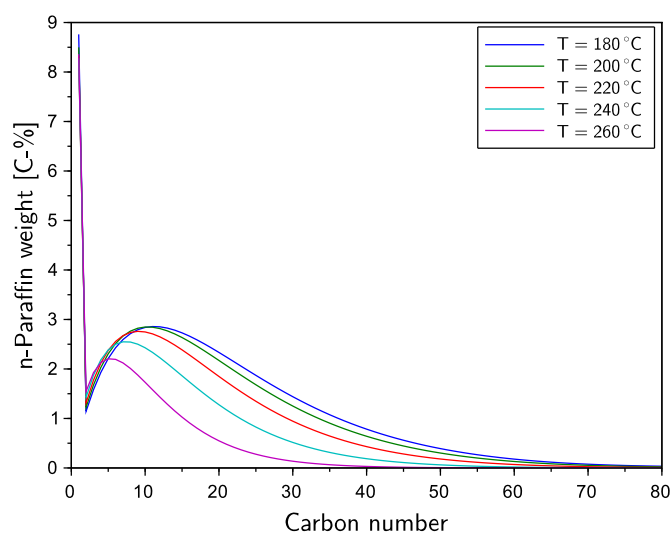

(b) n-Paraffin product distribution

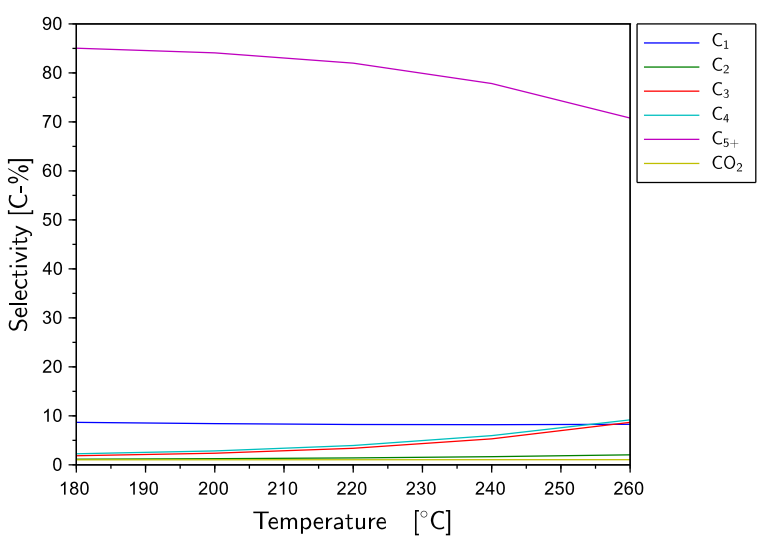

(d) Selectivity as a function of temperature

Figure 4.5: The effect of temperature on the total hydrocarbon, n-paraffin and 1-olefin product distributions and carbon-based selectivity at a CO conversion of $40 \%, 20$ bar(a) and an $\mathrm{H}_{2} / \mathrm{CO}$ feed ratio of 2.1 


\subsubsection{Effect of Pressure}

The effect of pressure on the product distribution and carbon-based selectivity is simulated by keeping the $\mathrm{CO}$ conversion, feed temperature and $\mathrm{H}_{2} / \mathrm{CO}$ feed ratio fixed at $40 \%, 220^{\circ} \mathrm{C}$ and 2.1 respectively. The total hydrocarbon, $n$-paraffin and 1 -olefin product distribution product distributions are then compared at 10, 15, 20, 25 and $30 \mathrm{bar}(\mathrm{a})$ in Figure 4.6a, 4.6b and 4.6c. The carbon-based selectivity curves are studied up to a pressure of 30 bar(a) in Figure $4.6 \mathrm{~d}$.

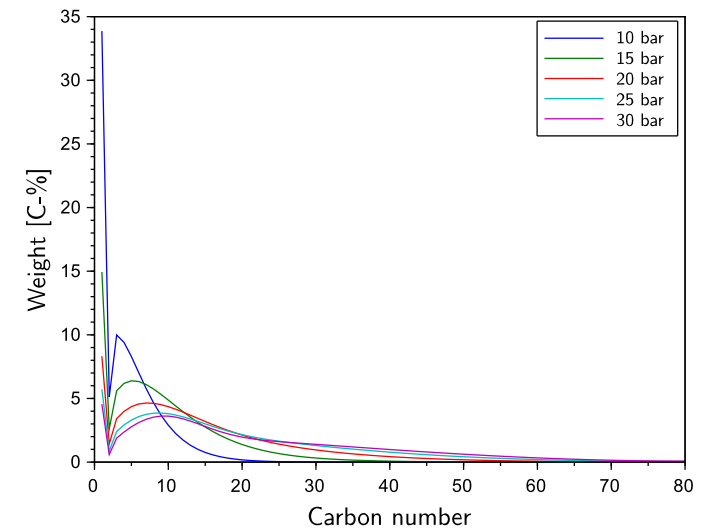

(a) Total hydrocarbon product distribution

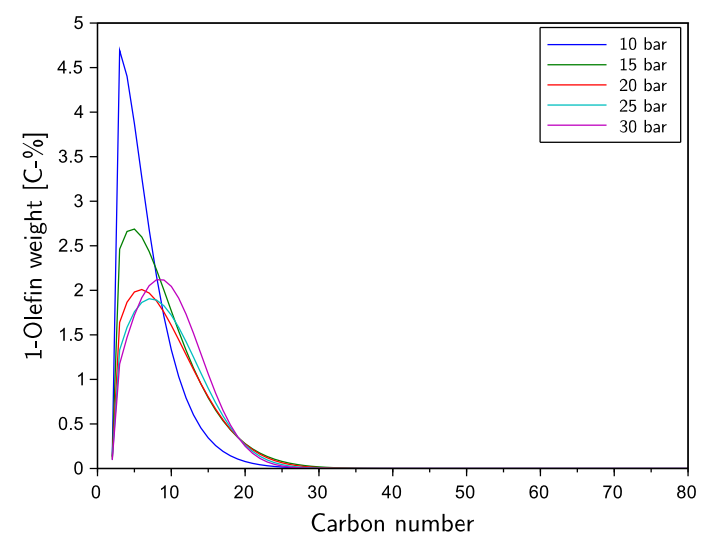

(c) 1-Olefin product distribution

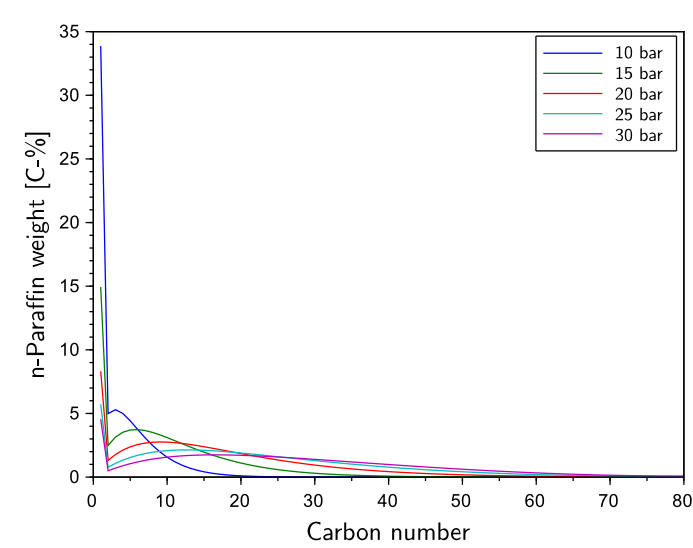

(b) n-Paraffin product distribution

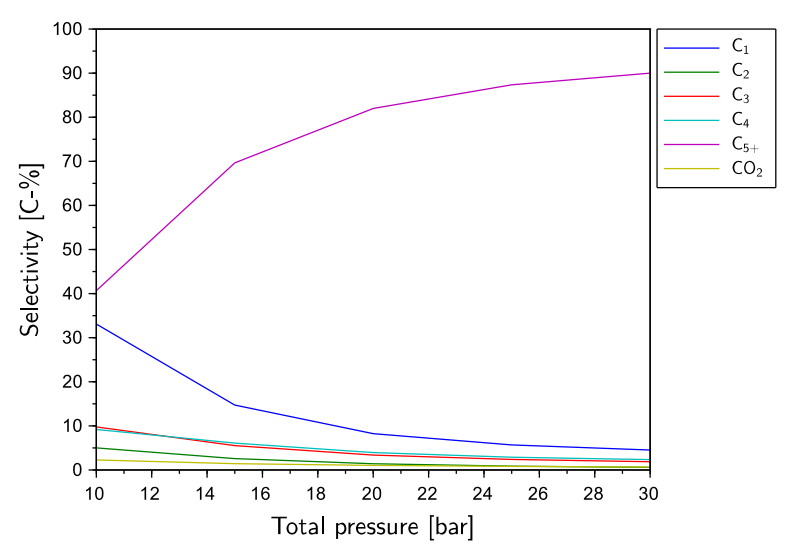

(d) Selectivity as a function of pressure

Figure 4.6: The effect of pressure on the total hydrocarbon, n-paraffin and 1-olefin product distributions and carbon-based selectivity at a CO conversion of $40 \%, 220^{\circ} \mathrm{C}$ and an $\mathrm{H}_{2} / \mathrm{CO}$ feed ratio of 2.1

In Figure 4.6a, with increasing pressure there is a decrease in methane content and a consistent shift from low to high carbon number products. Both the $n$-paraffin and 1-olefin product distributions in Figure $4.6 \mathrm{~b}$ and $4.6 \mathrm{C}$ reflect the same trend. This results in the observed increase in $\mathrm{C}_{5+}$ selectivity, corresponding with a decrease in $\mathrm{C}_{1}, \mathrm{C}_{2}, \mathrm{C}_{3}, \mathrm{C}_{4}$ and $\mathrm{CO}_{2}$ selectivity in Figure $4.6 \mathrm{~d}$. 
The observed shift from low to high carbon number products is consistent with the findings of Espinoza et al. (1999) and is expected for a vapour phase reaction. An increase in pressure thus favours the reaction which reduces the number of moles by Le Chateliers principles. CO is then preferentially hydrogenated, limiting its WGS reactivity leading to the observed decrease in $\mathrm{CO}_{2}$ selectivity with increasing pressure. In polymerisation, the forward reaction results in a reduction in the number of moles. Consequently, chain growth is favoured resulting in low carbon number products reacting to form high carbon number products. The $C_{1}, C_{2}, C_{3}$ and $C_{4}$ selectivity all decrease as a result of favourable chain growth towards $C_{5+}$ leading to the observed increase in its selectivity.

\subsubsection{Effect of $\mathrm{H}_{2} / \mathrm{CO}$ Feed Ratio}

The effect of feed ratio on the product distribution and carbon-based selectivity is simulated by keeping the CO conversion, feed temperature and total pressure fixed at $40 \%, 220^{\circ} \mathrm{C}$ and 20 $\operatorname{bar}(\mathrm{a})$. The total hydrocarbon, $\mathrm{n}$-paraffin and 1-olefin product distributions are then compared at $\mathrm{H}_{2} / \mathrm{CO}$ feed ratios of $1,1.5,2.1,2.5$ and 3 in Figure $4.7 \mathrm{a}$, 4.7b and 4.7c. The carbon-based selectivity curves as a function of conversion are studied up to an $\mathrm{H}_{2} / \mathrm{CO}$ feed ratio of 3 in Figure $4.7 \mathrm{~d}$

In Figure 4.7a, with increases in $\mathrm{H}_{2} / \mathrm{CO}$ feed ratio from 1 to 2.1 , there is initially a shift from low to high carbon numbers. Thereafter, the product distribution shifts from high carbon numbers to low carbon numbers. A similar trend is observed in the n-paraffin distribution in Figure 4.7b. In Figure 4.7c, an increase in $\mathrm{H}_{2} / \mathrm{CO}$ feed ratio reduces the 1-olefin content for all carbon numbers. In Figure 4.7d, an initial rise in $\mathrm{C}_{5+}$ selectivity is observed up to an $\mathrm{H}_{2} / \mathrm{CO}$ feed ratio of 1.5 , thereafter the $\mathrm{C}_{5+}$ selectivity decreases slowly. The $\mathrm{CO}_{2}$ selectivity decreases consistently with increasing $\mathrm{H}_{2}$ /feed ratio.

An increase in $\mathrm{H}_{2}$ content favours both $\mathrm{CO}$ and $\mathrm{CH}_{2}$ hydrogenation. This increases the forward rate of $\mathrm{CO}$ hydrogenation resulting in an increase in the formation of $\mathrm{CH}_{2}$ which is then preferentially hydrogenated to methane, favouring the n-paraffin chain growth reactions. This results in an increase in chain growth and shift from low carbon number to high carbon numbers at least up to an $\mathrm{H}_{2} / \mathrm{CO}$ feed ratio of 2.1. The preferential consumption of $\mathrm{CH}_{2}$ in the n-paraffin chain growth reactions limit 1-olefin chain growth. Further increases in the $\mathrm{H}_{2} / \mathrm{CO}$ feed ratio favours the methanation reaction, consistent with its overall reaction stoichiometry in Table 4.3. The preferential consumption of $\mathrm{CH}_{2}$ through hydrogenation limits n-paraffin chain growth leading to the shift in the product distribution to lower carbon numbers and the decrease in $\mathrm{C}_{5+}$ selectivity. This agrees with the findings of Iglesia et al. (1993). The decrease in $\mathrm{CO}_{2}$ selectivity with increasing $\mathrm{H}_{2} / \mathrm{CO}$ feed ratio is the result of the increased $\mathrm{H}_{2}$ content favouring the reverse WGS reaction 
and preferential CO hydrogenation.

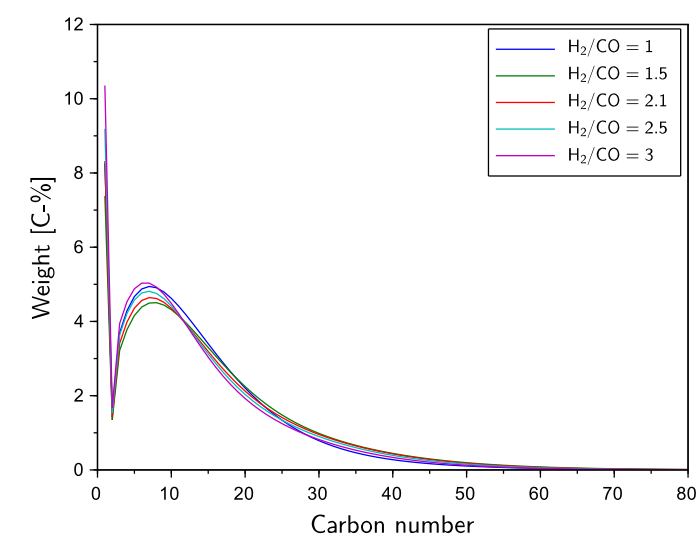

(a) Total hydrocarbon product distribution

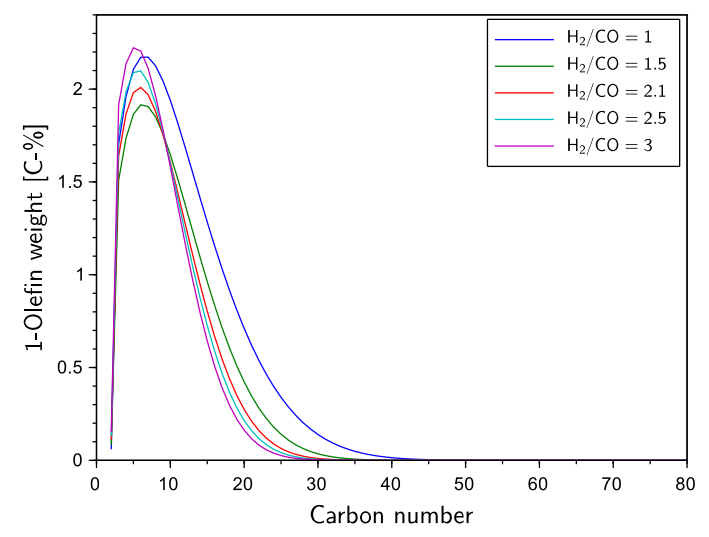

(c) 1-Olefin product distribution

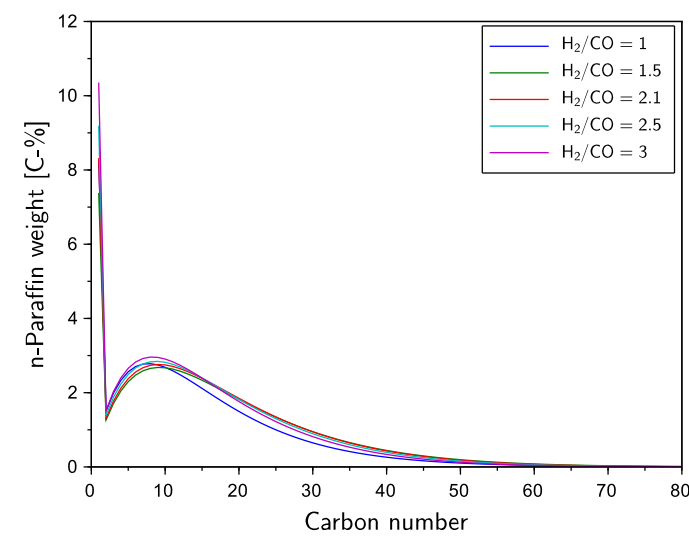

(b) n-Paraffin product distribution

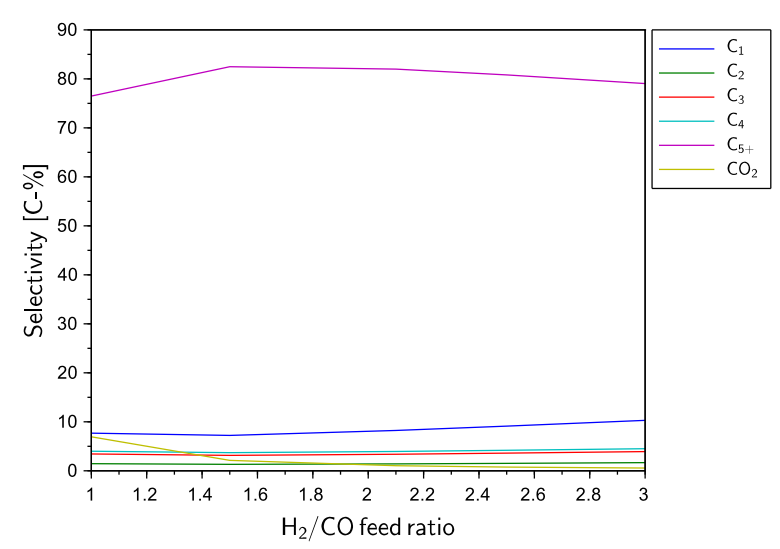

(d) Selectivity as a function of feed ratio

Figure 4.7: The effect of feed ratio on the total hydrocarbon, n-paraffin and 1-olefin product distributions and carbon-based selectivity at a CO conversion of $40 \%, 220^{\circ} \mathrm{C}$ and 20 bar(a)

\subsection{Summary}

A kinetic model has been developed for LTFT synthesis which relies on the equilibrium aspects of the reactions involved to effectively capture the known characteristics of the product distribution i.e. the high methane yield, low ethene yield and the changeover from mainly 1-olefins at low carbon numbers to mainly n-paraffins at high carbon numbers. The model demonstrates both quantitatively and qualitatively that the product distribution is equilibrium controlled while the selectivity of hydrocarbon products is kinetically controlled. This is in agreement with the work of Norval \& Phillips (1990) and Norval (2008). Furthermore, the model need not follow a Langmuir- 
Hinshelwood formulation, contrary to the trend present in literature. The assumption of partial equilibrium, through the formulation of each reaction step as an equilibrium-limited process allowed the known reaction characteristics to be captured with fewer reactions steps being required. This is since no additional assumptions regarding the carbon number dependence of the reaction rate is required while still preserving the polymerisation character of the FT reactions. The known characteristics of the FT product distribution are thus the consequence of FT chemistry (Dictor \& Bell, 1986).

The model has also been tested across a range of known FT operating conditions, assuming vapour phase conditions. A major strength is that the model is capable of reproducing the reaction behaviour over the full range of $\mathrm{CO}$ conversions, in agreement with the trends seen in Tucker \& van Steen (2019). The model also effectively predicts the changes in the product distribution with changes in operating temperature, pressure and $\mathrm{H}_{2} / \mathrm{CO}$ feed ratio. These changes have been described and can be attributed to the equilibrium basis of the model. Although this is promising, the model is limited in that the methane selectivity remained almost constant when changing operating temperature and $\mathrm{H}_{2} / \mathrm{CO}$ feed ratio. While the feed ratio is not as an important process parameter since it cannot be varied over a wide range (Claeys \& van Steen, 2004), the invariant methane selectivity with temperature can be attributed to keeping the value of the rate constants used fixed to test the equilibrium control in the FT product distribution when changing operating temperature. In both of these instances, the model is assumed to perform better when values of the rate constants are regressed from and compared to experimental data.

Overall, the equilibrium basis of the model developed here can be seen to be a success since it describes the dominant trends in the product distribution using the minimum number of input parameters. Further testing is, however, required to validate the uniqueness of the model input parameters and the model performance against experimental data. 


\section{Chapter 5}

\section{Sensitivity analysis}

During model development, it is important to investigate whether the set of model parameters are unique. In other words, can any other set of model parameters lead to the same model output (Schwan, 2001). To answer this question, a sensitivity analysis is used. This is the study of the effect of variations in the model input parameters on the system behaviour or model output (Varma et al., 2005). In this way, the model developer can evaluate and gain an increased understanding regarding how each model parameter contributes to the model output. The strength and relevance of the parameter in determining the output can then be examined. This is important for model simplification because regions of model parameter insignificance and hence model output insensitivity can be identified (Schwan, 2001; Sharrock \& Coetzer, 2007).

Although it is often assumed that model parameters are independent of one another, correlation between model parameters occurs in almost all models to some degree (Kittrell, 1970). The consequence of parameter correlation is that complications are encountered by the regression procedure during parameter estimation (Sharrock \& Coetzer, 2007; Fry, 2010). This can be demonstrated by considering the rational function in Equation 5.1, where $a_{1}, a_{2}$ and $a_{3}$ are the model parameters (Sharrock \& Coetzer, 2007).

$$
f(x)=\frac{a_{1} x+a_{2}}{1+a_{3} x}
$$

It is important to consider the case where $a_{3} x \gg 1$. Equation 5.1 would then reduce to Equation 5.2 .

$$
f(x) \approx \frac{a_{1} x+a_{2}}{a_{3} x}=\frac{a_{1}}{a_{3}}+\frac{a_{2}}{a_{3}} \frac{1}{x}
$$

From Equation 5.2, the number of parameters has effectively been reduced from three to two, namely $\frac{a_{1}}{a_{3}}$ and $\frac{a_{2}}{a_{3}}$ Sharrock \& Coetzer 2007). However, any regression procedure will still 
encounter three model parameters. Consequently, the parameter estimation process may fail or the relationship between parameters rather than the actual parameter values may be regressed when fitting the model to data (Sharrock \& Coetzer, 2007; Fry, 2010). Hence, the estimated parameter values are incorrect and as such, are not going to be a measure of the validity of the model prediction (Fry, 2010).

This example highlights the importance of understanding the effect of the parameters $a_{1}, a_{2}$ and $a_{3}$ on the function behaviour over the full range of possible $x$ values. If parameters are correlated, it is best to reduce the number of model parameters that are required to be estimated (Schwan, 2001; Fry, 2010). This is done by fixing one of the parameters at a reasonable value e.g. a value of $a_{3}$ could be chosen such that the ratios $\frac{a_{1}}{a_{3}}$ and $\frac{a_{2}}{a_{3}}$ are preserved (Sharrock \& Coetzer, 2007). Alternatively, the model can be reformulated, where the form given in Equation 5.2, using two parameters is used instead.

A major benefit of performing a sensitivity analysis is that it can also reveal the behaviour encountered in the above example. It is thus advantageous to perform a sensitivity analysis because it can simplify the model by allowing over-parametrisation to be identified. This will provide increased understanding between the model input and model output, can save computation time during the parameter estimation procedure and enhance the confidence in the resulting parameter estimates and model formulation.

\subsection{Sensitivity Analysis Approaches}

To investigate the effect of the rate constants $k_{1}-k_{6}$, the model parameters, on the selectivity to $\mathrm{C}_{1}, \mathrm{C}_{2}, \mathrm{C}_{3}, \mathrm{C}_{4}, \mathrm{C}_{5+}$ and $\mathrm{CO}_{2}$, two approaches will be used. These are the one-at-a-time and the derivative-based local approaches respectively and are outlined below in terms of the insight that they provide. The goal of the sensitivity analysis is to determine whether the rate constants represent a unique set and if it is possible to determine meaningful values of them that would accurately describe experimental data upon regression.

\subsubsection{One-at-a-time Approach}

The one-at-a-time approach is a common method, which is both simple and practical to investigate the effect that changing a rate constant has on the model output e.g. product selectivity. It has been successfully applied in the development of the propene oligomerisation kinetic model of Sealy (1996).

This approach involves changing one rate constant at a time by varying it by an order of magnitude 
relative to its base case value while keeping the values of the other rate constants fixed at their base case values. The varied rate constant is then returned to its base case value and the process is repeated for the other rate constants in the same way.

The variation of the rate constants in this way ensures that any observable change in the model output is directly linked to the change in rate constant. The results are easy to compare because they have all been evaluated with respect to the same reference point i.e. the base case. It is an effective tool to study the reaction pathway. A shortcoming, however, is that correlation between model parameters cannot be detected.

\subsubsection{Derivative-Based Local Approach}

The derivative-based local approach to sensitivity analysis involves taking the partial derivative of the product selectivity with respect to each rate constant. This is the first order or local absolute sensitivity of the product selectivity, defined by Equation 5.3 (Varma et al., 2005).

$$
\frac{\partial S_{i}}{\partial k_{j}}=\lim _{\Delta k_{j} \rightarrow 0} \frac{S_{i}\left(k_{j}+\Delta k_{j}\right)-S_{i}\left(k_{j}\right)}{\Delta k_{j}}
$$

For more effective comparison the magnitude of all sensitivities are normalised. This is essential because the value of the product selectivity and rate constants can differ substantially by many orders of magnitude (Schwan, 2001). This is the relative sensitivity of the product selectivity defined by Equation 5.4 (Varma et al., 2005).

$$
\frac{\partial \ln \left(S_{i}\right)}{\partial \ln \left(k_{j}\right)}=\frac{k_{j}}{S_{i}} \frac{\partial S_{i}}{\partial k_{j}}
$$

Plots of the relative sensitivity of the product selectivity as a function of $\mathrm{CO}$ conversion are a useful tool to compare the effect of each rate constant on the product selectivity and provide insight regarding the experimental conditions necessary for an accurate parameter estimation (Schwan, 2001). Over-parametrisation of the model can also easily be identified if sensitivities are zero or if sensitivities are linearly dependent.

The work of Schwan (2001) provides an approach to test for the linear dependence of relative sensitivity functions when only three parameters are involved. A test for linear dependence is conducted by fitting a straight line to the ratio of relative sensitivity functions $s_{i, j}\left(X_{C O}\right)$ for $j=$ $1,2,3$ in Equation 5.5 .

$$
\frac{s_{i, 3}\left(X_{C O}\right)}{s_{i, 2}\left(X_{C O}\right)}=a_{i}+b_{i} \frac{s_{i, 1}\left(X_{C O}\right)}{s_{i, 2}\left(X_{C O}\right)}
$$


where

$$
s_{i, j}\left(X_{C O}\right)=\frac{\partial \ln \left(S_{i}\right)}{\partial \ln \left(k_{j}\right)}
$$

The degree of linearity can quantified using the correlation coefficient ${ }^{1} R^{2}$. Linearity can be confirmed if $R^{2} \rightarrow 1$. In other words, if $R^{2}$ is close to one, then model parameters are correlated and the model is thus over-parametrised (Schwan, 2001). This represents a simple and computationally lightweight approach to investigating the linear dependence between model parameters.

\subsection{One-at-a-time Analysis}

The purpose of this section is to illustrate and discuss the effect of the rate constants, $k_{1}-k_{6}$, on the selectivity to $\mathrm{C}_{1}, \mathrm{C}_{2}, \mathrm{C}_{3}, \mathrm{C}_{4}, \mathrm{C}_{5+}$ and $\mathrm{CO}_{2}$ as a function of $\mathrm{CO}$ conversion. In other words, does each model parameter have a distinct effect on product selectivity, producing different selectivity profiles over the measured $\mathrm{CO}$ conversion range? Furthermore, valid insight can be obtained regarding the reaction pathway.

The base case rate constant values are given in Table 5.1. Each rate constant is varied one at a time while the values of the other rate constants are held fixed at their values given in Table 5.1. The effect of the varied rate constant on the carbon-based selectivity is studied up to a CO conversion of $95 \%$. This is chosen based on the highest CO conversion measured in the experimental studies of Tucker \& van Steen (2019). The effect of the varied rate constant $k_{j}$ is then studied at $0.01 k_{j}$, $0.1 k_{j}, k_{j}, 10 k_{j}$ and $100 k_{j}(j=1,2, \ldots, 6)$. These analyses are carried out at a temperature of $220^{\circ} \mathrm{C}$, total pressure of $20 \operatorname{bar}(\mathrm{a})$ and $\mathrm{H}_{2} / \mathrm{CO}$ feed ratio of 2.1 .

Table 5.1: Numerical values of the base case rate constants in reaction step 1-6

\begin{tabular}{cc}
\hline Rate constant & Value $[\mathrm{mol} / \mathrm{kg} / \mathrm{s}]$ \\
\hline$k_{1}$ & 0.0001 \\
$k_{2}$ & 0.001 \\
$k_{3}$ & 0.1 \\
$k_{4}$ & 10 \\
$k_{5}$ & 1 \\
$k_{6}$ & 0.0001 \\
\hline
\end{tabular}

\footnotetext{
${ }^{1} R^{2}$ is not a measure of the 'goodness' of fit but rather provides a measure of the degree of linear correlation between the data and the model prediction.
} 


\subsubsection{Varying $\mathbf{k}_{1}$}

Variations in $k_{1}$ result in a significant variation in the selectivity versus $C O$ conversion curves for $C_{1}$, $\mathrm{C}_{2}, \mathrm{C}_{3}, \mathrm{C}_{4}, \mathrm{C}_{5+}$ and $\mathrm{CO}_{2}$ in Figure 5.1 and 5.2 . This is because $\mathrm{k}_{1}$ controls the rate of monomer formation and hence directly influences the rate of the methanation, chain growth and water gas shift reactions. For a slow rate of monomer formation $\left(0.01 k_{1}\right)$, the major products are methane and $\mathrm{C}_{2}$ at low $\mathrm{CO}$ conversion, changing to methane and carbon dioxide as $\mathrm{CO}$ conversion increases. Chain growth leading to the formation of long chain hydrocarbons is unfavourable and limited because of the slow rate of formation of the monomer, $\mathrm{CH}_{2}$ and its preferential hydrogenation to methane. The high $\mathrm{CO}_{2}$ selectivity is the result of the water gas shift rate being two orders of magnitude greater than the $\mathrm{FT}$ reaction rate. This results in a high $\mathrm{H}_{2}$ content favouring hydrogenation of the monomer. Hence, the major hydrocarbon product corresponds to methane. This appears to be a more consistent description of methanation catalysis.

With increases in $k_{1}$, the selectivity to long chain hydrocarbons becomes more favourable as the rate of monomer formation is greater favouring chain growth. This is shown by an increase in selectivity to longer chain hydrocarbons. With increases in $k_{1}$ and hence the rate of monomer formation, the selectivity to methane and $\mathrm{CO}_{2}$ decreases over the $\mathrm{CO}$ conversion range simulated in Figure 5.1 and 5.2. Conversely, the selectivity to $C_{5+}$ increases with increases in $k_{1}$. There is also an increase in the maximum selectivity to $\mathrm{C}_{5+}$, which shifts to a higher $\mathrm{CO}$ conversion range with increases in $k_{1}$. This is the result of greater carbon utilisation as most of the carbon in CO reacts to form long chain hydrocarbons. This indicates that the magnitude of $k_{1}$ controls not just the rate of monomer formation but the rate of the chain growth reactions as well. This is consistent with $\mathrm{CO}$ hydrogenation being the rate-determining reaction step, which highlights its importance during the FT reactions.

From the base case (red curves), it is noted that the primary products correspond to methane, ethane and ethene at low CO conversion. This is observed by the immediate selectivity to $C_{1}$ and $C_{2}$ in Figure 5.1 and 5.2. This is since these hydrocarbon products are the first to form and thus account for and constitute the carbon-based selectivity at low CO conversion. With increases in CO conversion, there is an increase in $C_{3}, C_{4}$ and $C_{5+}$ selectivity, with a decrease in selectivity to the primary products as chain growth becomes favoured. There is thus a shift from low carbon number to high carbon number products with increases in $\mathrm{CO}$ conversion as $\mathrm{C}_{5+}$ selectivity becomes favoured. Once the maximum $C_{5+}$ selectivity has been reached, the reaction path shifts back to favouring products of low carbon number. As such there is a rise in the selectivity to methane, $C_{2}$, $C_{3}$ and $C_{4}$. This is consistent with the reverse reaction in the n-paraffin and 1-olefin homologous series being favoured, which will eventually lead to the large thermodynamic driving force that exists 
to form methane at complete chemical equilibrium. The steady rise in $\mathrm{CO}_{2}$ selectivity is a result of the reaction only being able to occur once water forms. The WGS reaction is thus also limited by $\mathrm{CO}$ hydrogenation in the $\mathrm{FT}$ reaction system.

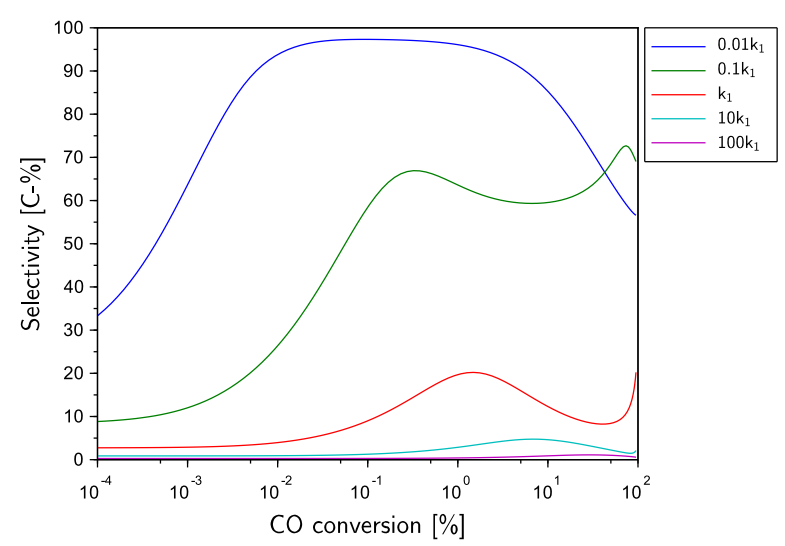

(a) Selectivity to $C_{1}$

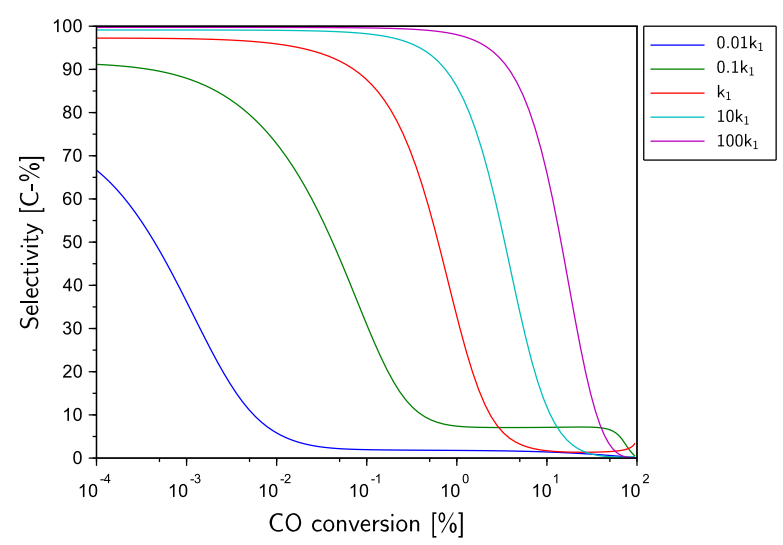

(c) Selectivity to $C_{2}$

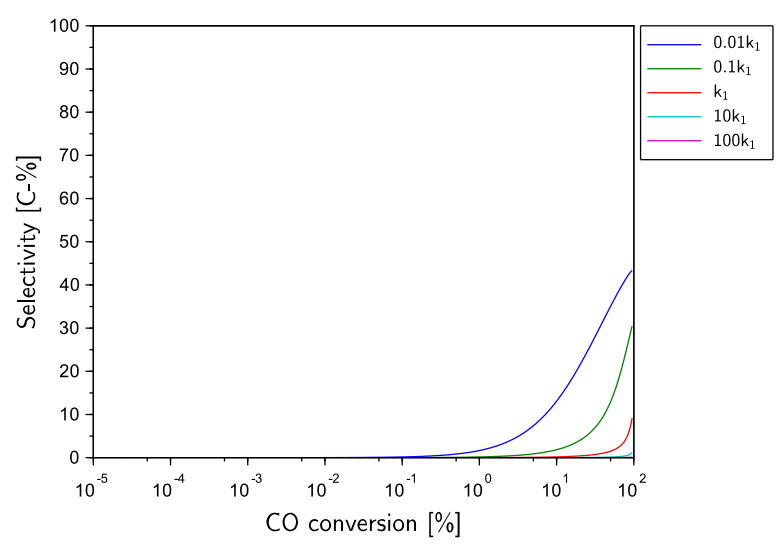

(b) Selectivity to $\mathrm{CO}_{2}$

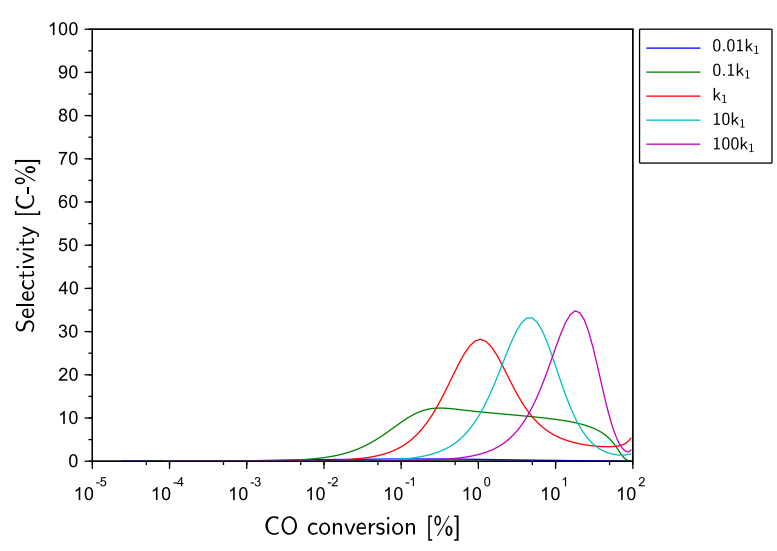

(d) Selectivity to $C_{3}$

Figure 5.1: The effect of variations of $k_{1}$ on the carbon-based selectivity at $220^{\circ} \mathrm{C}, 20 \mathrm{bar}(\mathrm{a})$ and $\mathrm{H}_{2} / \mathrm{CO}$ feed ratio of 2.1 


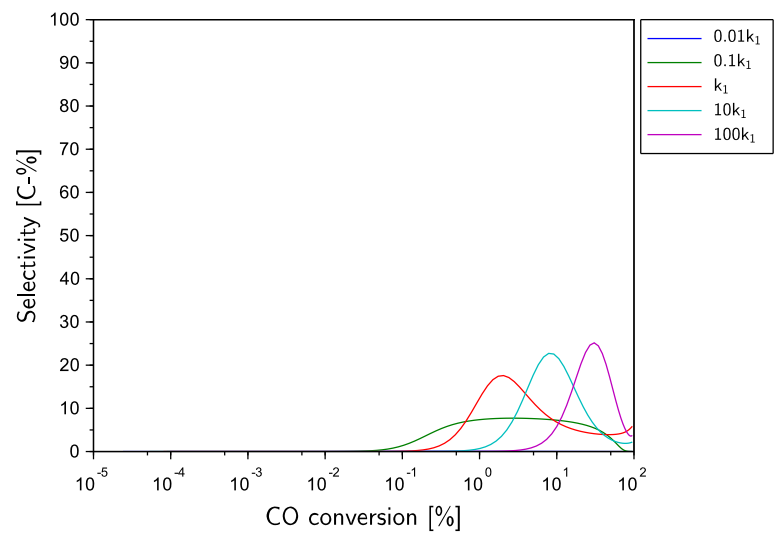

(a) Selectivity to $\mathrm{C}_{4}$

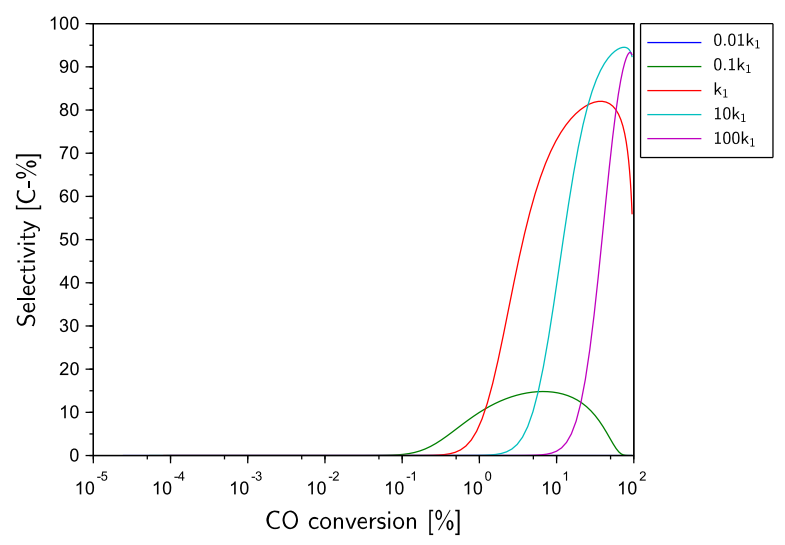

(b) Selectivity to $\mathrm{C}_{5+}$

Figure 5.2: The effect of variations of $k_{1}$ on the carbon-based selectivity at $220^{\circ} \mathrm{C}, 20$ bar(a) and $\mathrm{H}_{2} / \mathrm{CO}$ feed ratio of 2.1 continued

\subsubsection{Varying $\mathrm{k}_{2}$}

With increases in $\mathrm{k}_{2}$, there is an increase in the selectivity to methane and $\mathrm{CO}_{2}$ in Figure 5.3 . This corresponds to a decrease in the selectivity to $C_{5+}$ with increases in $k_{2}$. This indicates that the monomer is preferentially consumed in the methanation reaction rather than the chain growth reactions. The increase in $\mathrm{CO}_{2}$ selectivity with increases in $\mathrm{k}_{2}$ is due to the increased $\mathrm{H}_{2}$ consumption, favouring the forward WGS reaction. This arises due to the rapid consumption of $\mathrm{CH}_{2}$ by the methanation reaction, favouring the rate of the forward WGS reaction leading to $\mathrm{CO}_{2}$ formation. With an increase in the methanation rate, selectivity to $C_{2}, C_{3}$ and $C_{4}$ all increase over a certain $\mathrm{CO}$ conversion range up to the base case value of $\mathrm{k}_{2}$, corresponding to the decrease in $C_{5+}$ selectivity. Further increases in $k_{2}$ forces the selectivity to $C_{2}, C_{3}$ and $C_{4}$ to tend to zero close to complete conversion to favour selectivity towards $C_{1}$, the most thermodynamically favoured product. This highlights the importance of minimising the rate of methane formation to ensure favourable $\mathrm{C}_{5+}$ selectivity can be obtained. This can be observed for variations in $\mathrm{k}_{2}$ up to two orders of magnitude below its base case value where there is a rise in the $C_{5+}$ selectivity curves in Figure 5.3 . 


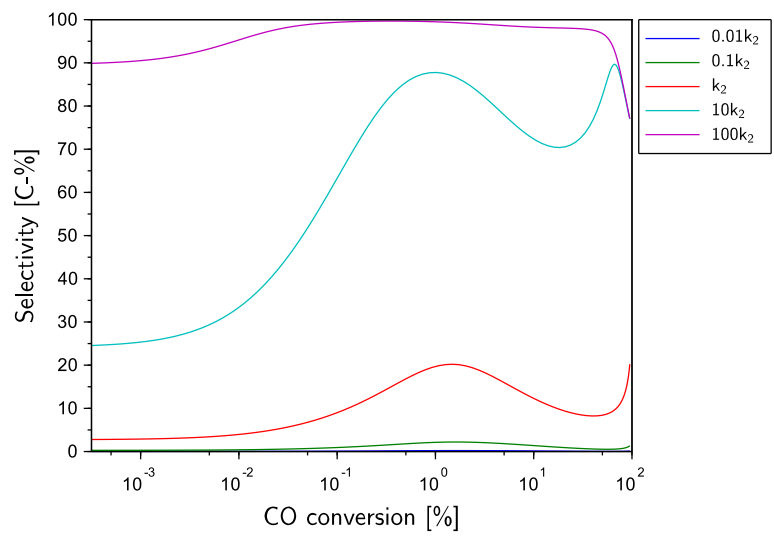

(a) Selectivity to $C_{1}$

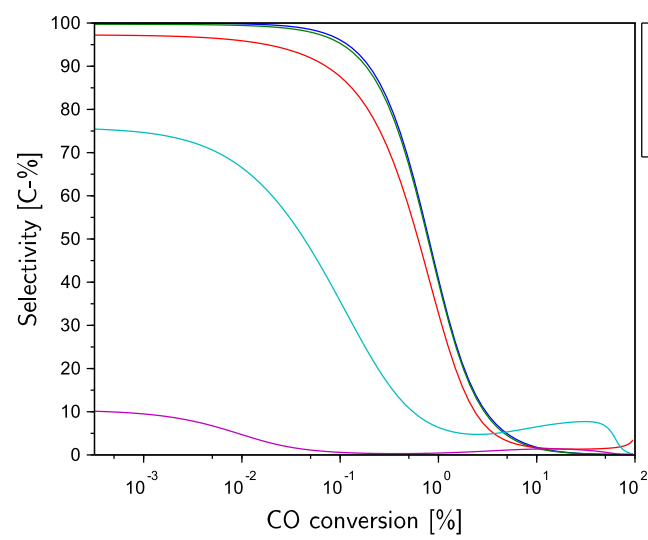

(c) Selectivity to $\mathrm{C}_{2}$

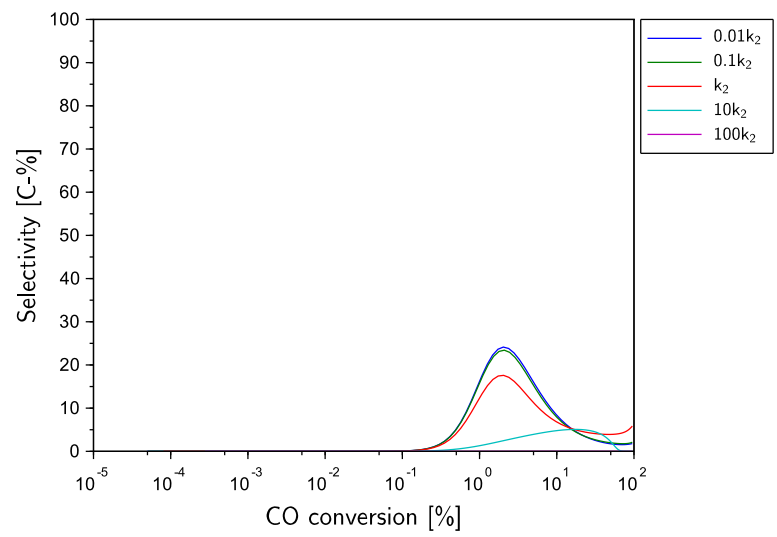

(e) Selectivity to $C_{4}$

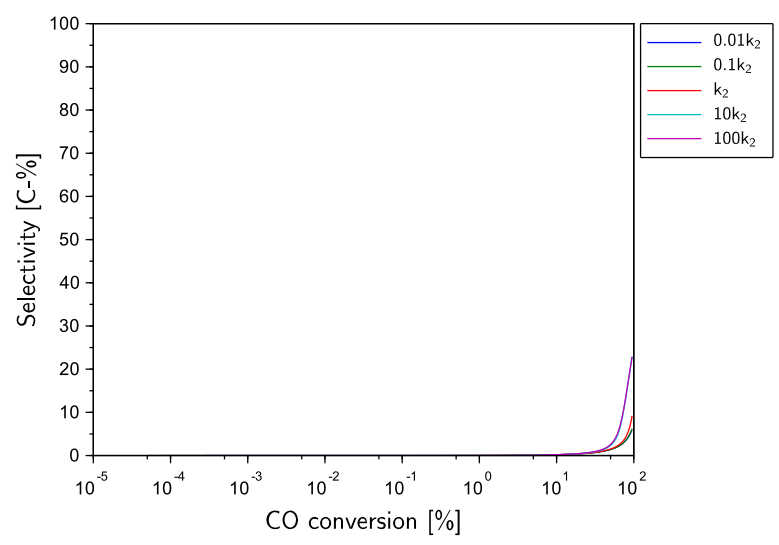

(b) Selectivity to $\mathrm{CO}_{2}$

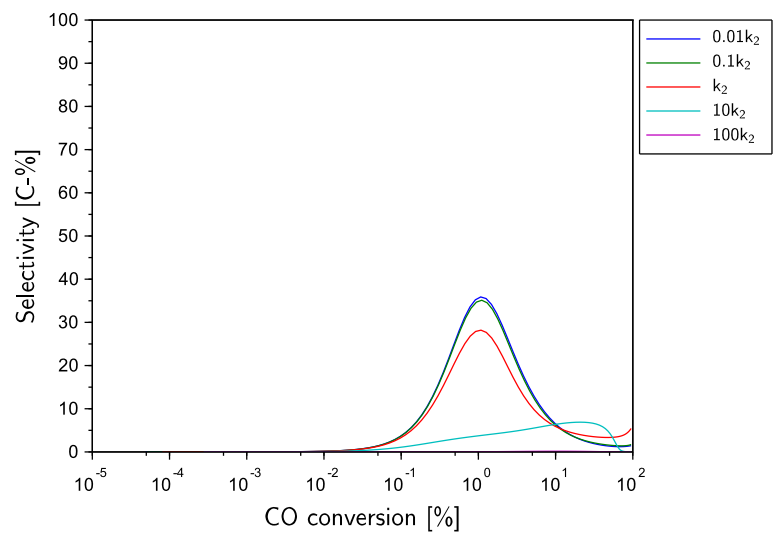

(d) Selectivity to $C_{3}$

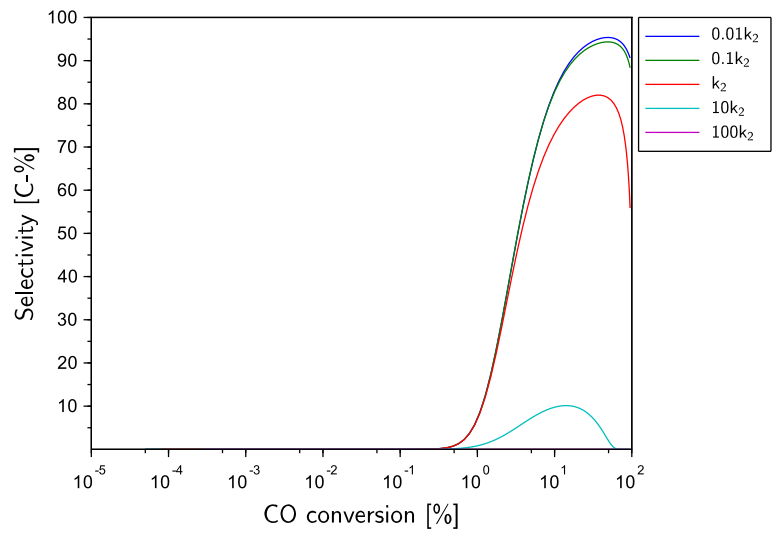

(f) Selectivity to $\mathrm{C}_{5+}$

Figure 5.3: The effect of variations of $k_{2}$ on the carbon-based selectivity at $220^{\circ} \mathrm{C}, 20 \mathrm{bar}(\mathrm{a})$ and $\mathrm{H}_{2} / \mathrm{CO}$ feed ratio of 2.1 


\subsubsection{Varying $\mathrm{k}_{3}$}

Variations in $k_{3}$ have the most notable effect on the selectivity to $C_{1}, C_{3}, C_{4}$, and $C_{5+}$ in Figure 5.4 and 5.5. The selectivity to $\mathrm{C}_{2}$ and $\mathrm{CO}_{2}$ over the simulated $\mathrm{CO}$ conversion range yields virtually the same set of curves despite variations in $k_{3}$. This indicates that the selectivity to $C_{2}$ is virtually insensitive to changes in $k_{3}$. The function of $k_{3}$ is to initiate chain growth to $n$-paraffins of higher carbon number beginning at methane. The selectivity to $C_{2}$ over the simulated $\mathrm{CO}$ conversion range therefore appears insensitive to increases in $k_{3}$ because the faster $C_{2}$ forms, specifically ethane, the faster it is consumed to form higher carbon number n-paraffins. This results in the observed insensitivity in Figure 5.4 and 5.5. The selectivity to $\mathrm{CO}_{2}$ curves over the simulated $\mathrm{CO}$ conversion range indicates that $\mathrm{CO}_{2}$ formation is unaffected by the rate of initiation. Increases in $\mathrm{k}_{3}$ therefore lead to the observed decrease in methane selectivity as the initiation reaction rate increases. This favours chain growth, which is observed as the increase in $C_{3}, C_{4}$ and $C_{5+}$ selectivity curves.

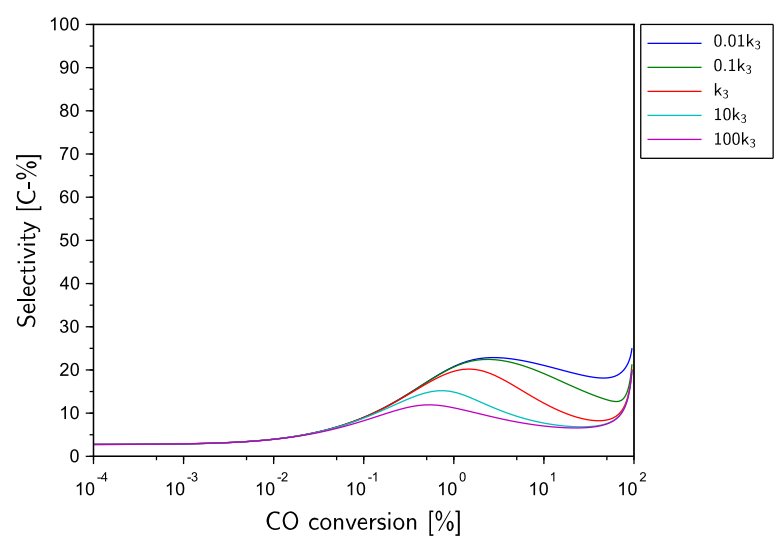

(a) Selectivity to $C_{1}$

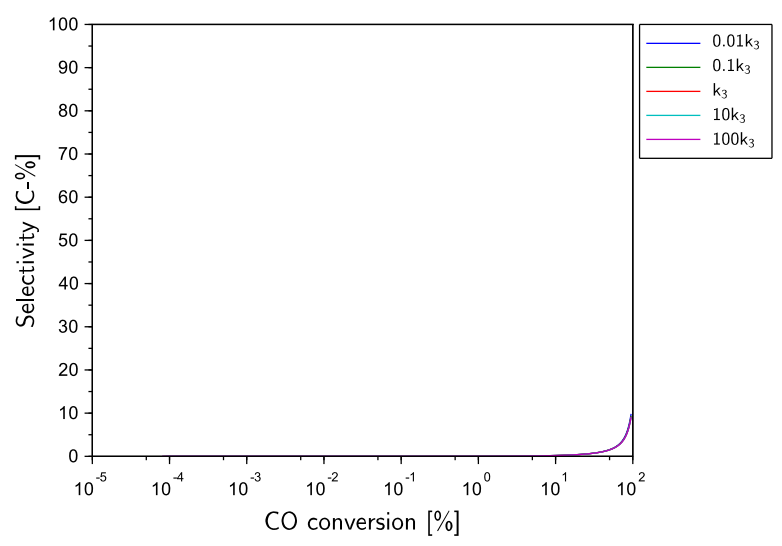

(b) Selectivity to $\mathrm{CO}_{2}$

Figure 5.4: The effect of variations of $k_{3}$ on the carbon-based selectivity at $220^{\circ} \mathrm{C}, 20$ bar(a) and $\mathrm{H}_{2} / \mathrm{CO}$ feed ratio of 2.1 


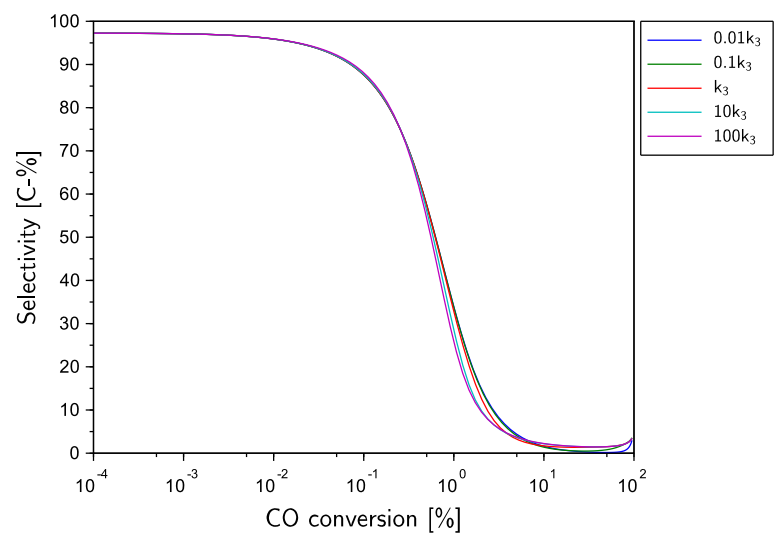

(a) Selectivity to $C_{2}$

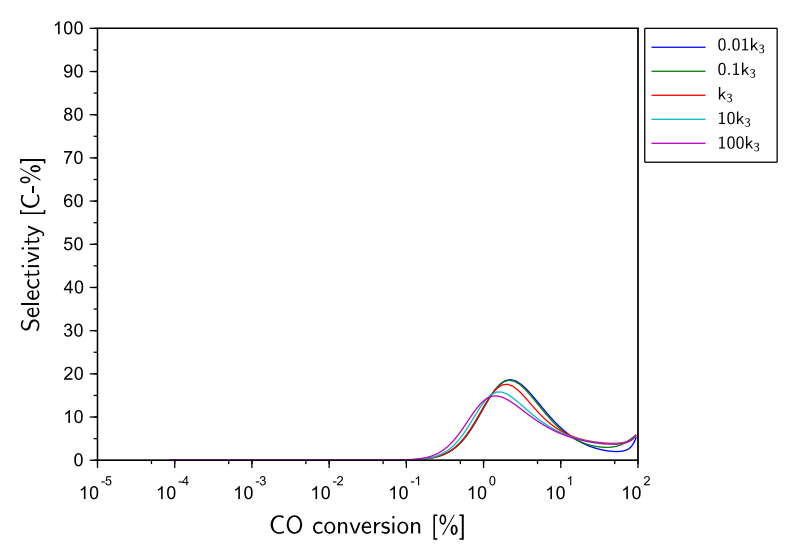

(c) Selectivity to $\mathrm{C}_{4}$

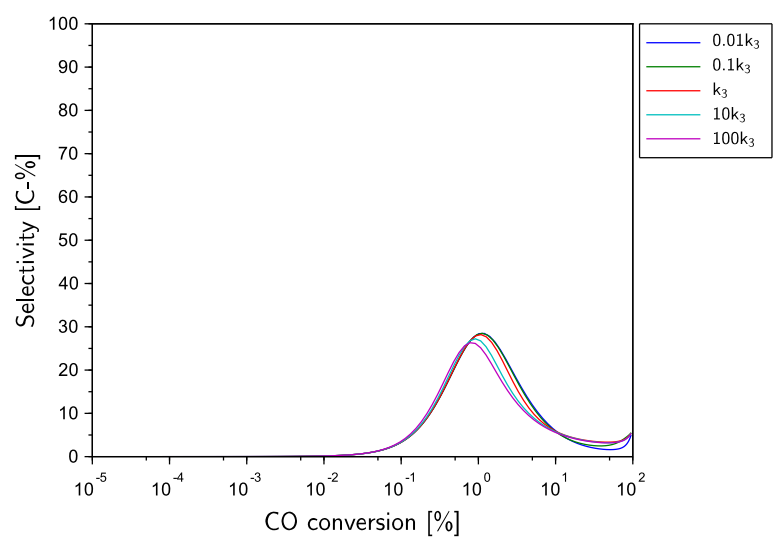

(b) Selectivity to $C_{3}$

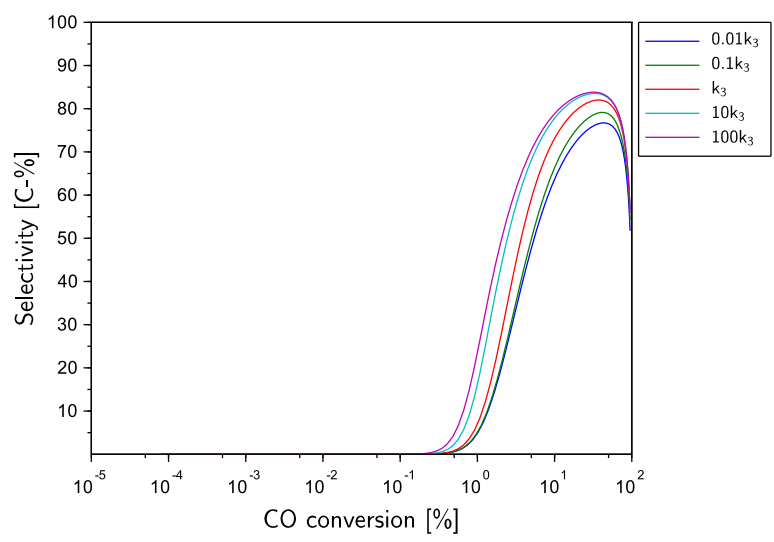

(d) Selectivity to $\mathrm{C}_{5+}$

Figure 5.5: The effect of variations of $k_{3}$ on the carbon-based selectivity at $220^{\circ} \mathrm{C}, 20$ bar(a) and $\mathrm{H}_{2} / \mathrm{CO}$ feed ratio of 2.1 continued

\subsubsection{Varying $\mathrm{k}_{4}$}

Variations in $k_{4}$ affects the selectivity to $C_{1}, C_{2}, C_{3}, C_{4}$ and $C_{5+}$ in Figure 5.6 and 5.7. This is because it controls the rate of chain growth for the n-paraffins. The selectivity curves to $C_{1}, C_{2}$, $\mathrm{C}_{3}$ and $\mathrm{C}_{4}$ all decrease with increasing $\mathrm{k}_{4}$, at least up to a $\mathrm{CO}$ conversion of $10^{1 \%} \%$. As a result, the selectivity to $\mathrm{C}_{5+}$ curves all increase with increasing $\mathrm{k}_{4}$, also at least up to a $\mathrm{CO}$ conversion of $10^{1 \%}$. This increase can be explained by the preferential consumption of $C_{1}-C_{4} n$-paraffins to form $\mathrm{C}_{5+} \mathrm{n}$-paraffins in the $\mathrm{n}$-paraffin chain growth reactions. This is due to the series nature of the $n$-paraffin chain growth reactions, with an increase in $k_{4}$ favouring chain growth i.e. the forward reaction. However, the equilibrium constant for the formation of $n$-paraffins of carbon number $\mathrm{C}_{3}$ and greater are all equal to unity. As such, the forward and reverse rate constants are equal. An increase in $k_{4}$ therefore results in the reverse $n$-paraffin chain growth reactions being favoured 
equally. This results in a shift back to lower carbon number products at lower CO conversions. This is seen by the decrease in the selectivity to $C_{5+}$ with increases in the value of $k_{4}$ leading to an increase in the selectivity to $C_{1}, C_{2}, C_{3}$ and $C_{4}$ for $C O$ conversions greater than $10^{1} \%$ in Figure 5.6 and 5.7. The selectivity to $\mathrm{CO}_{2}$ curves appears to be insensitive to changes in $\mathrm{k}_{4}$. This indicates that $\mathrm{CO}_{2}$ formation is unaffected by the rate constant $\mathrm{k}_{4}$.

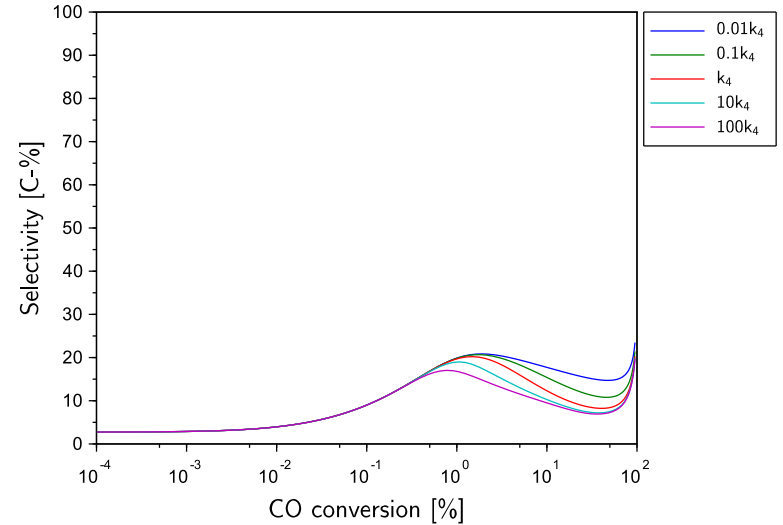

(a) Selectivity to $C_{1}$

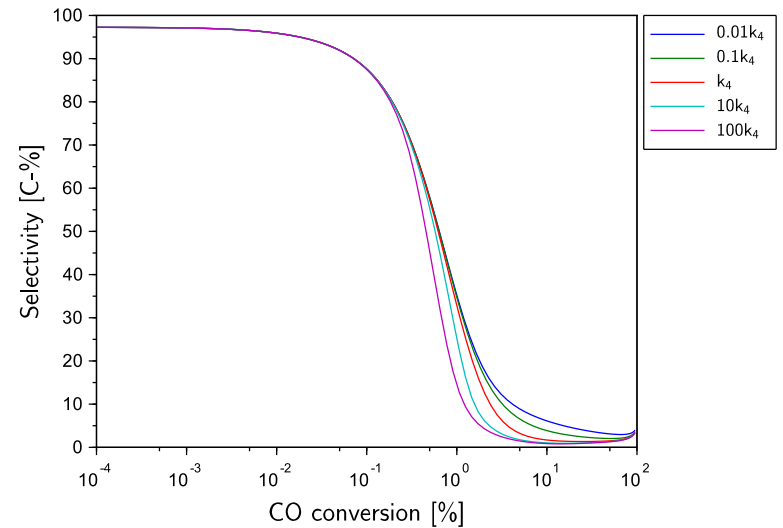

(c) Selectivity to $C_{2}$

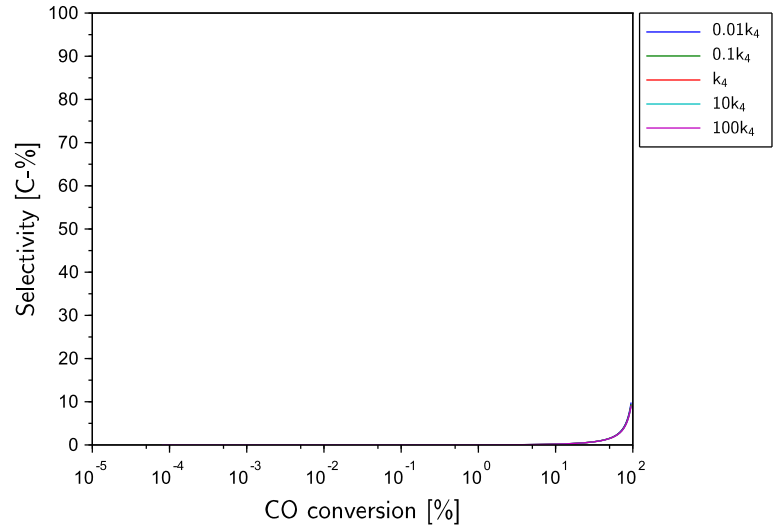

(b) Selectivity to $\mathrm{CO}_{2}$

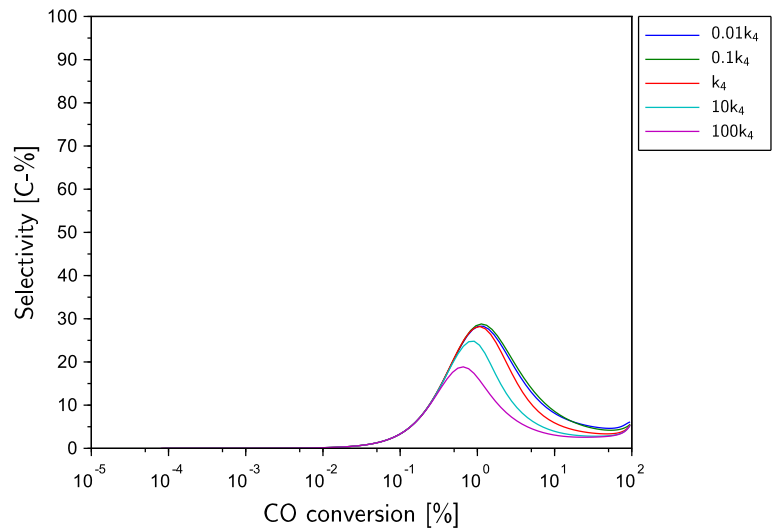

(d) Selectivity to $C_{3}$

Figure 5.6: The effect of variations of $k_{4}$ on the carbon-based selectivity at $220^{\circ} \mathrm{C}, 20$ bar(a) and $\mathrm{H}_{2} / \mathrm{CO}$ feed ratio of 2.1 


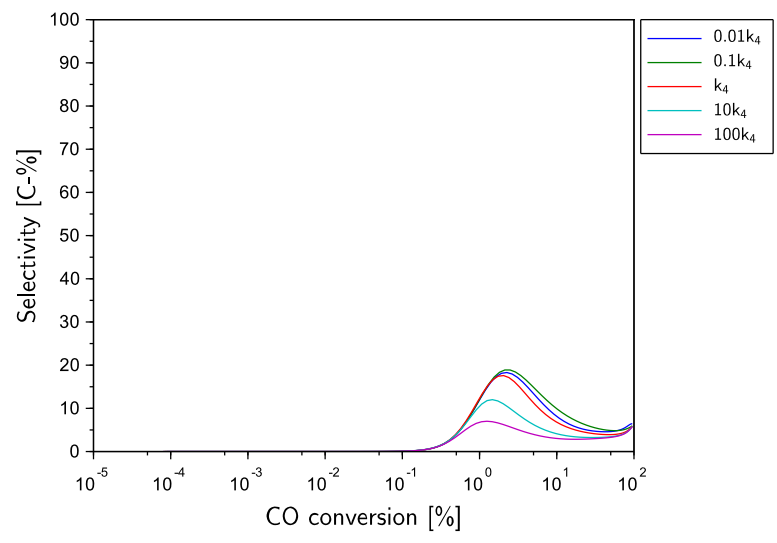

(a) Selectivity to $C_{4}$

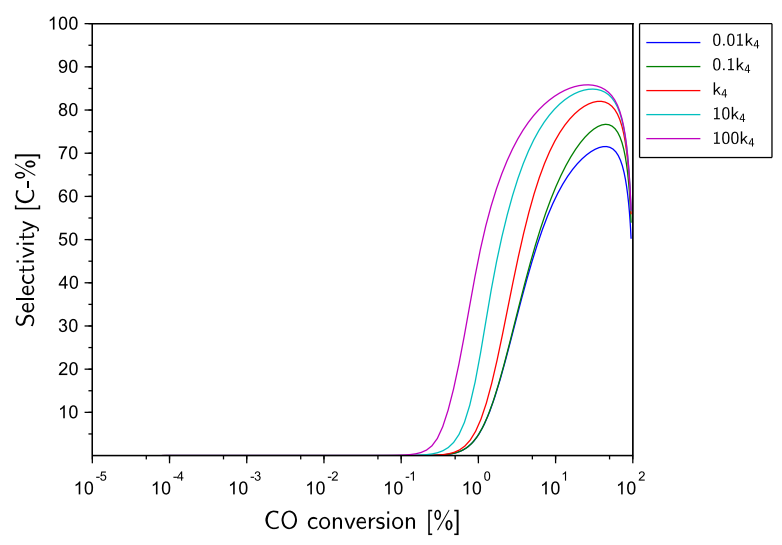

(b) Selectivity to $\mathrm{C}_{5+}$

Figure 5.7: The effect of variations of $k_{4}$ on the carbon-based selectivity at $220^{\circ} \mathrm{C}, 20$ bar(a) and $\mathrm{H}_{2} / \mathrm{CO}$ feed ratio of 2.1 continued

\subsubsection{Varying $k_{5}$}

Variations in $k_{5}$ affect the selectivity of $C_{1}, C_{2}, C_{3}, C_{4}$ and $C_{5+}$ most notably across the CO conversion range simulated in Figure 5.8. For $\mathrm{CO}$ conversions less than $1 \%$, there is a shift in the primary selectivity from mainly $C_{1}$ to mainly $C_{2}$. This arises because of increase in the rate of $\mathrm{CH}_{2}$ coupling. Thus, with increases in both $\mathrm{CO}$ conversion and $\mathrm{k}_{5}$, an increase in the $\mathrm{C}_{3}$ and $C_{4}$ selectivity curves is observed. The $C_{3}$ and $C_{4}$ product range is also where the 1-olefin content is greatest. Therefore the selectivity to $C_{3}$ and $C_{4}$ curves show the most notable increase with increases in $k_{5}$ since propene and 1-butene account for most of the selectivity at these carbon numbers. For $\mathrm{CO}$ conversions of $10 \%$ and greater the $\mathrm{C}_{2}$ selectivity curves appear unchanged because of the low thermodynamic driving force to form ethene and its consumption to form longer chain 1-olefins. The increase in selectivity to $C_{3}$ and $C_{4}$ with increases in $k_{5}$ corresponds to a decrease in $\mathrm{C}_{5+}$ selectivity. This is due to the promotion of the rate of formation of 1-olefins of low carbon numbers. A decrease in the $C_{1}$ selectivity curves is observed with increases in $k_{5}$ at least until a CO conversion of $50 \%$. Thereafter, the $C_{1}$ selectivity curves all rise. The reverse 1-olefin chain growth reaction is favoured, leading to an increase in $\mathrm{CH}_{2}$ formation and hence its hydrogenation to methane. This leads to the increase in $C_{1}$ selectivity in Figure 5.8. This indicates that faster 1-olefin chain growth enables better carbon utilisation as $\mathrm{CO}$ is preferentially hydrogenated to $\mathrm{CH}_{2}$. From Figure $5.8, \mathrm{CO}_{2}$ formation is unaffected by the rate constant $\mathrm{k}_{5}$. 


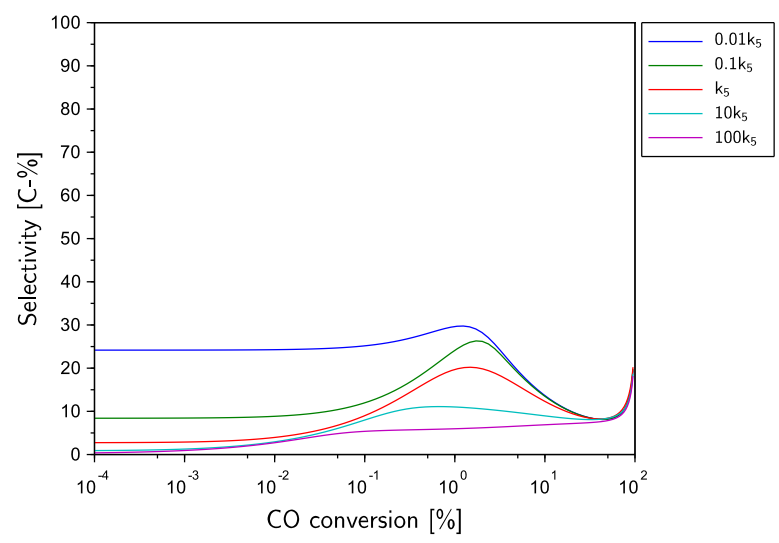

(a) Selectivity to $C_{1}$

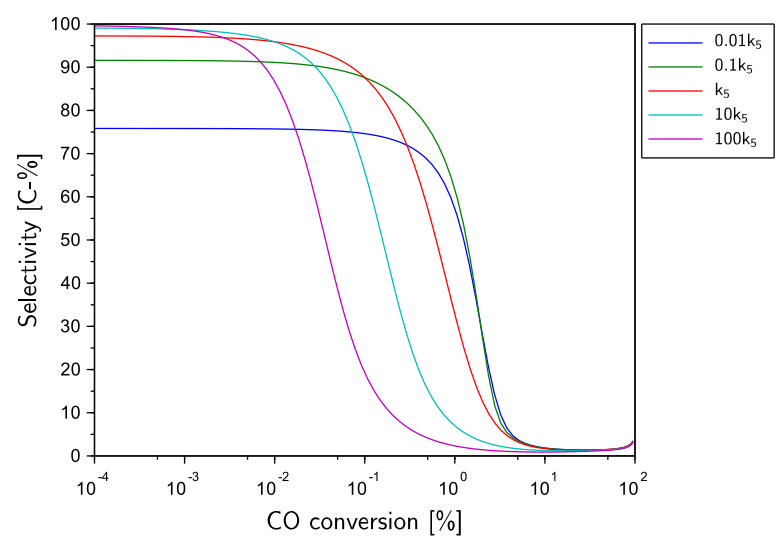

(c) Selectivity to $C_{2}$

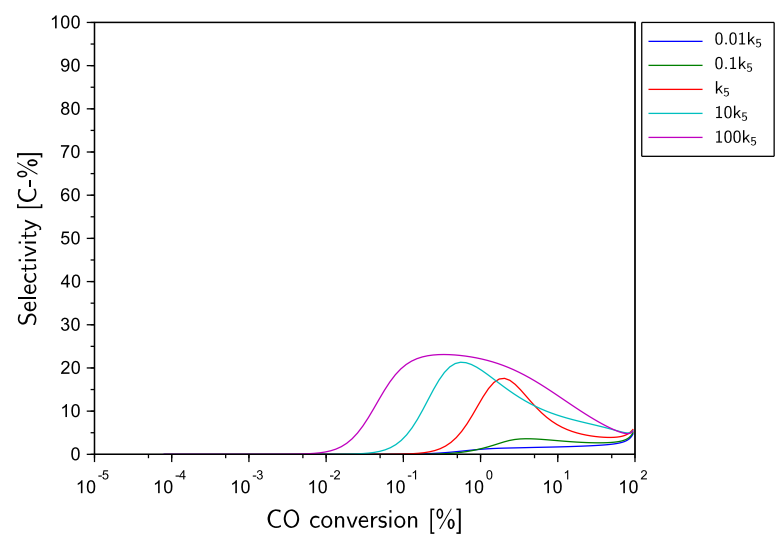

(e) Selectivity to $C_{4}$

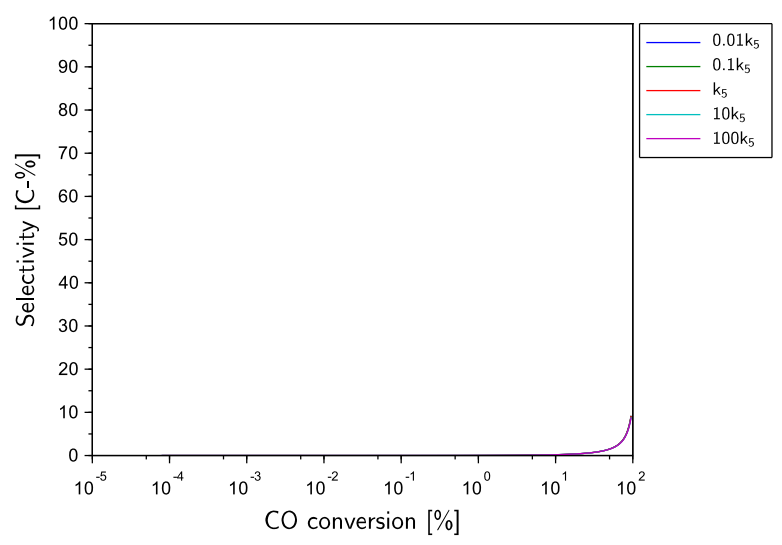

(b) Selectivity to $\mathrm{CO}_{2}$

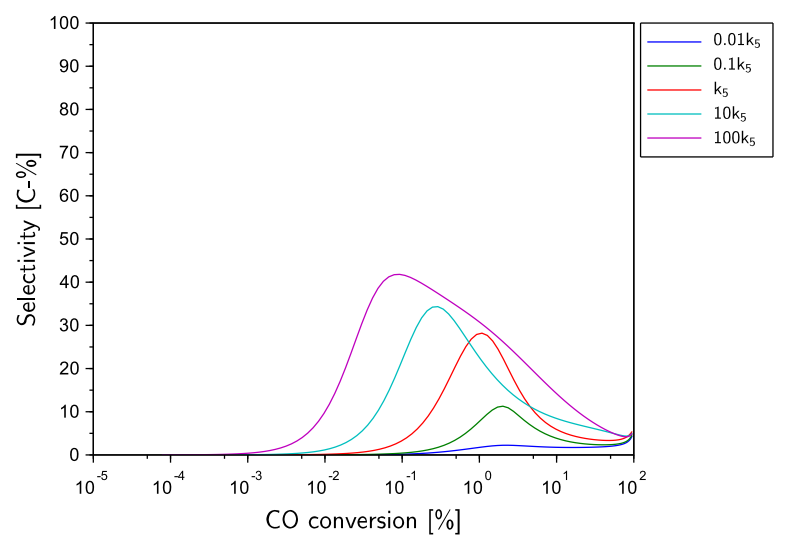

(d) Selectivity to $C_{3}$

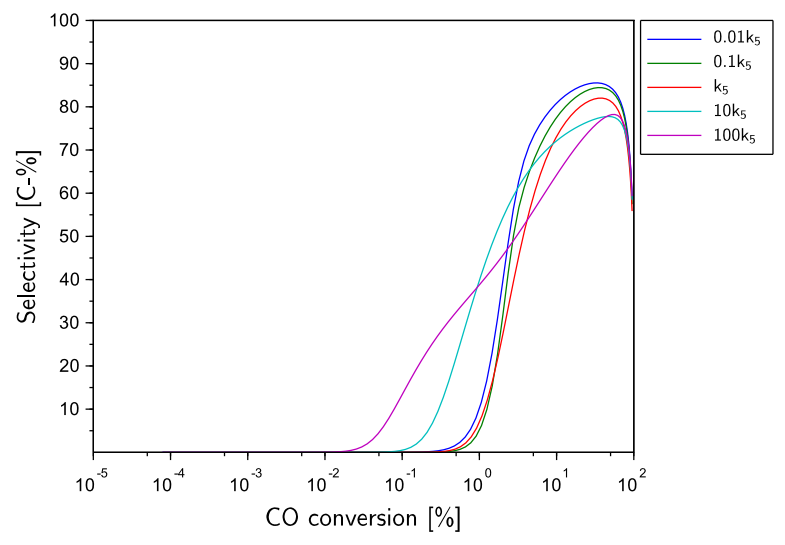

(f) Selectivity to $\mathrm{C}_{5+}$

Figure 5.8: The effect of variations of $k_{5}$ on the carbon-based selectivity at $220^{\circ} \mathrm{C}, 20 \mathrm{bar}(\mathrm{a})$ and $\mathrm{H}_{2} / \mathrm{CO}$ feed ratio of 2.1 


\subsubsection{Varying $\mathrm{k}_{6}$}

Variations in $\mathrm{k}_{6}$ affects the selectivity of $\mathrm{C}_{1}, \mathrm{C}_{2}, \mathrm{C}_{3}, \mathrm{C}_{4}, \mathrm{C}_{5+}$ and $\mathrm{CO}_{2}$ only once the $\mathrm{CO}$ conversion is greater than $10^{1} \%$ in Figure 5.9 and 5.10 . This is expected since the WGS reaction can only occur once water forms and as such it is limited by $\mathrm{CO}$ hydrogenation. This indicates that the rate constant $\mathrm{k}_{1}$ has a stronger influence in determining the selectivity to $\mathrm{CO}_{2}$ than $\mathrm{k}_{6}$. Nonetheless, once the CO conversion is above $10^{1} \%$, with increases in $k_{6}$, the selectivity to $C_{3}$ and $C_{4}$ curves decrease, while the selectivity to $C_{2}$ curves remains unchanged. The initial decrease in $C_{3}$ and $\mathrm{C}_{4}$ selectivity curves corresponds to increases in selectivity to $C_{5+}$ up to $k_{6}$ being one order of magnitude below the base case, before decreasing $C_{5+}$ selectivity leads to increasing $C_{3}$ and $C_{4}$ selectivity. The increase in the WGS reaction rate relative to the $\mathrm{FT}$ reaction rate results in $\mathrm{CO}$ preferentially reacting to form $\mathrm{CO}_{2}$ rather than being hydrogenated to $\mathrm{CH}_{2}$. This results in the observed increase in the selectivity to $\mathrm{CO}_{2}$ curves with increasing $\mathrm{k}_{6}$ and the reduced hydrocarbon selectivity due to reduced chain growth. Although, again CO can only react along the WGS reaction pathway once water forms.

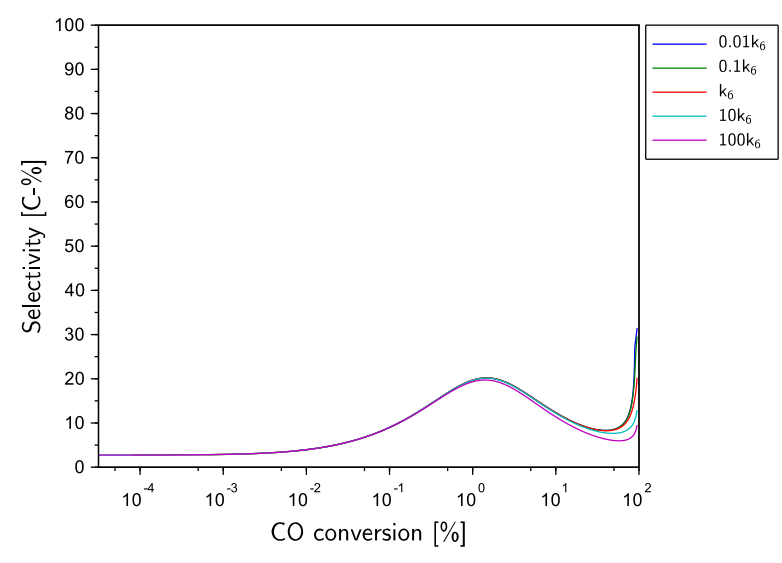

(a) Selectivity to $C_{1}$

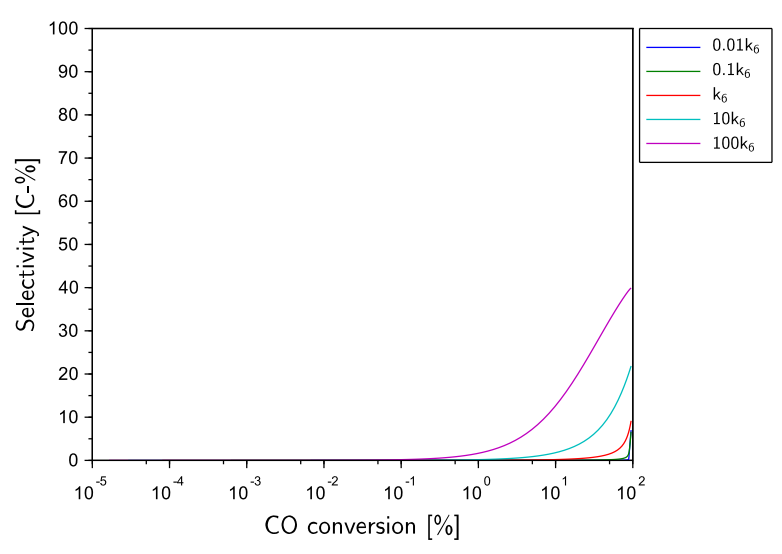

(b) Selectivity to $\mathrm{CO}_{2}$

Figure 5.9: The effect of variations of $k_{6}$ on the carbon-based selectivity at $220^{\circ} \mathrm{C}, 20$ bar(a) and $\mathrm{H}_{2} / \mathrm{CO}$ feed ratio of 2.1 


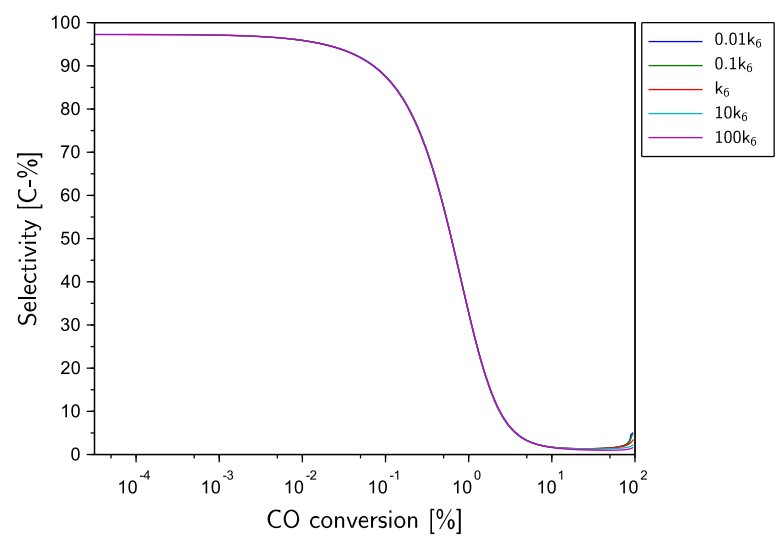

(a) Selectivity to $C_{2}$

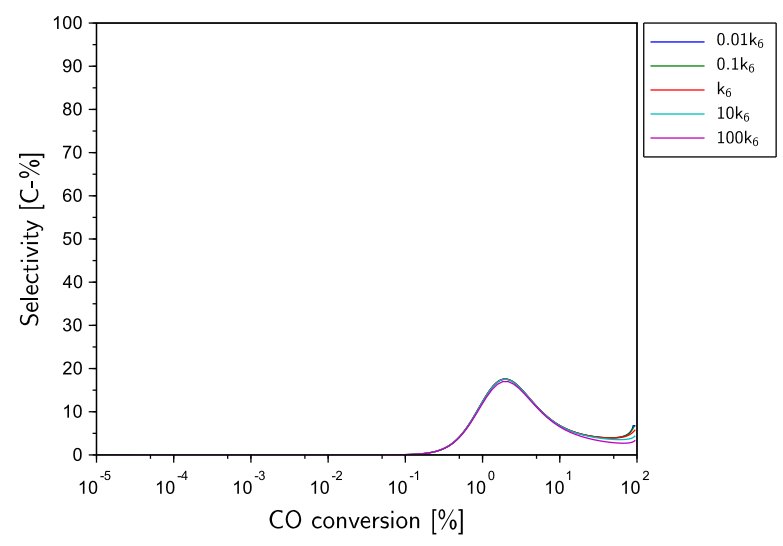

(c) Selectivity to $\mathrm{C}_{4}$

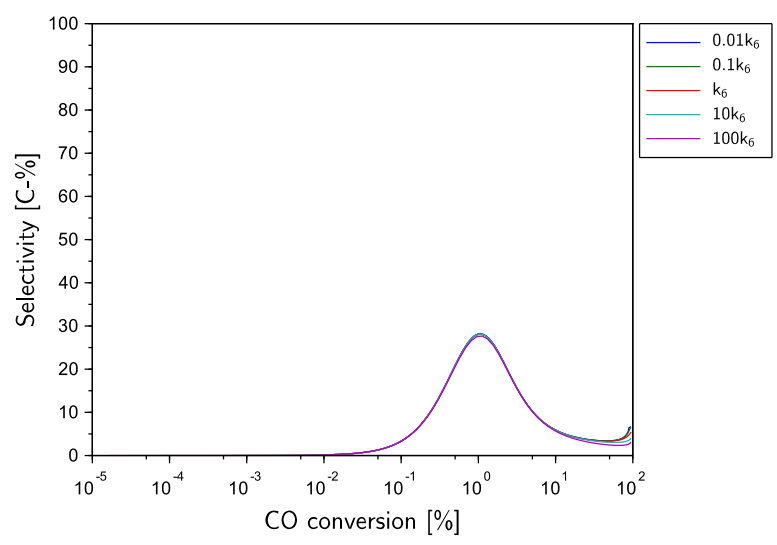

(b) Selectivity to $\mathrm{C}_{3}$

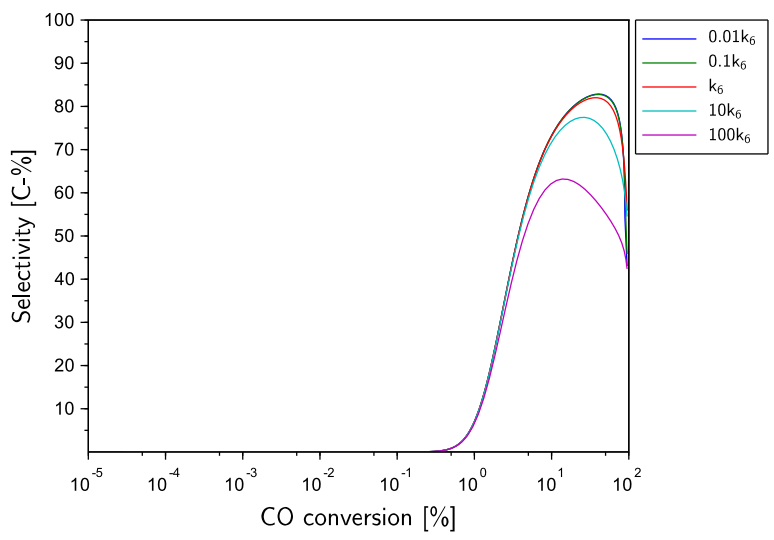

(d) Selectivity to $\mathrm{C}_{5+}$

Figure 5.10: The effect of variations of $k_{6}$ on the carbon-based selectivity at $220^{\circ} \mathrm{C}, 20 \operatorname{bar}(\mathrm{a})$ and $\mathrm{H}_{2} / \mathrm{CO}$ feed ratio of 2.1 continued

\subsection{Derivative-based Analysis}

The relative sensitivity of the product selectivity, henceforth referred to as the sensitivity function, of $\mathrm{C}_{1}, \mathrm{C}_{2}, \mathrm{C}_{3}, \mathrm{C}_{4}, \mathrm{C}_{5+}$ and $\mathrm{CO}_{2}$ with respect to each $\mathrm{k}_{\mathrm{j}}$ can be calculated using the first order finite difference approximation ${ }^{2}$ in Equation 5.6 .

$$
\frac{\partial \ln \left(S_{i}\right)}{\partial \ln \left(k_{j}\right)} \approx \frac{S_{i}\left(k_{j}+\Delta k_{j}\right)-S_{i}\left(k_{j}\right)}{\Delta k_{j}} \frac{k_{j}}{S_{i}\left(k_{j}\right)}
$$

Each rate constant is varied one at a time while the value of the other rate constants are held fixed at their base case values in Table 5.1. Thus, a total of seven model evaluations are required.

\footnotetext{
${ }^{2}$ The accuracy of the first order finite difference approximation and results are checked using the second order finite difference approximation in Appendix B.
} 
Similarly to the one-at-a-time analysis, the sensitivity functions of $\mathrm{C}_{1}, \mathrm{C}_{2}, \mathrm{C}_{3}, \mathrm{C}_{4}, \mathrm{C}_{5+}$ and $\mathrm{CO}_{2}$ with respect to each $k_{j}$ are then studied up to a CO conversion of $95 \%$ (Tucker \& van Steen, 2019).

The value of $\Delta k_{j}$ found to be most stable over the entire $\mathrm{CO}$ conversion range is given in Equation 5.7. The same step size is used in the degree of rate control analysis of Allie \& Nyathi (2019).

$$
\Delta k_{j}=0.01 k_{j}
$$

Equation 5.6 is used to calculate the sensitivity function of $\mathrm{C}_{1}, \mathrm{C}_{2}, \mathrm{C}_{3}, \mathrm{C}_{4}$ and $\mathrm{CO}_{2}$. Since the selectivity to $C_{5+}$ depends on the latter it can be calculated using Equation 5.8. These analyses are also carried out at a temperature of $220^{\circ} \mathrm{C}$, total pressure of $20 \operatorname{bar}(a)$ and $\mathrm{H}_{2} / \mathrm{CO}$ feed ratio of 2.1 .

$$
\frac{\partial \ln \left(S_{C_{5+}}\right)}{\partial \ln \left(k_{j}\right)}=\frac{k_{j}}{S_{C_{5+}}\left(k_{j}\right)}\left(-\frac{\partial S_{C_{1}}}{\partial k_{j}}-\frac{\partial S_{C_{2}}}{\partial k_{j}}-\frac{\partial S_{C_{3}}}{\partial k_{j}}-\frac{\partial S_{C_{4}}}{\partial k_{j}}-\frac{\partial S_{C O_{2}}}{\partial k_{j}}\right)
$$

\subsubsection{Sensitivity function of $C_{1}$ with respect to $k_{j}$}

The sensitivity function for $C_{1}$ with respect to $k_{1}$ is negative throughout the simulated $C O$ conversion range in Figure 5.11. A small increase in $\mathrm{k}_{1}$ favours the hydrogenation of $\mathrm{CO}$, leading to an increased formation of monomer $\mathrm{CH}_{2}$. Consequently, there is initially a rise in the selectivity to $\mathrm{C}_{1}$ due to the hydrogenation of $\mathrm{CH}_{2}$ to methane up to a $\mathrm{CO}$ conversion of $5 \%$. The increased methane and $\mathrm{CH}_{2}$ availability, however, favours n-paraffin formation of higher carbon number by favouring the forward $n$-paraffin chain growth reaction. This leads to the decrease in the sensitivity function for $\mathrm{C}_{1}$ for $\mathrm{CO}$ conversions greater than $5 \%$ until around $75 \%$. For $\mathrm{CO}$ conversions greater than $75 \%$, there is an increase in the sensitivity function for $C_{1}$ with respect to $k_{1}$, corresponding to an increase in $C_{1}$ selectivity as the reaction system approaches complete chemical equilibrium. At this point, this sensitivity function is expected to approach zero because reaction kinetics no longer determine the system behaviour.

In Figure 5.11, the sensitivity function for $C_{1}$ with respect to $k_{2}$ is positive throughout and shows the opposite trend to the sensitivity function of $C_{1}$ with respect to $k_{1}$. This is since $k_{2}$ controls the rate at which $\mathrm{CH}_{2}$ is hydrogenated to methane. Initially, the sensitivity function for $\mathrm{C}_{1}$ with respect to $k_{2}$ decreases. This is since an increase in the formation of methane favours its reaction in the forward n-paraffin chain growth reaction. This decrease occurs until a CO conversion of around $5 \%$. Thereafter, the reverse $n$-paraffin reaction is favoured leading to the increase in sensitivity function for $C_{1}$ with respect to $k_{2}$. This occurs because increased methane formation and $\mathrm{n}$-paraffin chain growth depletes $\mathrm{CH}_{2}$. The reverse $\mathrm{n}$-paraffin chain growth reaction is then favoured, leading to the formation of $\mathrm{CH}_{2}$. This occurs until a $\mathrm{CO}$ conversion of $75 \%$. Thereafter, 


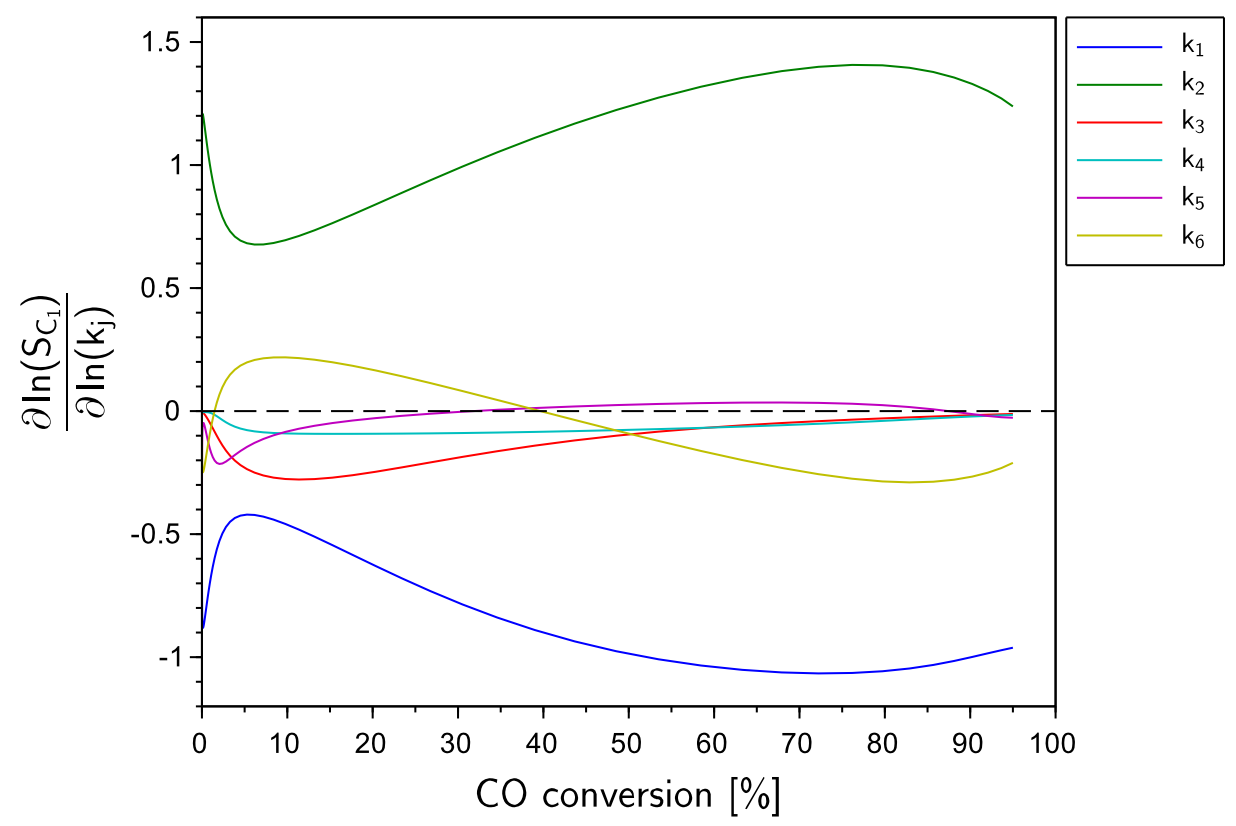

Figure 5.11: Sensitivity of $C_{1}$ with respect to each $k_{j}$

the sensitivity function for $C_{1}$ with respect to $k_{2}$ decreases as the reaction system approaches complete chemical equilibrium. Again, at complete chemical equilibrium this sensitivity function is expected to approach zero.

$C_{1}$ selectivity is most sensitive to $k_{1}$ and $k_{2}$. From Figure 5.11 the selectivity of $C_{1}$ is more sensitive to $k_{3}, k_{4}$ and $k_{5}$ for CO conversions below $5 \%$. For higher CO conversions, the selectivity to $C_{1}$ is virtually insensitive to $k_{4}$. This is because $k_{4}$ only influences the reactivity of the $n$-paraffins of carbon number $C_{2}$ and greater. The sensitivity functions for $C_{1}$ with respect to $k_{3}$ and $k_{5}$ are both negative until a $\mathrm{CO}$ conversion of about $10 \%$. The sensitivity function of $C_{1}$ with respect to $k_{3}$ determines the reactivity of methane towards ethane and hence its participation in the n-paraffin chain growth reactions. This decreases the methane selectivity and hence leads to the decrease in the sensitivity function of $C_{1}$ with respect to $k_{3}$. However, as the reverse $n$-paraffin chain growth reactions become favoured, the sensitivity function of $C_{1}$ with respect to $k_{3}$ increases, eventually becoming zero when the reaction system approaches complete chemical equilibrium. The sensitivity function of $C_{1}$ with respect to $k_{5}$ is initially negative because of the consumption of $\mathrm{CH}_{2}$ towards 1-olefins, reducing its availability for hydrogenation towards methane. However, the sensitivity function of $C_{1}$ with respect to $k_{5}$ then increases and becomes positive with increasing $C O$ conversion. This arises because of the reverse 1-olefin chain growth reaction being favoured due to the depletion of $\mathrm{CH}_{2}$ as the 1-olefin chain growth reactions approach equilibrium. Consequently, 
this increases the availability of $\mathrm{CH}_{2}$ for hydrogenation leading to the rise in methane selectivity. The sensitivity function of $C_{1}$ with respect to $k_{5}$ then decreases to zero as the reaction system approaches complete chemical equilibrium.

The behaviour of the sensitivity function of $C_{1}$ with respect to $k_{1}$ and the sensitivity function of $C_{1}$ with respect to $k_{6}$ follow the same trend. Initially, the sensitivity function of $C_{1}$ with respect to $k_{6}$ rises until a $\mathrm{CO}$ conversion of $5 \%$. This is the result of an increase in $\mathrm{H}_{2}$ formation, favouring $\mathrm{CH}_{2}$ hydrogenation towards methane. The increase in methane content, however, favours its reaction in the n-paraffin chain growth reactions. This leads to the decrease in the sensitivity function of $C_{1}$ with respect to $k_{6}$ until a $\mathrm{CO}$ conversion of $80 \%$. Thereafter, the sensitivity function of $C_{1}$ with respect to $k_{6}$ increases and approaches zero as the reaction system approaches complete chemical equilibrium.

\subsubsection{Sensitivity function of $C_{2}$ with respect to $k_{j}$}

In Figure 5.12, the sensitivity function of $C_{2}$ with respect to $k_{1}$ and $k_{2}$ again show the opposite trends. The sensitivity function of $\mathrm{C}_{2}$ with respect to $\mathrm{k}_{1}$ initially increases up to a $\mathrm{CO}$ conversion of around $3 \%$. This is since the hydrogenation of $\mathrm{CO}$ favours the formation of $\mathrm{CH}_{2}$, which favours chain growth and the formation of $\mathrm{C}_{2}$ hydrocarbons (ethane and ethene). In contrast, the sensitivity function of $\mathrm{C}_{2}$ with respect to $\mathrm{k}_{2}$ decreases over the same $\mathrm{CO}$ conversion range due to the increased reactivity of methane towards ethane and the consumption of $\mathrm{CH}_{2}$ towards ethene. However, with increasing $\mathrm{CO}$ conversion, the behaviour of these two sensitivity functions reverse. The sensitivity function of $C_{2}$ with respect to $k_{1}$ decreases as ethane and ethene react to form n-paraffins and 1olefins of higher carbon number. Between a CO conversion of 30\% to $70 \%$ the sensitivity function of $C_{2}$ with respect to $k_{1}$ is constant indicating no change in $C_{2}$ selectivity. The sensitivity function of $\mathrm{C}_{2}$ with respect to $\mathrm{k}_{2}$, however, continues to increase until a $\mathrm{CO}$ conversion of around $70 \%$. This indicates that chain growth beyond $\mathrm{C}_{2}$ is limited due to the preferential hydrogenation of $\mathrm{CH}_{2}$ towards methane. Both of these sensitivity functions decrease and approach zero as the reaction system approaches complete chemical equilibrium.

The sensitivity functions for $C_{2}$ with respect to $k_{3}, k_{4}$ and $k_{5}$ in Figure 5.12 are all initially negative. The sensitivity function for $C_{2}$ with respect to $k_{3}$ initially decreases until a $C O$ conversion of $3 \%$. This arises because of the increased reactivity towards ethane, favouring further chain growth to higher carbon number n-paraffins. Thereafter, with increases in $\mathrm{CO}$ conversion, the sensitivity functions for $C_{2}$ with respect to $k_{3}$ increases until a $C O$ conversion of $15 \%$ and becomes positive. This indicates that the $\mathrm{C}_{2}$ selectivity increases and that the reverse n-paraffin chain growth reaction is favoured. Thereafter, the sensitivity function for $C_{2}$ with respect to $k_{3}$ decreases as the $C O$ conversion increases and approaches zero at complete chemical equilibrium. The sensitivity func- 


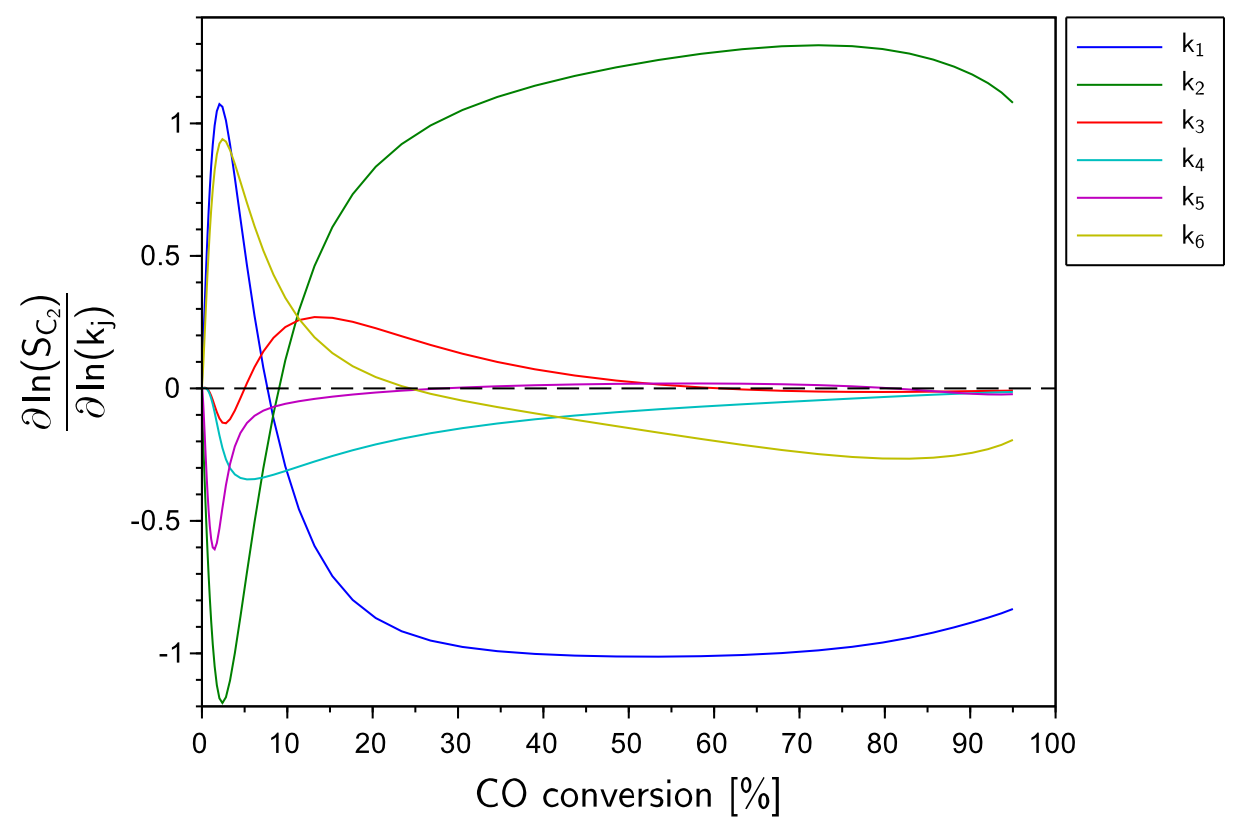

Figure 5.12: Sensitivity of $C_{2}$ with respect to each $\mathrm{k}_{\mathrm{j}}$

tion for $\mathrm{C}_{2}$ with respect to $\mathrm{k}_{4}$ remains negative across the entire $\mathrm{CO}$ conversion range. Initially, the sensitivity function for $C_{2}$ with respect to $k_{4}$ decreases until a $C O$ conversion of $5 \%$. This arises because $\mathrm{k}_{4}$ favours the reaction of ethane towards higher carbon number $n$-paraffins. This corresponds to a decrease in $\mathrm{C}_{2}$ selectivity. As the $\mathrm{CO}$ conversion increases, the sensitivity function for $C_{2}$ with respect to $k_{4}$ increases. This arises because the reverse $n$-paraffin chain growth reaction becomes favoured. Eventually, the sensitivity function for $\mathrm{C}_{2}$ with respect to $\mathrm{k}_{4}$ will approach zero as the reaction system attains complete chemical equilibrium. The sensitivity function for $C_{2}$ with respect to $k_{5}$ follows the same behaviour.

The sensitivity function for $C_{2}$ with respect to $k_{6}$ follows the same behaviour as the sensitivity function for $C_{2}$ with respect to $k_{1}$. Initially, the sensitivity function for $C_{2}$ with respect to $k_{6}$ increases up to a $\mathrm{CO}$ conversion of around $3 \%$. This corresponds to an increase in $\mathrm{C}_{2}$ selectivity due to an increase in $\mathrm{H}_{2}$ formation, favouring $\mathrm{CH}_{2}$ hydrogenation to methane and hence reaction towards ethane. Thereafter, the sensitivity function for $C_{2}$ with respect to $k_{6}$ decreases. This corresponds to a decrease in $\mathrm{C}_{2}$ selectivity as ethane reacts to form higher carbon number $\mathrm{n}$ paraffins. Between a CO conversion of $15-20 \%$, there is a change in concavity of the sensitivity function for $\mathrm{C}_{2}$ with respect to $\mathrm{k}_{6}$ as it continues to decrease until a $\mathrm{CO}$ conversion of $80 \%$. This is due to the competition between the CO hydrogenation and WGS reactions, limiting the formation of hydrocarbons other than methane. The sensitivity function for $C_{2}$ with respect to $k_{6}$ thereafter 
increases and approaches zero as the reactions approaches complete chemical equilibrium.

\subsubsection{Sensitivity function of $C_{3}$ with respect to $k_{j}$}

In Figure 5.13, the sensitivity functions for $C_{3}$ with respect to $k_{1}$ and $k_{2}$ again show the opposite trends, as before. Initially, the sensitivity function for $C_{3}$ with respect to $k_{1}$ is positive and increasing until a CO conversion of $3 \%$. This can be explained in the same way as for the sensitivity function for sensitivity for $C_{2}$ with respect to $k_{1}$, arising because of favourable chain growth towards $C_{3}$ hydrocarbons. With increases in $\mathrm{CO}$ conversion, the selectivity to $C_{3}$ decreases due to chain growth towards higher hydrocarbons. In contrast, the sensitivity function for $C_{3}$ with respect to $k_{2}$ initially decreases. This corresponds to a decrease in $C_{3}$ selectivity as chain growth is limited, since a relative increase in $\mathrm{k}_{2}$ favours methane formation. With increases in $\mathrm{CO}$ conversion, the sensitivity function for $C_{3}$ with respect to $k_{2}$, increases indicating an increase in $C_{3}$ selectivity. This arises because of increased methane reactivity towards higher carbon n-paraffins. Both the sensitivity function for $C_{3}$ with respect to $k_{1}$ and $k_{2}$ will approach zero as the reaction approaches complete chemical equilibrium.

The sensitivity functions for $\mathrm{C}_{3}$ with respect to $\mathrm{k}_{3}$ initially increases, before decreasing until a $\mathrm{CO}$ conversion of $3 \%$. This occurs because of the increased reactivity of ethane towards propane, before propane then reacts further to form n-paraffins of higher carbon number. Thereafter, the sensitivity function for $C_{3}$ with respect to $k_{3}$ increases indicating an increased selectivity back to $\mathrm{C}_{3}$. This occurs because the reverse $n$-paraffin chain growth reaction is favoured, favouring the formation of $\mathrm{CH}_{2}$. The sensitivity function for $\mathrm{C}_{3}$ with respect to $\mathrm{k}_{3}$ then decreases and approaches zero as the reaction approaches complete chemical equilibrium, where methane is the dominant hydrocarbon product. The sensitivity function for $C_{3}$ with respect to $k_{4}$ follows the same behaviour.

From Figure 5.13, the selectivity to $C_{3}$ is initially most sensitive to $k_{5}$ since propene is the dominant $C_{3}$ hydrocarbon. The sensitivity function for $C_{3}$ with respect to $k_{5}$ then decreases as propene reacts further in the 1-olefin chain growth reactions. This occurs until a $\mathrm{CO}$ conversion of $3 \%$. Thereafter, the sensitivity function for $C_{3}$ with respect to $k_{5}$ increases as the reverse 1-olefin chain growth reaction is favoured. Between a CO conversion of $20 \%$ and $80 \%$, the sensitivity function for $C_{3}$ with respect to $k_{5}$ is constant, indicating no change in $C_{3}$ selectivity. The sensitivity function for $C_{3}$ with respect to $k_{5}$ then decreases and approaches zero as the reactions approach complete chemical equilibrium.

The sensitivity function for $C_{3}$ with respect to $k_{6}$ follows the same behaviour as the sensitivity function for $C_{3}$ with respect to $k_{1}$. There is initially a rise in the sensitivity function for $C_{3}$ with 


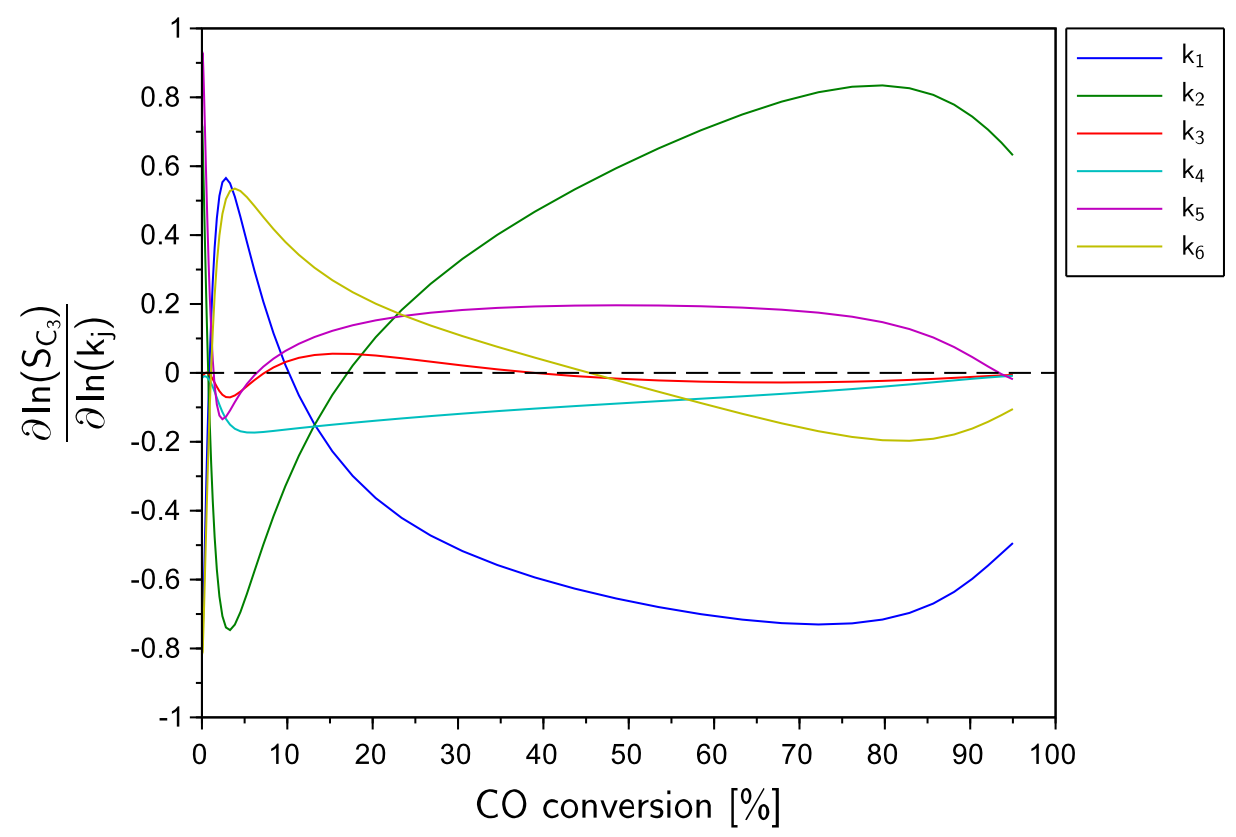

Figure 5.13: Sensitivity of $C_{3}$ with respect to each $\mathrm{k}_{\mathrm{j}}$

respect to $\mathrm{k}_{6}$ until a $\mathrm{CO}$ conversion of $5 \%$. This arise because of an increase in $\mathrm{H}_{2}$ formation favouring $\mathrm{CH}_{2}$ hydrogenation and hence chain growth towards higher carbon number n-paraffins. Thereafter, the sensitivity function for $C_{3}$ with respect to $k_{6}$ decreases, indicating a decrease in $C_{3}$ selectivity. This occurs due to CO reacting in the WGS reaction rather than being hydrogenated. The sensitivity function for $C_{3}$ with respect to $k_{6}$ then increases and approaches zero as the reaction approaches complete chemical equilibrium.

\subsubsection{Sensitivity function of $\mathrm{C}_{4}$ with respect to $\mathrm{k}_{\mathrm{j}}$}

In Figure 5.14, the sensitivity function for $C_{4}$ with respect to $k_{1}$ and $k_{2}$ respond in the same way as the sensitivity functions for $C_{2}$ and $C_{3}$, also with respect to $k_{1}$ and $k_{2}$. This is because an increase in $\mathrm{k}_{1}$ favours the formation of $\mathrm{CH}_{2}$. The initial rise in $\mathrm{CH}_{2}$ formation favours further chain growth leading to the increase in the sensitivity function for $\mathrm{C}_{4}$ with respect to $\mathrm{k}_{1}$ up to a $\mathrm{CO}$ conversion of $3 \%$. The decrease in the sensitivity function for $C_{4}$ with respect to $k_{1}$ with increases in $C O$ conversion occurs because of the decrease in $C_{4}$ selectivity as $C_{4}$ hydrocarbons react further to hydrocarbons of higher carbon number. The sensitivity function for $C_{4}$ with respect to $k_{1}$, however, shows a change in concavity. This can be interpreted as the reverse chain growth reactions being favoured, as the reaction system approaches complete chemical equilibrium.

The sensitivity function for $\mathrm{C}_{4}$ with respect to $\mathrm{k}_{2}$ decreases up to a $\mathrm{CO}$ conversion of $3 \%$. This is 


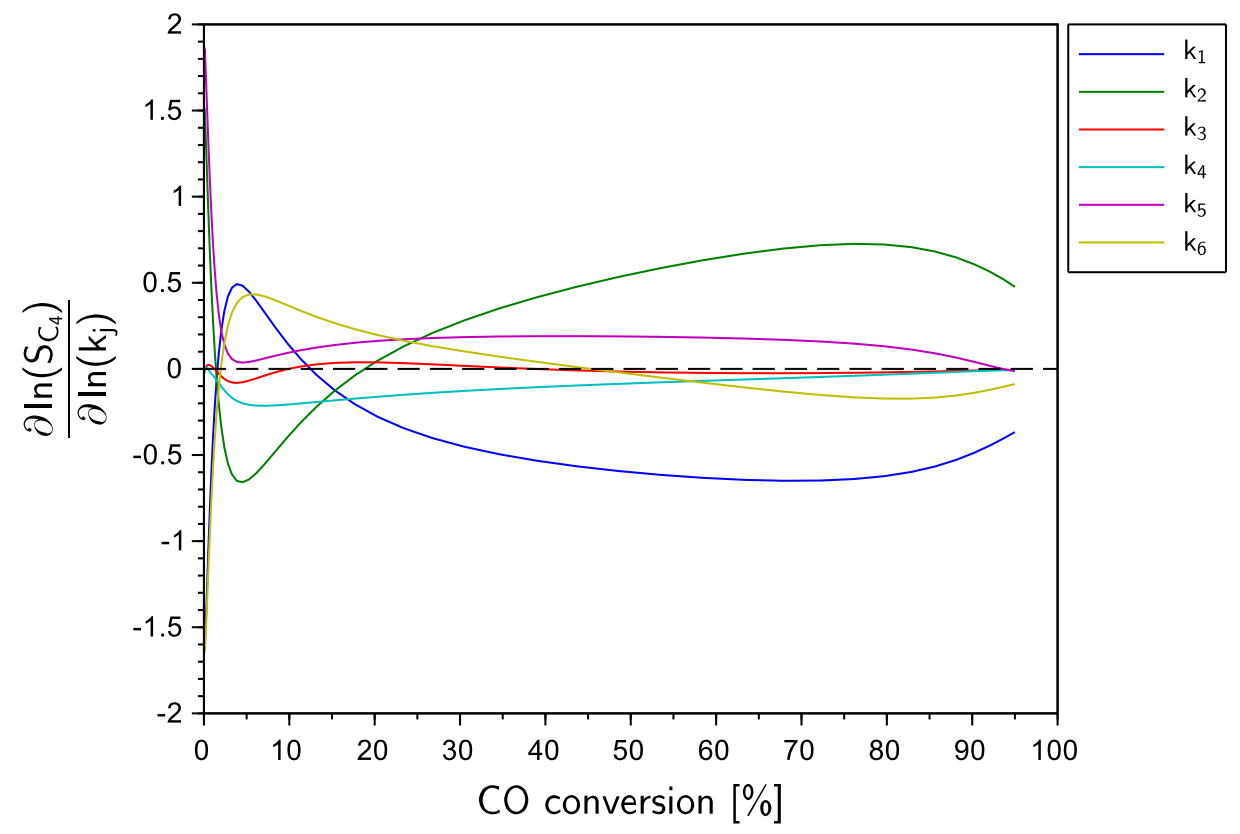

Figure 5.14: Sensitivity of $C_{4}$ with respect to each $\mathrm{k}_{\mathrm{j}}$

the result of $\mathrm{CH}_{2}$ being preferentially hydrogenated and reacting to form methane. The sensitivity function for $C_{4}$ with respect to $k_{2}$ then increases and becomes positive. This is the result of the increase in methane formation, favouring its reaction in the n-paraffin chain growth reactions, leading to a rise in $C_{4}$ selectivity. The sensitivity function for $C_{4}$ with respect to $k_{2}$ also changes concavity. This can be interpreted as the reverse chain growth reactions being favoured, as these reactions approach their equilibrium position. It is expected that both the sensitivity function for $\mathrm{C}_{4}$ with respect to $k_{1}$ and $k_{2}$ will approach zero as the reaction system approaches complete chemical equilibrium.

From Figure 5.14, the selectivity to $C_{4}$ is more sensitive to $k_{4}$ and most sensitive to $k_{5}$ relative to $k_{3}$. The low sensitivity of the $C_{4}$ selectivity to $k_{3}$ arises because it determines the rate at which methane reacts to ethane. This initially leads to a rise in $C_{4}$ selectivity through favourable chain growth to $n$-butane, shown by the increase in the sensitivity function for $C_{4}$ with respect to $k_{3}$. This, however, then favours further reaction to n-paraffins of higher carbon number, leading to a decrease in the sensitivity function for $C_{4}$ with respect to $k_{3}$. With further increases in $C O$ conversion, the sensitivity function for $C_{4}$ with respect to $k_{3}$ increases. This arises because of the increase in $C_{4}$ selectivity as the reverse $n$-paraffin chain growth reaction is favoured. The sensitivity function for $C_{4}$ with respect to $k_{3}$ then decreases as selectivity shifts away from $C_{4}$ to lower carbon numbers as the reaction system approaches complete chemical equilibrium. 
The sensitivity function for $C_{4}$ with respect to $k_{4}$ is negative throughout, and initially decreasing, as $k_{4}$ favours the reaction of $n$-butane to higher carbon number $n$-paraffins. This occurs until a CO conversion of $3 \%$. Thereafter, the sensitivity function for $C_{4}$ with respect to $\mathrm{k}_{4}$ increases as the reverse n-paraffin chain growth reaction is favoured, shifting selectivity back towards lower carbon number $\mathrm{n}$-paraffins. The sensitivity function for $\mathrm{C}_{4}$ with respect to $\mathrm{k}_{4}$ approaches zero as the reaction system approaches complete chemical equilibrium, where selectivity shifts to methane.

The selectivity to $C_{4}$ is initially most sensitive to $k_{5}$ since 1 -butene accounts for the majority of the $\mathrm{C}_{4}$ selectivity. The sensitivity function for $\mathrm{C}_{4}$ with respect to $\mathrm{k}_{5}$ decreases until a $\mathrm{CO}$ conversion of $3 \%$. This arises because of further chain growth to higher carbon number 1-olefins. Thereafter, the sensitivity function for $\mathrm{C}_{4}$ with respect to $\mathrm{k}_{5}$ increases until a $\mathrm{CO}$ conversion of $20 \%$. This indicates that the reverse 1-olefin chain growth reaction becomes favoured, as these reactions approach equilibrium. Between a CO conversion of $20 \%$ and $80 \%$, the sensitivity function for $\mathrm{C}_{4}$ with respect to $k_{5}$ is constant, indicating no change in selectivity to $C_{4}$. Thereafter, the sensitivity function for $C_{4}$ with respect to $k_{5}$ decreases as the reaction system approaches complete chemical equilibrium.

In Figure 5.14, the sensitivity function for $C_{4}$ with respect to $k_{6}$ responds similarly to the sensitivity function for $C_{4}$ with respect to $k_{1}$. Initially, the sensitivity function for $C_{4}$ with respect to $k_{6}$ increases, indicating an increase in $\mathrm{C}_{4}$ selectivity. This occurs because of an increase in $\mathrm{H}_{2}$ formation, favouring $\mathrm{CH}_{2}$ hydrogenation and hence n-paraffin chain growth. Thereafter, with increases in $\mathrm{CO}$ conversion, the sensitivity function for $\mathrm{C}_{4}$ with respect to $\mathrm{k}_{6}$ decreases. This indicates a decrease in $\mathrm{C}_{4}$ selectivity as $\mathrm{CO}$ preferentially reacts in the WGS reaction rather than being hydrogenated. The sensitivity function for $C_{4}$ with respect to $k_{6}$ then increases and approaches zero as the reaction system approaches complete chemical equilibrium.

\subsubsection{Sensitivity function of $C_{5+}$ with respect to $k_{j}$}

In Figure 5.15, the sensitivity function for $C_{5+}$ with respect to $k_{1}$ and $k_{2}$ once again show the same behaviour as before, with equal but opposite trends. That is, a relative increase in $k_{1}$ favours the formation of and selectivity to $\mathrm{C}_{5+}$ hydrocarbons, while a relative increase in $\mathrm{k}_{2}$ limits the formation and selectivity to $\mathrm{C}_{5+}$. This is expected since $\mathrm{k}_{1}$ produces $\mathrm{CH}_{2}$, favouring chain growth while $\mathrm{k}_{2}$ leads to the hydrogenation of $\mathrm{CH}_{2}$ to methane which limits chain growth. The selectivity to $\mathrm{C}_{5+}$ is most responsive to $\mathrm{k}_{3}, \mathrm{k}_{4}$ and $\mathrm{k}_{5}$ for $\mathrm{CO}$ conversions up to $5 \%$. This indicates that the selectivity to $\mathrm{C}_{5+}$ hydrocarbons is established at low $\mathrm{CO}$ conversions due to the fast nature of the chain growth reactions, relative to the initial hydrogenation of $\mathrm{CO}$. For $\mathrm{CO}$ conversions greater than $5 \%$, the sensitivity function for $C_{5+}$ with respect to $k_{3}, k_{4}$ and $k_{5}$ are all zero. This indicates that at higher $\mathrm{CO}$ conversions, the reversibility of the n-paraffin and 1-olefin chain growth reactions 
determines the $\mathrm{C}_{5+}$ selectivity. This reinforces that the $\mathrm{C}_{5+}$ is thermodynamically controlled for higher $\mathrm{CO}$ conversions as the reaction system approaches complete chemical equilibrium. Again, the sensitivity function for $C_{5+}$ with respect to $k_{6}$ follows the same trend as the sensitivity function for $\mathrm{C}_{5+}$ with respect to $\mathrm{k}_{1}$.

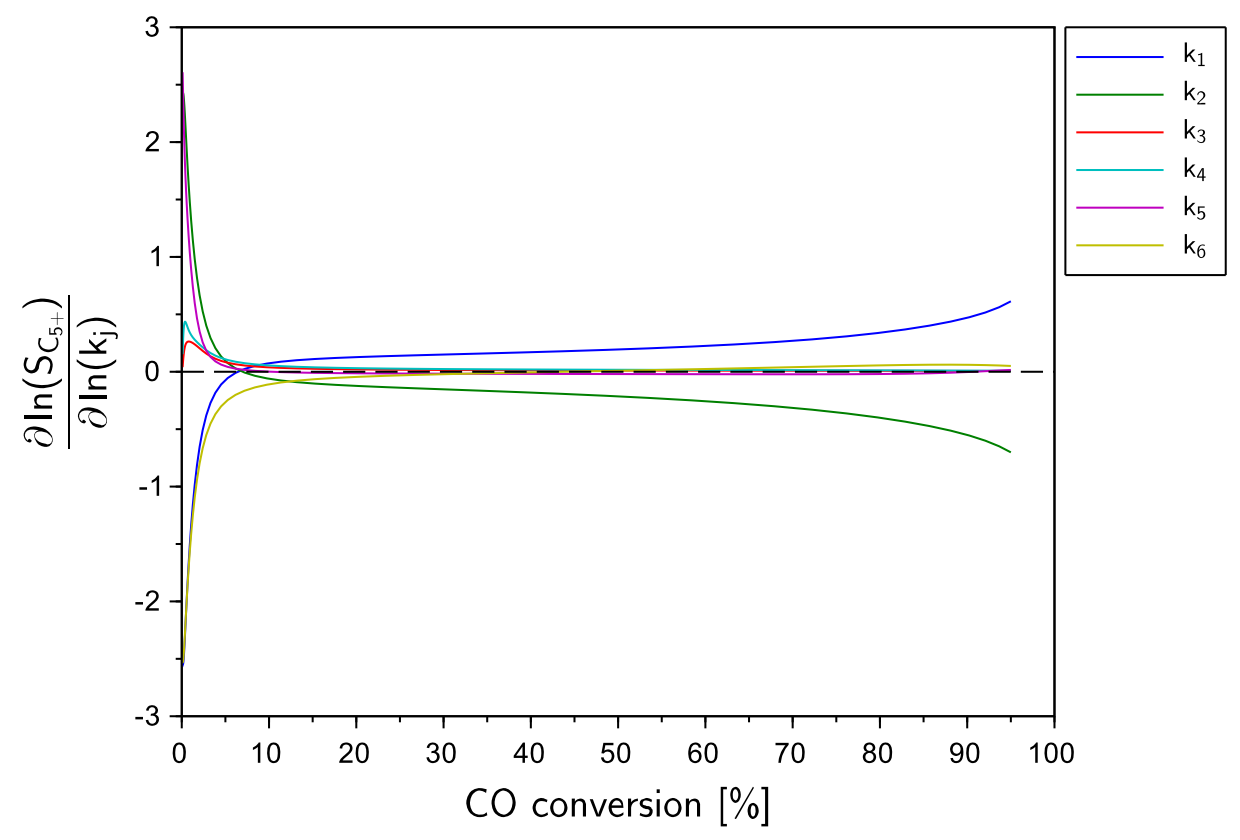

Figure 5.15: Sensitivity of $C_{5+}$ with respect to each $k_{j}$

\subsubsection{Sensitivity function of $\mathrm{CO}_{2}$ with respect to $\mathrm{k}_{\mathrm{j}}$}

In Figure 5.16, the selectivity to $\mathrm{CO}_{2}$ is insensitive to $\mathrm{k}_{3}, \mathrm{k}_{4}$ and $\mathrm{k}_{5}$. This is since these rate constants influence hydrocarbon reactivity and chain growth. The most important rate constants which drive the selectivity to $\mathrm{CO}_{2}$ are $\mathrm{k}_{1}$ and $\mathrm{k}_{2}$ rather than $\mathrm{k}_{6}$, despite its function as the WGS rate constant. From Figure 5.16, $\mathrm{CO}_{2}$ selectivity is most sensitive to $\mathrm{k}_{1}$.

The rate constants $\mathrm{k}_{1}$ and $\mathrm{k}_{6}$ determine the reactivity of $\mathrm{CO}$ along two different reaction paths. The WGS reaction, however, cannot occur until water forms. Both $k_{1}$ and $k_{6}$ have the same numerical value in this analysis, but due to the equilibrium constant $\mathrm{K}_{\mathrm{a}, 1}$ being almost two orders of magnitude larger than $\mathrm{K}_{\mathrm{a}, 2 \mathrm{~N}+1}$, the WGS reaction is then also limited by the preferential consumption of $\mathrm{CO}$ through hydrogenation (see Appendix A.3). The sensitivity function for $\mathrm{CO}_{2}$ with respect to $\mathrm{k}_{1}$ is therefore negative, since $\mathrm{k}_{1}$ limits the selectivity to $\mathrm{CO}_{2}$. The rise in the sensitivity function for $\mathrm{CO}_{2}$ with respect to $\mathrm{k}_{1}$ is, however, the consequence of water formation leading to an increase in $\mathrm{CO}_{2}$ selectivity. 


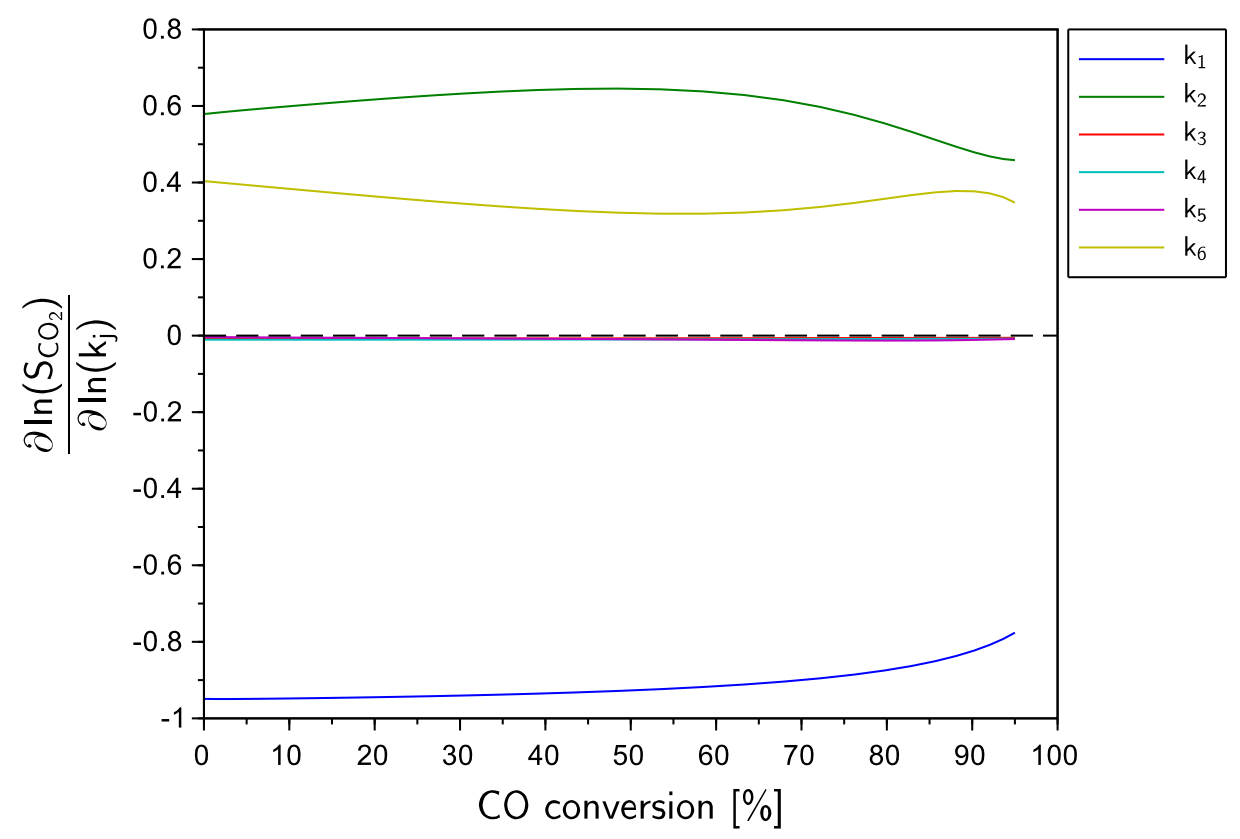

Figure 5.16: Sensitivity of $\mathrm{CO}_{2}$ with respect to each $\mathrm{k}_{\mathrm{j}}$

Similarly, the selectivity to $\mathrm{CO}_{2}$ is much more responsive to $\mathrm{k}_{2}$. This is since $\mathrm{k}_{2}$ results in the hydrogenation of $\mathrm{CH}_{2}$. The consumption of $\mathrm{H}_{2}$ then drives the forward WGS reaction leading to an increase in $\mathrm{CO}_{2}$ selectivity. This, however, increases $\mathrm{H}_{2}$ formation which favours $\mathrm{CH}_{2}$ hydrogenation and hence the formation of and selectivity to methane. Consequently, the sensitivity function for $\mathrm{CO}_{2}$ with respect to $\mathrm{k}_{6}$ decreases as the reaction system approaches complete chemical equilibrium.

It is clear from Figure 5.16, that $\mathrm{CO}_{2}$ selectivity is more responsive to $\mathrm{k}_{1}$ and $\mathrm{k}_{2}$ because of their influence on the formation of water, in the case of $\mathrm{k}_{1}$, and the consumption of $\mathrm{H}_{2}$ in the case of both $\mathrm{k}_{1}$ and $\mathrm{k}_{2}$. Both water and $\mathrm{H}_{2}$ are reactants in the WGS reaction, whose formation (water) and consumption $\left(\mathrm{H}_{2}\right)$ drive the forward WGS reaction and thus determine the selectivity to $\mathrm{CO}_{2}$. This is consistent with the findings in Figure 5.16, where the effect of $k_{6}$ is less pronounced in comparison to $\mathrm{k}_{1}$ and $\mathrm{k}_{2}$ in determining the $\mathrm{CO}_{2}$ selectivity.

\subsubsection{Key Features}

The results of the sensitivity analysis indicate the importance of the rate constant $k_{1}$. This is shown by its influence and control on the selectivity to all products. This highlights the importance of CO hydrogenation as a rate-determining reaction step. A relative increase in $\mathrm{k}_{1}$ favours $\mathrm{CH}_{2}$ formation. This reduces the selectivity to methane and favours selectivity to higher carbon number products. Furthermore, this also determines the selectivity to $\mathrm{CO}_{2}$ through the formation of water. The rate 
constant $k_{2}$ is the methanation rate constant. A relative increase in $k_{2}$ thus favours selectivity to methane while limiting chain growth by preferentially hydrogenating $\mathrm{CH}_{2}$.

The selectivity to hydrocarbons of carbon number greater than $C_{1}$ is also more sensitive to $k_{1}$ and $k_{2}$ than $k_{3}-k_{5}$ over the entire CO conversion range. This arises because $k_{1}$ and $k_{2}$ determines the reactivity of $\mathrm{CH}_{2}$ along two competing reaction pathways, namely methanation and chain growth. Furthermore, the rate constants $k_{3}-k_{5}$ only influence the selectivity of $C_{2}-C_{5+}$ for low $C O$ conversions. This is consistent with the relative rates of chain growth being much greater than $\mathrm{CO}$ hydrogenation. This leads to the insensitivity of the selectivity, particularly for $\mathrm{C}_{5+}$, to the rate constants $k_{3}-k_{5}$ for higher $\mathrm{CO}$ conversions.

Up to now, it has been assumed that all the rate constant $k_{1}-k_{6}$ are independent of one another. The dependence between the rate constants can be assessed by evaluating the ratio of the sensitivity functions to reveal over-parametrisation of the model. This will be essential in providing insight into which parameters can be determined by regression.

Throughout the sensitivity analysis, the sensitivity functions with respect to $k_{1}$ and $k_{6}$ follow the same pattern. Both the rate constants $\mathrm{k}_{1}$ and $\mathrm{k}_{6}$ influence the reactivity of $\mathrm{CO}$, by determining whether it reacts along the hydrogenation or WGS reaction pathways. However, it was shown that the selectivity to $\mathrm{CO}_{2}$ depends more strongly on $\mathrm{k}_{1}$ and $\mathrm{k}_{2}$ than $\mathrm{k}_{6}$. The rate constant $\mathrm{k}_{1}$ thus dominates the influence of the rate constant $k_{6}$ on the WGS reactivity, which reinforces its rate control in the reactivity of $\mathrm{CO}$. The sensitivity of the $\mathrm{CO}_{2}$ selectivity to $\mathrm{k}_{2}$ means that it can be used as common basis to determine the dependence between $\mathrm{k}_{1}$ and $\mathrm{k}_{6}$.

In Figure 5.17a, the relationship for the ratios of the sensitivity functions for $\mathrm{CO}_{2}$ selectivity is shown. As can be seen and quantified by the $R^{2}$ value in Table 5.2, the degree of linear correlation is excellent, confirming the linear dependence between the ratios of the sensitivity functions for $\mathrm{CO}_{2}$ selectivity. This indicates that the linear relationship between $\mathrm{k}_{1}, \mathrm{k}_{2}$ and $\mathrm{k}_{6}$ will be regressed rather than their actual values when using product selectivity as a function of $\mathrm{CO}$ conversion data.

Table 5.2: Regression constants in Equation 5.5 from the linear dependence of the ratio of sensitivity functions

\begin{tabular}{|c|c|c|c|}
\hline Relationship & $a_{i}$ & $\mathrm{~b}_{\mathrm{i}}$ & $\mathrm{R}^{2}$ \\
\hline Figure $5.17 a$ & $-0.9450 \pm 0.0077$ & $-1.002 \pm 0.005$ & 0.9978 \\
\hline Figure $5.17 \mathrm{~b}$ & - & $0.9948 \pm 0.0014$ & 0.9998 \\
\hline Figure $5.17 \mathrm{c}$ & - & $-2.011 \pm 0.001$ & 0.9999 \\
\hline Figure $\overline{5.17 \mathrm{~d}}$ & $0.04728 \pm 0.00322$ & $1.348 \pm 0.002$ & 0.9998 \\
\hline
\end{tabular}

In Figure 5.17b, the relationship between $k_{1}, k_{3}$ and $k_{5}$ is evaluated through their effect on the 


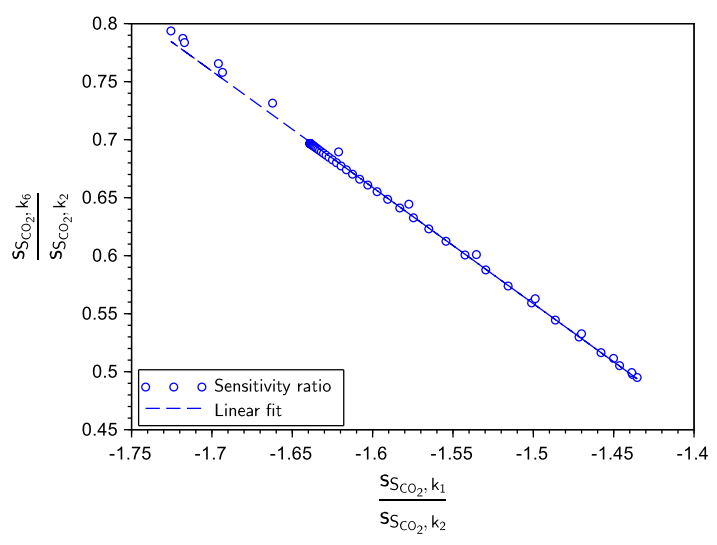

(a) Relationship between $k_{1}, k_{2}$ and $k_{6}$

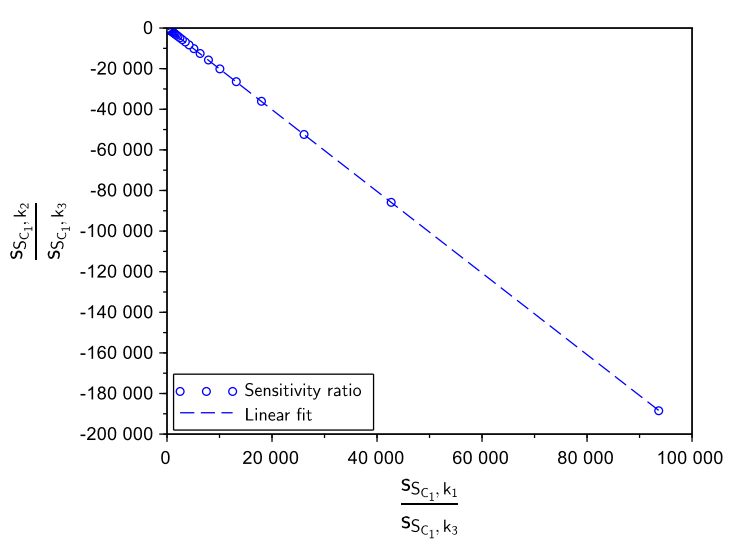

(c) Relationship between $\mathrm{k}_{1}, \mathrm{k}_{2}$ and $\mathrm{k}_{3}$

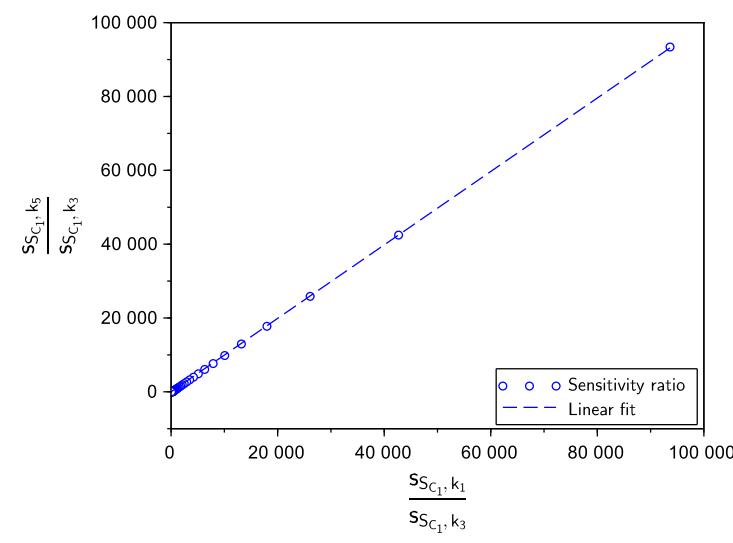

(b) Relationship between $\mathrm{k}_{1}, \mathrm{k}_{3}$ and $\mathrm{k}_{5}$

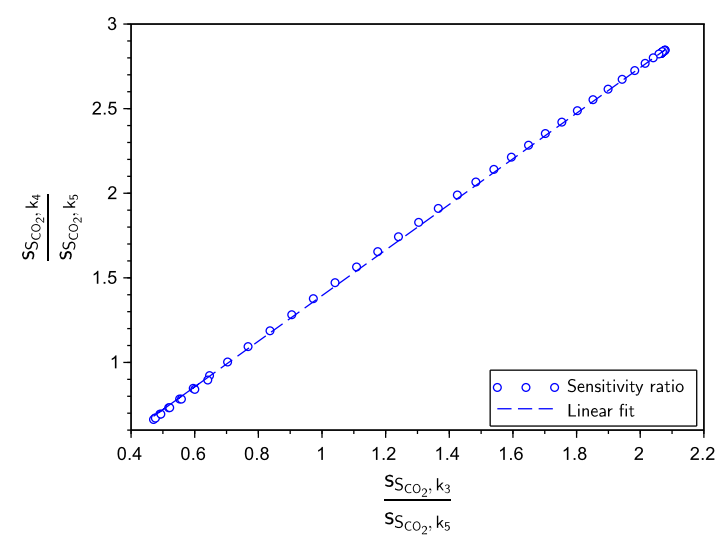

(d) Relationship between $k_{3}, k_{4}$ and $k_{5}$

Figure 5.17: Linear dependence between the ratios of the sensitivity functions with respect to the rate constants 
selectivity to $C_{1}$. The parameters $k_{1}, k_{3}$ and $k_{5}$ are correlated because of the good fit of the linear relationship to the ratio of sensitivity functions. This is confirmed by the $R^{2}$ value of 0.9999 in Table 5.2. This arises because the rate constants $k_{1}, k_{3}$ and $k_{5}$ affects the reactivity of $\mathrm{CH}_{2}$ towards the primary products methane and ethene and their subsequent participation in further chain growth.

Similarly, Figure $5.17 \mathrm{c}$ shows that the parameters $\mathrm{k}_{1}, \mathrm{k}_{2}$ and $\mathrm{k}_{3}$ are correlated. This is quantified by the closeness of the $R^{2}$ value in Table 5.2 to one. This arises because $k_{2}$ and $k_{3}$ determines the rate of formation of methane from $\mathrm{CH}_{2}$ and its subsequent reaction with $\mathrm{CH}_{2}$ in the n-paraffin chain growth reactions. Furthermore, the rate constants $k_{3}, k_{4}$ and $k_{5}$ were also shown to be linearly dependent in Figure $5.17 \mathrm{~d}$.

Evidently, a high degree of parameter correlation exists. This indicates that if product selectivity as a function of $\mathrm{CO}$ conversion data is used to determine values of $k_{1}-k_{6}$, then a meaningful estimation of all these rate constants is not possible. This arises because it is challenging to distinguish between the effect each rate constant has on product selectivity as a function of $\mathrm{CO}$ conversion. Two strategies are, however, possible to overcome this challenge:

1. The results in Figure 5.17, can be used to develop a relationship between the rate constants. The model rate constants can be replaced by a reduced rate constant set which can be determined by regression of product selectivity as a function of CO conversion data (Schwan, 2001). Relationships describing the correlation between rate constants can be formulated using a moment analysis.

2. Instead of using product selectivity as a function of CO conversion data to determine values of the model rate constants, product distribution data at constant $\mathrm{CO}$ conversion could be used.

The viability of the second strategy, however, needs to be investigated. This involves investigating the relative sensitivity of the product distribution to the rate constants $k_{1}-k_{6}$.

\subsubsection{Product Distribution Sensitivity with respect to each $\mathbf{k}_{\mathrm{j}}$}

In literature (Visconti et al., 2007, 2011), it is common to use the total hydrocarbon product distribution at constant $\mathrm{CO}$ conversion to regress for the model rate constants. The hydrocracking work of Le Grange (2009) took a similar approach, where it was shown that model rate constants could be determined from the regression of product distribution data. However, the resulting estimates were shown to be dependent on the initial guess. This indicates that the solution is not unique. A sensitivity analysis is therefore performed to provide insight regarding which rate 
constants, $\mathrm{k}_{1}-\mathrm{k}_{6}$, can be regressed from the total hydrocarbon product distribution. At constant CO conversion, the relative sensitivity $s_{w_{n}, k_{j}}$ of the weight fraction of carbon number $n$ with respect to each rate constant $k_{j}$ is given by Equation 5.9. Equation 5.9 is used to evaluate the relative sensitivity of the product distribution to each rate constant $k_{j}$ at a CO conversion of $20 \%, 40 \%$, $60 \% 80 \%$ and $95 \%$.

$$
s_{w_{n}, k_{j}}=\frac{k_{j}}{w_{n}} \frac{\partial w_{n}}{\partial k_{j}} \approx \frac{k_{j}}{w_{n}} \frac{w_{n}\left(k_{j}+\Delta k_{j}\right)-w_{n}\left(k_{j}\right)}{\Delta k_{j}}
$$

In Figure 5.18, the sensitivity of the product distribution to each rate constant $k_{j}$ is shown. A relative increase in $k_{1}$ in Figure 5.18a, favours chain growth and as such an increase in product weight product fractions from $\mathrm{C}_{20}$ onwards at each $\mathrm{CO}$ conversion. This is since $\mathrm{k}_{1}$ determines the availability of monomer $\mathrm{CH}_{2}$ and hence the rate at which it is coupled to form hydrocarbons. This emphasises that $k_{1}$ is rate-determining. In contrast, from Figure 5.18b, at each CO conversion, a relative increase in $\mathrm{k}_{2}$ favours the formation of low carbon number products. This is since $\mathrm{CH}_{2}$ is preferentially hydrogenated to methane. This reduces the availability of $\mathrm{CH}_{2}$ for reaction through chain growth. Figure 5.18a and 5.18b indicates that the product distribution is most sensitive to $k_{1}$ and $k_{2}$ across all carbon numbers and that these two rate constants have opposite effects on the product distribution.

In Figure $5.18 \mathrm{C}$ and $5.18 \mathrm{~d}$, the product distribution is most sensitive to $\mathrm{k}_{3}$ and $\mathrm{k}_{4}$ at a CO conversion of $20 \%$. The total hydrocarbon product distribution responds to $k_{3}$ by favouring the formation of ethane in Figure $5.18 \mathrm{C}$ at a CO conversion of $20 \%$. This favours the reaction of methane and consequently favours n-paraffin chain growth, shown by the positive relative sensitivity for products of carbon number $\mathrm{C}_{15}-\mathrm{C}_{30}$. The rate constant $\mathrm{k}_{4}$ favours the reaction of $n$-paraffins of carbon number $C_{2}$ and greater to higher carbon number n-paraffins through chain growth in Figure $5.18 \mathrm{~d}$. This highlights that the rate constants $k_{3}$ and $k_{4}$ establish the nature of the product distribution at low CO conversion due to the rapid rate of chain growth. With increases in CO conversion, the product distribution becomes less sensitive to $\mathrm{k}_{3}$ and $\mathrm{k}_{4}$. This arises due to an increase in the rate of the reverse $n$-paraffin chain growth reactions. This emphasises that reaction kinetics alone do not determine the nature of the total hydrocarbon product distribution.

In Figure 5.18e, a relative increase in $k_{5}$ favours the formation of product of low carbon number. With increasing CO conversion from 20\%, the total hydrocarbon product distribution becomes less sensitive to $k_{5}$ for products of carbon number $C_{2}-C_{20}$. This is attributed to the high $n$ paraffin content of the product distribution at higher $\mathrm{CO}$ conversion. In Figure 5.18f, the total hydrocarbon product distribution is insensitive to $k_{6}$ until a CO conversion of $60 \%$. This is since the WGS reaction cannot occur until water forms from the hydrogenation of CO. 


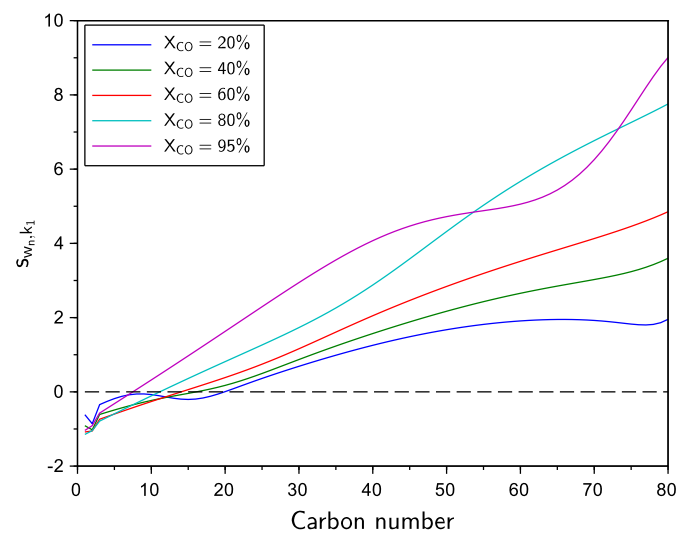

(a) Product distribution sensitivity to $k_{1}$

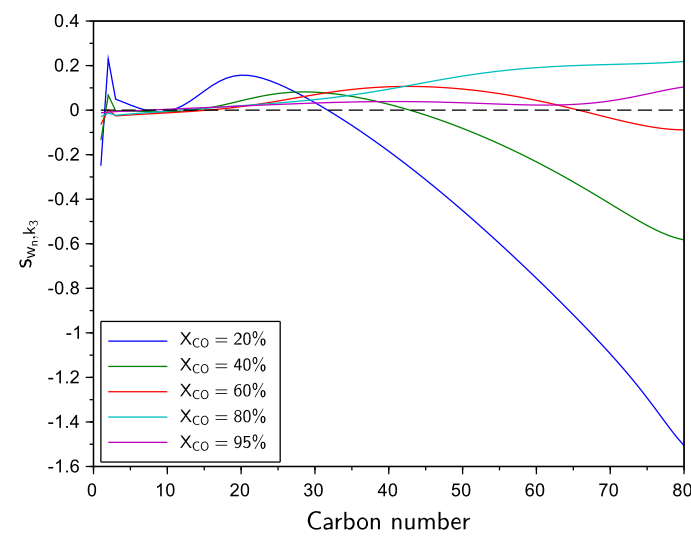

(c) Product distribution sensitivity to $k_{3}$

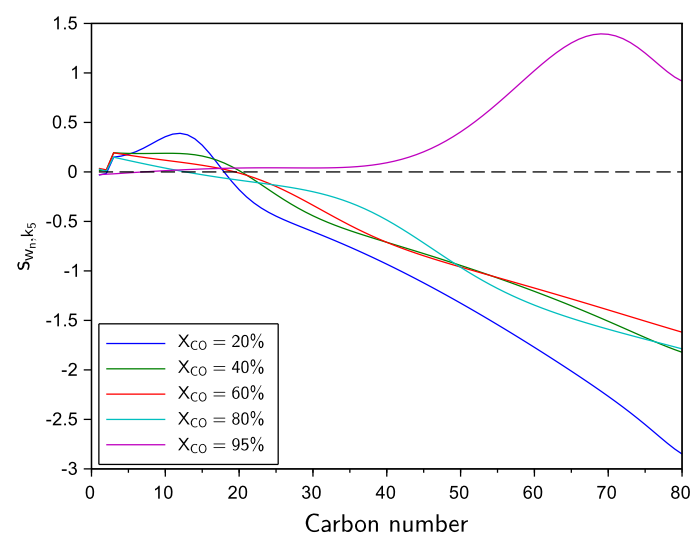

(e) Product distribution sensitivity to $k_{5}$

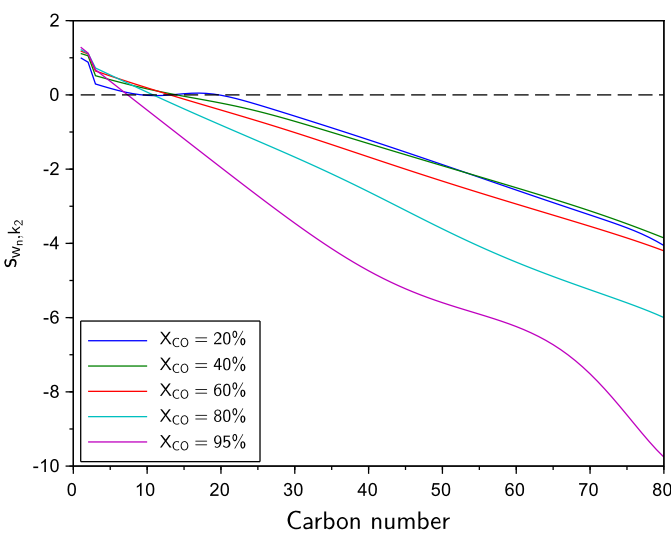

(b) Product distribution sensitivity to $k_{2}$

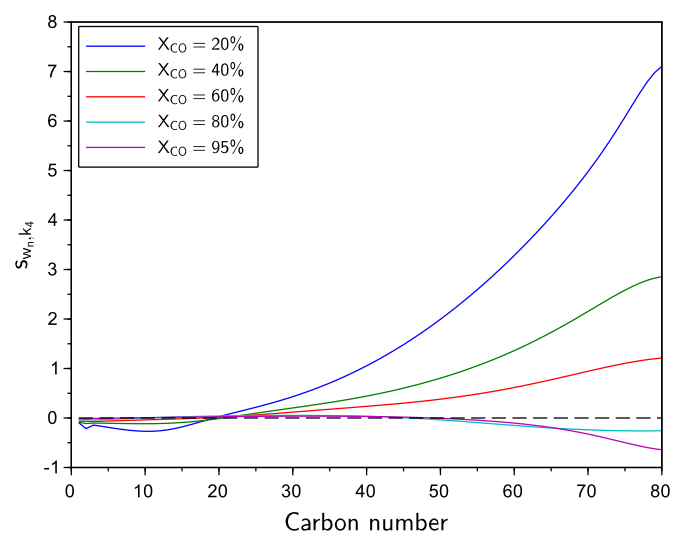

(d) Product distribution sensitivity to $\mathrm{k}_{4}$

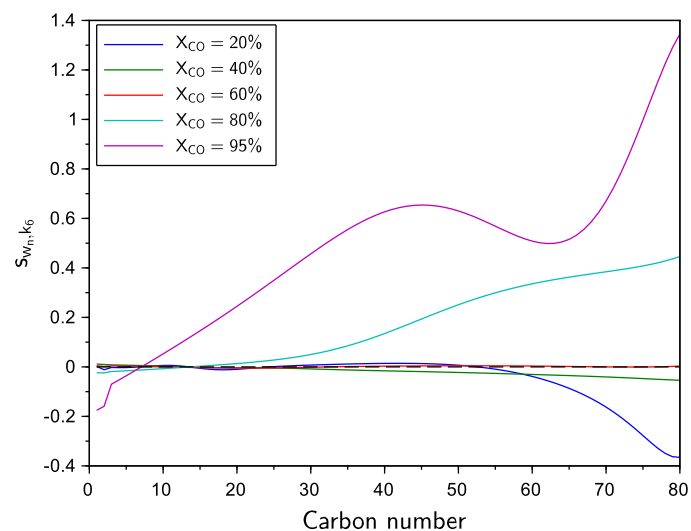

(f) Product distribution sensitivity to $k_{6}$

Figure 5.18: Sensitivity of the product distribution with respect to $\mathrm{k}_{\mathrm{j}}$ as a function of $\mathrm{CO}$ conversion at $220^{\circ} \mathrm{C}, 20 \mathrm{bar}(\mathrm{a})$ and $\mathrm{H}_{2} / \mathrm{CO}$ feed ratio of 2.1 
This analysis indicates that $k_{1}-k_{5}$ can successfully be regressed from data in the form of a product distribution. This is also since no correlation could be found between the rate constants $k_{1}-k_{5}$ when using data of this form. This indicates that $k_{1}-k_{5}$ form a unique set since each of these rate constants has a distinct effect on the product distribution. Meaningful estimates of $k_{1}-k_{5}$ should however be obtained from product distribution data at a CO conversion below $60 \%$. This is the CO conversion level where the product distribution is most sensitive to $k_{1}-k_{5}$. It is also the range over which product distribution data is typically available in literature (Visconti et al., 2007, 2009, 2011; Sage et al., 2014; Todic et al., 2014; Visconti et al., 2016; Kamal K. Pant \& Upadhyayula, 2019: Marchese et al., 2019).

The product distribution is however independent of $\mathrm{k}_{6}$ over this $\mathrm{CO}$ conversion range. No meaningful value of $\mathrm{k}_{6}$ can therefore be estimated. $\mathrm{CO}_{2}$ selectivity data could be used but this is often not reported for this CO conversion range. Consequently, it is necessary to assume that $\mathrm{k}_{6}$ is known in advance. The WGS reaction is often neglected in FT kinetic models but the work of Philippe et al. (2009) and Rafiq et al. (2011) provide an approximation of the WGS rate constant, k6, for Co-based catalysts. This reduces the dimensionality of the parameter estimation process and indicates that meaningful values of $k_{1}-k_{5}$ can be extracted from product distribution data (Schwan, 2001; Sharrock \& Coetzer, 2007).

\subsection{Summary}

A sensitivity analysis on the selectivity to $\mathrm{C}_{1}, \mathrm{C}_{2}, \mathrm{C}_{3}, \mathrm{C}_{4}, \mathrm{C}_{5+}$ and $\mathrm{CO}_{2}$ as function of the rate constants $k_{1}-k_{6}$ was performed to understanding whether these parameters form a unique set. It has been shown that the selectivity characteristics of FT synthesis is most significantly dependent on the rate constants $k_{1}$ and $k_{2}$. This is since these two rate constants determine reactivity along the competing pathways of methanation and chain growth. As such, the sensitivity functions with respect to $k_{1}$ and $k_{2}$ show equal but opposite behaviour. It is also shown that the selectivity to $\mathrm{CO}_{2}$ is more sensitive to $\mathrm{k}_{1}$ and $\mathrm{k}_{2}$ relative to $\mathrm{k}_{6}$ because of their influence on the reactants water and $\mathrm{H}_{2}$. The selectivity curves to $\mathrm{C}_{1}, \mathrm{C}_{2}, \mathrm{C}_{3}, \mathrm{C}_{4}, \mathrm{C}_{5+}$ and $\mathrm{CO}_{2}$ are most sensitive to $\mathrm{k}_{1}$ and $\mathrm{k}_{2}$ across the entire $\mathrm{CO}$ conversion range. The rapid rates of (n-paraffin and 1-olefin) chain growth relative to $\mathrm{CO}$ hydrogenation results in the selectivity to products of carbon number $\mathrm{C}_{5+}$ being most sensitive to $k_{3}-k_{5}$ for low CO conversions (Norval, 2008). The strong dependence of the $\mathrm{CO}_{2}$ selectivity on $\mathrm{k}_{1}$ indicates that this rate constant is not just rate-determining for the $\mathrm{FT}$ reactions, but the WGS reaction too.

The high degree of correlation between the rate constants $k_{1}-k_{6}$ indicates that a simultaneously estimation of all rate constants is not possible when using product selectivity data as a function of 
$\mathrm{CO}$ conversion. Thus, if data in this form is used, it is necessary to replace the current model rate constant set with a reduced model rate constant set. An alternative strategy involves using total hydrocarbon product distribution data at constant CO conversion instead. This is the approach typically followed in literature. The product distribution was shown to be most sensitive to $\mathrm{k}_{1^{-}}$ $k_{5}$, particularly for $\mathrm{CO}$ conversions below $60 \%$. This is also the $\mathrm{CO}$ conversion range over which most data is measured in literature. However, over the same $\mathrm{CO}$ conversion range the product distribution is independent of $k_{6}$. It is therefore necessary to reduce the number of parameters required to be estimated by approximating the value of $k_{6}$ using data in literature. This strategy will allow meaningful values of $k_{1}-k_{5}$ to be extracted from product distribution data at constant CO conversion. 


\section{Chapter 6}

\section{Model Validation}

\subsection{Introduction}

To be useful, the kinetic model developed needs to be compared to experimental data. This involves conducting a quantitative assessment of the predictive ability of the model. This can be achieved by estimating the model parameters i.e. the rate constants through regression to provide an indication of the average value of the response (Montgomery \& Runger, 2014). The high exothermicity of the $\mathrm{FT}$ reactions means that is necessary to know how the rate constants vary with temperature. Therefore, emphasis will be placed on the ability of the model to predict variations in the product distribution with temperature.

Ideally, the effect of temperature on the FT product distribution should be studied experimentally as a function of CO conversion. Unfortunately this is not done in practice. In open literature only the feed conditions, exit CO conversion and the composition of the product distribution, to varying levels of detail are provided. As such, the feed conditions were simulated, with a comparison made between the experimental and model predicted $\mathrm{CO}$ conversion and product distribution. A similar approach is taken in the hydrocracking work of Le Grange (2009).

The work of Visconti et al. (2016) demonstrates that a model developed to describe FT kinetics in a fixed-bed reactor (FBR) can also be used to effectively describe the kinetic behaviour in a slurry reactor. Thus, to ensure that the model is independent of reactor type, both FBR and slurry reactor data will be used to determine the rate constants as a measure of the model's predictive ability. This provides a fair test of the model.

It is necessary to use a consistent dataset in the regression of the model rate constants. The work of Visconti et al. (2007, 2009, 2011) and Visconti et al. (2016) provides the most detailed 
breakdown of the total hydrocarbon distribution in open literature. In each case, a breakdown of the product distribution is provided for products of carbon number from $C_{1}$ to $C_{49}$. In the absence of original experimental work, it is also common in literature to use these datasets to test and develop the model and regress for model rate constants. The quality of these data can thus be seen to be good and as such can provide a fair assessment of the kinetic model developed in this work.

The work of Tucker \& van Steen (2019) studied the effect of CO conversion on lumped product selectivity. This can also be used to assess the model prediction over a range of $\mathrm{CO}$ conversions instead of just the product distribution at a single $\mathrm{CO}$ conversion point. A breakdown of the temperature, pressure and feed ratio of the data used to evaluate the kinetic model is given in Table 6.1.

Table 6.1: Summary of experimental data used to estimate the model rate constants

\begin{tabular}{|c|c|c|c|c|}
\hline Author & Catalyst & Temperature $\left[{ }^{\circ} \mathrm{C}\right]$ & $\begin{array}{l}\text { Pressure } \\
{[\operatorname{bar}(\mathrm{a})]}\end{array}$ & $\begin{array}{l}\mathrm{H}_{2} / \mathrm{CO} \text { feed } \\
\text { ratio }\end{array}$ \\
\hline \multicolumn{5}{|c|}{ Fixed-bed Data } \\
\hline \begin{tabular}{|l|} 
Visconti et al. \\
$(2007,2011)$
\end{tabular} & $\mathrm{Co} / \mathrm{Al}_{2} \mathrm{O}_{3}$ & $220 ; 230 ; 235$ & 20 & $2.1 ; 2.1 ; 2.1$ \\
\hline $\begin{array}{l}\text { Visconti et al. } \\
(2009)\end{array}$ & $\mathrm{Co} / \mathrm{Al}_{2} \mathrm{O}_{3}$ & 220 & 20 & 2.45 \\
\hline \multicolumn{5}{|c|}{ Slurry Reactor Data } \\
\hline \begin{tabular}{|l} 
Visconti et al. \\
$(2016)$
\end{tabular} & $\mathrm{Co} / \mathrm{Al}_{2} \mathrm{O}_{3}$ & 230 & 20 & 2.1 \\
\hline $\begin{array}{l}\text { Tucker \& van } \\
\text { Steen (2019) }\end{array}$ & $\mathrm{Co} / \mathrm{Pt} / \mathrm{Al}_{2} \mathrm{O}_{3}$ & 220 & 10 & 2 \\
\hline
\end{tabular}

\subsection{Parameter Estimation}

In general, the process of parameter estimation involves, firstly, choosing an objective function that measures the difference between the experimental observation and the model prediction (Press et al., 1992). The latter is a function of the choice of rate constants. Secondly, the rate constants are then adjusted until the objective function is at a minimum, which yields the best-fit rate constants (Press et al., 1992).

Typically, the rate constants are estimated using least squares regression. The objective function is given by Equation 6.1. This means that the sum of the residuals squared is minimised, where $Y_{i}$ represents the CO conversion and product weight fractions in this case (Edgar et al., 2001; Heath, 2002: Montgomery \& Runger, 2014). The residual is defined as the vertical difference between 
the experimental observation and model prediction. Thus, it describes the error in the fit of the model to the observation and can be used to provide information about the adequacy of the kinetic model (Montgomery \& Runger, 2014).

$$
f(k)=\sum_{i=1}^{n_{\text {data }}} r_{i}^{2}(k)
$$

where

$$
r_{i}(k)=Y_{e x p, i}-Y_{c a l c, i}(k)
$$

The determination of the best fit rate constants is not the 'end all' of parameter estimation (Press et al., 1992). To be useful, any parameter estimation should, in addition to the estimated rate constants, provide an error estimate on the rate constants and a statistical measure of goodness of fit (Press et al., 1992). The assumption of normality of the residuals and confirmation thereof allows statistical techniques applicable to a normal distribution to be used to evaluate the error in the rate constants and provide a measure of goodness of fit (Montgomery \& Runger, 2014).

In general if the residuals are normally distributed, the residual variance, which is not known in advance, can be estimated using Equation 6.3 (Montgomery \& Runger, 2014). The denominator represents the degrees of freedom, while $m$ represents the number of model parameters (Montgomery \& Runger, 2014). The variance of the rate constants is expressed in terms of the inverse of the matrix $J^{T} J$, where $J$ is the Jacobian 11 of Equation 6.1. The covariance matrix is given by Equation 6.4. The diagonal elements of Equation 6.4 represent the variance of the rate constants and off-diagonal elements are the covariances between rate constants (Montgomery \& Runger, 2014).

$$
\begin{gathered}
\hat{\sigma}^{2}=\frac{\sum_{i=1}^{n_{\text {data }}} r_{i}^{2}}{n_{\text {data }}-m} \\
\operatorname{cov}=\hat{\sigma}^{2}\left(J^{T} J\right)^{-1}
\end{gathered}
$$

The standard error of the rate constants, which is a useful measure of the precision of the estimated rate constants is given by Equation 6.5. where $\operatorname{cov}_{j j}$ refers to the diagonal elements of the covariance matrix. A small standard error implies good precision (Montgomery \& Runger, 2014).

$$
\operatorname{se}\left(\hat{k}_{j}\right)=\sqrt{\operatorname{cov}_{j j}}
$$

It should be noted that the formulae given above are based on least squares minimisation in linear

\footnotetext{
${ }^{1}$ The Jacobian can be calculated using a second order finite difference approximation, using the optimal step size of $\Delta k_{j}=\epsilon_{\text {mach }}^{\frac{1}{3}} k_{j}$.
} 
systems. The integration of mole balances in a FBR or the algebraic solution to the mole balances in a slurry reactor will result in a non-linear system in the rate constants. However, if the model fits the data with a small residua 2 , then the above analyses can be used at least as a first approximation.

\subsection{Solving Procedure}

The process of solving Equation 6.1 requires efficient and robust techniques to determine a solution that is both optimal (minimises the objective function) and feasible (satisfies the physical constraints) (Edgar et al., 2001). Efficiency is important because an iterative solution is required. Trial and error thus become impractical for multi-dimensional problems (Edgar et al., 2001). Robustness is related to the ability to solve the problem and provide confidence that the solution obtained is indeed optimal.

In FT literature, a combination of (meta)heuristic and exact search methods are used to solve Equation 6.1. The motivation behind this choice is that the heuristic search method can explore the full solution space and find the global minimum. The exact search method is then invoked using the result from the heuristic method for refinement of the solution. This approach has been used in the work of Teng et al. (2006); Qian et al. (2013); Todic et al. (2014); Sage et al. (2014); Kamal K. Pant \& Upadhyayula (2019) and Marchese et al. (2019). An exact search method is used in isolation in the work of Wang et al. (2003b); Visconti et al. (2007); Kwack et al. (2011) and Visconti et al. (2011). Generally, the search method algorithms used are the genetic algorithm for the global minimum search and Levenberg-Marquardt algorithm for the local minimum search.

The Levenberg-Marquardt algorithm is a gradient-based method to solve Equation 6.1. It is a modified Newton method which forces the Hessian, matrix of second derivatives, to be positive definite to guarantee convexity (Heath, 2002). The advantage of a positive definite Hessian matrix is that the process of iteration always proceeds 'down-hill'. This ensures that the method is globally convergent to a local minimum. Work on FT kinetic model development, however, does not use the Levenberg-Marquardt method in isolation since it does not find a global minimum, if multiple local minima exist (Todic et al., 2014, Sage et al., 2014; Kamal K. Pant \& Upadhyayula, 2019; Marchese et al. 2019). The genetic algorithm is thus used in conjunction to find the global minimum. This algorithm is a 'population-based' method that relies solely on function evaluations to combine a set of solutions, known as the 'population', to improve the solution through iteration. The search criteria are based on rules that mimic the biological processes of crossover and mutation to improve the solution (Edgar et al., 2001; Fry, 2010).

\footnotetext{
${ }^{2}$ The Hessian is then well approximated by $2 J^{T} J$ i.e. the quadratic approximation to Equation 6.1 is good. This is the Hessian as given by the linear system.
} 
The role of the genetic algorithm is thus to streamline the search for the Levenberg-Marquardt algorithm by providing an initial guess which guarantees convergence (Todic et al., 2014, Sage et al., 2014; Kamal K. Pant \& Upadhyayula, 2019; Marchese et al., 2019). This is since FT kinetic models developed in literature consist of many non-linear terms making it challenging to explore the solution space. The model formulation, therefore, results in Equation 6.1 consisting of multiple stationary points i.e. local minima. This raises the question of model parameter uniqueness, which may be a consequence of the Langmuir-Hinshelwood formation (Sharrock \& Coetzer, 2007).

It would, however, be advantageous to use the Levenberg-Marquardt algorithm only to solve Equation 6.1, as in the work of Wang et al. (2003b); Visconti et al. (2007); Kwack et al. (2011) and Visconti et al. (2011). This requires an understanding of how the model parameters determine the system behaviour which has been established in the sensitivity analysis. The rate constants $k_{1}-k_{5}$ affects the product distribution of FT synthesis. This indicates that only $k_{1}-k_{5}$ can be estimated from experimental data in the form of a product distribution, with the value of $k_{6}$ fixed at an appropriate value. This means that if an initial guess for $k_{1}-k_{5}$ is chosen close to the minimum of Equation 6.1, then the iterative solution process will converge to it (Heath, 2002). The use of the Levenberg-Marquardt algorithm only is appropriate. It is a method that has proven to work well in practice and is the standard for non-linear least squares problems (Press et al., 1992).

A true exact search method involves solving the objective function using multiple different initial guesses scattered through the feasible set to find the global minimum (Edgar et al., 2001; Heath, 2002). If all initial guesses converge to the same result, then it is an indication that the global minimum has been found (Heath, 2002). This is also an indication that the best fit rate constants are unique (Sealy, 1996).

\subsubsection{Method of Solution}

An outline of the steps used to estimate the rate constants $k_{1}-k_{5}$ is given in Figure 6.1. The same basic idea is used in most chemical reaction systems (Teng et al., 2006; Fry, 2010). The solution process begins by manually obtaining an initial guess for the rate constants $k_{1}-k_{5}$. The idea is to start the iterative solution process as close to the desired minimum and hope that the solution process will converge (Heath, 2002). A suitable initial guess should thus yield a response with a similar shape to the experimental product distribution at the target $\mathrm{CO}$ conversion before minimisation of Equation 6.1 can occur.

In the sensitivity analysis, it was shown that the product distribution is independent of $k_{6}$, at least up to a CO conversion of $60 \%$. Consequently, $\mathrm{k}_{6}$ should be set to a reasonable value since the data in Table 6.1 are measured below a CO conversion of 60\%. In FT kinetic model development, 


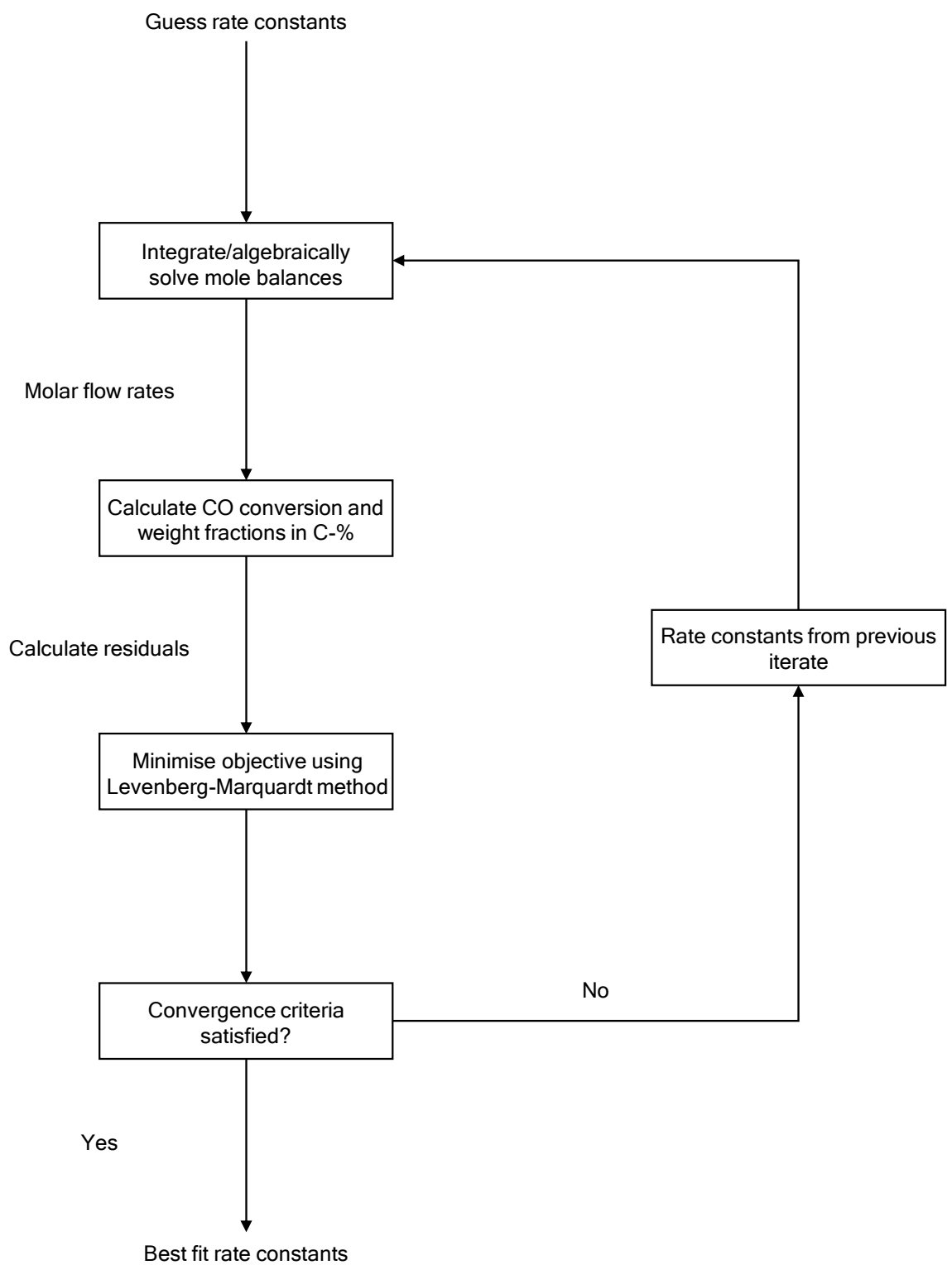

Figure 6.1: Solution process followed to regress the experimental data and determine values of $k_{1}-k_{5}$ 
the water-gas shift (WGS) reaction is often neglected. The work of Philippe et al. (2009) and Rafiq et al. (2011) have considered the WGS reaction and provide an approximation of the WGS rate constant (Equation 6.6) that can be used as an appropriate value of $k_{6}$.

$$
k_{6}=A_{6} \exp \left(-\frac{E_{a, 6}}{R T}\right) \frac{y_{H_{2} O} P_{t o t a l}}{R T}
$$

In Equation 6.6, $A_{6}$ and $E_{a, 6}$ are the pre-exponential factor and activation energy of the WGS reaction, $y_{\mathrm{H}_{2} \mathrm{O}}$ is the vapour phase mole fraction of water, $P_{\text {total }}$ is the total pressure, $R$ is the universal gas constant and $T$ is the temperature. For a $\mathrm{Co} / \mathrm{Al}_{2} \mathrm{O}_{3}$ catalyst, the value of $A_{6}$ and $E_{a, 6}$ are given in Table 6.2 from the work of Rafiq et al. (2011).

Table 6.2: Pre-exponential factor and activation energy used to approximate $k_{6}$ (Rafiq et al., 2011)

\begin{tabular}{lc}
\hline Constant & Value \\
\hline$A_{6} \times 10^{-8}\left[\mathrm{~m}^{3} / \mathrm{kg}_{\text {cat }} / \mathrm{s}\right]$ & 6.805 \\
$E_{a, 6}[\mathrm{~kJ} / \mathrm{mol}]$ & 145 \\
\hline
\end{tabular}

The mole balance equations are then integrated in the case of an FBR or solved algebraically in the case of a slurry reactor by homotopy ${ }^{3}$. In general, kinetic experiments use the same mass of catalyst and vary reactant flow rate to change the residence time. However, when modelling the system it is more advantageous to use the same feed on a mole CO basis and then change the catalyst mass to vary the residence time. The total catalyst mass based on the gas hourly space velocity (GHSV) can be calculated using the conversion in Equation 6.7

$$
\begin{aligned}
& \frac{W_{\text {total }}}{F_{C O}}\left[\frac{\mathrm{kg} \cdot \mathrm{s}}{\mathrm{mo} \mathrm{I}_{\mathrm{CO}}}\right]=\frac{1}{\mathrm{GHSV}}\left[\frac{g_{\text {cat }} \cdot \mathrm{h}}{\mathrm{Nml}}\right] \times \frac{100 \mathrm{~cm}^{3}}{1 \mathrm{~m}^{3}} \times V_{S T P}\left[\frac{\mathrm{m}^{3}}{\mathrm{~mol}}\right] \\
& \times \frac{1+\mathrm{H}_{2} / \text { CO ratio }}{1 \mathrm{~mol}_{\mathrm{CO}}} \times \frac{1 \mathrm{~kg}_{\text {cat }}}{1000 \mathrm{~g}_{\text {cat }}} \times \frac{3600 \mathrm{~s}}{1 \mathrm{~h}}
\end{aligned}
$$

The output from solving the mole balances is the exit molar flow rate which is used to calculate the model predicted $\mathrm{CO}$ conversion and product weight fractions on a carbon basis. The residual can then be estimated as the difference between the experimental and model predicted $\mathrm{CO}$ conversion and product weight fractions. The residuals are minimised using the Levenberg-Marquardt method, which provides the next iterate for $k_{1}-k_{5}$. This process continues until the predefined convergence criteria of the solver is achieved. The output of the solution process is the best fit values of $k_{1}-k_{5}$.

When fitting the model to the data, care was taken to determine whether the output was unique. This is done by varying the initial guess over a range of starting guesses within the feasible set

\footnotetext{
${ }^{3}$ This involves using the solution of an 'easier-to-solve' system as initialisation to solve the more challenging system.
} 
(Sealy, 1996; Heath, 2002). It was decided to vary each rate constant $k_{1}-k_{5}$ by $1 \%$ above and below their initial guess values. The motivation behind this is that if $k^{o p t}$ is at least the local minimum, then no other point $k$ in the neighbourhood of $k^{o p t}$ should yield a value of the objective function $f(k)$ less than $f\left(k^{o p t}\right)$ i.e. $f(k) \geq f\left(k^{o p t}\right)$ (Edgar et al., 2001). As an additional check Sealy (1996) recommends replacing the experimental data with model data. If the original solution was unique, then the steps in Figure 6.1 should produce the rate constants $k_{1}-k_{5}$ that generated the data (Sealy, 1996).

\subsubsection{Model Assumptions}

The following assumptions are made for the FBR model:

1. Isothermal conditions.

2. Isobaric conditions.

3. Ideal plug flow.

4. Negligible internal and external mass transfer effects.

5. Constant catalyst activity.

6. Reaction in the vapour phase only.

Assumption 1 is made because the catalyst is sufficiently diluted with alumina or silica. This allows the measured axial temperature to be kept within $\pm 2{ }^{\circ} \mathrm{C}$ of the reaction temperature (Visconti et al. 2007, 2009, 2011). Kwack et al. (2011) included an energy balance in their reactor model equation set when regressing for model rate constants in their kinetic model development and reported a maximum temperature rise of $5{ }^{\circ} \mathrm{C}$ for all reaction conditions. The temperature profile is thus almost constant and can be suitably approximated by assuming isothermal conditions.

Assumption 4 is made because small catalyst particles of diameter $10^{1}-10^{2} \mu \mathrm{m}$ are used. Both external and internal mass transfer effects are thus minimised (Todic et al., 2014). Assumption 5 is necessary to ensure that the values of $k_{1}-k_{5}$ determined by regression will be reflective of typical reactor operation.

Assumption 6 applies since the amount of liquid products formed is negligible in comparison to the vapour phase products at low CO conversion (Visconti, 2014). At higher CO conversion, the use of species activity as the kinetic driving force means that the magnitude of the rate constants determined will be independent of phase and as such can be regressed assuming vapour phase conditions (Mthombeni, 2009). Assumption 6 will, however, be checked by performing a flash 
calculation of the reactor product. Equation 6.8 can thus be used in the regression, where $F_{i}$ is the molar flow rate, $x$ is the normalised catalyst weight, $r_{i}$ is the rate of reaction, $a_{i}$ is the species activity and $W_{\text {total }}$ is the total catalyst weight.

$$
\frac{d F_{i}}{d x}=r_{i}\left(a_{i}, k_{j}\right) W_{\text {total }}
$$

In addition to Assumption 1, 2, 4, 5 and 6 above, the following assumptions are made for the slurry reactor model:

1. Negligible concentration gradients. Perfect mixing can thus be assumed with the catalyst uniformly distributed in the reactor (Schulz \& Claeys, 1999).

2. Negligible internal and external mass transfer effects (Todic et al., 2014).

Assumption 1 is made because the slurry reactor rig is typically well-agitated to reduce the effects of channelling (Todic et al., 2014). Assumption 2 is made because of the homogeneous operating regime of the slurry reactor. This is since the feed to laboratory slurry reactors needs to enter at low linear gas velocities to avoid slugging problems (Sie \& Krishna, 1999). Consequently, this reduces external mass transfer limitations between the sparged gas and wax-catalyst slurry. Equation 6.9, the design equation for a continuously stirred-tank reactor (CSTR), can thus be used in the regression, where $F_{i, 0}$ is the feed flow rate of species $i$.

$$
\frac{F_{i, 0}-F_{i}}{W_{\text {total }}}+r_{i}\left(a_{i}, k_{j}\right)=0
$$

\subsection{Results and Discussion: Fixed-Bed Reactor Data}

\subsubsection{Data of Visconti et al. $(2007,2011)$}

Visconti et al. (2007, 2011) have done extensive work on the development of kinetic models for the FT reaction system. The data in their papers also provides the most detailed analysis of the total hydrocarbon product distribution up to $\mathrm{C}_{49}$ in open literature. The model fit to the data of Visconti et al. (2007, 2011) is shown in Figure 6.2. In each case, the model product distribution is given up to products of carbon number $\mathrm{C}_{80}$ which has been shown to be an appropriate choice to represent the product distribution of $\mathrm{FT}$ synthesis. Although data is only provided up to $\mathrm{C}_{49}$, this will in addition provide an indication of the model prediction of the quantity of products of higher carbon number.

In Figure 6.2, at all conditions the model fit to the total hydrocarbon product distribution and 

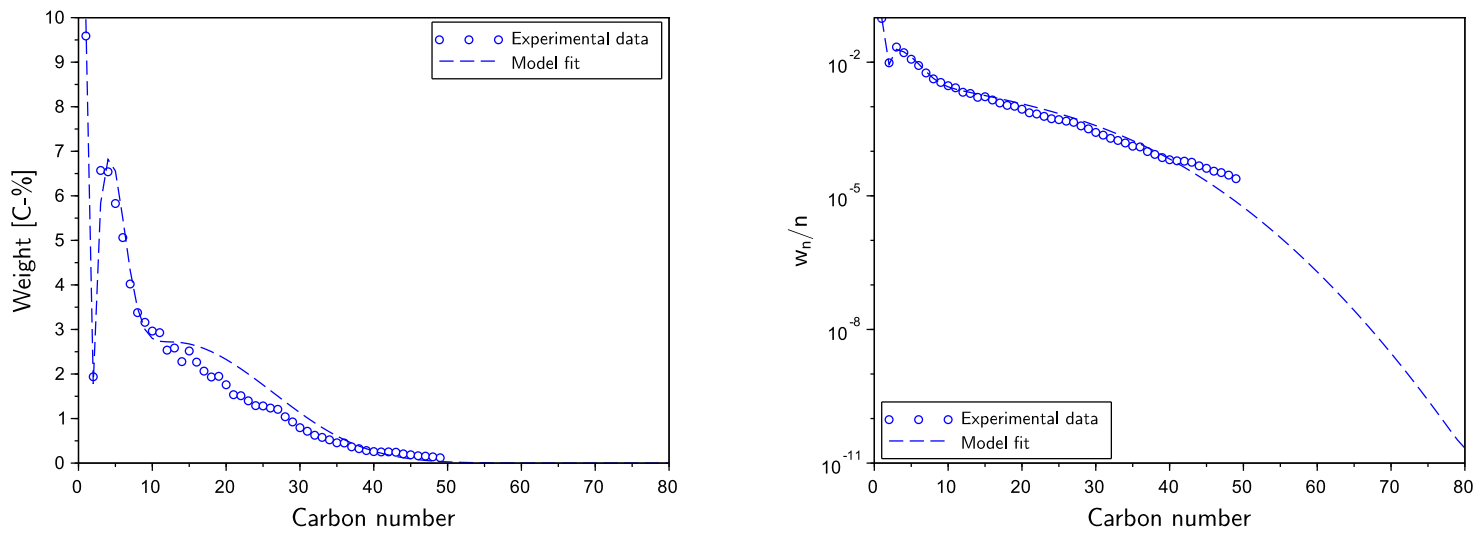

(a) Product distribution: $220^{\circ} \mathrm{C}, 20$ bar(a), $\mathrm{H}_{2} / \mathrm{CO}$ (b) (b) ASF distribution: $220^{\circ} \mathrm{C}, 20$ bar(a), $\mathrm{H}_{2} / \mathrm{CO}$ feed feed ratio of 2.1 ratio of 2.1
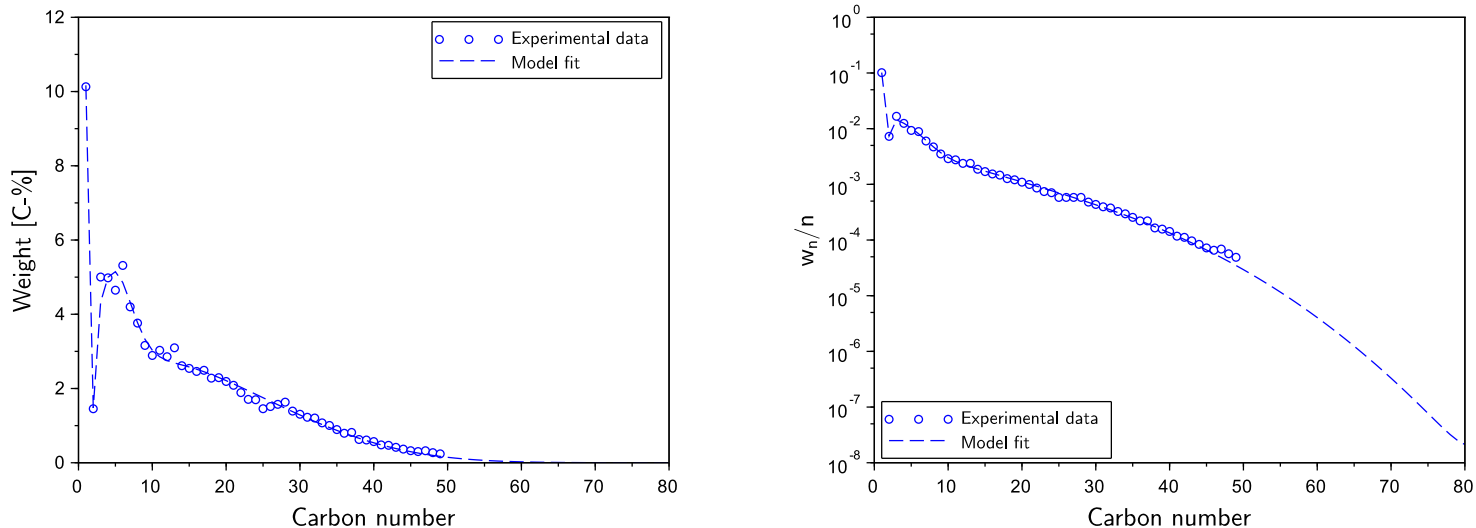

(c) Product distribution: $230^{\circ} \mathrm{C}, 20$ bar(a), $\mathrm{H}_{2} / \mathrm{CO}$ (d) ASF distribution: $230^{\circ} \mathrm{C}, 20$ bar(a), $\mathrm{H}_{2} / \mathrm{CO}$ feed feed ratio of 2.1 ratio of 2.1
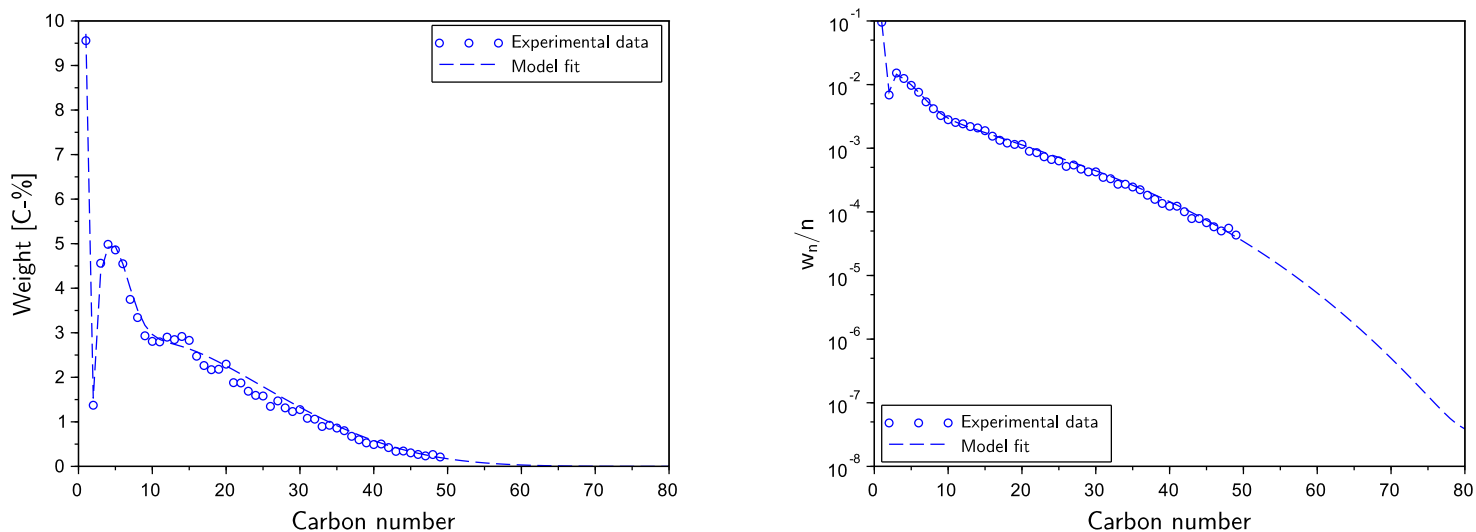

(e) Product distribution: $235^{\circ} \mathrm{C}, 20$ bar(a), $\mathrm{H}_{2} / \mathrm{CO}$ (f) ASF distribution: $235^{\circ} \mathrm{C}, 20$ bar(a), $\mathrm{H}_{2} / \mathrm{CO}$ feed feed ratio of 2.1 ratio of 2.1

Figure 6.2: Model fit to the data of Visconti et al. (2007, 2011) at a GHSV of $5000 \mathrm{Nml} / \mathrm{g}_{\text {cat }} / \mathrm{h}$ 
ASF distribution is good. At a temperature of $220^{\circ} \mathrm{C}$, the model fit is best for products of carbon number between $\mathrm{C}_{2}-\mathrm{C}_{10}$. This may arise because of the low $\mathrm{CO}$ conversion of the data (see Table 6.3), where the product distribution will consist mainly of 1-olefins at carbon numbers between $C_{2}{ }^{-}$ $\mathrm{C}_{10}$. Consequently, the good model fit to the lower carbon number end of the distribution causes deviations between the data and model prediction. This is evident in Figure $6.2 \mathrm{~b}$ for products of carbon number $\mathrm{C}_{40}$ and greater. Regardless, the description of the trend in the data by the model is good at these conditions.

An improvement in the model fit to the product distribution at all carbon numbers is achieved for increases in temperature to $230^{\circ} \mathrm{C}$ and $235^{\circ} \mathrm{C}$ in Figure $6.2 \mathrm{C}$ and $6.2 \mathrm{e}$. This arise because of an increase in $\mathrm{CO}$ conversion (see Table 6.3) enabling a shift in the product distribution to products of higher carbon number. This provides confidence in the model extrapolation to and approximation of the quantities of products of carbon number up to $C_{80}$. This is observed as the good prediction by the model of the tail end of the product distribution in Figure $6.2 \mathrm{~d}$ and $6.2 \mathrm{f}$

In Table 6.3, a comparison is made between the experimental and model predicted CO conversion. In each case, the model can effectively describe the rate of $\mathrm{CO}$ consumption corresponding to the respective product distributions in Figure 6.2. This is shown by the small relative error between the experimental and model predicted $\mathrm{CO}$ conversions. The improved fit to the product distribution at $230^{\circ} \mathrm{C}$ and $235^{\circ} \mathrm{C}$ corresponds to an improved prediction of the $\mathrm{CO}$ conversion. The assumption of purely vapour phase conditions is confirmed by the high vapour fraction, which remained above 0.99 in each case. However, with increases in $\mathrm{CO}$ conversion, there is a slight decrease in the vapour fraction indicating that neglecting liquid formation in the kinetic model is dependent on the operating CO conversion.

Table 6.3: Comparison between the experimental and model predicted $\mathrm{CO}$ conversion and estimated vapour fraction for the data of Visconti et al. (2007, 2011)

\begin{tabular}{ccccc}
\hline Temperature $\left[{ }^{\circ} \mathrm{C}\right]$ & $\mathrm{X}_{\mathrm{CO}, \exp }[\%]$ & $\mathrm{X}_{\mathrm{CO}, \text { model }}[\%]$ & Relative error $[\%]$ & Vapour fraction $[-]$ \\
\hline 220 & 14.18 & 13.99 & 1.340 & 0.9997 \\
230 & 26.58 & 26.55 & 0.1129 & 0.9990 \\
235 & 30.99 & 30.96 & 0.09681 & 0.9988 \\
\hline
\end{tabular}

A quantitative measure of the goodness of the model fit to the data in Figure 6.2 is provided by the estimated rate constants at each temperature in Table 6.4. The resulting estimate of the rate constants was in each case found to be unique as the same values of $k_{1}-k_{5}$ were obtained upon variation of the starting guess to the solution process in Figure 6.1. In addition, each rate constant is estimated with a small standard error. This provides confidence in the values in Table 6.4. reflected by the good model fit in Figure 6.2. 
The estimated value of $k_{1}$ is the smallest in magnitude relative to $k_{2}-k_{5}$. This confirms that $C O$ hydrogenation must be rate-determining for the FT reaction system (van Helden et al., 2017; Allie \& Nyathi, 2019: Hensen et al., 2020). The more rapid reaction steps 2-5 relative to reaction step 1 in conjunction with the equilibrium constants of these reactions contributes to the observed characteristics in Figure 6.2. The kinetic modelling of the FT reaction system using an equilibrium basis is thus an effective strategy to predict the rates of reaction and the character of the product distribution, which is evident in Figure 6.2. This agrees with the hypothesis of Norval \& Phillips (1990); Norval (2008).

Table 6.4: Summary of the estimated rate constants resulting from the data of (Visconti et al. 2007, 2011)

\begin{tabular}{cccc}
\hline Rate constant $[\mathrm{mol} / \mathrm{kg} / \mathrm{s}]$ & $220^{\circ} \mathrm{C}$ & $230{ }^{\circ} \mathrm{C}$ & $235^{\circ} \mathrm{C}$ \\
\hline$k_{1} \times 10^{6}$ & $2.527 \pm 0.072$ & $5.192 \pm 0.043$ & $6.184 \pm 0.041$ \\
$k_{2} \times 10^{5}$ & $1.562 \pm 0.083$ & $4.179 \pm 0.160$ & $4.933 \pm 0.174$ \\
$k_{3} \times 10^{3}$ & $1.728 \pm 0.156$ & $2.505 \pm 0.176$ & $2.832 \pm 0.189$ \\
$k_{4} \times 10^{2}$ & $7.651 \pm 0.676$ & $16.64 \pm 1.50$ & $18.20 \pm 1.63$ \\
$k_{5} \times 10^{3}$ & $4.615 \pm 0.494$ & $8.662 \pm 0.760$ & $7.723 \pm 0.650$ \\
\hline
\end{tabular}

\subsubsection{Data of Visconti et al. (2009)}

The work of Visconti et al. (2009) compared the product distributions obtained from $\mathrm{CO}$ and $\mathrm{CO}_{2}$ hydrogenation. The model fit to the product distribution obtained from $\mathrm{CO}$ hydrogenation is shown in Figure 6.3. The product distribution data contained products of carbon number up to $C_{49}$. As before, the model product distribution contained products of carbon number up to $\mathrm{C}_{80}$ to provide an indication of the prediction of the quantity of products of higher carbon number.

In Figure 6.3, a good fit to the data is obtained, particularly for the products of low carbon number. The good fit to the product distribution for products of low carbon number, however, resulted in discrepancies between the data and model predicted product distribution for products of higher carbon number at around $\mathrm{C}_{40}$. This is seen in Figure 6.3b. Similar behaviour was found when the model was fit to the data of Visconti et al. (2007, 2011) at $220^{\circ} \mathrm{C}$ in Figure 6.2a and 6.2b. At the CO conversion of the data (see Table 6.5), the product distribution consists of mainly 1-olefins at the lower carbon number end of the product distribution. In addition, the $\mathrm{H}_{2} / \mathrm{CO}$ feed ratio of the data favours the formation of products of low carbon number. Together with the good fit of the model to the product distribution for the low carbon number products, this indicates that the product distribution data for products of carbon number $\mathrm{C}_{40}$ and greater might be in error and that the model provides a more appropriate approximation of the quantity of these products. Regardless, the trend in the product distribution data is represented adequately by the model. 


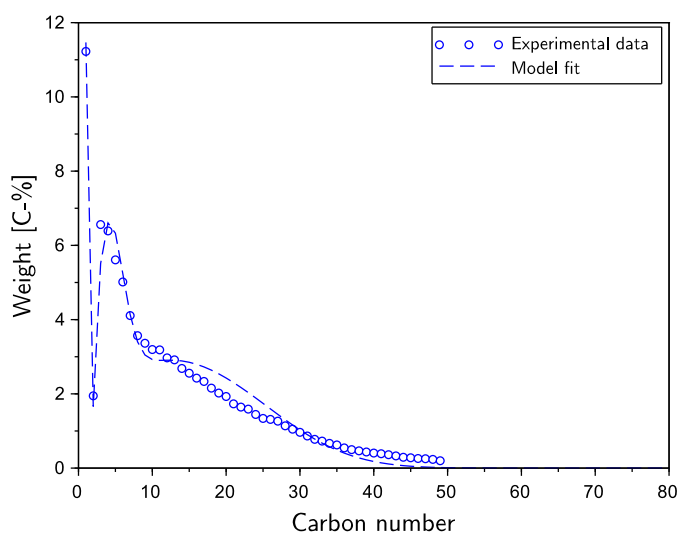

(a) Product distribution: $220^{\circ} \mathrm{C}, 20 \mathrm{bar}(\mathrm{a}), \mathrm{H}_{2} / \mathrm{CO}$ feed ratio of 2.45

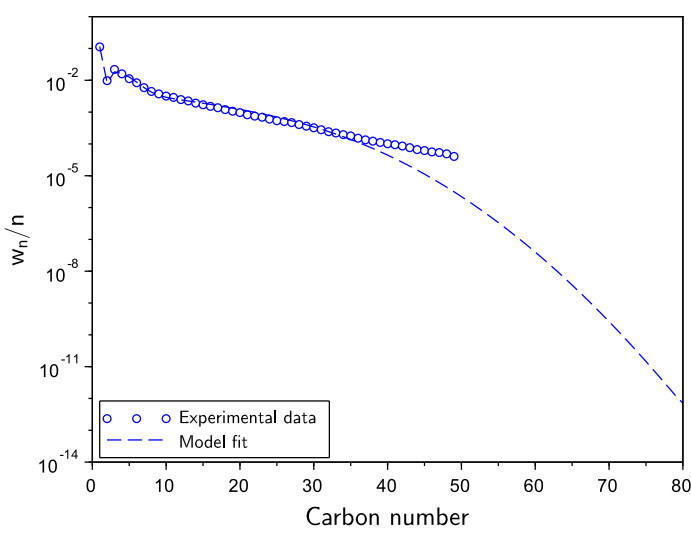

(b) ASF distribution: $220^{\circ} \mathrm{C}, 20$ bar(a), $\mathrm{H}_{2} / \mathrm{CO}$ feed ratio of 2.45

Figure 6.3: Model fit to the data of Visconti et al. (2009) at a GHSV of $4800 \mathrm{Nml} / \mathrm{gcat}_{\text {cat }} / \mathrm{h}$

In Table 6.5 a comparison is made between the experimental and model predicted CO conversion. Good agreement was found shown by the small relative error between the experimental and model predicted values. The model thus provides a good approximation of the consumption of CO in FT synthesis. The assumption of a vapour phase reaction is also shown to be applicable by the vapour fraction in Table 6.5 which is reflective of a negligible liquid content in the reactor.

Table 6.5: Comparison between the experimental and model predicted $\mathrm{CO}$ conversion and estimated vapour fraction for the data of Visconti et al. (2009)

\begin{tabular}{ccccc}
\hline Temperature $\left[{ }^{\circ} \mathrm{C}\right]$ & $\mathrm{X}_{\mathrm{CO}, \exp }[\%]$ & $\mathrm{X}_{\mathrm{CO}, \text { model }}[\%]$ & Relative error [\%] & Vapour fraction $[-]$ \\
\hline 220 & 22.00 & 21.88 & 0.5455 & 0.9995 \\
\hline
\end{tabular}

A quantitative measure of the goodness of the model fit to the product distribution data of Visconti et al. (2009) is the estimated rate constants and their respective error estimates. These values are given in Table 6.6. The resulting estimates of the rate constants were found to be unique as the same values of $k_{1}-k_{5}$ were obtained upon variation of the starting guess to the solution process in Figure 6.1. In addition, each rate constant is estimated with a small standard error. This provides confidence in the values in Table 6.6.

The estimated rate constant values in Table 6.6 are of the same order of magnitude as the values obtained in Table 6.4 at $220^{\circ} \mathrm{C}$. This indicates consistency of the model in its ability to describe the product distribution data from different literature sources at the same temperature. The differences which arise in the actual numerical values between the two datasets can be attributed to the different residence time, $\mathrm{H}_{2} / \mathrm{CO}$ feed ratio and variations in the properties of the $\mathrm{Co} / \mathrm{Al}_{2} \mathrm{O}_{3}$ 
catalyst samples which affects the resulting rate constant estimates.

Table 6.6: Summary of the estimated rate constants resulting from the data of (Visconti et al., 2009)

\begin{tabular}{cc}
\hline Rate constant $[\mathrm{mol} / \mathrm{kg} / \mathrm{s}]$ & $220^{\circ} \mathrm{C}$ \\
\hline$k_{1} \times 10^{6}$ & $3.613 \pm 0.065$ \\
$k_{2} \times 10^{5}$ & $1.956 \pm 0.099$ \\
$k_{3} \times 10^{3}$ & $1.244 \pm 0.100$ \\
$k_{4} \times 10^{2}$ & $5.427 \pm 0.421$ \\
$k_{5} \times 10^{3}$ & $3.369 \pm 0.345$ \\
\hline
\end{tabular}

\subsection{Results and Discussion: Slurry Reactor Data}

\subsubsection{Data of Visconti et al. (2016)}

The work of Visconti et al. (2016) aimed to investigate whether a slurry reactor or a FBR is more appropriate for kinetic measurements in FT synthesis. One of the results of this work is that a kinetic model developed in a FBR can equally well be used to describe the FT reaction in a slurry reactor. This provides an additional test of the kinetic model developed in this work to ensure that it is independent of reactor type. The product distribution data contained products of carbon number up to $\mathrm{C}_{49}$. As before, the model product distribution contained products of carbon number up to $\mathrm{C}_{80}$ to provide an indication of the prediction of the quantity of products of higher carbon number.
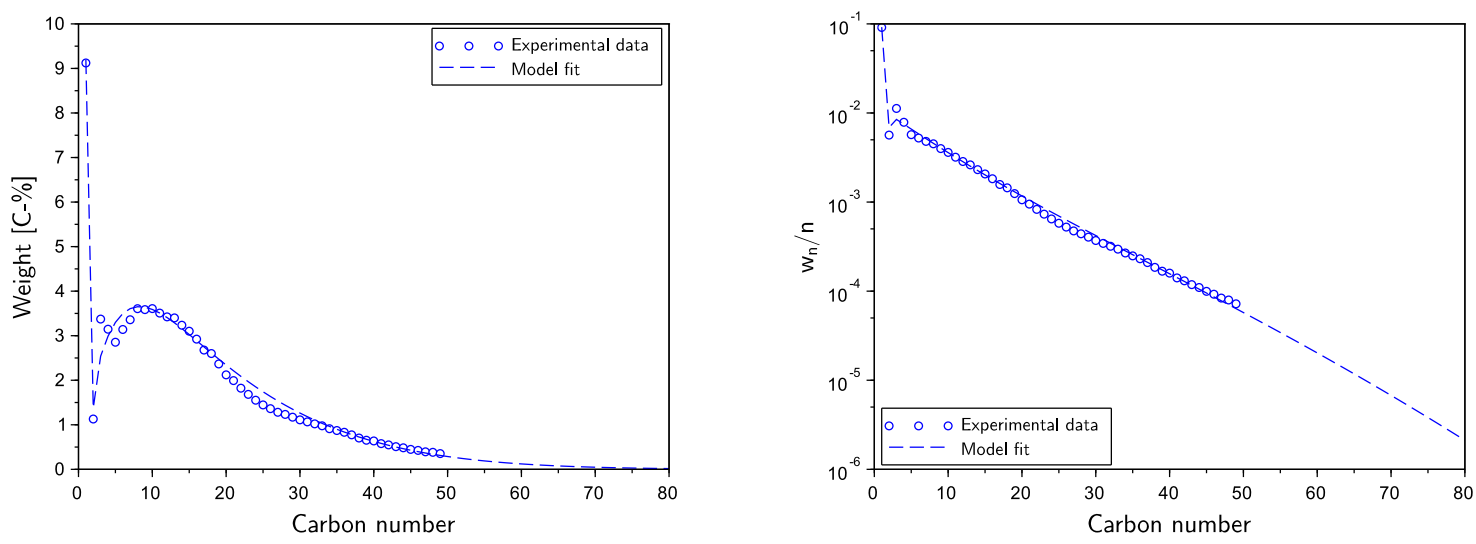

(a) Product distribution: $230^{\circ} \mathrm{C}, 20$ bar(a), $\mathrm{H}_{2} / \mathrm{CO}$ (b) ASF distribution: $230^{\circ} \mathrm{C}, 20$ bar(a), $\mathrm{H}_{2} / \mathrm{CO}$ feed feed ratio of 2.1 ratio of 2.1

Figure 6.4: Model fit to the data of Visconti et al. (2016) at a GHSV of $2000 \mathrm{Nml} / \mathrm{g}_{\mathrm{cat}} / \mathrm{h}$ 
A comparison of the experimental and model-predicted product distributions is shown in Figure 6.4. From Figure 6.4a, the model can effectively predict the product distribution at all carbon numbers. In Figure 6.4b, the data follows a linear relationship, which is also evident in the model prediction. The validity of the ASF distribution thus appears to be more valid in a slurry reactor. This, however, may be a consequences of the operating CO conversion of the data (see Table 6.7), where the product distribution consists mostly of $n$-paraffins. The good qualitative prediction of the data indicates that the kinetic model developed in this work is independent of reactor type. This agrees with the findings of Visconti et al. (2016).

In Table 6.7, the model provides a good prediction of the $\mathrm{CO}$ conversion. This is shown by the small relative error between these two quantities. Despite the high CO conversion, the estimated vapour fraction of the reactor product is reflective of purely vapour phase conditions. The good description and fit to data using only vapour phase conditions indicates that the reaction occurs mainly in the vapour phase. The presence of the wax inside the reactor, which forms a slurry with the catalyst thus acts only as a diluent to maintain isothermal conditions during reactor operation, and as such does not affect product formation and selectivity in FT synthesis.

Table 6.7: Comparison between the experimental and model predicted $\mathrm{CO}$ conversion and estimated vapour fraction for the data of Visconti et al. (2016)

\begin{tabular}{ccccc}
\hline Temperature $\left[{ }^{\circ} \mathrm{C}\right]$ & $\mathrm{X}_{\mathrm{CO} \text {, exp }}[\%]$ & $\mathrm{X}_{\mathrm{CO}, \text { model }}[\%]$ & Relative error [\%] & Vapour fraction [-] \\
\hline 230 & 47.84 & 47.79 & 0.1045 & 0.9975 \\
\hline
\end{tabular}

The values of the estimated rate constants are given in Table 6.8. While a good qualitative fit to the data is achieved in Figure 6.4a and 6.4b, the resulting estimates of the rate constants have larger errors, particularly for $\mathrm{k}_{3}-\mathrm{k}_{5}$. This arises because of the $\mathrm{CO}$ conversion corresponding to the product distribution data. At this $\mathrm{CO}$ conversion level, the product distribution predicted by the model is less sensitive to $k_{3}-k_{5}$. The product distribution consists mainly of $n$-paraffins at this $C O$ conversion which will make it challenging to estimate a value of $k_{5}$, the 1-olefin chain growth rate constant. This is reflected by the high error in the estimate of $k_{5}$.

Table 6.8: Summary of the estimated rate constants resulting from the data of Visconti et al. (2016)

\begin{tabular}{cc}
\hline Rate constant $[\mathrm{mol} / \mathrm{kg} / \mathrm{s}]$ & $230^{\circ} \mathrm{C}$ \\
\hline$k_{1} \times 10^{6}$ & $5.501 \pm 0.046$ \\
$k_{2} \times 10^{5}$ & $2.445 \pm 0.825$ \\
$k_{3} \times 10^{3}$ & $0.3961 \pm 0.2869$ \\
$k_{4} \times 10^{2}$ & $1.436 \pm 0.544$ \\
$k_{5} \times 10^{3}$ & $5.933 \pm 1.661$ \\
\hline
\end{tabular}


This emphasises the importance of providing an error estimate in the parameters which result from the regression of data. The results in Figure $6.4 \mathrm{a}$ and $6.4 \mathrm{~b}$ may be misleading regarding the meaningfulness of a parameter estimate. It also emphasises the importance of a sensitivity analysis and performing data regression at a CO conversion where the product distribution is most sensitive to the model rate constants.

\subsubsection{Data of Tucker \& van Steen (2019)}

Ideally, the kinetic model needs to be validated by fitting the model to product distribution data as a function of $\mathrm{CO}$ conversion. In FT kinetic model development literature this is not done in practice. The work of Tucker \& van Steen (2019) has studied the influence of CO conversion on the selectivity characteristics of the FT reaction system. These data can be used to assess the model predicted product selectivity as a function of $\mathrm{CO}$ conversion.

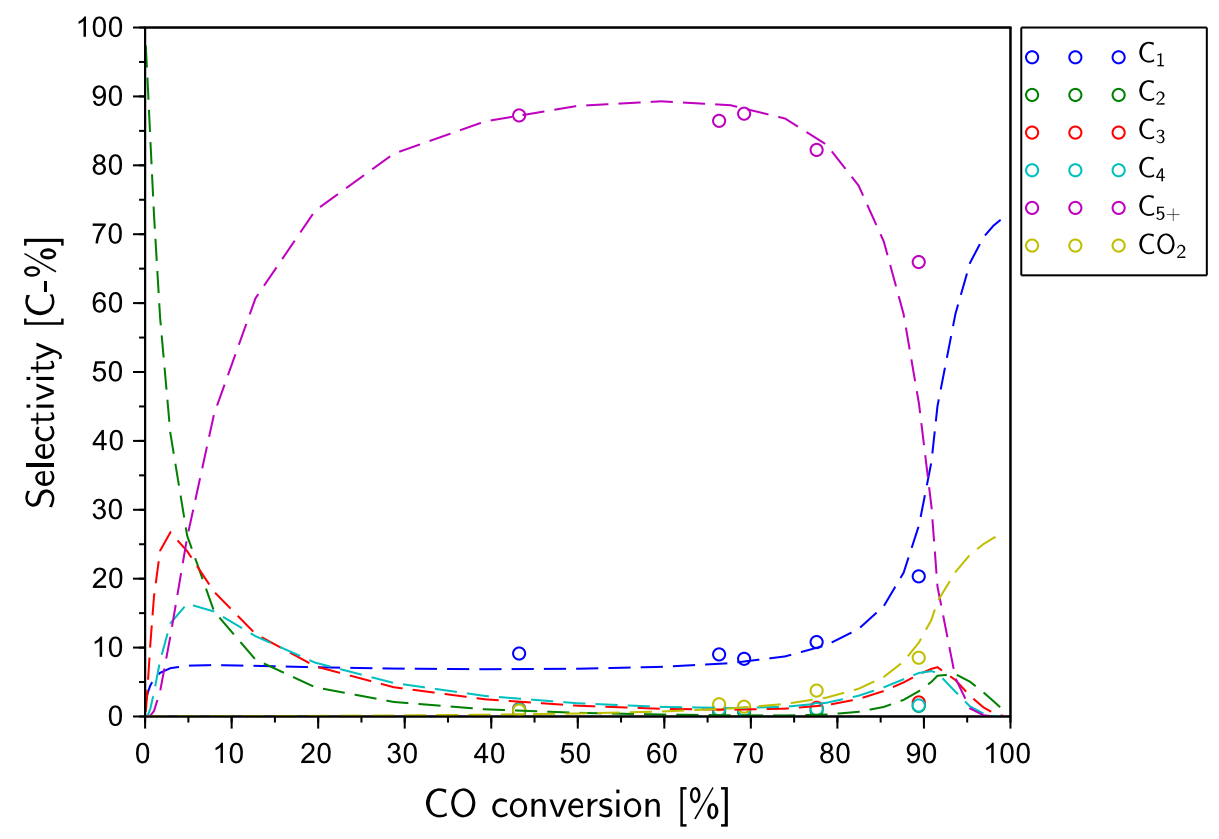

Figure 6.5: Model fit to the data of Tucker \& van Steen (2019)

In Figure 6.5 a comparison is made between the model predicted and experimental $C_{1}, C_{2}, C_{3}, C_{4}$, $\mathrm{C}_{5+}$ and $\mathrm{CO}_{2}$ selectivity. The rate constants that result in this case are arbitrary to illustrate the effectiveness of the equilibrium-control in the model to predict product selectivity as a function of $\mathrm{CO}$ conversion. The value of the rate constants $\mathrm{k}_{1}-\mathrm{k}_{6}$ are given in Table 6.9. From Figure 6.5, this strategy allows the model to effectively predict the trend in the product selectivity with increasing 
CO conversion. Deviations from the trends in the data occur for the $C_{2}, C_{3}$ and $C_{4}$ selectivity, with a good approximation made of the $\mathrm{C}_{1}, \mathrm{C}_{5+}$ and $\mathrm{CO}_{2}$ selectivity profiles.

Table 6.9: Summary of the rate constants used to estimate the selectivity profiles of Tucker \& van Steen (2019)

\begin{tabular}{cc}
\hline Rate constant $[\mathrm{mol} / \mathrm{kg} / \mathrm{s}]$ & $220^{\circ} \mathrm{C}$ \\
\hline$k_{1}$ & 0.002364 \\
$k_{2}$ & 0.0012 \\
$k_{3}$ & 0.001 \\
$k_{4}$ & 8 \\
$k_{5}$ & 0.8 \\
$k_{6}$ & 0.0001 \\
\hline
\end{tabular}

Tucker \& van Steen (2019) report two distinct operating regimes with respect to CO conversion, although the second is based only on the product selectivity at a CO conversion of $90 \%$. This is shown in Figure 6.5. Initially, between $\mathrm{CO}$ conversions of $40-75 \%$, the selectivity to $C_{1}$ and $C_{5+}$ are invariant with changes in $\mathrm{CO}$ conversion. However, beyond a $\mathrm{CO}$ conversion of $75 \%$, the rise in $\mathrm{CO}_{2}$ selectivity corresponds to a rise in $\mathrm{C}_{1}$ and a consequent decline in $\mathrm{C}_{5+}$ selectivity.

The change in the selectivity profiles predicted by the model is a result of the reverse chain growth reactions being favoured. Consequently, there is a shift in selectivity from products of high carbon number to products of low carbon number. In addition, as $\mathrm{CO}$ reacts to form the monomer $\mathrm{CH}_{2}$, water also forms. This favours the forward WGS reaction leading to the formation of $\mathrm{CO}_{2}$ and $\mathrm{H}_{2}$. The increase in $\mathrm{H}_{2}$ formation favours the hydrogenation of $\mathrm{CH}_{2}$. The preferential consumption of $\mathrm{CH}_{2}$ by hydrogenation favours the reverse chain growth reactions. Not only does this result in a rise in methane selectivity, but it also favours selectivity towards shorter chain products. This is observed as the rise in $C_{2}, C_{3}$ and $C_{4}$ selectivity in Figure 6.5. Ultimately, the system is tending towards a thermodynamically favoured methane product at complete $\mathrm{CO}$ conversion as shown in Figure 6.5 (Subiranas, 2009).

The good prediction of the product selectivity across a range of $\mathrm{CO}$ conversions by the model indicates that its behaviour is physically consistent and that it can be used to determine an operating $\mathrm{CO}$ conversion where an optimal $\mathrm{C}_{5+}$ selectivity is obtained, the goal in any synthetic crude production plant.

\subsection{Rate Constant Temperature Dependence}

A temperature-dependent expression for each rate constant $k_{1}-k_{5}$ can be obtained by fitting the estimated values of $k_{1}-k_{5}$ at each temperature to the Arrhenius expression, Equation 6.10. The 
most consistent rate constant estimates were obtained upon regression of the data of Visconti et al. (2007, 2011). As such, these values were used to estimate the pre-exponential factor and activation energy for each rate constant.

$$
k_{j}=A_{j} \exp \left(-\frac{E_{a, j}}{R T}\right)
$$

From Figure 6.6, Equation 6.10 fits the rate constant estimates from the data of Visconti et al. (2007, 2011) well. The data of Visconti et al. (2009) and Visconti et al. (2016) represent outlier points at $220^{\circ} \mathrm{C}$ and $230^{\circ} \mathrm{C}$ respectively. This is attributed to the different residence time under which these experiments are performed compared to the data of Visconti et al. (2007, 2011). This may suggest that the rate constant estimates depend on CO conversion at constant temperature. This assertion can, however, only be validated if more data is considered. This highlights the importance of studying the product distribution isothermally, at a range of temperatures, as a function CO conversion. The fit to the data of Visconti et al. (2007, 2011) and the resulting estimates of the pre-exponential factors and activation energies should therefore be taken only as a first approximation.

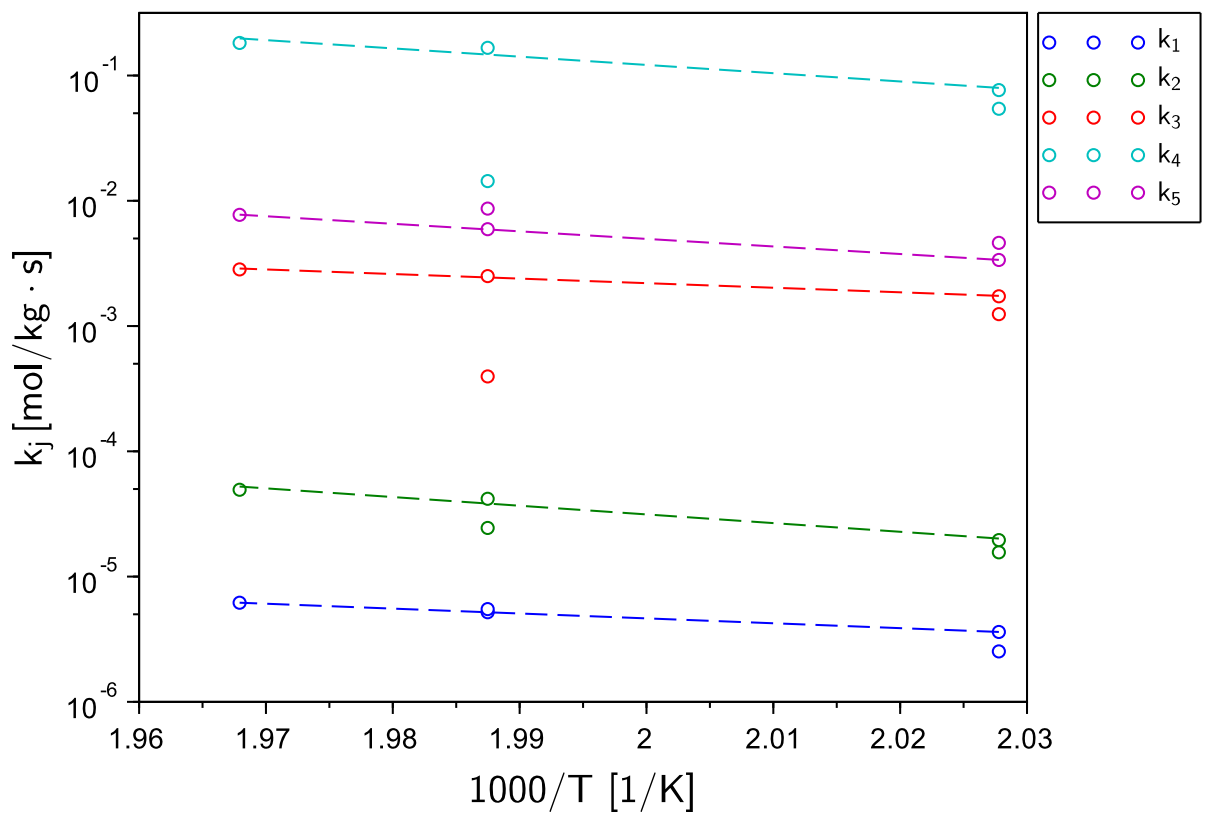

Figure 6.6: Fit of the rate constants resulting from the data of Visconti et al. (2007, 2011) to the Arrhenius expression. Dashed lines represent the model prediction

The resulting estimates of the pre-exponential factor and activation energy for $k_{1}-k_{5}$ are given in Table 6.10. For $k_{1}$ and $k_{5}$, the pre-exponential factor and activation energy are estimated with a 
high degree of confidence. This arises because of a good model prediction of the $\mathrm{CO}$ conversion data and low carbon number product quantities for the data of Visconti et al. (2007, 2011). The variability in the estimation of the pre-exponential factor and activation energy of $k_{2}-k_{4}$ is greater than that of $k_{1}$ and $k_{5}$ but can still be considered acceptable. The Arrhenius expression can thus be used to describe the temperature variation of the rate constants with some confidence.

Table 6.10: Estimated pre-exponential factors and activation energies for $k_{1}-k_{5}$

\begin{tabular}{ccc}
\hline Reaction step & $\ln \left(A_{j}\right)[\mathrm{mol} / \mathrm{kg} / \mathrm{s}]$ & $E_{a, j}[\mathrm{~kJ} / \mathrm{mol}]$ \\
\hline 1 & $5.678 \pm 0.025$ & $74.66 \pm 0.11$ \\
2 & $21.56 \pm 5.04$ & $132.7 \pm 21.0$ \\
3 & $10.67 \pm 1.42$ & $69.80 \pm 5.93$ \\
4 & $28.28 \pm 7.15$ & $126.3 \pm 29.8$ \\
5 & $22.46 \pm 0.27$ & $115.4 \pm 1.1$ \\
\hline
\end{tabular}

\subsection{Summary}

An evaluation of the predictive ability of the kinetic model developed has been performed. Emphasis was placed on the ability of the model to predict changes in experimental product distributions with changing reaction temperature in a FBR and slurry reactor. The model has been shown to effectively predict the behaviour in both these reactor types. This agrees with the findings of Visconti et al. (2016).

A good fit of the model to the product distribution data of Visconti et al. (2007, 2011), Visconti et al. (2009) and Visconti et al. (2016) was obtained. Particularly in the case of the product distribution data of Visconti et al. (2007, 2011) and Visconti et al. (2009), this was quantified by the small standard error in the estimation of $k_{1}-k_{5}$ and in the difference between the experimental and model predicted $\mathrm{CO}$ conversion. Although the model fit to the product distribution data of Visconti et al. (2016) was qualitatively good, the estimated error in the values of $k_{1}-k_{5}$ was greater relative to the other datasets. This emphasised the importance of regressing the product distribution data at a CO conversion where it is most sensitive to the model rate constants. The temperature-dependence of the rate constant could adequately be described by the Arrhenius expression.

In all cases, a vapour fraction close to unity was obtained. This indicates the appropriateness of modelling the system as a purely vapour phase reaction, a common approach in literature kinetic model development (Visconti et al., 2007, 2011; Sage et al., 2014; Todic et al., 2014; Kamal K. Pant \& Upadhyayula, 2019, Marchese et al., 2019). An advantage of the kinetic model developed in this work, however, is that the kinetic driving force is the species activity. As such, 
the magnitude of the rate constant estimates are independent of phase and will remain valid for regions of two-phase reactor operation.

The good qualitative agreement between experimental data and the model prediction highlights the strength of modelling the kinetics of FT synthesis as an equilibrium-limited process (Norval \& Phillips, 1990, Norval, 2008). This simplifies the description of the reaction without compromising on prediction quality and physical consistency. The equilibrium basis of the model thus allowed for a good description of the effect of CO conversion on product selectivity (Tucker \& van Steen, 2019). This further emphasises that the behaviour of the FT reaction system does not rely on kinetics alone (Glasser et al., 2012). 


\section{Chapter 7}

\section{Two-Phase Considerations}

\subsection{Liquid Phase Formation in Fischer-Tropsch Synthesis}

So far, it has been assumed that the reactions in FT synthesis occur only in the vapour phase. However, below $250^{\circ} \mathrm{C}$, the reaction products are partially vapour and liquid (Visconti, 2014). FT synthesis thus occurs in a three-phase system i.e. gas $\left(\mathrm{CO}, \mathrm{H}_{2}\right.$, steam and light hydrocarbon products), liquid/heavy hydrocarbon products and the solid catalyst (Sie \& Krishna, 1999). While it is often implied that the presence of a liquid phase has an influence on the reaction kinetics and hence product selectivity in FT synthesis, it does not appear to be adequately accounted for when modelling the reaction (Hildebrandt et al., 2010; Glasser et al., 2012). Furthermore, the design and performance of low temperature FT synthesis reactors is strongly dependent on the presence of a liquid phase. In slurry reactors, the presence of a liquid phase and its composition determines the reactor hydrodynamics and gas-liquid mass transfer (Visconti, 2014). In fixed-bed reactors $(F B R(s))$, the presence of a liquid phase affects heat and mass transfer effects and pressure-drop (Hildebrandt et al., 2010; Glasser et al., 2012; Visconti, 2014; Ermolaev et al., 2015). As such, it is important to consider the formation of the liquid phase during the FT reaction.

Currently, kinetic models in literature consider only a vapour phase. As such, to account for the observed characteristics of the FT product distribution (high methane yield, low ethene yield and change from mainly 1-olefins at low carbon numbers to mainly $n$-paraffins at high carbon numbers), the adsorption of 1-olefins is assumed to depend exponentially on carbon number (Sage et al., 2014; Todic et al., 2014; Marchese et al., 2019, Kamal K. Pant \& Upadhyayula, 2019). However, unless the temperature is high in the case of a reactor hotspot (FBR) or CO conversion is low (large amounts of unconverted syngas), the formation of long chain n-paraffins and 1-olefins during FT synthesis results in the catalyst pores being filled with liquid (Satterfield et al., 1985). This arises 
since the effective pressure experienced by the fluid in the catalyst pore is much higher than the system operating pressure due to capillary action (Wang et al. 2003a). The presence of a liquid phase in the catalyst pore is therefore enhanced. As such, adsorption to the active sites will occur from the liquid phase. The work of Laxmi Narasimhan et al. (2006) has shown that n-paraffin adsorption selectivity from the liquid phase is unity. The presence of a liquid phase thus eliminates the influence of adsorption on reactivity and product selectivity (Möller et al., 2009).

With an increase in carbon number of the products formed, there is a transition from the vapour to liquid phase, with hydrocarbon species of higher carbon number being more concentrated in the liquid phase. This introduces a carbon number dependence to the reaction rate in the liquid phase. For liquid-filled pores, the adsorbed or pseudo-liquid reaction phase is close to saturation (Le Grange, 2009; Möller et al., 2009). Thus, the assumption of vapour-liquid equilibrium (VLE) provides a good approximation to describe the carbon number dependence of the species reaction rates (Möller et al., 2009). This provides a practical approach to answer the following questions:

1. How much liquid forms?

2. What effect does liquid formation have on the product distribution and selectivity characteristics of FT synthesis?

3. How do the vapour phase and vapour-liquid systems compare?

\subsection{Choice of Kinetic Driving Force}

The kinetic model developed in this thesis makes use of the species activity as the kinetic driving force. The advantage of using this driving force is that it allows the influence of VLE on the kinetic behaviour and product selectivity of FT synthesis to be directly integrated (Laxmi Narasimhan et al., 2006; Mthombeni, 2009). This can be illustrated by considering the hypothetical polymerisation Reaction 7.1, where the rate of reaction is given by Equation 7.2 .

$$
A+B \rightleftharpoons A B
$$

In Equation 7.2, $a_{i}$ is the activity of species $i, k_{\text {poly }}$ is the reaction rate constant and $K^{\text {poly }}$ is the reaction equilibrium constant. The equilibrium constant is given by Equation 7.3 , where $\Delta G_{r \times n}^{\circ}$ is the Gibbs free energy of reaction at the chosen reference state. The reference state is the ideal gas state at a pressure of $1 \operatorname{bar}(a)$ and temperature of the system. This is the preferred choice in multiphase systems in the presence of supercritical species.

$$
r_{A}=k_{\text {poly }}\left(a_{A} a_{B}-\frac{a_{A B}}{K^{p o l y}}\right)
$$




$$
K^{\text {poly }}=\exp \left(-\frac{\Delta G_{r \times n}^{\circ}}{R T}\right)
$$

When the reaction is carried out purely in the vapour phase, Equation 7.2 reduces to Equation 7.4 , where $y_{i}$ and $\phi_{i}^{V}$ are the vapour phase mole fraction and fugacity coefficient of species $i$ respectively.

$$
r_{A}=k_{\text {poly }}\left(\frac{P_{\text {total }}}{P_{\text {ref }}}\right)^{2}\left(y_{A} y_{B} \phi_{A}^{V} \phi_{B}^{V}-\frac{y_{A B} \phi_{A B}^{V}}{\left(\frac{P_{\text {total }}}{P_{\text {ref }}}\right) K^{\text {poly }}}\right)
$$

When the reaction is carried out purely in the liquid phase, Equation 7.2 reduces to Equation 7.5 , where $x_{i}$ and $\phi_{i}^{L}$ are the liquid phase mole fraction and fugacity coefficient of species $i$ respectively.

$$
r_{A}=k_{\text {poly }}\left(\frac{P_{\text {total }}}{P_{\text {ref }}}\right)^{2}\left(x_{A} x_{B} \phi_{A}^{L} \phi_{B}^{L}-\frac{x_{A B} \phi_{A B}^{L}}{\left(\frac{P_{\text {total }}}{P_{\text {ref }}}\right) K^{\text {poly }}}\right)
$$

The assumption of VLE indicates that the Gibbs free energy of the system is at a minimum. Thus, the fugacity in the vapour and liquid phases are equal. The PR-EOS provides a good approximation of the VLE of the FT system (see Appendix C), resulting in the equality in Equation 7.6.

$$
\begin{array}{r}
f_{i}^{V}=f_{i}^{L} \\
y_{i} \phi_{i}^{V} P_{\text {total }}=x_{i} \phi_{i}^{L} P_{\text {total }}
\end{array}
$$

The implication of Equation 7.6 is that the rate expressions for the vapour and liquid phases are equivalent under the assumption of VLE since the driving force term is identical. Furthermore, this allows the magnitude of the rate constant to be independent of phase. The use of species activity allows the kinetic model to be capable of handling phase changes and thus, is applicable in both the vapour and liquid phases.

\subsection{Methodology}

The influence of VLE on the kinetic behaviour and product selectivity is demonstrated using a FBR. The purpose of these calculations is to illustrate the formation of liquid phase products from a purely gaseous syngas feed, producing two-phase flow at the reactor exit for a reactor type assumed to operate purely in the vapour phase (Satterfield et al., 1985). The following assumptions are made:

1. Ideal plug flow.

2. The reactor is isothermal and isobaric (Visconti et al., 2007, 2011, Kwack et al., 2011).

3. The reaction occurs at the interface between the vapour and liquid phases (Fogler, 1999). 
The vapour and liquid phases are therefore in equilibrium, which can be described by the PR-EOS.

4. Negligible internal and external mass transfer effects (Todic et al., 2014; Marchese et al., 2019).

These assumptions allow the influence of VLE on the FT product distribution and selectivity to be investigated. Equation 7.7 can thus be used, where $F_{i}$ is the molar flow rate of species $i, x$ is the normalised catalyst weight, $r_{i}$ is the rate of reaction for species $i, a_{i}$ is the species activity and $W_{\text {total }}$ is the total catalyst weight.

$$
\frac{d F_{i}}{d x}=r_{i}\left(a_{i}, k_{j}\right) W_{t o t a l}
$$

An outline of the steps used to evaluate the influence of VLE on the kinetic behaviour of FT synthesis is illustrated in Figure 7.1, adapted from Accolla (2006) and Le Grange (2009). The solution process begins by specifying the flow rate of each species in the feed as well as the temperature, pressure, $\mathrm{H}_{2} / \mathrm{CO}$ feed ratio and the rate constants of reaction step 1- 6 which give rise to typical FT reaction behaviour (high methane yield, low ethene yield and change from mainly 1-olefins at low carbon numbers to mainly n-paraffins at high carbon numbers). The numerical values of the rate constants are summarised in Table 7.1. Furthermore, the model also requires user specification of the number of phases expected to be present in the reactor to allow for an evaluation of the effect of VLE, relative to the FT reaction occurring in the vapour phase only. This determines the calculation mode of the model. A user specified value of ' 0 ' indicates that the reaction occurs in the vapour phase only, while a user specified value of ' 1 ' indicates that the reaction occurs in both the vapour and liquid phases.

Table 7.1: Rate constants for reaction step 1-6 in the single- and two-phase models

\begin{tabular}{cc}
\hline Rate constant & Value $[\mathrm{mol} / \mathrm{kg} / \mathrm{s}]$ \\
\hline$k_{1}$ & 0.0001 \\
$k_{2}$ & 0.001 \\
$k_{3}$ & 0.1 \\
$k_{4}$ & 10 \\
$k_{5}$ & 1 \\
$k_{6}$ & 0.0001 \\
\hline
\end{tabular}

The number of phases to be considered during reaction determines whether a flash calculation will be carried out at each point of the normalised catalyst weight. If the reaction occurs in the vapour phase only, then flashing is not carried out at all. Instead the activity and reaction rate are calculated based on the vapour phase only. The integration of the mole balance, Equation 7.7, is 


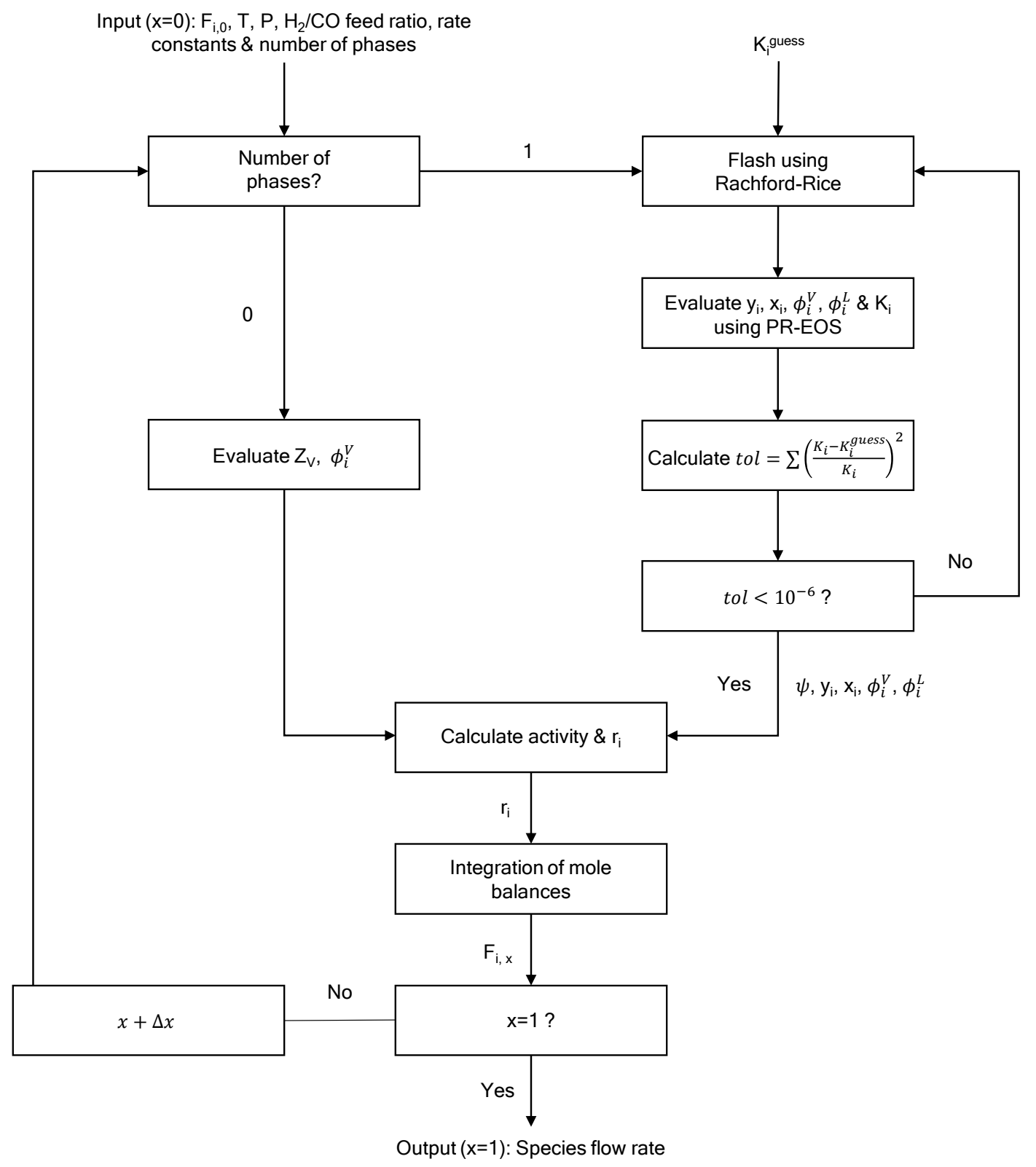

Figure 7.1: Outline of the solution process used in the two-phase FBR model 
carried out until the end point of the normalised catalyst weight is reached i.e. $x=1$.

If the reaction occurs in both the vapour and liquid phases, flashing is carried out at each point in the reactor using the Rachford-Rice algorithm. This begins by making an approximation of the separation factors $K_{i}$ using the shortcut formula, Equation 7.8, based on the Law of Corresponding States (Elliot \& Lira, 2012). The vapour and liquid phase mole fractions can then be calculated. Thereafter, the vapour and liquid phase fugacity coefficients are evaluated using the PR-EOS. The separation factor is updated as the ratio of the species liquid to vapour phase fugacity coefficients using Equation 7.9. The flash procedure converges once the relative difference between successive iterates of the estimated separation factor is below the specified tolerance.

$$
\begin{gathered}
K_{i}^{\text {guess }} \approx \frac{P_{i}^{\text {sat }}}{P_{\text {total }}}=\frac{P_{c, i} 10^{\frac{7}{3}\left(1+\omega_{i}\right)\left(1-\frac{T_{c, i}}{T}\right)}}{P_{\text {total }}} \\
K_{i}=\frac{\phi_{i}^{L}}{\phi_{i}^{V}}
\end{gathered}
$$

Thereafter, the procedure follows similarly to the vapour phase only part of the solution process. In both cases the output at the endpoint of the normalised catalyst weight $(x=1)$ is the species molar flow rate. The solution process developed can handle a transition from a vapour phase only system at the reactor entrance to a two-phase system, where both the vapour and liquid phases exist at the reactor exit.

To answer the questions posed, the model is used to evaluate the effect of VLE on the product distribution by comparing the predictions of the single- and two-phase simulations at a CO conversion of $60 \%$, feed temperature of $220^{\circ} \mathrm{C}$, pressure of $20 \mathrm{bar}(\mathrm{a})$ and $\mathrm{H}_{2} / \mathrm{CO}$ feed ratio of 2.1. This is reported to be the $\mathrm{CO}$ conversion at which the maximum $\mathrm{C}_{5+}$ selectivity is obtained in the work of Tucker \& van Steen (2019). Thereafter, the effect of VLE on the product distribution and selectivity is studied as a function of $\mathrm{CO}$ conversion at the same temperature, pressure and feed ratio. Finally, the single- and two-phase model predictions are compared to experimental data. Leckel (2005) and Leckel \& Liwanga-Ehumbu (2006) provide data of the flashed FT wax product used as a feed to the subsequent product upgrading steps through hydrocracking. This will provide insight regarding when either of the single-phase or two-phase approach will be most applicable. 


\subsection{Results and Discussion}

\subsubsection{Influence of Vapour-Liquid Equilibrium}

The influence of VLE on the product distribution of FT synthesis can be shown by comparing the single- and two-phase simulations at a CO conversion of $60 \%$. As indicated earlier, this is the $\mathrm{CO}$ conversion level at which the maximum $\mathrm{C}_{5+}$ selectivity is obtained as reported by Tucker \& van Steen (2019). For both the single- and two-phase simulations, the feed temperature, pressure and $\mathrm{H}_{2} / \mathrm{CO}$ feed ratio are $220^{\circ} \mathrm{C}, 20$ bar(a) and 2.1 respectively. These conditions are chosen to be representative of industrial FBR operation (Visconti et al., 2007, 2011). The highest carbon number $\mathrm{n}$-paraffin and 1-olefin included in each simulation is $N=81$.

In Figure 7.2, the resulting product distributions of the two simulations are compared. As shown, the influence of VLE is evident and leads to distinctive differences in the product distribution when the reaction occurs in both the vapour and liquid phases relative to when the reaction occurs only in the vapour phase. In Figure $7.2 \mathrm{a}$, the assumption of a vapour phase only reaction results in a product distribution that can be described by the ASF distribution. This indicates that the product distribution can be characterised by a single chain growth probability i.e $\alpha$. At the CO conversion of the simulation, the product is predominantly paraffinic in nature. A maximum in the total hydrocarbon distribution is reached at $C_{7}$ and $C_{8}$. Due to the series nature of the n-paraffin and 1-olefin chain growth reactions, there is a steady decrease in product quantities with increasing carbon number, which will eventually approach zero.

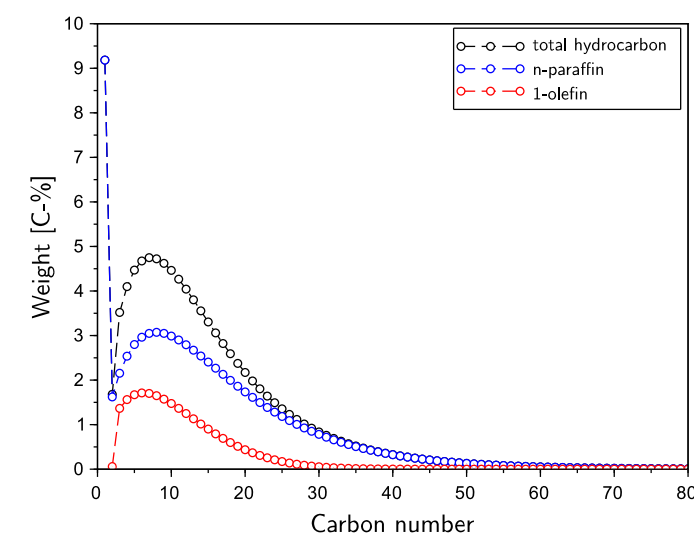

(a) Reaction in the vapour phase only

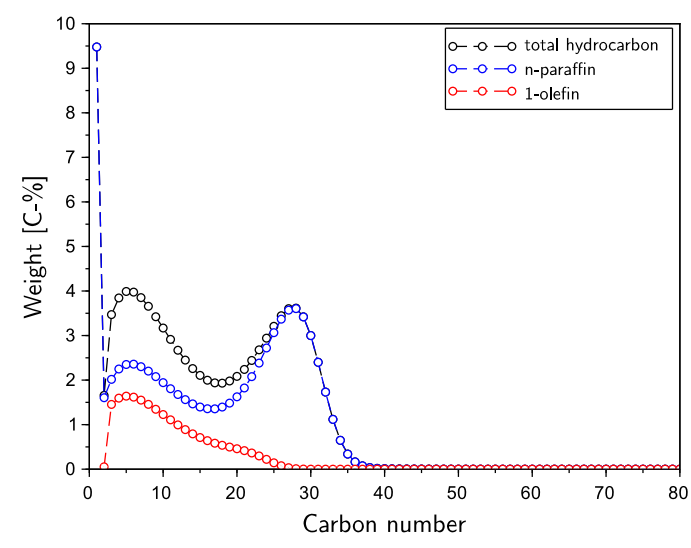

(b) Reaction in both the vapour and liquid phase

Figure 7.2: Comparison of the vapour phase and vapour-liquid product distributions at a $\mathrm{CO}$ conversion of $60 \%, 220^{\circ} \mathrm{C}, 20 \mathrm{bar}(\mathrm{a})$ and $\mathrm{H}_{2} / \mathrm{CO}$ feed ratio of 2.1 
In contrast in Figure 7.2b, when the reaction occurs in both the vapour and liquid phases, two maxima occur in the total hydrocarbon and n-paraffin product distributions respectively. Initially, the product distribution reaches a maximum at $C_{5}$ and $C_{6}$ before steadily decreasing until $C_{17}$ and $\mathrm{C}_{18}$. However, unlike the single-phase simulation in Figure $7.2 \mathrm{a}$, a rise in product weight fractions occurs to reach a second maximum at $\mathrm{C}_{29}$. At the $\mathrm{CO}$ conversion of the simulation, the product is predominantly paraffinic as in Figure $7.2 \mathrm{a}$. As such, this maximum is produced by the n-paraffin product distribution. The behaviour in Figure $7.2 \mathrm{~b}$ indicates that the product distribution can be characterised by two chain growth probabilities i.e. two $\alpha$ theory (Patzlaff et al., 1999).

The behaviour in Figure $7.2 \mathrm{~b}$ arises as a result of a transition from the vapour phase to liquid phase with increasing carbon number. This is illustrated by the partition curve between the vapour and liquid phases in Figure 7.3a, which is produced by the VLE calculation. With increasing carbon number, there is an increase in boiling point. As such species of low carbon number $\left(C_{1}-C_{10}\right)$ have a low boiling point and thus will exist in the vapour phase. In contrast, species of high carbon number ( $\mathrm{C}_{30}$ and greater) have a much higher boiling point and thus will exist in the liquid phase at the conditions of the simulation. Species of intermediate carbon number between these two bounds will distribute between the vapour and liquid phases. This has also been demonstrated in the work of Visconti (2014). Thus, product species will exist in the phase which ensures that the Gibbs free energy of the system is at a minimum, the criterion for equilibrium.

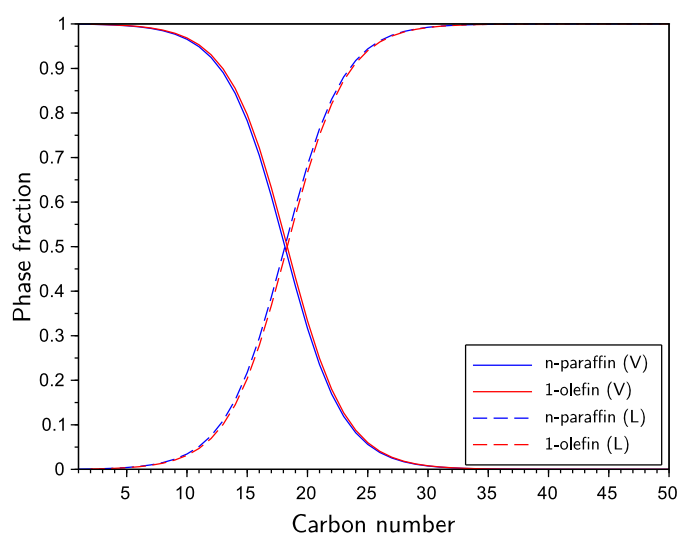

(a) Phase fraction as a function of carbon number

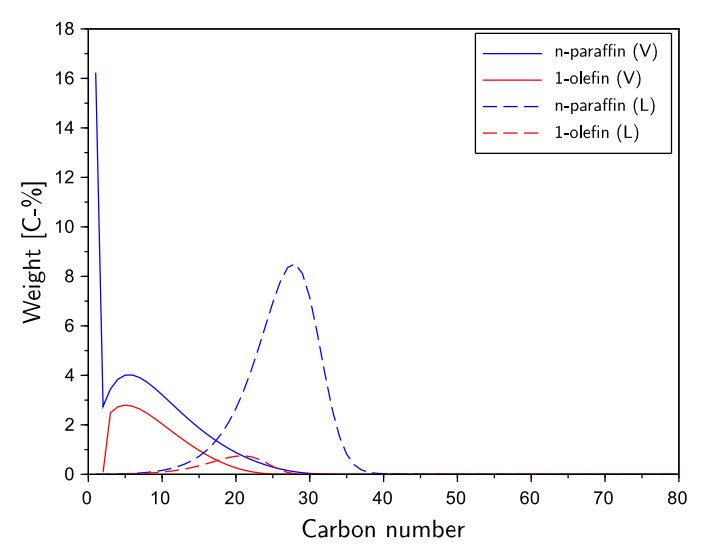

(b) Vapour and liquid phase product distributions

Figure 7.3: Influence of VLE on the FT product distribution at a $\mathrm{CO}$ conversion of $60 \%, 220^{\circ} \mathrm{C}$, $20 \operatorname{bar}(\mathrm{a})$ and $\mathrm{H}_{2} / \mathrm{CO}$ feed ratio of 2.1

The profiles in Figure $7.2 \mathrm{~b}$ can therefore be interpreted as the vapour and liquid product distributions being superimposed on one another. This becomes clearer when Figure $7.3 \mathrm{~b}$ is studied. In Figure $7.3 \mathrm{~b}$, both the vapour phase $\mathrm{n}$-paraffin and 1-olefin product distributions consist mainly of species 
of low carbon number, which approaches zero around $C_{30}$. This is the carbon number at which the liquid phase dominates in Figure 7.3a. In contrast, the liquid phase n-paraffin and 1-olefin product distributions begin at a carbon number of $C_{10}$. This is the carbon number from which the transition into the liquid phase begins. The two maxima that arise in the product distribution in Figure $7.2 \mathrm{~b}$ thus correspond to the vapour and liquid phase respectively. This reiterates the basis for two separate $\alpha$ values being needed to characterise the product distribution in Figure $7.2 \mathrm{~b}$

The bell-shape of the liquid phase product distribution in Figure $7.3 \mathrm{~b}$ was also observed in the characterisation of Sasol-Schumann type 4110 FT wax by van der Laan (1999). The main constituent of this FT wax is reported by van der Laan (1999) to be n-paraffins of carbon number between $\mathrm{C}_{21}-\mathrm{C}_{36}$, with a maximum at $\mathrm{C}_{28}$. Similar behaviour was found in Figure $7.3 \mathrm{~b}$, demonstrating that the trend is physically consistent.

As indicated and observed in Figure $7.3 \mathrm{a}$, the transition into the liquid phase begins at $\mathrm{C}_{10}$, with the liquid phase dominating from $\mathrm{C}_{17}$. This is the point at which a local minimum occurs in Figure $7.2 \mathrm{~b}$ and is the carbon number from which the liquid phase begins to dominate. The condensation of n-paraffins of carbon number from $C_{10}$ drives the forward n-paraffin chain growth reaction. This is achieved by a reduction in the vapour phase concentration of $n$-paraffins of carbon number $\mathrm{C}_{10}$ and greater relative to the vapour phase only simulation. This leads to the depression in the product distribution for which vapour phase products are favoured but facilitates the rise in the product distribution for which liquid phase products are favoured in Figure $7.2 \mathrm{~b}$

The vapour phase only simulation in Figure $7.2 \mathrm{a}$ results in much greater quantities of products of carbon number $\mathrm{C}_{35}$ onwards than the simulation in which both vapour and liquid phases are considered in Figure 7.2b. Based on the partition curve in Figure 7.3a, species of $C_{35}$ onwards are all liquid. These species are concentrated in the liquid phase and thus the reaction of species for these carbon numbers is driven by the liquid phase. Consequently, their high concentration in the liquid phase favours the reverse chain growth reactions. This leads to the second local maximum being established in Figure $7.2 \mathrm{~b}$.

The coupling of VLE to the kinetics thus drives and favours the formation of a liquid phase. Despite the formation of a liquid phase, at the CO conversion of the two-phase simulation the total molar vapour fraction is $0.9939^{1}$. This is however enough to lead to distinct differences in the resulting product distribution. This emphasises the importance of considering liquid phase formation in FT synthesis, even in a predominantly vapour system (Satterfield et al., 1985). The analyses shown should however be extended to investigate the influence of VLE on the product distribution and

\footnotetext{
${ }^{1}$ Total vapour fraction on a mass basis is 0.9251 . This difference arises since the heavier hydrocarbons exist in the liquid phase.
} 
product selectivity over the entire CO conversion range. This is the subject of the next subsection.

\subsubsection{Effect of CO Conversion on the Single- and Two-phase Simulations}

The effect of VLE on the kinetic behaviour of FT synthesis needs to be evaluated over the entire $\mathrm{CO}$ conversion range to provide insight regarding the amount of liquid products which form during reaction. This allows for an evaluation regarding the effect of liquid formation on product selectivity as a function of $\mathrm{CO}$ conversion. Ultimately, this will provide an indication of where it is best to operate the reactor to favour selectivity to the desired products.

Although it is customary in work on $F T$ synthesis to lump selectivity to $C_{1}, C_{2}, C_{3}, C_{4}, C_{5+}$ and $\mathrm{CO}_{2}$, use of these norms lacks the necessary detail to allow for meaningful comparison between the single- and two phase simulations (see Appendix C). It is more informative to use the definition and conventions of the different fuel types obtained from crude oil refinery processes (Kroschwitz \& Mark, 1996). The lumped product grades used to compare the single- and two-phase simulations are summarised in Table 7.2 .

Table 7.2: Definition of the different product grades obtained from crude oil refinery processes

\begin{tabular}{lc}
\hline Name & Components \\
\hline Methane & $\mathrm{C}_{1}$ \\
Liquified petroleum gas (LPG) & $\mathrm{C}_{2}-\mathrm{C}_{4}$ \\
Gasoline & $\mathrm{C}_{5}-\mathrm{C}_{9}$ \\
Diesel & $\mathrm{C}_{10}-\mathrm{C}_{20}$ \\
Wax & $\mathrm{C}_{21+}$ \\
\hline
\end{tabular}

The selectivity to the product grades in Table 7.2, which are lumped by carbon number, are calculated on a carbon basis for LPG, gasoline and diesel using Equation 7.10, In Equation 7.10, $n_{\text {lower }}$ is the lower bound carbon number in the product grade e.g. $C_{2}$ for LPG, $n_{\text {upper }}$ is the upper bound carbon number in the product grade e.g. $C_{4}$ for LPG, $n_{i}$ is the number of carbon atoms in the product, $F_{C_{n_{i}} H_{2 n_{i}+2}}$ and $F_{C_{n_{i}} H_{2 n_{i}}}$ are the flow rates of n-paraffin and 1-olefin products, $F_{C O, 0}$ is the feed flow rate of $\mathrm{CO}$ and $X_{C O}$ is the $\mathrm{CO}$ conversion. The selectivity to wax can then be calculated using Equation 7.11 to ensure that the product selectivity is normalised.

$$
\begin{gathered}
S_{\text {Product grade }}=\frac{\sum_{i=n_{\text {lower }}}^{n_{\text {upper }}} n_{i}\left(F_{C_{n_{i}} H_{2 n_{i}+2}}+F_{C_{n_{i}} H_{2 n_{i}}}\right)}{F_{C O, 0} X_{C O}} \times 100 \\
S_{\text {Wax }}=100-\left(S_{\text {Methane }}+S_{L P G}+S_{\text {Gasoline }}+S_{\text {Diesel }}+S_{C O 2}\right)
\end{gathered}
$$

In Figure 7.4, the product selectivity as a function of $\mathrm{CO}$ conversion is shown for the single- and 
two-phase simulations. For the single-phase simulation, Figure 7.4a, at $\mathrm{CO}$ conversions below 5\%, the primary selectivity is to methane and LPG. This arises because $\mathrm{CH}_{2}$ coupling to ethene and $\mathrm{CH}_{2}$ hydrogenation to methane are the main reactions occurring, with a low rate of $n$-paraffin and 1-olefin chain growth. Thereafter, the selectivity shifts to gasoline with increasing CO conversion and hence chain growth. A maximum gasoline selectivity is obtained at a $\mathrm{CO}$ conversion of $5 \%$. Above a CO conversion of $15 \%$, the main product grade that forms is diesel. A maximum diesel selectivity of $35 \mathrm{C}-\%$ is established at a CO conversion of $60 \%$. Across the entire CO conversion range, the single-phase simulation predicts a much lower selectivity to wax relative to diesel.

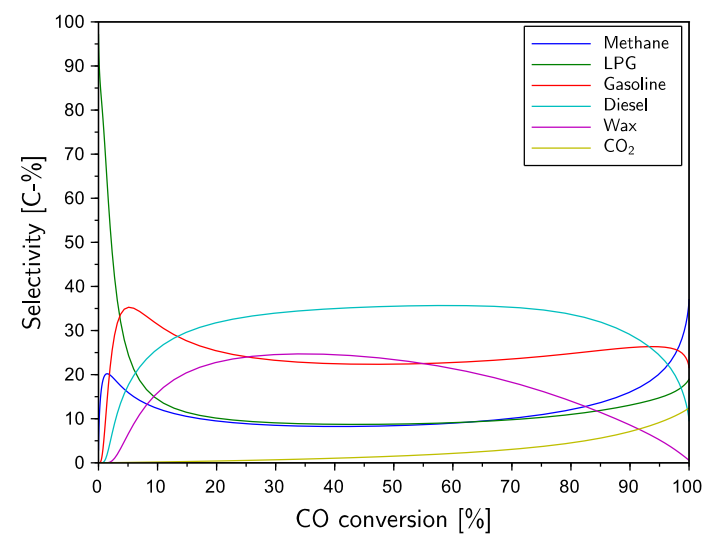

(a) Reaction occurs in the vapour phase only

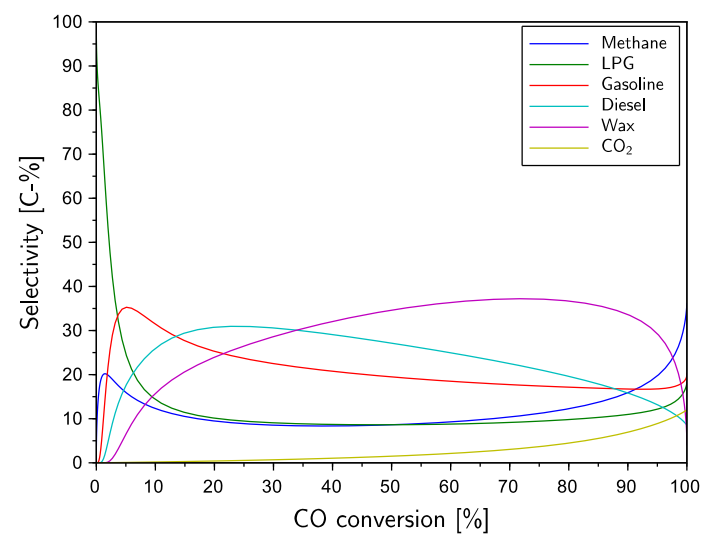

(b) Reaction occurs in the vapour and liquid phases

Figure 7.4: Influence of VLE on the product selectivity as a function of $\mathrm{CO}$ conversion at $220^{\circ} \mathrm{C}$, $20 \operatorname{bar}(\mathrm{a})$ and $\mathrm{H}_{2} / \mathrm{CO}$ feed ratio of 2.1

Once the maximum diesel selectivity has been reached, a rise in methane, LPG and gasoline selectivity is observed. This arises due to the reverse chain growth reactions being favoured, leading to a shift in selectivity to favour low carbon number products. Above a CO conversion of $95 \%$, the gasoline selectivity declines, with a much steeper rise in methane selectivity. This occurs due to the large thermodynamic driving force to form methane as the system approaches complete chemical equilibrium.

The two-phase simulation in Figure $7.4 \mathrm{~b}$ produces the same trend as the single-phase simulation for the selectivity to all product grades until a CO conversion of $20 \%$. Above a CO conversion of $20 \%$ and in contrast to the single-phase simulation, the selectivity to wax increases until a CO conversion of $75 \%$ resulting in a decline in the selectivity to gasoline and diesel. The two-phase simulation predicts a much greater selectivity to wax relative to diesel. Once the maximum wax selectivity is obtained, a decline in wax selectivity leads to a rise in methane, gasoline and LPG selectivity. This indicates that the reverse chain growth reactions are favoured, facilitating the 
selectivity back to lower carbon number products. This ultimately will lead to the methane being the dominant hydrocarbon product at complete chemical equilibrium in the same way as in the single-phase simulation.

The difference in selectivity predictions in Figure $7.4 \mathrm{a}$ and $7.4 \mathrm{~b}$ over the simulated $\mathrm{CO}$ conversion range is attributed to the formation of liquid. This can be explained by considering the vapour fraction profile in the two-phase simulation as a function of $\mathrm{CO}$ conversion in Figure 7.5. In Figure 7.5. up to a CO conversion of $20 \%$, the vapour fraction is above or equal to 0.999 . Consequently, the reaction occurs mainly in the vapour phase. This leads to the same trends in the selectivity profiles being obtained in the single- and two-phase simulations up to a CO conversion of $20 \%$.

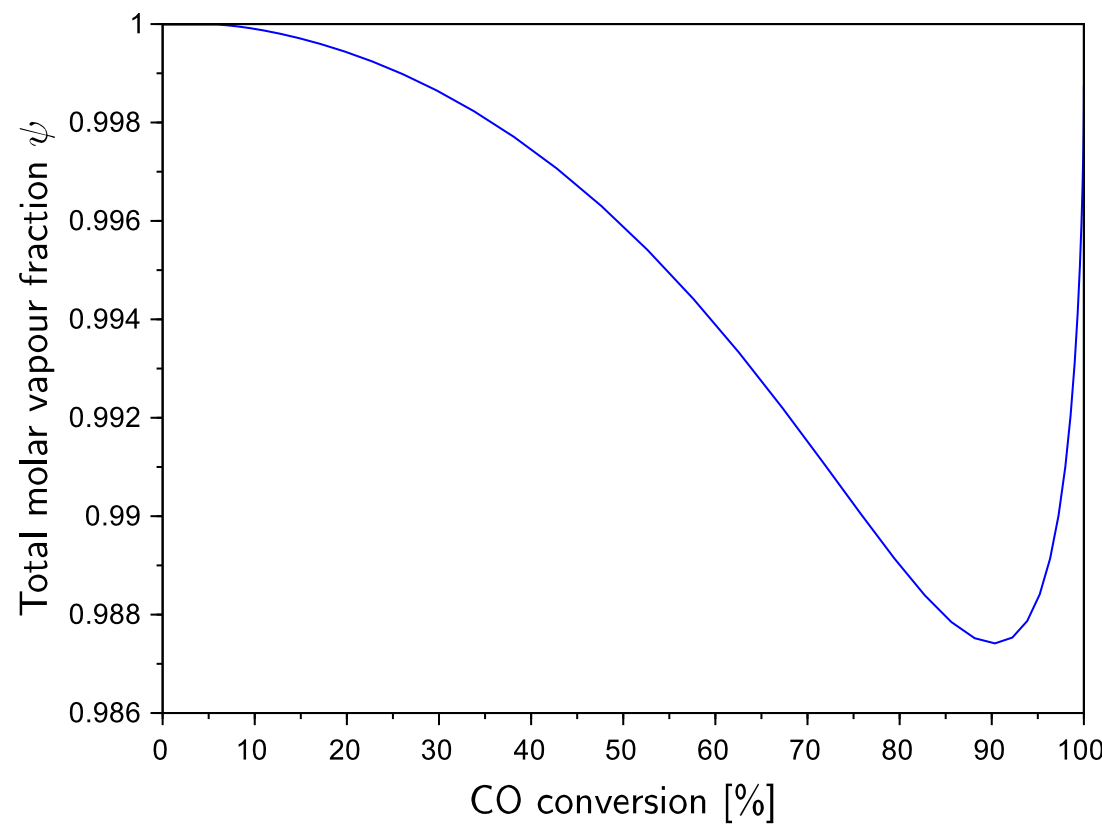

Figure 7.5: Vapour fraction as a function of $\mathrm{CO}$ conversion at $220^{\circ} \mathrm{C}, 20 \mathrm{bar}(\mathrm{a})$ and $\mathrm{H}_{2} / \mathrm{CO}$ feed ratio of 2.1

With increasing CO conversion and hence liquid formation, the selectivity to wax becomes more favourable. This arises because of the condensation of species of carbon number $\mathrm{C}_{21+}$ which drives the forward chain growth reactions. This favours the selectivity to wax as species in the gasoline and diesel product grades are more reactive towards chain growth, leading to a decrease in selectivity in these product grades until a CO conversion of $75 \%$. Thus, liquid formation favours the production of mainly high molecular weight waxes, the reported main product of FT synthesis (Espinoza et al., 1999). 
The vapour fraction profile in Figure 7.5 indicates that while the reaction system is mainly in the vapour phase, liquid formation although low, contributes to significant differences in the calculated product selectivity profiles in Figure $7.4 \mathrm{a}$ and $7.4 \mathrm{~b}$. This is especially evident for $\mathrm{CO}$ conversions between $20-90 \%$. The operation of the reactor in this CO conversion regimes means that the inclusion of a VLE calculation in the reactor is essential to provide a prediction of liquid formation and hence product selectivity. For CO conversions below 20\%, the single- and two-phase simulations produce the same trends. This indicates that assuming the reaction occurs in the vapour phase only in this $\mathrm{CO}$ conversion regime will provide a good prediction of product selectivity and reduce the calculation effort. The minimum vapour fraction in Figure 7.5 occurs at a $\mathrm{CO}$ conversion of $90 \%$, which corresponds to the maximum wax yield of $37.75 \mathrm{C}-\%$. Once this maximum is established, the vapour fraction rises as the system approaches a thermodynamically favoured methane product at complete chemical equilibrium, where the vapour phase is favoured.

It is known that lumping can distort kinetic behaviour (Möller et al., 2009). Thus, the influence of VLE on the total hydrocarbon, n-paraffin and 1-olefin product distributions, as a function of CO conversion, is also studied. The resulting product distributions are shown in Figure 7.6 at a CO conversion of $20 \%, 40 \%, 60 \%, 80 \%$ and $95 \%$. The reaction temperature, pressure and $\mathrm{H}_{2} / \mathrm{CO}$ feed ratio are $220^{\circ} \mathrm{C}, 20$ bar(a) and 2.1 respectively.

When the reaction occurs in the vapour phase only, there is initially a shift in the product distribution from products of low carbon number to products of high carbon number for $\mathrm{CO}$ conversions between $20-60 \%$ in Figure $7.6 \mathrm{a}$. This arises as a result of an increase in the extent of the chain growth reactions. However, with increases in CO conversion from 60-95\%, the total hydrocarbon product distribution shifts to containing products of mainly low carbon number. This is seen as the rise in the product distribution curves between $\mathrm{C}_{2}-\mathrm{C}_{10}$ in Figure $7.6 \mathrm{a}$. This facilitates the rise in methane selectivity observed. The n-paraffin product distribution in Figure 7.7 a corresponds closely to the total hydrocarbon product distribution in Figure $7.6 \mathrm{a}$ indicating that the product is mainly paraffinic in nature at the $\mathrm{CO}$ conversions simulated.

When the reaction occurs in both the vapour and liquid phases, two maxima are observed for all $\mathrm{CO}$ conversions in Figure 7.6b. The first peak corresponds to products in the vapour phase while the second peak corresponds to products in the liquid phase. With an increase in CO conversion, the peak corresponding to the vapour phase steadily decreases. This results in a rise in the peak corresponding to the liquid phase until a CO conversion of $80 \%$. This corresponds to the rise in wax selectivity observed in Figure 7.4b. Like the single-phase simulation, the n-paraffin product distribution in Figure $7.7 \mathrm{~b}$ corresponds closely to the total hydrocarbon product distribution in Figure $7.6 \mathrm{~b}$. This indicates that the product is highly paraffinic in nature. For the two-phase simulation, the quantity of products of carbon number $\mathrm{C}_{35}$ and greater is lower than predicted by 
the single-phase simulation.

The difference in the single- and two-phase product distributions arises due to the differences in reactivity when the reaction occurs in the vapour only compared to when the reaction occurs in both the vapour and liquid phases. The partition curve in Figure $7.3 \mathrm{a}$ indicates that there is a transition from the vapour phase to liquid phase from $\mathrm{C}_{10}$. The condensation of species of carbon number $C_{10}$ thus drives the forward chain growth reactions, favouring the formation of species of carbon number $\mathrm{C}_{21+}$. This continues as more liquid forms with increasing $\mathrm{CO}$ conversion.

The two-phase simulation predicts a lower formation of products of carbon number $C_{35}$ and greater. In the single-phase simulation, the forward chain growth reaction is always favoured due to low product concentrations of products of carbon number $\mathrm{C}_{35}$ and greater in the vapour phase. However, in the two-phase simulation, products of carbon number $\mathrm{C}_{35}$ and greater are all liquid and thus concentrated in the liquid phase. This favours the reverse chain growth reactions leading to the second local maximum in the product distribution being established. Consequently, species of carbon number $\mathrm{C}_{35}$ and greater are preferentially consumed instead of formed. This leads to lower predicted quantities of carbon number $\mathrm{C}_{35}$ and greater in the two-phase simulation than in the single-phase simulation for all CO conversions in Figure 7.6 .

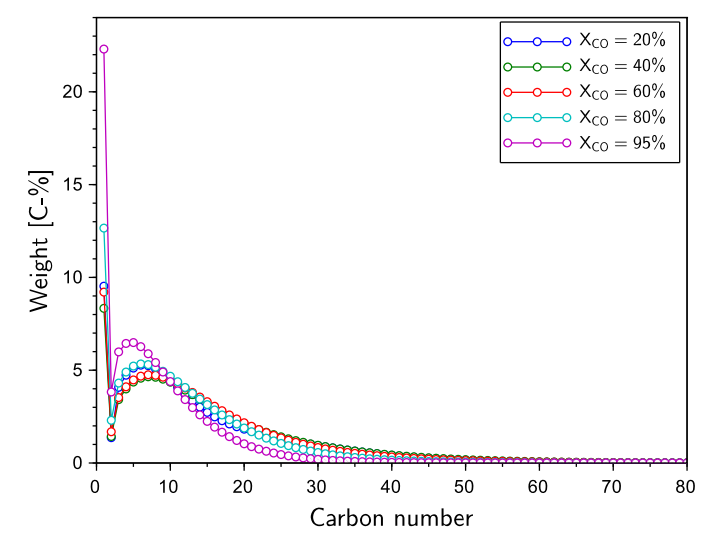

(a) Reaction in vapour phase only

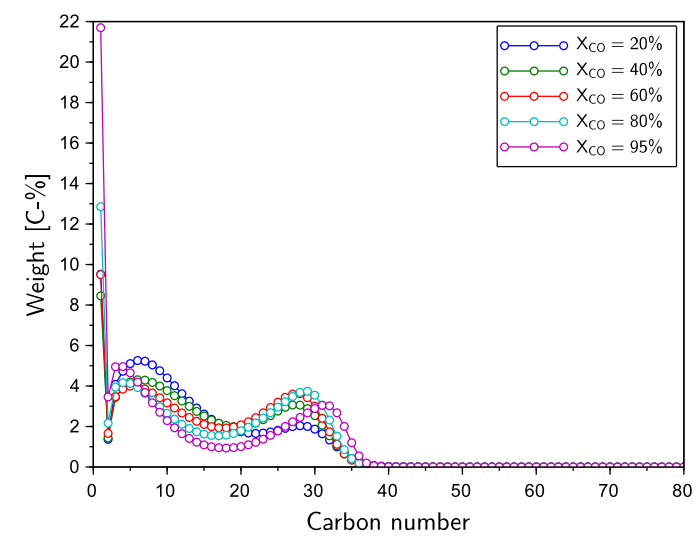

(b) Reaction in vapour and liquid phase

Figure 7.6: Comparison of the total hydrocarbon product distributions as a function of $\mathrm{CO}$ conversion at $220^{\circ} \mathrm{C}, 20$ bar(a) and $\mathrm{H}_{2} / \mathrm{CO}$ feed ratio of 2.1 


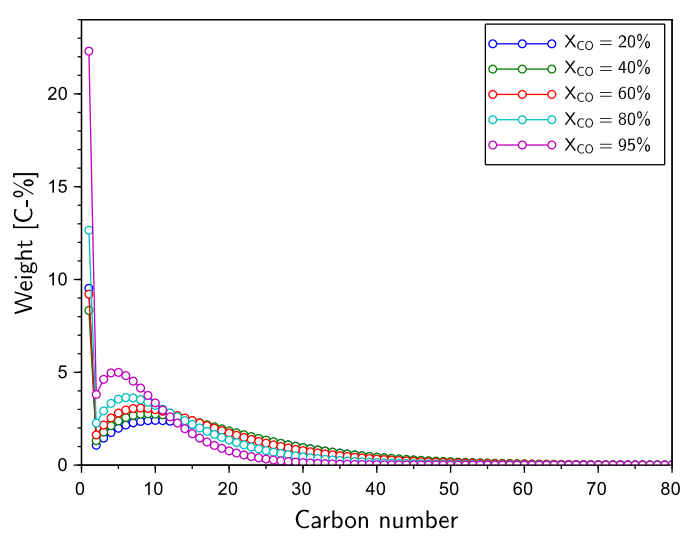

(a) n-paraffin: vapour phase only

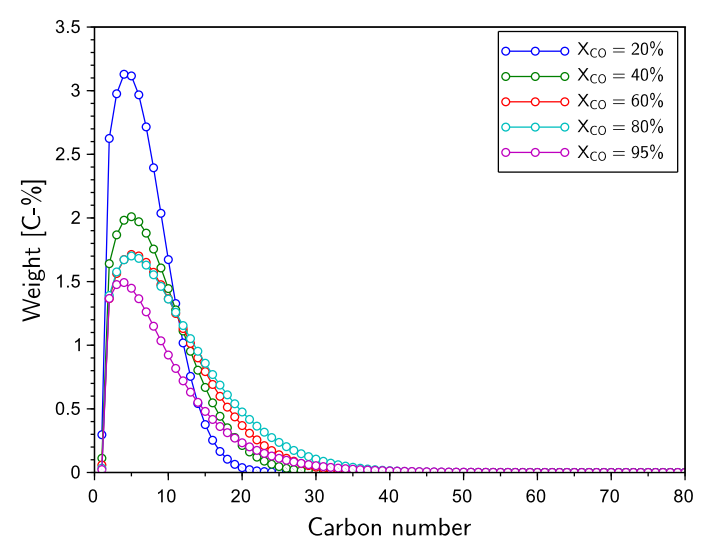

(c) 1-olefin: vapour phase only

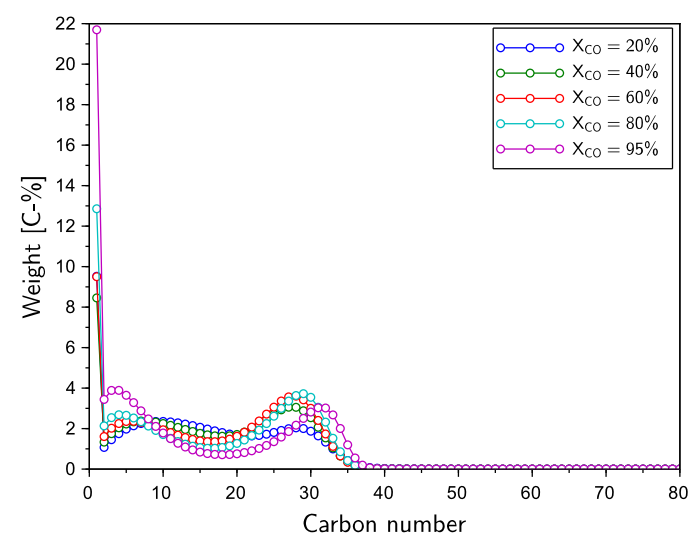

(b) n-paraffin: vapour and liquid phase

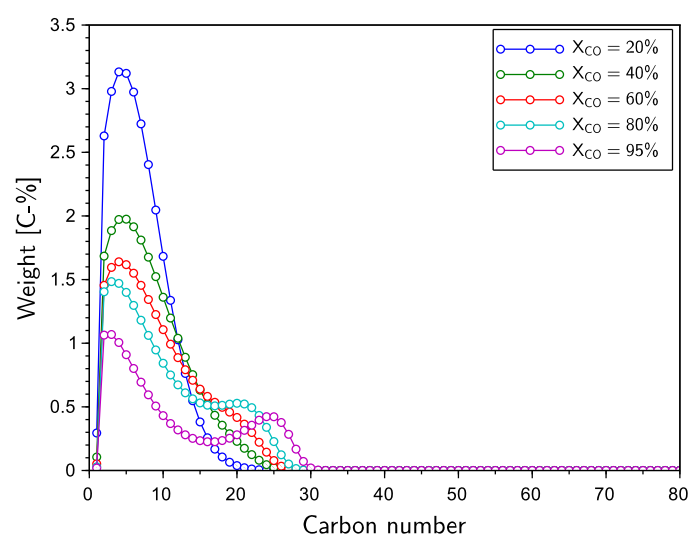

(d) 1-olefin: vapour and liquid phase

Figure 7.7: Comparison of the n-paraffin and 1-olefin product distributions as a function of $\mathrm{CO}$ conversion at $220^{\circ} \mathrm{C}, 20 \mathrm{bar}(\mathrm{a})$ and $\mathrm{H}_{2} / \mathrm{CO}$ feed ratio of 2.1 


\subsection{Comparison to Experimental Data}

The objective of this section is to provide insight regarding whether the single-phase or two-phase simulation is more appropriate in describing the kinetic behaviour of FT synthesis. The main difference between the single-phase and two-phase simulations is the resulting product distribution, specifically for products in the liquid phase i.e. the wax products. Thus, the predicted product distribution from the single- and two-phase simulations were compared to the wax product from industrial LTFT reactors, the feed to the product upgrading step through hydrocracking. This will provide an increased understanding regarding the operation of industrial LTFT reactors and the conditions under which a single-phase or two-phase modelling approach will be required.

The work of Leckel (2005) provides data regarding the wax product from iron-catalysed FT synthesis in Sasol LTFT reactors, namely the ARGE (Arbeitsgemeinschaft Ruhrgebiet) tubular and slurry reactors. No detail was provided regarding the operating conditions under which the wax products from these two reactors were formed. The feed conditions (temperature, pressure and $\mathrm{H}_{2} / \mathrm{CO}$ feed ratio) were thus adjusted until the model output yielded a similar shape to the reported distribution of the wax product. Interestingly, only minor adjustments were required to be made to the model rate constants corresponding to reaction steps 2, 3 and 4 respectively. These rate constants were adjusted since the reactor product is reported to have an n-paraffin content of about $94 \mathrm{wt} . \%$ Leckel (2005).

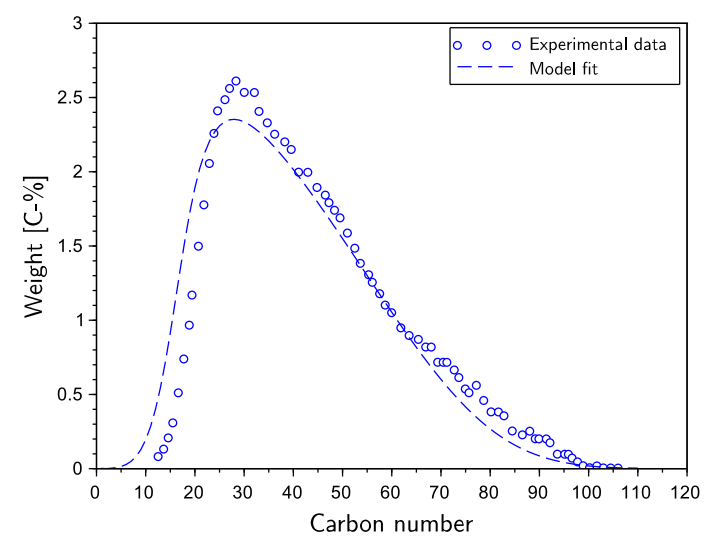

(a) ARGE tubular reactor wax product

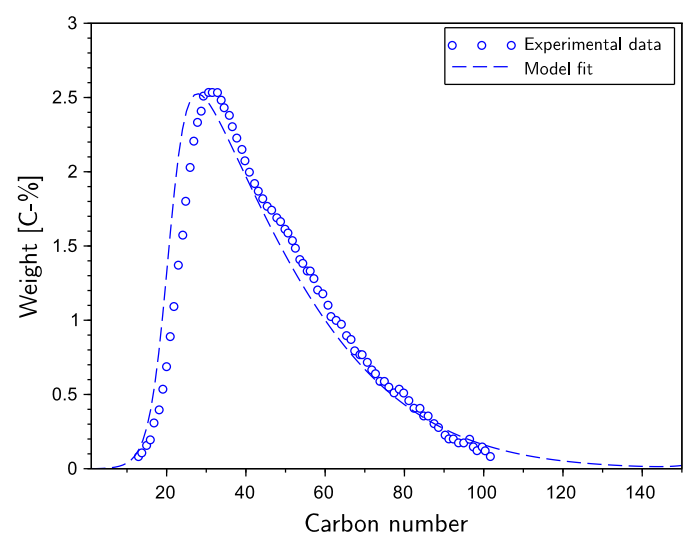

(b) Slurry reactor wax product

Figure 7.8: Model comparison to the wax product from Sasol LTFT reactors (Leckel, 2005)

The model fit to the data of Leckel (2005) is shown in Figure 7.8. A qualitatively good fit to the ARGE tubular reactor wax product in Figure $7.8 \mathrm{a}$ is achieved assuming isothermal single-phase simulation. The temperature and pressure are $220^{\circ} \mathrm{C}$ and 35 bar(a) respectively, at an $\mathrm{H}_{2} / \mathrm{CO}$ feed 
ratio of 2 . The CO conversion is $39.46 \%$, with the model rate constants summarised in Table 7.3 . These conditions lie within the range of the reported operating conditions of the ARGE tubular reactor by Espinoza et al. (1999). Although the operating CO conversion and pressure suggest that two phases should be present in the reactor, it should be noted that, the ARGE tubular reactor does not operate isothermally industrially due to the high exothermicity of the FT reactions (Espinoza et al., 1999; Sie \& Krishna, 1999). A rise in temperature due to reaction thus favours reactor operation in the vapour phase. This could lead to a single-phase simulation being more appropriate to predict the wax product.

In Figure 7.8b, a qualitatively good fit to the wax product distribution from the slurry reactor is also achieved using a single-phase simulation. The operating conditions are $220^{\circ} \mathrm{C}$ and $28 \mathrm{bar}(\mathrm{a})$ with an $\mathrm{H}_{2} / \mathrm{CO}$ feed ratio of 2 . The $\mathrm{CO}$ conversion is $20.63 \%$, with the model rate constants summarised in Table 7.3. These conditions correspond to the reported operating conditions at which Fe-based catalysts are most productive in FT synthesis (Espinoza et al., 1999). The low CO conversion lies within the operating regime in which the reaction occurs mainly in the vapour phase. Consequently, this leads to a single-phase simulation being more appropriate to predict the wax product.

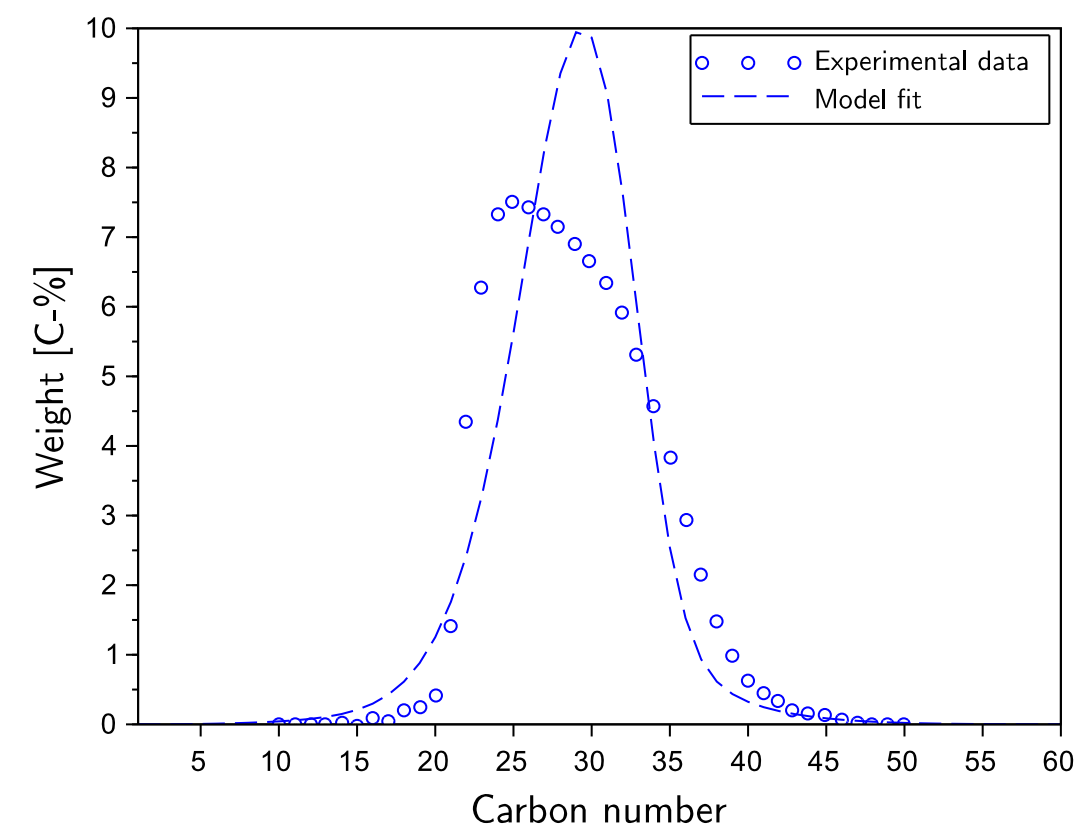

Figure 7.9: Model comparison to Sasol LTFT M5 wax (Leckel \& Liwanga-Ehumbu, 2006)

The work of Leckel \& Liwanga-Ehumbu (2006) investigated the hydrocracking of Sasol M5 wax, 
a wax fraction obtained following distillation and hydrotreating at Sasol Waxes. A qualitatively good fit to the product distribution could be obtained using a two-phase simulation in a FBR. In Figure 7.9, the wax product distribution is bell-shaped, consisting of products of carbon numbers between $\mathrm{C}_{10}-\mathrm{C}_{50}$. The operating conditions are $215^{\circ} \mathrm{C}$ and $20 \mathrm{bar}(\mathrm{a})$ with an $\mathrm{H}_{2} / \mathrm{CO}$ feed ratio of 2.1 . The model predicted CO conversion is $11.25 \%$. The model shows a qualitatively good fit to the tail ends of distribution, differing in its prediction of the peak. This is the possible result of assuming VLE, which might not be guaranteed during distillation. Nonetheless, the prediction is within an order of magnitude. It is difficult to judge whether the qualitatively good description of the product distribution arises because of the additional processing steps involved to produce the Sasol M5 wax fraction.

From Table 7.3 , the rate constants that result when the simulated wax product is compared to the wax product from Sasol processes are all within the same order of magnitude. The rate constant values differ only slightly in the case of the ARGE tubular and slurry reactors from the values given in Table 7.1. This indicates that the magnitude of the rate constants in Table 7.1 serves as a good starting point to represent the product from different reactors. It also emphasises that the shape of the product distribution is determined thermodynamically by the value of equilibrium constants rather than kinetically through the value of the rate constants alone. In the case of the model fit to the Sasol M5 wax, the shape of the distribution is determined by the effect of VLE on the reaction kinetics.

Table 7.3: Model rate constants when compared to the wax product from Sasol processes

\begin{tabular}{|c|c|c|c|}
\hline & $\begin{array}{l}\text { ARGE tubular reactor } \\
\text { (Leckel, 2005) }\end{array}$ & $\begin{array}{l}\text { Slurry reactor } \\
(\text { (Leckel, 2005) }\end{array}$ & $\begin{array}{l}\text { Sasol M5 Wax } \\
(\text { (Leckel \& Liwanga- } \\
\text { Ehumbu, 2006) }\end{array}$ \\
\hline Rate constant & Value $[\mathrm{mol} / \mathrm{kg} / \mathrm{s}]$ & Value $[\mathrm{mol} / \mathrm{kg} / \mathrm{s}]$ & Value $[\mathrm{mol} / \mathrm{kg} / \mathrm{s}]$ \\
\hline $\mathrm{k}_{1}$ & 0.0001 & 0.0001 & 0.0001 \\
\hline$k_{2}$ & 0.0008 & 0.001 & 0.001 \\
\hline$k_{3}$ & 0.11 & 0.2 & 0.1 \\
\hline $\mathrm{k}_{4}$ & 11 & 9 & 10 \\
\hline$k_{5}$ & 1 & 1 & 1 \\
\hline$k_{6}$ & 0.0001 & 0.0001 & 0.0001 \\
\hline
\end{tabular}

Generally, it was found that the wax product could be represented by a single-phase simulation for iron-catalysed FT synthesis. The contributing factors to this choice relate to the operating characteristics of the reactor e.g. temperature rise in the case of a FBR, operating conditions which maximise catalyst productivity e.g. low CO conversion and the highest carbon number product present in the wax product distribution. Both the single- and two-phase simulations are therefore powerful tools to describe wax product distributions obtained from LTFT reactors. 


\subsection{Summary}

Liquid formation in FT synthesis has been shown to affect the product distribution and product selectivity. This effect could be quantified by simultaneous VLE and kinetic calculations. To effectively incorporate the effect of VLE on the kinetic behaviour of FT synthesis, the kinetic driving force chosen was the species activity. This allowed the same kinetic model to be applied across both the vapour and liquid phases with the magnitude of the rate constants unaffected by phase changes.

Even for a system in which the vapour phase dominates, a small amount of liquid was shown to alter product selectivity. There is a transition from the vapour to liquid phase from $\mathrm{C}_{10}$ at the conditions of FT synthesis. Single-phase simulations are valid for CO conversions up to $20 \%$. Between CO conversions of $20-90 \%$, it becomes essential to incorporate a VLE calculation. Singlephase simulations predict that $\mathrm{FT}$ synthesis is mainly selective towards products of carbon number in the diesel product grade. More correctly, two-phase simulations predict that FT synthesis is mainly selective towards products of carbon number in the wax product grade. A maximum wax yield of $37.75 \mathrm{C}-\%$ is achieved at a CO conversion of $90 \%, 220^{\circ} \mathrm{C}, 20$ bar(a) and $\mathrm{H}_{2} / \mathrm{CO}$ feed ratio of 2.1. A maximum in the wax yield corresponds to a minimum vapour fraction. Above a CO conversion of $90 \%$, both the single- and two-phase simulations favour the formation of methane, the most thermodynamically favoured hydrocarbon product.

The effect of a VLE calculation on FT kinetic behaviour results in clear differences between the single- and two-phase product distributions. The formation of liquid favours the reaction of products of carbon number $C_{10}-C_{17}$ towards products of carbon number $C_{18}-C_{29}$. Consequently, two maxima are observed in the product distribution from the two-phase simulation, corresponding to the vapour and liquid phases respectively. This, however, results in much lower quantities of products of carbon number $\mathrm{C}_{35}$ onwards in the two-phase simulation relative to the single-phase simulation.

Comparisons were made between the wax product obtained from Sasol processes to the wax product obtained from the single- and two-phase simulations. For iron-catalysed FT synthesis, the singlephase simulations were shown to give a good prediction of the wax product from ARGE tubular and slurry reactors. Generally, these wax products contained species of carbon number $C_{20}-C_{110+}$ which is best described by vapour phase reactor operation. This may be a consequence of the operating conditions of these reactors. The two-phase simulation was shown to provide a good description of Sasol M5 wax, although it is unclear if this arises due to additional processing steps involved in producing this wax fraction. In all cases, the model rate constants were within the same order of magnitude. This highlights that the kinetics alone do not determine the product distribution and that chemical and vapour-liquid equilibrium are substantial contributing factors. 


\section{Chapter 8}

\section{Conclusions and Recommendations}

\subsection{Conclusions}

The objective of this thesis was to develop a kinetic model for FT synthesis that combines the interaction between reaction equilibrium, kinetics and vapour-liquid equilibrium to describe the observed reaction behaviour. Based on the work in Chapter 3-7, the following conclusions can be drawn regarding the development of the kinetic model.

In Chapter 3, a physical property and thermodynamic database for FT synthesis was developed. The lack of physical property data for high carbon number n-paraffins and 1-olefins meant that these data needed to be accurately estimated for chemical and phase equilibrium calculations in FT synthesis. For these calculations, the critical and ideal gas properties specifically are required. The Constantinou and Gani group contribution method provided an accurate estimation of these properties upon regression of experimental data for $C_{1}-C_{29}$ n-paraffins and $C_{2}-C_{9}$ and $C_{11}$ 1-olefins. The group contributions were chosen as the adjustable parameters with a good fit to data obtained for these carbon number n-paraffins and 1-olefins. Reliable estimates of the critical and ideal gas properties for higher carbon number n-paraffins and 1-olefins can therefore be expected.

To provide a prediction of the phase behaviour of FT synthesis, the PR-EOS has most commonly been used and as such, was the most appropriate choice. The accuracy of the prediction of the phase behaviour of FT synthesis by the PR-EOS is, however, dependent on its description of the pure component vapour pressure since this determines the behaviour of the reaction mixture. The latter is determined by the choice of alpha function, which needs to be modified to allow the PR-EOS to be extended to describe the behaviour of heavy hydrocarbons. Modification of the PR-EOS, by using the alpha function of Twu et al. (1995), enabled its behaviour to be extended 
to the description of heavy hydrocarbon. As such, the PR-EOS should be expected to provide an accurate approximation of the phase behaviour of the $\mathrm{FT}$ reaction mixture.

The work in Chapter 3, laid the foundation for the development of the kinetic model. The work in Chapter 4 focused on developing a model which relies on the equilibrium aspects of the reactions involved in FT synthesis. The equilibrium control in the product distribution is attributed to the rapid rate of chain growth in comparison to the rate of $\mathrm{CO}$ hydrogenation, consistent with findings in literature (van Helden et al., 2017; Allie \& Nyathi, 2019; Hensen et al., 2020). The latter is the rate-determining reaction step in the reaction pathway. This allowed the rate expression for each reaction step to be formulated as an equilibrium-limited process for the formation of n-paraffins and 1-olefins, the main products of FT synthesis. By following this strategy in the kinetic model development, the observed reaction behaviour of FT synthesis could successfully be accounted for. The polymerisation character of the reaction was preserved and consequently, no assumptions regarding the carbon number dependence of the reaction rate was required. The model only consists of six adjustable parameters i.e. rate constants $\mathrm{k}_{\mathrm{j}}$.

The model could effectively capture the changes in product selectivity and the product distribution as a function of process conditions ( $\mathrm{CO}$ conversion, temperature, pressure and $\mathrm{H}_{2} / \mathrm{CO}$ feed ratio) and agreed with findings reported in literature (Iglesia et al., 1993: Espinoza et al., 1999;: Tucker \& van Steen, 2019). These changes could all be explained by considering the equilibrium aspects of the reactions involved. Increases in temperature favour dehydrogenation rather than hydrogenation consequently shifting the selectivity of FT synthesis to 1-olefins rather than n-paraffins. Increases in pressure shifts the selectivity of FT synthesis towards products of higher carbon number since chain growth results in a reduction in the number of moles (Le Chatelier's principle). The model could predict changes in product selectivity and the product distribution as a function of $\mathrm{CO}$ conversion that agreed with the trends in the work of Tucker \& van Steen (2019). This will prove helpful in determining an operating CO conversion which maximises wax selectivity.

The relationship between the model rate constants and its influence on model output behaviour was investigated in Chapter 5. To provide insight into this relationship, a one-at-a-time and derivativebased sensitivity analysis was performed on the selectivity to $C_{1}, C_{2}, C_{3}, C_{4}, C_{5+}$ and $C_{2}$ as a function of $\mathrm{CO}$ conversion. The one-at-a-time analysis confirmed that $\mathrm{CO}$ hydrogenation is rate-determining for both the FT and WGS reactions. To provide insight regarding which rate constants could be regressed from product selectivity as a function of $\mathrm{CO}$ conversion data, the relative sensitivity of the product selectivity with respect to each rate constants was calculated in the derivative-based analysis. A high degree of correlation exists between the rate constants. This means that if product selectivity as a function of $\mathrm{CO}$ conversion data is used to determine the rate constants then the current rate constant set needs to be replaced with a reduced rate constant 
set. The development of a reduced rate constant set would involve developing a set of correlations using a moment analysis.

An alternative strategy was investigated where total hydrocarbon product distribution data at constant CO conversion is used instead to determine the model rate constants. A sensitivity analysis on the product distribution with respect to each rate constant revealed that meaningful values of $k_{1}-k_{5}$ could be determined, provided that the CO conversion is below $60 \%$. Over the same CO conversion range the model is independent of $k_{6}$, the WGS rate constant. As such the number of rate constants that need to be estimated would have to be reduced by approximating the value of $k_{6}$ using data in literature.

In Chapter 6, the predictive ability of the kinetic model was assessed. Emphasis was placed on the ability of the model to predict changes in the product distribution with temperature in both a FBR and slurry reactor. The model was shown to effectively describe the reaction behaviour in both reactor types which agrees with the findings of Visconti et al. (2016). In the sensitivity analysis, it was established that the product distribution is only sensitive to $k_{1}-k_{5}$. As such, the value of $k_{6}$ was approximated using a relationship from the work of Rafiq et al. (2011). A good model fit, quantified by the small error in the estimates of $k_{1}-k_{5}$, was obtained particularly for a CO conversion up to approximately $30 \%$. Higher variability in the estimates of $k_{1}-k_{5}$ was obtained at higher $\mathrm{CO}$ conversions despite the good qualitative fit of the model to the data of Visconti et al. (2016). This emphasises the importance of estimating rate constants at conditions where the product distribution is most sensitive. The temperature-dependence of the rate constants could be described by the Arrhenius expression provided that the dataset had been measured at a consistent set of operating conditions. The good model fit to data further highlights the strength of modelling the kinetics of FT synthesis by considering the equilibrium aspects of the reactions involved.

Most of the work in this thesis concentrated on modelling the FT reactions in the vapour phase only. In Chapter 7 , the effect of liquid formation on product selectivity and the product distribution was evaluated. The effect of liquid formation on the kinetic behaviour of FT synthesis was modelled by assuming that the vapour and liquid phases are in equilibrium. The choice of species activity as the kinetic driving force means that the kinetic model could be applied in both the vapour and liquid phases, with the magnitude of the rate constants affected by phase changes.

For a system in which the vapour phase dominates, a small amount of liquid was shown to alter selectivity. Single-phase simulations in which the reactions occur in the vapour phase only predicts a higher selectivity towards products of carbon number in the diesel product grade $\left(C_{10}-C_{20}\right)$. More correctly, two-phase simulations in which the reactions occur in both the vapour and liquid phases predicts that FT synthesis is selective towards products of carbon number in the wax product 
grade $\left(\mathrm{C}_{21+}\right)$. For $\mathrm{CO}$ conversions below $20 \%$, single-phase simulations are valid. Between a CO conversion of $20-90 \%$, it is essential to account for liquid formation. Above a CO conversion of $90 \%$, both the single- and two-phase simulation favour the formation of methane, the most thermodynamically favoured product.

The predictions of the single- and two-phase simulations were assessed by comparison to the wax product from the iron-catalysed FT processes of Sasol. The single-phase simulation gave a good prediction of the wax product from ARGE tubular and slurry reactors. This finding is attributed to the operating conditions of these reactors. The two-phase simulation was shown to give a good description of the Sasol M5 wax fraction. This demonstrates that both simulations are useful to describe the wax product distribution from LTFT reactors.

The kinetic model developed in this thesis can therefore describe FT synthesis in a lightweight and tractable manner. It can be concluded that the objective of this thesis has been met. The ability of the model to predict changes in product selectivity and the product distribution as a function of process conditions make it an important tool for engineers involved in the design and simulation of LTFT reactors to ensure maximum wax selectivity is obtained for fuel production.

\subsection{Recommendations}

The work in this thesis makes it possible for further investigations to be performed in areas of reaction kinetics, reactor design and process flowsheet development.

\subsubsection{Reaction Kinetics}

In this thesis, it was demonstrated that the reactions involved in the $\mathrm{CO}$ hydrogenation system could be modelled as equilibrium-limited processes. This technique could also be extended to model the kinetics of other gas-to-liquid processes like the production of methanol from syngas, which also possibly involves the hydrogenation of $\mathrm{CO}$ to a $\mathrm{CH}_{2}$ intermediate on a metal catalyst.

The work of Visconti et al. (2009) compared the product distributions from $\mathrm{CO}$ and $\mathrm{CO}_{2}$ hydrogenation and indicated that the same intermediates are involved in the formation of hydrocarbons. Allie \& Nyathi (2019) confirmed that $\mathrm{CO}_{2}$ hydrogenation proceeds via the reverse water gas shift reaction, followed by $\mathrm{CO}$ hydrogenation. The work in this thesis could thus aid in developing a kinetic model for $\mathrm{CO}_{2}$ hydrogenation. Such a model may help to improve the understanding of $\mathrm{CO}_{2}$ hydrogenation. This will also aid in its prospect as a possible process route to produce liquid fuels and chemicals to assist in mitigating the continued rise of anthropogenic $\mathrm{CO}_{2}$ emissions.

While the kinetic model developed in this work was tested under LTFT conditions, it may also be 
an important tool to describe the reaction behaviour of high temperature FT (HTFT) synthesis. The formation of branched and aromatic compounds could be described by including n-paraffin and 1-olefin isomerisation. This may aid in providing insight regarding the conditions which maximise the production of gasoline and naptha.

\subsubsection{Reactor Design}

With a reliable FT kinetic model, the simulation and design of LTFT and possibly, HTFT reactors can be improved. The major challenge to FBRs used for LTFT synthesis is temperature control. The interaction of kinetics and VLE and the ability to provide a prediction of liquid formation will aid in an improved prediction of the heat transfer rate. This is since the heat transfer characteristics of the fixed bed reactor are dependent on the presence of liquid. The interaction between reaction, heat transfer, VLE, condensation and evaporation could lead to improvements in simulating the behaviour of FBRs (Glasser et al., 2012). Similarly, the prediction of liquid formation can improve the description of gas-liquid hydrodynamics and mass transfer effects in slurry reactors. Ultimately, this may contribute to the improved understanding and the accelerated development of conventional and innovative reactor types.

\subsubsection{Process Flowsheet Development}

An improved description of FT kinetics means that emphasis can be placed on simulating and optimising processes converting coal, gas and particularly renewable feeds like biomass to liquid fuels. In the case of the latter, small-scale operation is attractive for biomass-to-liquid plants, but significant challenges exist to ensure a plant of this size is economically viable due to the costly syngas production step. The work of Tucker \& van Steen (2019) indicates that these challenges can be overcome by operating the LTFT reactor at the highest CO conversion possible which maximises liquid fuel production. The kinetic model developed in this thesis could therefore be used to determine the optimal operating $\mathrm{CO}$ conversion where syngas is utilised as efficiently as possible and consequently maximises wax selectivity (Geerlings et al., 1999). This could be paired with the models of Le Grange (2009) to describe the production of high quality diesel. This may help to make the process more profitable, attractive and aid in the shift from fossil fuels to more environmentally friendly and sustainable alternatives. 


\section{Bibliography}

Accolla, Carlo. 2006. The Development of Fischer-Tropsch Wax Hydrocracking Reactor Model. M.Phil. thesis, University of Cape Town.

Ahón, Victor R., Costa Jr., Esly F., Monteagudo, Jorge E. P., Fontes, Carlos E., Biscaia Jr., Evaristo C., \& Lage, Paulo L. C. 2005. A comprehensive mathematical model for the FischerTropsch synthesis in well-mixed slurry reactors. Chemical Engineering Science.

Allie, Farhana, \& Nyathi, Jabulani. 2019. Micro-kinetic comparison of $\mathrm{CO}$ and $\mathrm{CO}_{2}$ hydrogenation over Fe (100). Tech. rept. University of Cape Town.

Bukur, Dragomir B., Pan, Zhendong, Ma, Wenping, Jacobs, Gary, \& Davis, Burtron H. 2012. Effect of $\mathrm{CO}$ Conversion on the Product Distribution of a $\mathrm{Co} / \mathrm{Al}_{2} \mathrm{O}_{3}$ Fischer-Tropsch Catalyst Using a Fixed Bed Reactor. Catalysis letters.

Caldwell, L., \& van Vuuren, D. S. 1986. On the formation and composition of the liquid phase in Fischer-Tropsch reactors. Chemical engineering science.

Claeys, Micheael, \& van Steen, Eric. 2004. Basic studies. Pages 601-680 of: Studies in surface science and catalysis, vol. 152. Elsevier.

Davis, Burton H. 1992. The two-alpha value for iron Fischer-Tropsch catalysts: Fact or Fiction? ACS, Division of Fuel Chemistry, Preprints.

Derevich, I. V., Ermolaev, V. S., \& Mordkovich, V. Z. 2008. Liquid-Vapor Thermodynamic Equilibrium in Fischer-Tropsch Synthesis Products. Theoretical Foundations of Chemical Engineering.

Dictor, R. A., \& Bell, A. T. 1986. A comparison of the activity, selectivity and kinetics of several iron-based Fischer-Tropsch catalysts. Am. Chem. Soc., Div. Pet. Chem., Prep.; (United States).

Edgar, Thomas F., Himmelblau, David M., \& Lasdon, Leon S. 2001. Optimization of Chemical Processes. McGraw-Hill. 
Elliot, Richard J., \& Lira, Carl T. 2012. Introductory Chemical Engineering Thermodynamics. Pearson Education Inc.

Ermolaev, Vladim S., Gryaznov, Kirill O., Mitberg, Eduard B., Mordkovich, Vladmir Z., \& Tretyakov, Valentin F. 2015. Laboratory and pilot plant fixed-bed reactors for Fischer-Tropsch synthesis: Mathematical modeling and experimental investigation. Chemical Engineering Science.

Espinoza, R. L., Steynberg, A. P., Jager, B., \& Vosloo, A. C. 1999. Low temperature FischerTropsch synthesis from a Sasol perspective. Applied Catalysis A: General.

Fernandes, F. A. N. 2005. Polymerization Kinetics of Fischer-Tropsch Reaction on Iron Based Catalysts and Product Grade Optimization. Chemical Engineering \& Technology.

Fernandes, Fabiano A. N. 2006. Modeling and Product Grade Optimization of Fischer-Tropsch Synthesis in a Slurry Reactor. Industrial \& Engineering Chemistry Research.

Fogler, Harold Scott. 1999. Elements of Chemical Reaction Engineering. Pearson Education, Inc.

Förtsch, Dieter, Pabst, Kyra, \& Gross-Hardt, Edwin. 2015. The product distribution in FischerTropsch synthesis: An extension of the ASF model to describe common deviations. Chemical Engineering Science.

Fry, Matthew Ryan. 2010. Development of a Generalised Kinetic Model for the Combustion of Hydrocarbon Fuels. M.Phil. thesis, University of Cape Town.

Geerlings, J. J. C., Wilson, J. H., Kramer, G. J., Kuipers, H. P. C. E., Hoek, A., \& Huisman, H. M. 1999. Fischer-Tropsh Technology - from active site to commercial process. Applied Catalysis: A General.

Glasser, David, Hildebrandt, Diane, Liu, Xinying, Lu, Xiaojun, \& Masuku, Cornelius. 2012. Recent advances in understanding the Fischer-Tropsh synthesis (FTS) reaction. Current Opinion in Chemical Engineering.

Heath, Michael T. 2002. Scientific Computing: An Introductory Survey. McGraw-Hill.

Hensen, Emiel J. M., Zjilstra, Bart, Broos, Robin J. P., Chen, Wei, \& Filot, Ivo A. W. 2020. First-principles based microkinetic modeling of transient kinetics of $\mathrm{CO}$ hydrogenation on cobalt catalysts. Catalysis Today.

Hildebrandt, Diane, Xiaowei, Zhu, Xiaojun, Lu, Liu, Xinying, \& Glasser, David. 2010. Study of 
Radial Heat Transfer in a Tubular Fischer-Tropsch Synthesis Reactor. Industrial \& Chemistry Engineering Research.

Hillestad, Magne, Ostadi, Mohammad, \& Rytter, Erling. 2016. Evaluation of kinetic models for Fischer-Tropsch cobalt catalysts in a plug flow reactor. Chemical Engineering Research and Design.

Horiuti, lurô, \& Polanyi, Michael. 1934. Exchange reactions of hydrogen on metallic catalysts. Transactions of the Faraday Society.

Iglesia, Enrique. 1993. Selectivity Control and Catalyst Design in the Fischer-Tropsch Synthesis: Sites, Pellets and Reactors. Advances in catalysis.

Iglesia, Enrique, Reyes, Sebastian C., \& Madon, Rostam J. 1991. Transport-enhanced $\alpha$-olefin readsorption pathways in Ru catalyzed hydrocarbon synthesis. Journal of Catalysis.

Iglesia, Enrique, Reyes, Sebastian C., \& Soled, Stuart L. 1993. Reaction-transport selectivity models and the design of Fischer-Tropsch catalysts. Chemical Industries-New York-Marcel Dekker.

Jager, B. 1997. Developments in Fischer-Tropsch technology. In: Studies in surface science and catalysis. Elsevier.

Jess, Andreas, \& Kern, Christoph. 2009. Modeling of Multi-Tubular Reactors for Fischer-Tropsch Synthesis. Chemical and Engineering \& Technology: Industrial Chemistry-Plant EquipmentProcess Engineering Biotechnology.

Kamal K. Pant, Sonal, \& Upadhyayula, Sreedevi. 2019. Detailed kinetics of Fischer-Tropsch synthesis over Fe-Co bimetallic catalyst considering chain length dependent olefin desorption. Fuel.

Khazali, Ashcaan Tendo. 2018. A Study of Configurational Alternatives of a Gas-To-Liquids Process based on Fischer-Tropsch Technology. M.Phil. thesis, University of Cape Town.

Kittrell, J. R. 1970. Mathematical modeling of chemical reactions. In: Advances in Chemical Engineering. Elsevier.

Kobori, Yoshihiro, Yamasaki, Hirofumi, Naito, Shuichi, Onishi, Takaharu, \& Tamaru, Kenzi. 1982. Mechanistic study of carbon monoxide hydrogenation over ruthenium catalysts. Journal of the Chemical Society, Faraday Transactions 1: Physical Chemistry in Condensed Phases.

Kroschwitz, Jacqueline I., \& Mark, Herman Francis. 1996. Encyclopedia of polymer science and engineering. John Wiley \& Sons. 
Kuipers, E. W., Vinkenburg, I. H., \& Oosterbeek, H. 1995. Chain lenght dependence of $\alpha$-olefin readsorption in Fischer-Tropsch synthesis. Journal of Catalysis.

Kwack, Seung-Ho, Park, Myung-June, Bae, Jong Wook, Ha, Kyoung-Su, \& Jun, Ki-Won. 2011. Development of a kinetic model of the Fischer-Tropsch synthesis reaction with a cobalt-based catalyst. Reaction Kinetics, Mechanisms and Catalysis.

Laxmi Narasimhan, C. S., Thybaut, Joris W., Martens, Johan A., Jacobs, Pierre A., Denayer, Joeri F., \& Marin, Guy B. 2006. A Unified Single-Event Microkinetic Model for Alkane Hdryoconversion in Different Aggregration States on Pt/H-USY-Zeolites. The Journal of Physical Chemistry $B$.

Le Grange, Philip. 2009. Models for the hydrocracking of Fischer-Tropsch derived waxes. M.Phil. thesis, University of Cape Town.

Leckel, Dieter. 2005. Hydrocracking of Iron-Catalyzed Fischer-Tropsch Waxes. Energy \& Fuels.

Leckel, Dieter, \& Liwanga-Ehumbu, Maurice. 2006. Diesel-Selective Hydrocracking of an IronBased Fischer-Tropsch Wax Fraction $\left(\mathrm{C}_{15}-\mathrm{C}_{45}\right)$ Using a $\mathrm{MoO}_{3}$-Modified Noble Metal Catalyst. Energy \& Fuels.

Lozano-Blanco, G., Thybaut, J. W., Surla, K., Galtier, P., \& Marin, G. B. 2006. Fischer-Tropsch Synthesis: Development of a Microkinetic Model for Metal Catalysis. Oil \& Gas Science and Technology-Revue de I'IFP.

Marano, John J., \& Holder, Gerald D. 1997a. Characterization of Fischer-Tropsch liquids for vapor-liquid equilibria calculations. Fluid Phase Equilibria.

Marano, John J., \& Holder, Gerald D. 1997b. General Equation for Correlating the Thermophysical Properties of n-Paraffins, n-Olefins and Other Homologous Series. 2. Asymptotic Behavior Correlations for PVT Properties. Industrial \& Engineering Chemistry Research.

Marano, John J., \& Holder, Gerald D. 1997c. General Equation for Correlating the Thermophysical Properties of n-Paraffins, n-Olefins and Other Homologous Series. 2. Asymptotic Behavior Correlations for Thermal and Transport Properties. Industrial \& Engineering Chemistry Research.

Marano, John J., \& Holder, Gerald D. 1997d. General Equation for Correlating the Thermophysical Propeties of n-Paraffins, n-Olefins and Other Homologous Series. 1. Formalism for Developing Asymptotic Behaviour Correlations. Industrial \& Engineering Chemistry Research.

Marchese, Marco, Heikkinen, Niko, Giglio, Emanuele, Lanzini, Andrea, Lehtonen, Juha, \& 
Reinikainen, Matti. 2019. Kinetic Study Based on the Carbide Mechanism of a Co-Pt $/ \gamma-\mathrm{Al}_{2} \mathrm{O}_{3}$ Fischer-Tropsch Catalyst Tested in a Laboratory-Scale Tubular Reactor. Catalysts.

Masuku, Cornelius Mduduzi, Hildebrandt, Diane, \& Glasser, David. 2011. The role of vapour-liquid equilibrium in Fischer-Tropsch product distribution. Chemical Engineering Science.

Masuku, Cornelius Mduduzi, Ma, Wenping, Hildebrandt, Diane, Glasser, David, \& Davis, Burtron H. 2012a. A vapor-liquid equilibrium thermodynamic model for a Fischer-Tropsch reactor. Fluid Phase Equilibria.

Masuku, Cornelius Mduduzi, Hildebrandt, Diane, \& Glasser, David. 2012b. Olefin pseudoequilibrium in the Fischer-Tropsch reaction. Chemical Engineering Journal.

Méndez, César, Ancheyta, Jorge, \& Trejo, Fernando. 2017. Modeling of Catalytic Fixed-Bed Reactors for Fuels Production by Fischer-Tropsch Synthesis. Energy \& fuels.

Mims, C. A., Krajewski, J. J., Rose, K. D., \& Melchior, M. T. 1990. Residence times and coverage by surface intermediates during the Fischer-Tropsch synthesis. Catalysis Letters.

Möller, Klaus, le Grange, Philip, \& Accolla, Carlo. 2009. A Two-Phase Reactor Model for the Hydrocracking of Fischer-Tropsch-Derived Wax. Industrial \& Engineering Chemistry Research.

Montgomery, Douglas C., \& Runger, George C. 2014. Applied Statistics and Probability for Engineers. John Wiley \& Sons.

Mousavi, Shabbir, Zamaniyan, Akbar, Irani, Mohammad, \& Rashidzadeh, Mehdi. 2015. Generalized kinetic model for iron and cobalt based Fischer-Tropsch catalysts: Review and model evaluation. Applied Catalysis A: General.

Mthombeni, Bongani. 2009. Modeling Fischer-Tropsch synthesis in two-phase, continuous, wellmixed slurry reactors. M.Phil. thesis, University of Cape Town.

Norval, G. W., \& Phillips, M. J. 1990. Application of Equilibrium Analysis to a Fischer-Tropsch Product. Journal of Catalysis.

Norval, Graeme W. 2008. Notes on the issues of equilibrium in the Fischer-Tropsch synthesis. The Canadian Journal of Chemical Engineering.

Norval, Graeme W., Phillips, M. Jane, Missen, Ronald W., \& Smith, William R. 1992. Identification and application of partial chemical equilibria in reactor modeling. AIChE journal.

Patzlaff, J., Liu, Y., Graffmann, C., \& Gaube, J. 1999. Studies on product distributions of iron and cobalt catalyzed Fischer-Tropsch synthesis. Applied Catalysis A: General. 
Philippe, Régis, Lacroix, Maxime, Dreibine, Lamia, Pham-Huu, Cuong, Eduoard, David, Savin, Sabine, Luck, Francis, \& Schweich, Daniel. 2009. Effect of structure and thermal properties of a Fischer-Tropsch catalyst in a fixed-bed. Catalysis Today.

Pichler, H., \& Schulz, H. 1970. Recent knowledge in the field of the synthesis of hydrocarbons from $\mathrm{CO}$ and $\mathrm{H}_{2}$. chemical engineering technology.

Poling, Bruce E., Prausnitz, John M., \& O'Connell, John P. 2001. The Properties of GASES AND LIQUIDS. McGraw-Hill Companies, Inc.

Press, William H., Teukolsky, Saul A., Vetterling, William T., \& Flannery, Brian P. 1992. Numerical Recipes in Fortran 77: The Art of Scientific Computing. Cambridge University Press.

Privat, Romain, \& Jaubert, Jean-Noël. 2012. Thermodynamic models for the prediction of petroleum-fluid phase behaviour. Crude-Oil Emulsions-Composition Stability and Characterization.

Puskas, I., \& Hurlbut, R. S. 2003. Comments about the causes of deviations from the AndersionSchulz-Flory distribution of the Fischer-Tropsch reaction products. Catalysis Today.

Qian, Weixin, Zhang, Haitao, Ying, Weiyong, \& Fang, Dingye. 2013. The comprehensive kinetics of Fischer-Tropsch synthesis over a Co/AC catalysts on the basis of the CO insertion mechanism. Chemical engineering journal.

Rafiq, Muhammad Hamid, Jakobsen, Hugo Atle, Schmid, Rudolf, \& Hustad, Johan Einar. 2011. Experimental studies and modeling of a fixed bed reactor for Fischer-Tropsch synthesis using biosyngas. Fuel processing technology.

Saeidi, Samrand, Nikoo, Maryam Khoshtinat, Mirvakili, Azadeh, Bahrani, Samaneh, Amin, Nor Aishah Saidina, \& Rahimpour, Mohammad Reza. 2015. Recent advances in reactors for lowtemperature Fischer-Tropsch synthesis: process intensification perspective. Reviews in Chemical Engineering.

Sage, Valérie, Bhatelia, Tejas, Li, Chao'en, Sun, Yong, Hazewinkel, Philip, \& Burke, Nicholas. 2014. Chain length dependent olefin re-adsorption model for Fischer-Tropsch synthesis over $\mathrm{Co}-\mathrm{Al}_{2} \mathrm{O}_{3}$. Fuel Processing Technology.

Satterfield, Charles N., Huff Jr, George A., Stenger, Harvey G., Carter, James L., \& Madon, Rostam J. 1985. A comparison of Fischer-Tropsch synthesis in a fixed-bed reactor and in a slurry reactor. Industrial \& engineering chemistry fundamentals. 
Schulz, Hans, \& Claeys, Michael. 1999. Kinetic modelling of Fischer-Tropsch product distributions. Applied Catalysis A: General.

Schulz, Hans, van Steen, Eric, \& Claeys, Michael. 1994. Selectivity and mechanism of FischerTropsch synthesis with iron and cobalt catalysts. Studies in Surface Science and Catalysis.

Schwan, Peter. 2001. Transient Analysis of Diffusion and Adsorption under Reaction Conditions. Ph.D. thesis, University of Cape Town.

Sealy, Sarah Jane. 1996. THE DEVELOPMENT OF A MODEL FOR HIGH PRESSURE PROPENE OLIGOMERISATION OVER H-ZSM-5. Ph.D. thesis, University of Cape Town.

Sharrock, Cayle J., \& Coetzer, Roelof. 2007. Selecting Robust Kinetic Models on Noisy Data Using the Boostrap. International Journal of Chemical Engineering.

Sie, S. T. 1998. Process development and scale up: IV. Case history of the development of a Fischer-Tropsch synthesis process. Reviews in Chemical Engineering.

Sie, S. T., \& Krishna, R. 1999. Fundamentals and selection of advanced Fischer-Tropsch reactos. Applied Catalysis A: General.

Sie, S. T., Senden, M. M. G., \& van Wechem, H. M. H. 1991. Conversion of natural gas to transportation fuels via the Shell middle distillate synthesis process (SMDS). Catalysis Today.

Smith, William R., \& Missen, Ronald W. 1988. Strategies for solving the chemical equilibrium problem and an efficient microcomputer-based algorithm. The Canadian Journal of Chemical Engineering.

Subiranas, Alba Mena. 2009. Combining Fischer-Tropsch synthesis (FTS) and hydrocarbon reactions in one reactor. KIT Scientific Publishing.

Teng, Bo-Tao, Chang, Jie, Zhang, Cheng-Huaa, Cao, Dong-Bo, Yang, Jun, Liu, Ying, Guo, XiaoHui, Xiang, Hong-Wei, \& Li, Yong-Wang. 2006. A comprehensive kinetics model of FischerTropsch synthesis over an industrial Fe-Mn catalyst. Applied Catalysis A: General.

Todic, Branislav, Ma, Wenping, Jacobs, Gary, Davis, Burtron H., \& Bukur, Dragomir B. 2014. CO-insertion mechanism based kinetic model of the Fischer-Tropsch synthesis reaction over Re-promoted Co catalyst. Catalysis Today.

Tucker, Chelsea L., \& van Steen, Eric. 2019. Activity and selectivity of a cobalt-based FischerTropsch catalyst operating at high conversion for once-through biomass-to-liquid operation. Catalysis Today. 
Turton, Richard, Bailie, Richard C., Whiting, Wallace B., \& Shaeiwitz, Joseph A. 2009. Analysis, Synthesis, and Design of Chemical Processes. Prentic Hall.

Twu, Chorn H., Coon, John E., \& Cunningham, John R. 1994. A generalized vapor pressure equation for heavy hydrocarbons. Fluid Phase Equilibria.

Twu, Chorng H., Bluck, David, Cunningham, John R., \& Coon, John E. 1991. A cubic equation of state with a new alpha function and a new mixing rule. Fluid Phase Equilibria.

Twu, Chorng H., Coon, John E., \& Cunningham, John R. 1995. A new generalized alpha function for a cubic equation of state Part 1. Peng-Robinson equation. Fluid Phase Equilibria.

van der Laan, Gerard P. 1999. Kinetics, selectivity and scale up of the Fischer-Tropsch synthesis. Groningen.

van Helden, Pieter, van den Berg, Jan-Albert, Petersen, Melissa A., van Rensburg, Werner Janse, Ciobica, Ionel M., \& van de Loosdrecht, Jan. 2017. Computational investigation of the kinetics and mechanism of the initial steps of the Fischer-Tropsch synthesis on cobalt. Faraday Discussions.

van Steen, E., Claeys, M., Möller, K. P., \& Nabaho, D. 2018. Comparing a cobalt-based catalyst with iron-based catalysts for the Fischer-Tropsch XTL-process operating at high conversion. Applied Catalysis A: General.

Varma, Arvind, Morbidelli, Massimo, \& Wu, Hua. 2005. Parametric sensitivity in chemical systems. Cambridge University Press.

Visconti, Carlo Giorgio. 2014. Vapor-liquid equilibria in the low-temperature Fischer-Tropsch synthesis. Industrial \& Engineering Chemistry Research.

Visconti, Carlo Giorgio, Tronconi, Enrico, Lietti, Luca, Zennaro, Roberto, \& Forzatti, Pio. 2007. Development of a complete kinetic model for the Fischer-Tropsch synthesis over $\mathrm{Co} / \mathrm{Al}_{2} \mathrm{O}_{3}$ catalysts. Chemical Engineering Science.

Visconti, Carlo Giorgio, Lietti, Luca, Tronconi, Enrico, Forzatti, Pio, Zennaro, Roberto, \& Finocchio, Elisabetta. 2009. Fischer-Tropsch synthesis on a $\mathrm{Co} / \mathrm{Al}_{2} \mathrm{O}_{3}$ catalyst with $\mathrm{CO}_{2}$ containing syngas. Applied Catalysis A: General.

Visconti, Carlo Giorgio, Tronconi, Enrico, Lietti, Luca, Forzatti, Pio, Rossini, Stefano, \& Zennaro, Roberto. 2011. Detailed kinetics of the Fischer-Tropsch synthesis on cobalts catalysts based on $\mathrm{H}$-assisted $\mathrm{CO}$ activation. Topics in Catalysis. 
Visconti, Carlo Giorgio, Lietti, Luca, Tronconi, Enrico, \& Rossini, Stefano. 2016. Kinetics of Low Temperature Fischer-Tropsch Synthesis on Cobalt Catalysts: Are both slurry autoclave and tubular packed-bed reactors adequate to collect relevant data at lab-scale? The Canadian Journal of Chemical Engineering.

Wang, Yi-Ning, Xu, Yuan-Yuan, Li, Yong-Wang, Zhao, Yu-Long, \& Zhang, Bi-Jiang. 2003a. Heterogeneous modeling for fixed-bed Fischer-Tropsch synthesis: Reactor model and its applications. Chemical Engineering Science.

Wang, Yi-Ning, Ma, Wen-Ping, Lu, Yi-Jun, Yang, Jun, Xu, Yuan-Yuan, Xiang, Hong-Wei, Li, Yong-Wang, Zhao, Yu-Long, \& Zhang, Bi-Jiang. 2003b. Kinetics modelling of Fischer-Tropsch synthesis over an industrial Fe-Cu-K catalyst. Fuel.

Yamasaki, Hirofumi, Kobori, Yoshihiro, Naito, Shuichi, Onishi, Takaharu, \& Tamaru, Kenzi. 1981. Infrared study of the reaction of $\mathrm{H}_{2}+\mathrm{CO}$ on a $\mathrm{Ru} / \mathrm{SiO}_{2}$ catalyst. Journal of the Chemical Society, Faraday Transactions 1: Physical Chemistry in Condensed Phases. 


\section{Appendix A}

\section{Validation of Model Development Assumptions}

\section{A.1 Validation of the Pseudo-Steady State Assumption}

An important assumption made during the development of the kinetic model is that the pseudospecies $\mathrm{CH}_{2}$ is at pseudo-steady state. This arises if it has a large specific reaction rate (Fogler, 1999). Consequently, the net reaction rate for $\mathrm{CH}_{2}$ is zero. An explicit expression, Equation 4.8, is then derived for the activity of $\mathrm{CH}_{2}$. The result of this procedure is that the activity of $\mathrm{CH}_{2}$ is known in advance, depending on the activity of the other species present. As such be it can be eliminated from the rate expressions in Table 4.2. Two checks, however, need to be made:

1. What is the activity of $\mathrm{CH}_{2}$ as a function of $\mathrm{CO}$ conversion?

2. Is the net rate for $\mathrm{CH}_{2}$ indeed zero?

Check 1 is necessary to ensure that the activity of $\mathrm{CH}_{2}$ is bound by the carbon, hydrogen and oxygen balances and that the pseudo-steady state assumption gives rise to behaviour that is physical consistent i.e. mass is conserved. Check 2 is performed as a double-check that the net rate for $\mathrm{CH}_{2}$ is indeed zero and that the pseudo-state assumption is not violated at all $\mathrm{CO}$ conversions. This analysis is carried out at a temperature of $220^{\circ} \mathrm{C}$, pressure of $20 \operatorname{bar}(\mathrm{a})$ and $\mathrm{H}_{2} / \mathrm{CO}$ feed ratio of 2.1 .

In Figure A.1a, the activity of $\mathrm{CH}_{2}$ is compared relative to that of $\mathrm{CO}$ and $\mathrm{H}_{2}$ as a function of $\mathrm{CO}$ conversion. This is since it is coupled to $\mathrm{CO}$ and $\mathrm{H}_{2}$ by reaction step 1 , which then controls the process of chain growth. The activity of $\mathrm{CH}_{2}$ responds as given by the reaction kinetics. There is 
initially a rise in the activity of $\mathrm{CH}_{2}$. This occurs due to its formation in reaction step 1 , reaching a local maximum between a $\mathrm{CO}$ conversion of $2-3 \%$. Thereafter, the activity of $\mathrm{CH}_{2}$ decreases rapidly until a CO conversion of $10 \%$. This arises because of its consumption in the $n$-paraffin and 1-olefin chain growth reactions. At a $\mathrm{CO}$ conversion of $10 \%$, the activity of $\mathrm{CH}_{2}$ curve changes concavity and decreases linearly. This indicates that the reverse $n$-paraffin and 1-olefin chain growth reactions are favoured. In other words, $\mathrm{CH}_{2}$ is formed in reaction steps 3-5. The steady decrease is then attributed to the consumption of $\mathrm{CH}_{2}$ towards methane. The pseudo-species $\mathrm{CH}_{2}$ acts as an effective correlator for modelling the chemical feedback in the FT reaction system.

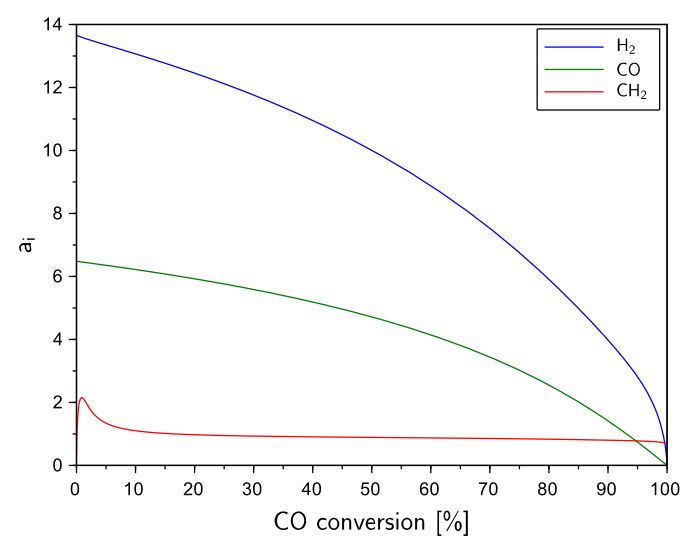

(a) Activity of $\mathrm{CH}_{2}$ as a function of $\mathrm{CO}$ conversion

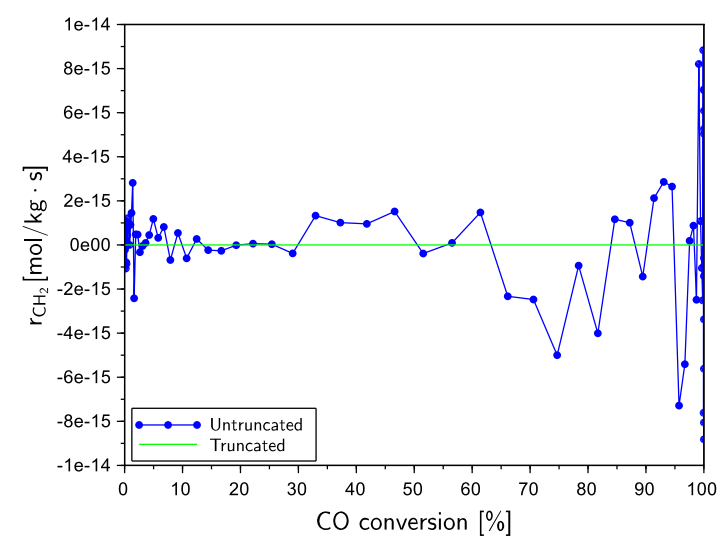

(b) Net rate of $\mathrm{CH}_{2}$ as a function of $\mathrm{CO}$ conversion

Figure A.1: Validation of the pseudo-steady state approximation applied to $\mathrm{CH}_{2}$

It is also clear from Figure A.1a that relative to $\mathrm{CO}$ and $\mathrm{H}_{2}$, the activity of $\mathrm{CH}_{2}$ given by Equation 4.8 is reasonable and consistent with that of a reaction product. Further, the pseudo-steady state assumption is shown to be physical consistent by the closure of the element balances in Table 4.5 . In Figure A.1b, the net rate for $\mathrm{CH}_{2}$ is shown to be equal to zero, within the limit of machine precision. The net rate for $\mathrm{CH}_{2}$ as a function of $\mathrm{CO}$ conversion, however, shows numerically erratic behaviour in Figure A.1b. This arises because of error coupling during the integration of the differential equations. However, by truncating the net reaction rate for $\mathrm{CH}_{2}$ to 8 decimal point precision, it is clear that the pseudo-steady state approximation is not violated across all CO conversions in Figure A.1b, It is noted that only one set of reaction conditions are used in this analysis but the conclusions drawn should be generalisable across all LTFT synthesis conditions. Both Check 1 and 2 are thus satisfied and the pseudo-steady state approximation can safely be applied. 


\section{A.2 Validation of the Choice of Maximum Carbon Number}

An important choice when simulating the FT reaction system is the choice of maximum carbon number. The work of Khazali (2018) indicates that this truncation point should be chosen based on the contribution of the last point to the distribution. The appropriateness regarding the choice of $N=81$ in the simulations used for model development will be assessed by comparing it with simulations in which $N=121$ is used. This analysis is carried out at a temperature of $220^{\circ} \mathrm{C}$, pressure of 20 bar(a), $\mathrm{H}_{2}$ /CO feed ratio of 2.1 and CO conversion of $10 \%$.

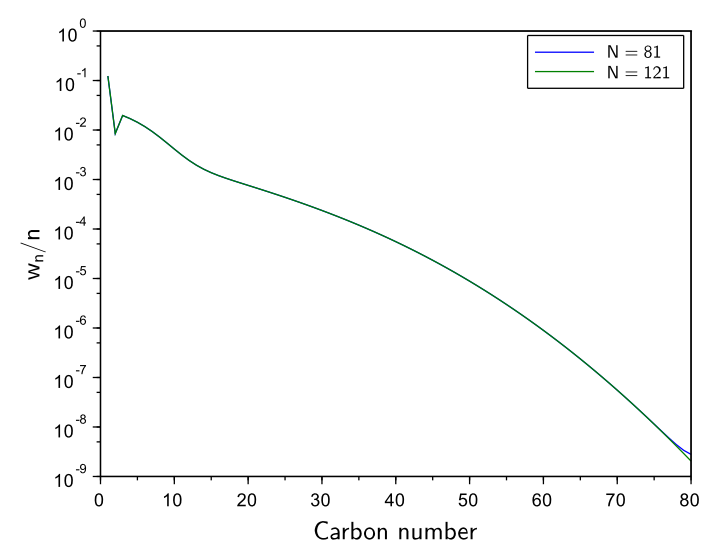

(a) Comparison of the total hydrocarbon distributions

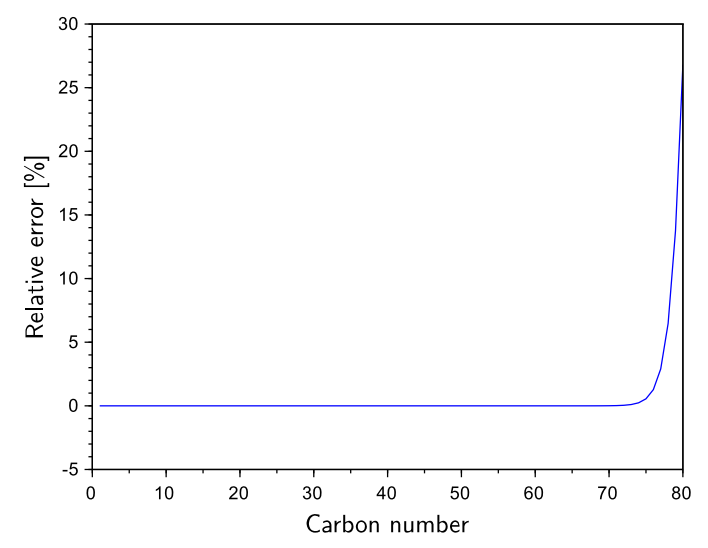

(b) Relative comparison of hydrocarbon quantities

Figure A.2: Simulation of the FT polymerisation behaviour using $N=81$ and $N=121$

From Figure A.2a, the calculated hydrocarbon product distribution when using $N=81$ and $N=121$ are identical, differing only for carbon numbers $C_{76}$ onwards. These two simulations both required a reaction time of $0.882 \mathrm{~kg} . \mathrm{s} / \mathrm{mol} \mathrm{CO}$ to reach the target CO conversion of $10 \%$. This indicates that using $N=81$ will produce the same behaviour as using $N=121$ in terms of the consumption of reactants and the formation of products, while providing savings in terms of computation time. This indicates that the effect of feedback truncation is mitigated and the appropriate estimation of product quantities can effectively be achieved using $N=81$.

In Figure A.2b, the relative error in product quantities using $N=81$ and $N=121$ are compared. It can be seen that again, the two simulations are identical, reiterating the behaviour in Figure A.2a. The increase in relative error from $C_{76}$ onwards arises because $C_{81}$ acts as a barrier in the simulation using $N=81$, while it continues to polymerise when $N=121$. The question then arises is how significant this difference is? The weight fraction of $C_{81}$ is of the order $10^{-6}$. This is well below the limit of detectability (Schwan, 2001). The contribution of $\mathrm{C}_{81}$ to the product distribution is thus negligible. The simulations using $N=81$ and $N=121$ lead to the same CO conversion 
and reaction behaviour, confirming that the effect of feedback truncation has been mitigated in simulating the reaction behaviour. As such, $N=81$ can be used as the truncation point which allows for effective estimation of product quantities while mitigating the impact of feedback on the distribution.

\section{A.3 Equilibrium Constants as a Function of Temperature}

To aid in understanding the behaviour of the FT product distribution i.e. the high methane yield, low ethene yield and the change from mainly 1-olefins at low carbon number to mainly n-paraffins at high carbon number, it is necessary to study the equilibrium constants of reaction $1,2,3, N+2$, $\mathrm{N}+3$ (reaction step 5) and $2 \mathrm{~N}+1$ (reaction step 6) as a function of temperature. This is shown in Figure A.3, calculated using Equation 4.3. Only these reactions are studied because the equilibrium constant of reactions $4-\mathrm{N}+1$ (reaction step 4) and $\mathrm{N}+4-2 \mathrm{~N}$ (reaction step 5) are all equal to unity. This arises because of the linear variation of the ideal gas Gibbs free energy with carbon number. This provides insight into the equilibrium control of the FT product distribution and the cause of the observed changes in the FT product distribution with changing reaction temperature.

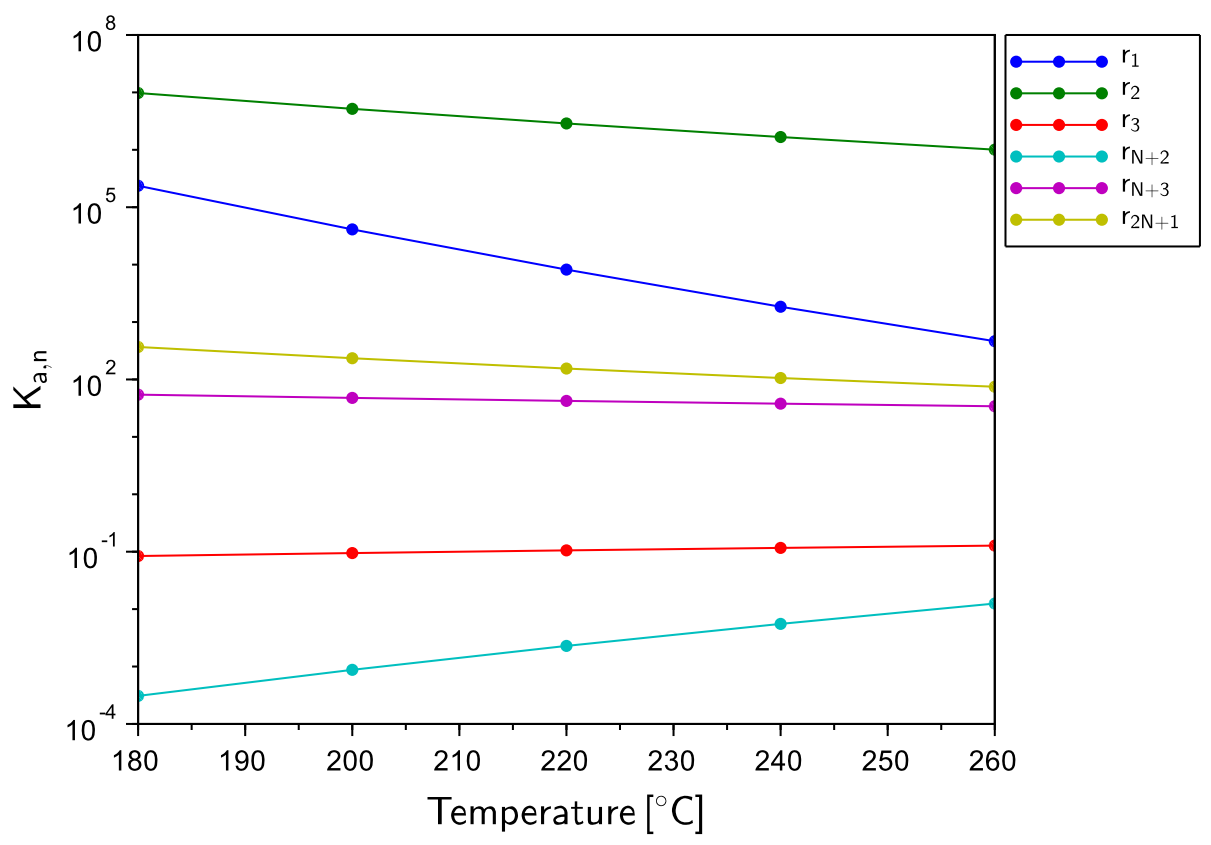

Figure A.3: Equilibrium constants as function of temperature

From Figure A.3, reaction $1,2, N+2$ and $2 \mathrm{~N}+1$ are most strongly affected by the change in reaction temperature. The equilibrium constants $\mathrm{K}_{\mathrm{a}, 1}, \mathrm{~K}_{\mathrm{a}, 2}$ and $\mathrm{K}_{\mathrm{a}, 2 \mathrm{~N}+1}$ all decrease with temperature. This 
indicates that reaction 1, 2 and $2 \mathrm{~N}+1$ are all exothermic. Both reaction 1 and 2 are hydrogenation reactions. As such, this is consistent with these reactions being the drive behind the heat release in the FT reaction system (Förtsch et al., 2015). The equilibrium constant of reaction N+2 increases with temperature. This indicates that $\mathrm{CH}_{2}$ coupling, which leads to the formation of ethene, is endothermic. This is consistent with ethene formation becoming more favourable with increasing temperature (Subiranas, 2009).

The equilibrium constant $\mathrm{K}_{\mathrm{a}, 3}$ for the formation of ethane is an order of magnitude lower than the equilibrium constant for the formation of n-paraffins of carbon number $C_{3}$ and greater. Thus, $\mathrm{n}$-paraffin chain growth is favoured, leading to an $\mathrm{n}$-paraffin product distribution favouring products of higher carbon number. The equilibrium constant $\mathrm{K}_{\mathrm{a}, \mathrm{N}+3}$ for the formation of propene is between three and four orders of magnitude larger than the equilibrium constant $\mathrm{K}_{\mathrm{a}, \mathrm{N}+2}$ for the formation of ethene over the temperature range in Figure A.3 and an order of magnitude larger than the equilibrium constant for the formation of 1-olefins of carbon number $C_{4}$ and greater. This results in propene being the most thermodynamically favoured 1-olefin under FT reaction conditions and a 1-olefin product distribution consisting mainly of products of low carbon number.

It is noted from Figure A.3, that the equilibrium constants of reaction 3 and $\mathrm{N}+3$ are weakly dependent on temperature in comparison to the other reactions. This indicates that the increase in 1-olefin formation and hence the corresponding decrease in $n$-paraffin formation arises because of the decrease in $\mathrm{K}_{\mathrm{a}, 2}$ and increase in $\mathrm{K}_{\mathrm{a}, \mathrm{N}+2}$ with increasing temperature. 


\section{Appendix B}

\section{Sensitivity Calculations}

The purpose of this Appendix is to check the accuracy of the sensitivity functions of the product selectivity as a function of $\mathrm{CO}$ conversion using the first order finite difference approximation with the step size in Equation 5.7. To do this, the calculation is repeated using a second order finite difference approximation, Equation B.1, to see that the same profiles are produced.

$$
\frac{\partial \ln \left(S_{i}\right)}{\partial \ln \left(k_{j}\right)} \approx \frac{k_{j}}{S_{i}\left(k_{j}\right)} \frac{S_{i}\left(k_{j}+\Delta k_{j}\right)-S_{i}\left(k_{j}-\Delta k_{j}\right)}{\Delta k_{j}}
$$

The step size used is the same as in the first order calculation in Equation 5.7. It is acknowledged that the step size used in both the first and second order calculations are not optimal to minimise the total error in the calculation of the derivatives. However, using the optimal value of the first and second order step size lead to the calculation procedure being more erratic and unstable. The value of the step size used ensures that the accuracy of the sensitivity function of all product selectivities are of the same order of magnitude since selectivity is a fractional quantity. This requires a total of thirteen model evaluations and is significantly more computationally expensive than the first order approach. The sensitivity function of the $C_{5+}$ is calculated using Equation 5.8 .

In Figure B.1, the choice of step size in Equation 5.7 is indeed appropriate. This is confirmed by the second order approximation reproducing the curves calculated using the first order approximation. The results of the sensitivity analysis and the interpretation thereof are thus an accurate reflection of the relationship between the model input and model output. 


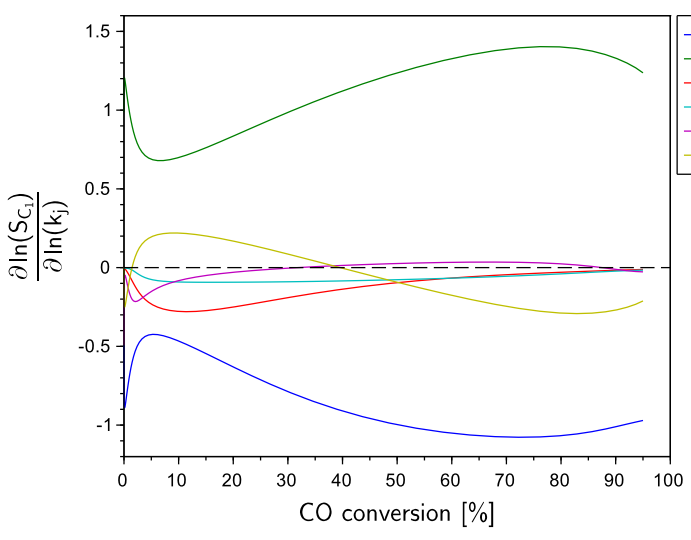

(a) Sensitivity function of $C_{1}$

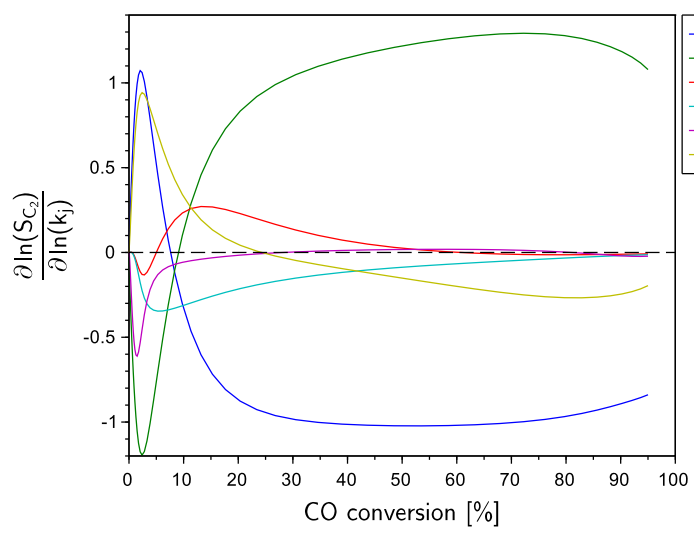

(c) Sensitivity function for $C_{2}$

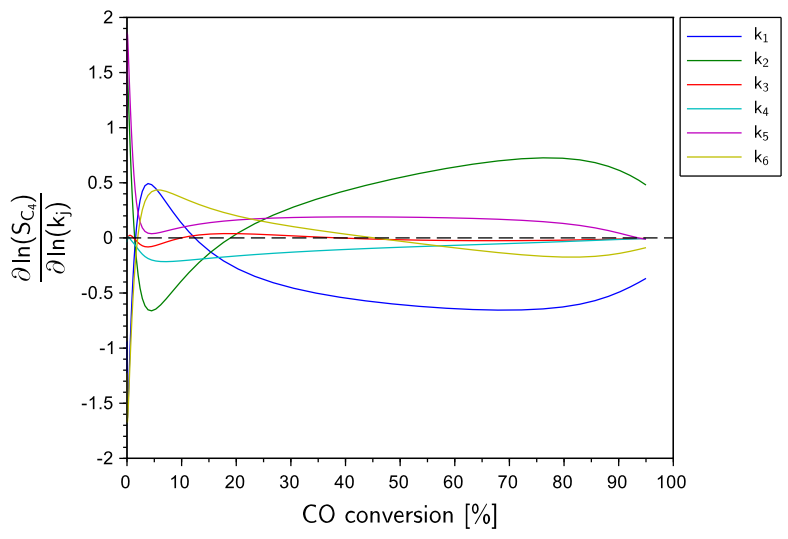

(e) Sensitivity function for $\mathrm{C}_{4}$

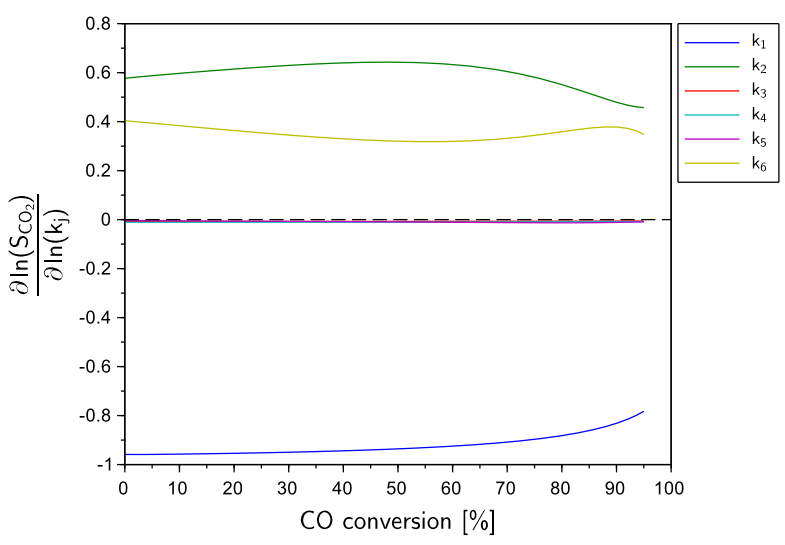

(b) Sensitivity function for $\mathrm{CO}_{2}$

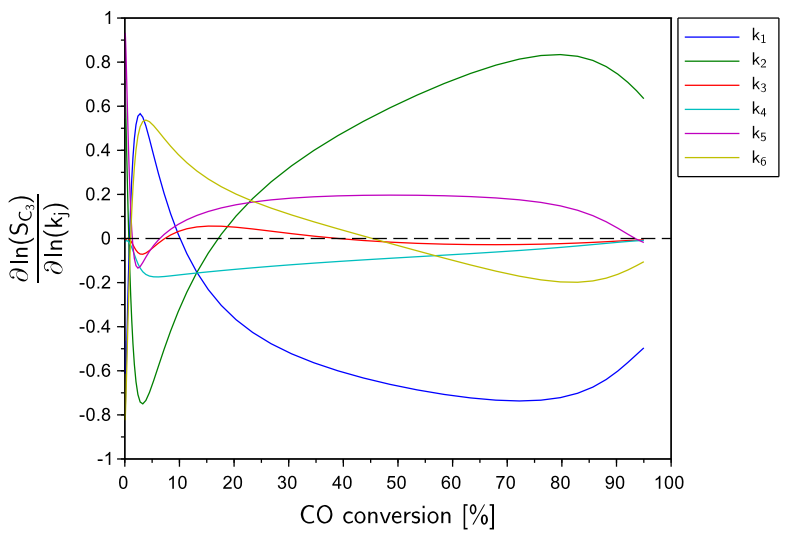

(d) Sensitivity function for $\mathrm{C}_{3}$

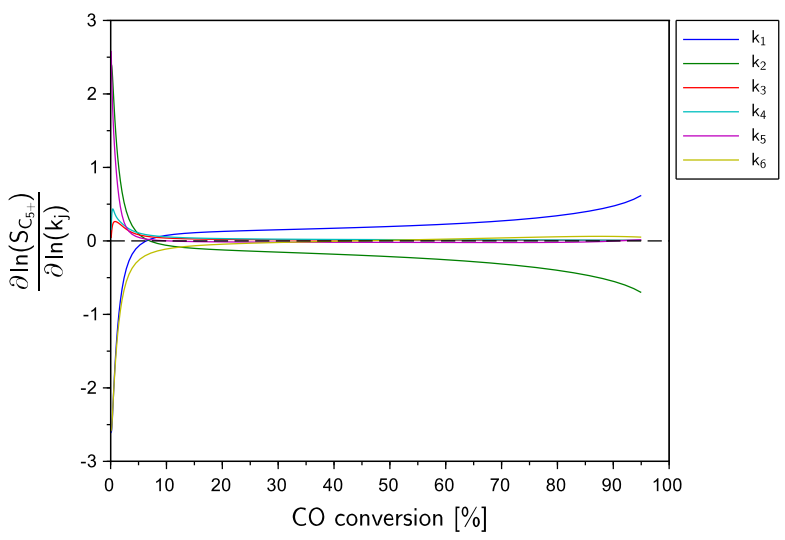

(f) Sensitivity function for $\mathrm{C}_{5+}$

Figure B.1: Sensitivity functions for the $\mathrm{C}_{1}, \mathrm{C}_{2}, \mathrm{C}_{3}, \mathrm{C}_{4}, \mathrm{C}_{5+}$ and $\mathrm{CO}_{2}$ calculated using the second order finite difference approximation. 


\section{Appendix C}

\section{The Need for an Equation of State}

When modelling the effect of VLE on the kinetic behaviour of FT synthesis, an equation of state (EOS), together with an appropriate mixing rule, should be the preferred choice (Masuku et al., 2012a). This is since the reaction mixture consists of molecules of varying size, leading to deviations from ideal behaviour. Raoult's law is thus no longer applicable, especially since its extrapolation to predict the behaviour of heavy hydrocarbons is not guaranteed to be valid. The work of Ahón et al. (2005); Mthombeni (2009); Visconti (2014) and the n-paraffin hydrocracking work of Le Grange (2009) all use the Peng-Robinson (PR)-EOS to model VLE. The appropriateness of this choice can be illustrated by comparing the prediction assuming ideal phases to that of the PR-EOS. This can be analysed using the selectivity to $\mathrm{C}_{1}, \mathrm{C}_{2}, \mathrm{C}_{3}, \mathrm{C}_{4}, \mathrm{C}_{5+}$ and $\mathrm{CO}_{2}$ as a function of $\mathrm{CO}$ conversion at $220^{\circ} \mathrm{C}, 20 \mathrm{bar}(\mathrm{a})$ and $\mathrm{H}_{2} / \mathrm{CO}$ feed ratio of 2.1 . The predicted vapour fraction as a function of $\mathrm{CO}$ conversion using these two approaches will also be compared.

The difference between the two approaches arises through the estimated separation factor. The ideal phases approach uses Equation 7.8. The PR-EOS includes a mixing rule and refines the estimate from Equation 7.8 to yield Equation 7.9 once convergence has been achieved through the flash calculation. This added layer of calculation effort should result in the PR-EOS being more predictive and robust. In Figure C.1. the two approaches yield the same selectivity profiles as a function of $\mathrm{CO}$ conversion at the conditions studied. This indicates that either approach can be used to predict the effect that VLE has on the kinetic behaviour of FT synthesis. The profiles in Figure C.1 do, however, emphasise that limited conclusions regarding the effect of VLE on the kinetic behaviour of $F T$ synthesis can be drawn when using the selectivity norms to $C_{1}, C_{2}, C_{3}$, $\mathrm{C}_{4}, \mathrm{C}_{5+}$ and $\mathrm{CO}_{2}$. This highlights why the use of the product grades in Table 7.2 is more effective to illustrate the effect of VLE when using lumped selectivity measures. 


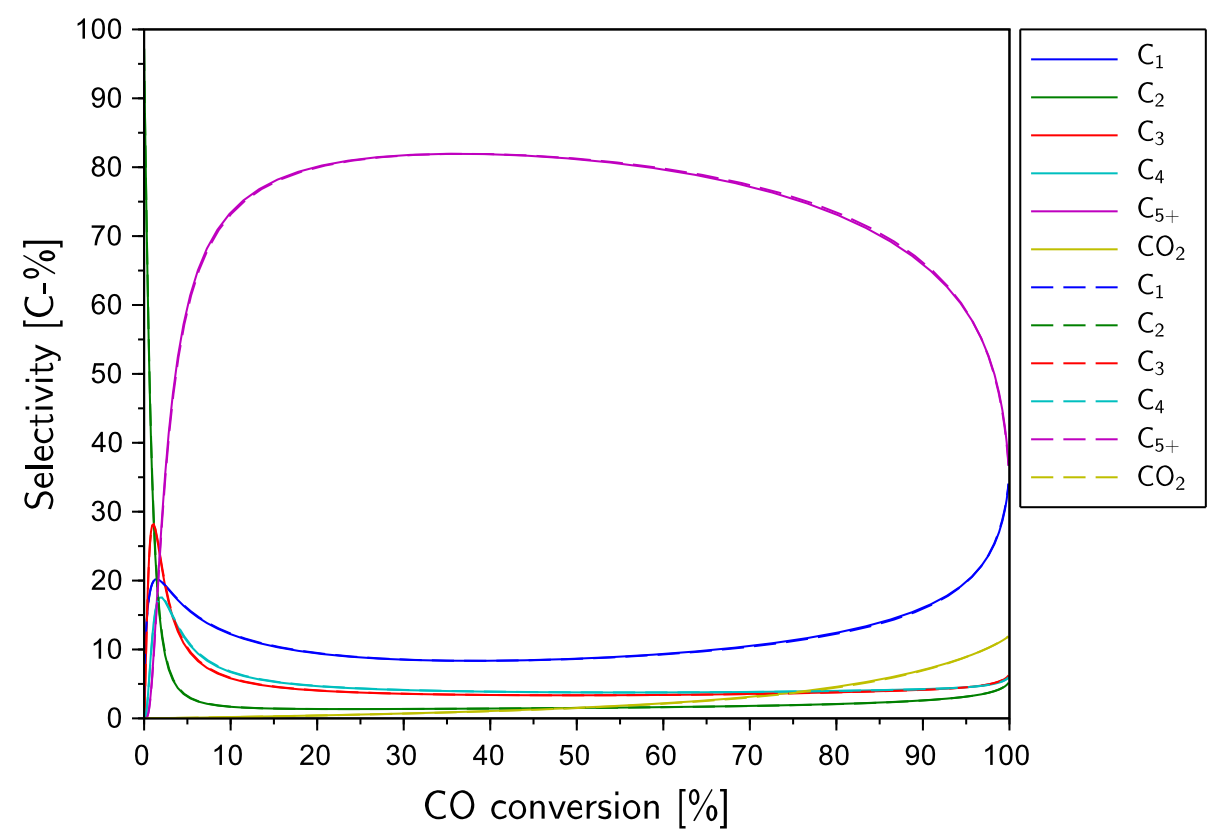

Figure C.1: Selectivity comparison assuming ideal phases and using the PR-EOS at $220^{\circ} \mathrm{C}, 20$ $\operatorname{bar}(\mathrm{a})$ and $\mathrm{H}_{2} / \mathrm{CO}$ feed ratio of 2.1

The difference between the two approaches becomes clear when the predicted vapour fraction profile as a function of $\mathrm{CO}$ conversion is compared in Figure C.2. In Figure C.2a, for CO conversions below 20\%, the ideal phase and PR-EOS approaches estimate similar vapour fractions. This is the $\mathrm{CO}$ conversions regime where the system consists mainly of species in the vapour phase. At the temperature and pressure of the system, the vapour phase fugacity coefficients are close to unity. Thus, the PR-EOS approaches ideal behaviour. The values of the separation factors are thus quite similar. However, above a CO conversion of $20 \%$, considerable differences occur between the two approaches as more liquid forms. For the ideal phases approach, the separation factor is independent of composition and only depends on the temperature and pressure of the system. Thus, throughout the reaction, the value of the separation factor is constant.

The PR-EOS predicts a much higher vapour fraction. This is attributed to the EOS accounting for the changing composition of the mixture in its prediction of the separation factor and being able to more effectively describe the behaviour of species above their critical temperature. This is achieved through the mixing rule which incorporates the likeness of the intermolecular forces of the reaction mixture which consists of mainly non-polar molecules. Consequently, the prediction of the separation factors is more conservative leading to the higher vapour fraction over the simulated CO conversion range in Figure C.2a. The use of an EOS should thus be preferred to describe the 
behaviour of the reaction mixture.

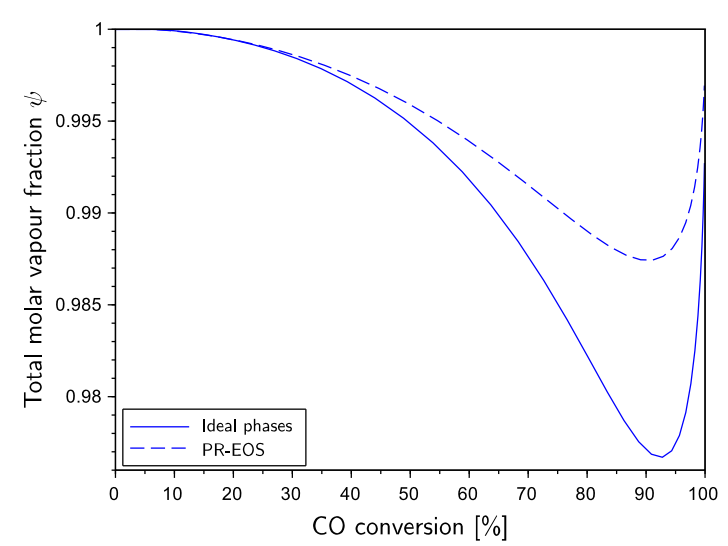

(a) Total molar vapour fraction

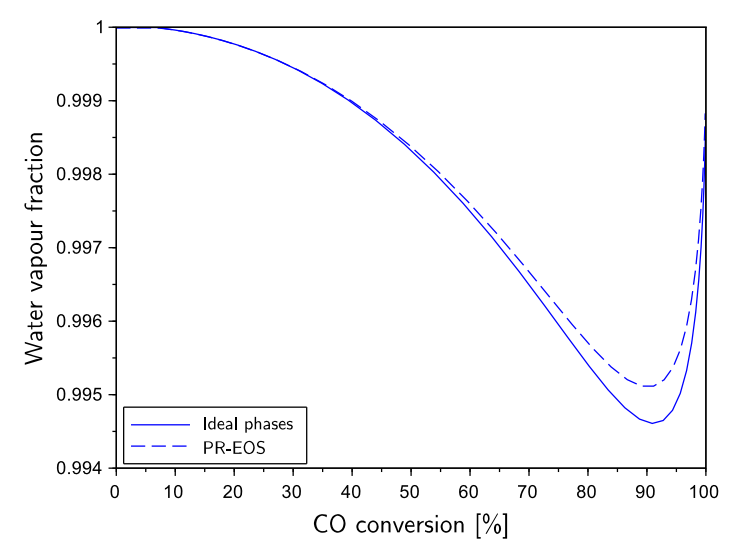

(b) Water molar vapour fraction

Figure C.2: Vapour fraction comparison assuming ideal phases and using the PR-EOS at $220^{\circ} \mathrm{C}$, $20 \operatorname{bar}(\mathrm{a})$ and $\mathrm{H}_{2} / \mathrm{CO}$ feed ratio of 2.1

It was also found that at these conditions, $\mathrm{H}_{2}, \mathrm{CO}$ and hydrocarbon species of carbon number less than $C_{10}$ were completely in the vapour phase. Similar findings were noted by Visconti (2014), although they reported that species of carbon number less than $C_{20}$ were entirely vapour. This is attributed to the temperature of $230^{\circ} \mathrm{C}$ in their simulation and the lumping of products of carbon number $\mathrm{C}_{31+}$ in their flash calculation. From Figure C.2b, water is found to be almost entirely in the vapour phase by both the ideal phases and PR-EOS approaches. This agrees with the findings of Visconti (2014). This is since at the temperature of the simulations, the saturation pressure of water is above the operating pressure of the simulation (see steam tables in Elliot \& Lira (2012)). Ermolaev et al. (2015) indicated that above $210^{\circ} \mathrm{C}$ that water is mainly in the vapour phase. The presence of water mainly in the vapour phase means that the liquid phase consists mainly of nonpolar species. This further highlights that the PR-EOS is an appropriate choice to model the VLE of the FT reaction system. 